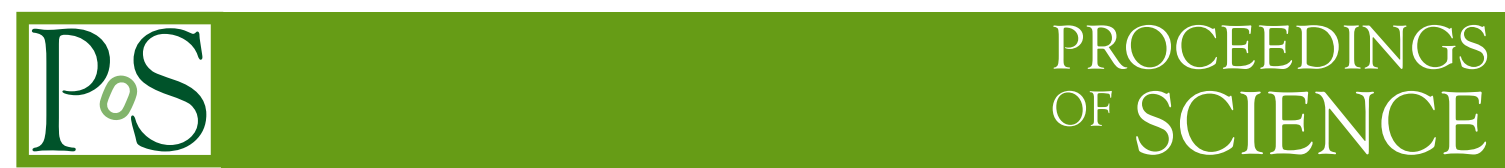

\title{
Topological String Theory on Calabi-Yau threefolds
}

\author{
Albrecht Klemm* \\ UW-Madison Physics Department \\ 1150 University Avenue \\ Madison, WI 53706-1390 \\ E-mail: aklemmephysics.wisc.edu
}

An introduction to the topological A- and B-model on Calabi-Yau threefolds and the techniques to solve them

RTN Winter School on Strings, Supergravity and Gauge Theories

$31 / 1-4 / 22005$

SISSA, Trieste, Italy

\footnotetext{
*Speaker.

$\dagger$ This work is supported in part by DOE grant DE-FG02-95ER40896
} 


\section{Contents}

1. Overview and Motivation 3

2. Semi-classical approximation and super symmetric localization 8

2.1 A simple supersymmetric index 9

3. Supersymmetric nonlinear $\sigma$-models 9

$3.1 \quad N=(1,1)$ nonlinear $\sigma$-model 10

3.2 Compactifications with $N=(2,2)$ world sheet supersymmetry 12

3.3 The $(2,2)$ non-linear $\sigma$-model 12

4. Twisting the $N=(2,2)$ theories and cohomological field theories 14

4.1 Generalities on physical observables 16

4.2 A first look at the metric (in)dependence and topological string theory 18

4.3 A first look at the deformation space 20

4.4 Conformal Field Theory point of view 22

$4.5 t t^{*}$ equations, special geometry and contact terms 27

5. The topological $A$-model 35

5.1 A model without worldsheet gravity 35

5.2 Coupling the A model to worldsheet gravity 38

5.3 Topological gravity 39

5.4 The moduli space of maps

5.5 Idea of localisation and the vertex

5.6 The Gopakumar-Vafa invariants 49

5.7 Relation to Donaldson-Thomas Invariants 51

6. The topological $B$-model 51

6.1 The topological $B$ without worldsheet gravity 52

6.2 First order complex structure deformation 54

6.3 Unobstructedness of the complex deformation space 55

6.4 Kodaira-Spencer gravity as space-time action for the B-model 57

6.5 The periods and infinitesimal deformations of complex structure 58

6.6 Special Kähler geometry 60

6.7 Picard-Fuchs equation from the symmetries of the ambient space 63

6.8 Picard-Fuchs equation from the Dwork-Griffith reduction method 66

6.9 Explicite solutions to the Picard-Fuchs equations 67

6.10 Rational expressions for the threepoint couplings in generic complex structure parameters 70

6.11 Coupling the $B$ model to topological gravity

6.12 The holomorphic anomaly 73 
7. Complex-, Kähler- and Calabi-Yau manifolds. 77

7.1 Complex manifolds

7.2 Kähler manifolds 80

7.3 Characteristic classes of holomorphic vector bundles 83

7.4 Metric Connection and Holonomy 85

7.5 Calabi-Yau manifolds 87

7.6 Bergers List 90

7.7 Examples of Calabi-Yau spaces

\section{Overview and Motivation}

A starting point for studying string theory in a non-trivial space time geometry $M$ is the nonlinear $\sigma$ model. The correlation functions, for simplicity we consider the partition function $Z$ first, are given by a variational integral

$$
Z(M)=\int \frac{\mathscr{D} h}{\text { Vol diff.weyl. }} \mathscr{D} x e^{i S(x, h, M)}
$$

over all embeddings of the world-sheet $\Sigma$ in $M$

$$
x: \Sigma \rightarrow M
$$

and the world-sheet metric $h$. The dependence of such correlation functions on the topology and geometry of $M$, which is treated here as a classical background, might be taken as a first step to describe stringy geometry. It is of direct practical importance as it determines the effective action in $4 \mathrm{~d}$ for string compactifications on $M$. Of particular interest will be the dependence of terms in the low energy effective action on the geometric moduli of $M$. Understanding that this depends on the geometry is a prerequisite for quantizing the latter.

However in the generic case correlation functions like (1.1) are far too complicated to handle. Here we want to study the exceptions. One can be found within super symmetric compactifications of critical string theory. Using diffeomorphism and Weyl invariance, maintained for the critical case in the first quantized version, the dependence on the degrees of freedom of the world-sheet metric $h$ simplifies drastically even in the quantum theory. The world-sheet super symmetry gives rise to nilpotent operators $Q$, which define a theory whose physical operators are cohomology classes w.r.t. $Q$. It is called topological string theory. The reader might wonder how formal the expression (1.1) is. Certainly we have suppressed all fermionic degrees of freedom in $S$. The full actions will be spelled out in Sec. 3. However even if we kill some suspense let us remark that the expression for the integration over $h$, which is just as in the bosonic string in (1.1), is surprisingly accurate for our purpose. It turns out the fermions, which we need to add play merely the rôle that the ghost system plays in the bosonic string.

Physically this reduction to the topological sub sector of the theory can be thought as a semiclassical approximation of (1.1) in which the variational integral is replaced by integral over the 
moduli space $\mathscr{M}$ of the classical solutions $\delta S / \delta x=0$. E.g. for the Polyakov action these are the minimal area maps. The path integral measure collapses to a measure on $\mathscr{M}$, which depends merely on the topological properties of the map (1.2) and on the cohomology classes of the inserted operators. This defines so an intersection theory on $\mathscr{M}$. The intersection numbers are topological invariants of the classical solutions. Examples are the Gromov-Witten invariants, which are symplectic invariants of $M . \sigma$ models with $(2,2)$ world-sheet super symmetry, realized on Calabi-Yau manifolds $M_{6}$, allow for two possibilities to pick $Q$, leading to what is known as the $A$ and the $B$ topological string model[156. Exchanging this choice underlies the mirror duality and which leads to two different ways to solve both models. The $B$-model approach is more effective. Open topological string theory exists as well. Preservation of at least one world-sheet $Q$ operator restricts the boundary conditions on Calabi-Yau three folds with $S U(3)$ holonomy either to special Lagrangian branes for the $A$-model and holomorphic submanifolds for the $B$-model. It had been observed in 1992 that the open topological models are reductions of open string field theory and that this reduction leads to Chern-Simons theories on the branes [148].

The remarkable fact is that in super string theories the restriction to the classical solutions leads to exact calculations of certain low derivative terms in the effective supergravity action in $4 \mathrm{~d}$. This ability to perform exact calculations including non-perturbative effects is typically reflected by nonrenormalization in the effective theory. For example in $N=2$ super symmetric gauge theories the protected terms are the kinetic of the moduli fields $\underline{t}$, which give the exact $\underline{t}$ dependence of the gauge coupling as well as of the masses of the BPS states. Both terms are calculated by genus zero $g=0$ topological string amplitudes. In $N=2$ supergravity theories one obtains from $g>0$ topological string amplitudes the exact moduli dependence of the coupling of the self-dual graviphoton field strength $F_{+}$to the self-dual part of the Ricci curvature $R_{+}$, i.e. the coupling $F_{g}(\underline{t}) R_{+}^{2} F_{+}^{2 g-2}$. In $N=1$ theories one can get the superpotential from disk amplitudes and the gauge kinetic terms from the annulus amplitudes. Reconstruction of these exact terms in the low energy effective action of a field theory by solving the topological string theory in a suitable chosen geometry $M$ is called geometrical engineering.

In general one would like to understand emergence of nearly flat $4 \mathrm{~d}$ space-time $M_{3,1}$ within $M_{9,1}$ dynamically. Often one considers $M_{9,1}=M_{6} \times M_{3,1}$ as ansatz. In generalizations like wrapped geometries [137] or compactifications with RR/NS background fluxes on $M$ [127], which preserve at least $N=1$ supersymmetry one can still use topological string methods to calculate the protected terms. $M_{6}$ being compact leads to traditional compactifications including non-trivial supergravity solutions, as e.g. black hole solutions on $M_{3,1}$. The gauge sector in $M_{3,1}$ can be studied even for non-compact $M_{6}$ if gravity can be consistently decoupled. This is similar to the decoupling of bulk gravity in brane world scenarios with non-compact transversal directions.

The second class of exactly solvable examples are critical string theories [63] [39]. Here the understanding of the infinite symmetries is much more advanced and has lead to the solvability of the string theories with $c \leq 1$ or equivalently $d \leq 2$ dimensions, including the Liouville direction, for the bosonic case. Supersymmetric versions exists as well. For the critical case the quantization of the two dimensional metric degrees of freedom gives rise to the Liouville sector, which augments (1.1) in the quantum theory. The theory consist of ghost-, matter- and Liouville sector and has an nilpotent operator $Q$ with an induced cohomological structure[151], which is strikingly similar to the one in the topological sector of the critical string. The choices of matter are $(p, q)$ minimal 
models for $c<1$ and the free boson for the $c=1$ limiting value[93]. The infinite symmetries which underly the solvability of non-critical string are well understood. An elegant way to summarize the structure is to say that $\log (Z(\underline{t}))$ is the $\tau(\underline{t})$ function associated to a vacuum orbit in an infinite Grassmanian, which is physically described by an infinite $2 \mathrm{~d}$ fermion system.

Major insights in $c \leq 1$ strings have been obtained via the double scaled matrix model [63][39]. The finite $N \times N$ matrix model, for which i.g. several realizations exist, provides a discretization of the string world sheet $\sigma$ in terms of ribbon graphs. A vertex of valence $p$ represents a regular $p$-gon in the dual discretization of $\Sigma$ and it is simplest to fix $p=3$. More importantly the dual $p$-gons of a graph give a discretization of the space of metrics on $\Sigma$ modulo isomorphism. The continuum limit can be understood as an improving approximation of the world-sheet and its metric by graphs with an increasing number $V$ of the vertexes. The key intuition is that for a larger number $V$ of $p$-gons the metric is approximated increasingly accurately by the deficit or surplus angles in gluing the tiles and moreover that the number of graphs which approximate a metric in a given isomorphism class becomes a good measure on the space of metrics. Therefore integrating over metrics can eventually be replaced by counting contributions of the sum of graphs, just as the Feynman graph expansion of the matrix model. The continuum limit requires a regularization procedure in which one takes $N$ to infinity while tuning the coupling(s) of the matrix model to a critical value $g \rightarrow g_{c}$ so that a parameter $t=N\left(g-g_{c}\right)^{\frac{(2-\gamma)}{2}}$ stays finite [39] [152]. The double scaling limit regularizes the total area, whose unregularized value goes like $\langle A\rangle=\langle V\rangle \sim \frac{1}{\left(g-g_{c}\right)}[13]$ as the number of $p$-gons goes to infinity. One can show [13] that a genus $g$ contribution is suppressed with $N^{\chi}$ as $N \rightarrow \infty$ and enhanced with $\left(g-g_{c}\right)^{(2-\gamma) / 2 \chi}$ as $g \rightarrow g_{c}$, where $\chi=2-2 g$. The double scaling definition of $t$ is chosen to counterbalance these effects and to get a finite all genus expansion in $t$.

A qualitative different relation to matrix models is provided by the Kontsevich model [152][99]. It describes the $(2,1)$ pure $2 \mathrm{~d}$ gravity case ${ }^{1}$ by an hermitian matrix model whose ribbon graphs model the cell decomposition of the moduli space $\bar{M}_{g, n}$ of the world-sheet with $n$ descendant operator $\mathscr{O}_{i}$ insertions. The matrix model partition function calculates correlators $\left\langle\mathscr{O}_{1} \ldots \mathscr{O}_{r}\right\rangle$ as topological intersections numbers on $\bar{M}_{g, n}$. The cell decomposition replaces close string insertions by holes and strongly resembles the formalism of open string field theory. The couplings $t_{k}$ of the operators $\mathscr{O}_{k}$ are given in terms of symmetric functions of the hermitian matrix eigenvalues, i.e by the Miura variables $t_{k}=\operatorname{tr} X^{k}$. Results for a given correlator $\left\langle\mathscr{O}_{1} \ldots \mathscr{O}_{r}\right\rangle$ are exact as long as the rank $N$ of the matrix $X$ is large enough to provide enough independent symmetric functions for the $t_{k}$.

Exact calculations in higher dimensional topological strings have been boosted by mirror symmetry [25] and in critical string theory by the double scaled matrix model approach and the Kontsevich type matrix model. The subjects have never been independent as one needs to couple the topological $A$ and $B$ theories to worldsheet gravity to get the $F_{g}$ amplitudes for $g>1$, see [11] for the $B$-model. The solution of pure $2 \mathrm{~d}$ gravity is used explicitly in the calculation of the $A$-model amplitudes by localization [100] together with Hodge integrals[51][95]. A more surprising link between the topological string on the conifold and the $c=1$ string at the selfdual radius [75] has been pointed out in [64].

Two more recent developments motivate to revisit this connection. Dijkgraaf and Vafa observed in 2002 that the exact terms in the effective action of $N=2$ and $N=1$ supersymmetric

\footnotetext{
${ }^{1}$ It has an extension to the coupling of $2 \mathrm{~d}$ gravity to $(1, p)$ matter [99] 154$] 153$.
} 
gauge theories can be calculated also by an hermitian matrix model. Even though this has been explained in the meantime within the supersymmetric field theory framework, it is natural to relate it to topological string calculations by geometrical engineering and in fact it was discovered in this way. This leads to a matrix model descriptions of the topological string on non-compact CalabiYau and the quest for an unified description of the integrable structure behind topological strings in various dimensions[2].

A second motivation comes from the study of open/closed string duality. In the context of noncritical string theory the Kontsevich model has long been considered to be the simplest example of gauge theory/string duality. The gauge theory part describing the open string sector is played by the finite $N$-Kontsevich matrix model, while the closed string part is played by the critical topological string coupled to $(1, p)$ matter. Recent progress in solving the Liouville approach to critical string theory and classifying its boundary conditions revealed that the Kontsevich matrix model emerges as the action on the FZZT brane. This was anticipated from the $B$-model description of open string theory on local Calabi-Yau spaces[2]. It can also be shown by calculating the exact loop-operator in the double scaling limit of the matrix model[110] [78] or by doing a reduction of cubic string field theory[60] on FZZT branes.

An simple example of open/closed string duality in the case of critical topological string theory had been proven by Gopakumar and Vafa in 1999. The closed string side is played by the topological string on the non-compact Calabi-Yau geometry of two complex line bundles over the compact space $\mathbf{P}^{1}$ namely $E^{\prime}=\mathscr{O}(-1) \oplus \mathscr{O}(-1) \rightarrow \mathbf{P}^{1}$. The topological open string geometry is reached from $E^{\prime}$ by contracting the volume $t$ of the $\mathbf{P}^{1}$ and then deforming complex structure of the emerging singular geometry to the smooth cotangent bundle $E=T^{*} S^{3}$ of $S^{3}$. The latter is a Lagrangian submanifold $L$ in $E$ w.r.t to a natural symplectic structure on $E$ and Witten's picture [148] of open topological string relates it to Chern-Simons theory on $S^{3}$. Exact solvability of topological Chern-Simons gauge theory on $S^{3}$ is provided by its relation to the 2d WZW model[150]. The closed topological string on $E^{\prime}$ can be solved exactly by localization [51]. This solvability on both sides provides a luxury, which is not readily available in the analogous situation in the $\mathrm{ADS}_{5} / \mathrm{CFT}$ string/gauge theory correspondence, namely to check explicitly that the partitions functions of gauge- and closed string theory are the same in the large $N$ expansion of Chern-Simons theory when the volume of the $\mathbf{P}^{1}$ is identified with $t=N g_{C S}^{2}$.

Beside the partition function, which is a topological invariant of a three manifold $L$, ChernSimons gauge theory is famous for calculating topological invariants associated to Wilson line expectation values along knots or links inside $L$. What is the topological string question answered by these quantities and what are the new parameters associated to the Wilson line ? A particular answer for the unknot in $S^{3}$ are open string amplitudes ending on a non-compact brane $K$ which meets the $\mathbf{P}^{1}$ of $E^{\prime}$ in an $S^{1}$ [123]. The new parameter is the area of minimal disk ending on the $S^{1}$, which is non-contractible within $K$. The geometry of $E^{\prime}$ and $K$ has a systematic generalization. $E^{\prime}$ contains the algebraic torus $T=\left(\mathbf{C}^{*}\right)^{3}$ as an open subset (one $\mathbf{C}^{*}$ for each line bundle and one for the $\mathbf{P}^{1}$ ). Moreover $\left(\mathbf{C}^{*}\right)^{3}$ acts on $E^{\prime}$ with the natural extension of the multiplicative action of $\left(\mathbf{C}^{*}\right)^{3}$ on itself. Varieties with this property are called toric varieties[59][121] [36][33], here in three complex dimensions. They are characterized by the degeneration of the $T$ action, representable here as linear trivalent graphs embedded in three real dimensions. The vertices represent $\mathbf{C}^{3}$ patches and the graph carries the information about the transition functions. $K$ is characterized by the property 


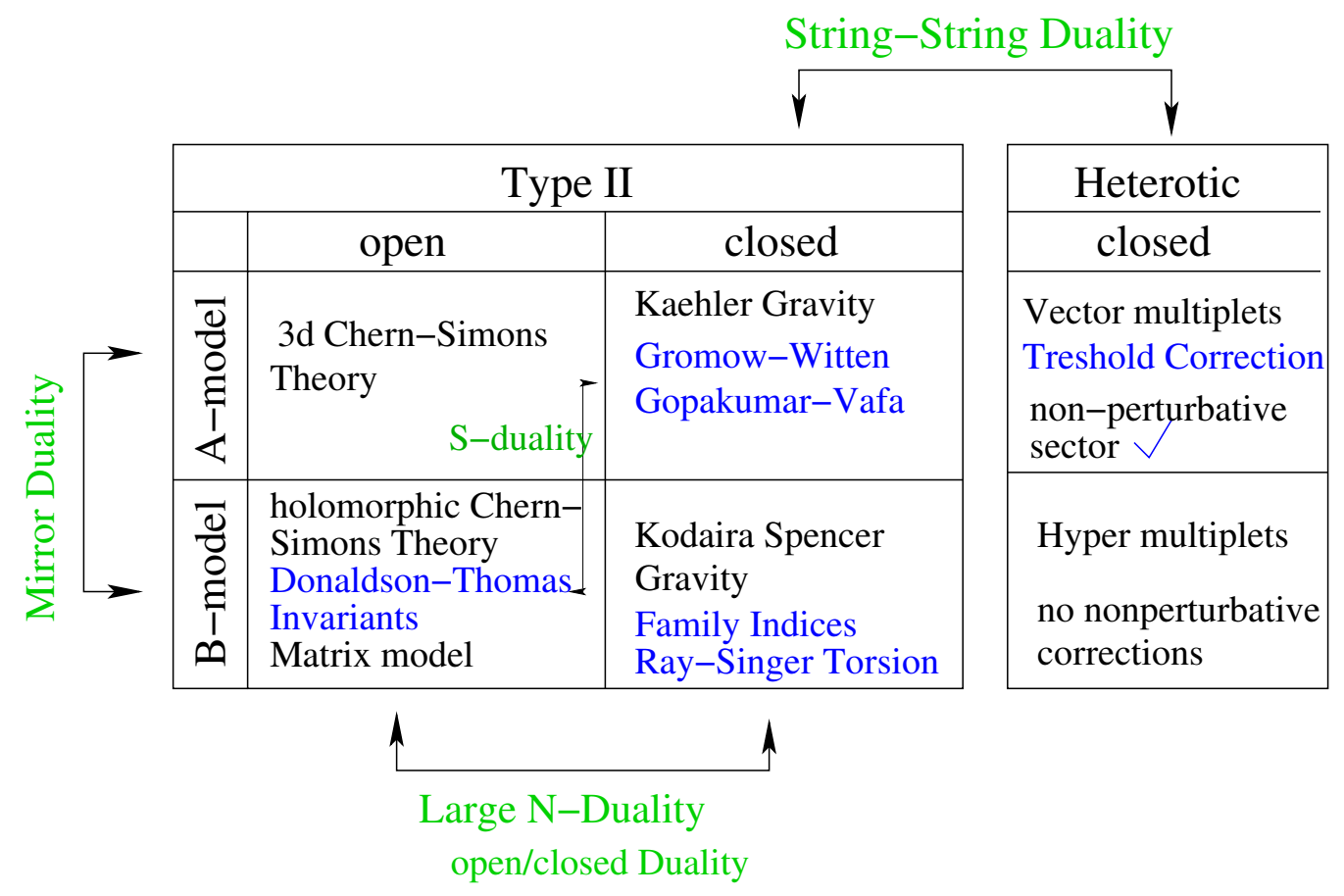

Figure 1: Dualities relevant for the topological string of type II on backgrounds with two and heterotic string in backgrounds with four covaraint constant spinors.

that it is a Lagrangian which is invariant under $\left(\mathbf{C}^{*}\right)^{2} \in T$. Non-compact toric Calabi-Yau manifolds with invariant non-compact special Lagrangian branes are a simple natural class of backgrounds on which all open and closed topological string amplitudes be calculated by localization w.r.t. the torus action. The question how to understand these general amplitudes comes back to ChernSimons gauge theory. The answer is provided by the trivalent topological vertex, which solves the problem for the open topological amplitudes among three stacks of invariant non-compact special Lagrangian branes in a $\mathbf{C}^{3}$ patch, and gluing rules for connecting these amplitudes on a patch to global amplitudes compatible with the global $T$ action. As maybe expected the answer for the vertex is related to the amplitude of a link of three unknots in $S^{3}$.

The exact calculations in the topological sector of string theory have been an indispensable guide to the non-perturbative behavior of critical string theory. Virtually everything known about dualities involving strong coupling regimes is known from the analysis of the topological sub sectors of the corresponding theories. An overview over the dualities in this context is given below

Topological theories come with integrable structures, which reflect their often not immediately apparent symmetries. M-theory gives hints, but the non-perturbative formulation of string theory is illusive. Exploring possible non-perturbative completion of the topological string is a very serious chance in this context. On various aspects of the duality depicted here there have been recently very good lectures. In particular on the connection between matrix models and topological string in [113] and on the connection to Chern-Simons theory and aspects of open/closed duality in [114]. Older physical application of topological string theory using many of the above connections are review in [94] and newer can be found in [117]. Most of the material presented here can be studied 
in more detail in [76]. [146] is an introduction with the virtue of assuming very few prerequisite.

\section{Semi-classical approximation and super symmetric localization}

Let us sketch the reduction of supersymmetric critical string theory to its topological sector. The two dimensional $\sigma$-model action $S(x, h, M)=\int_{\Sigma_{g}} \mathrm{~d}^{2} \sigma \mathscr{L}(x, h, G, B, \ldots)$ depends generically on the metric $G$ of $M$, the NS-two form field $B$ on $M$ and eventually other background fields. A possible attempt to make sense out of (1.1) is to expand the action around the classical solution of the equation of motion $\left.\frac{\delta S}{\delta x}\right|_{x=x_{c l}}=0$

$$
S(x, h, M)=S\left(x_{c l}, h, M\right)+\left.\frac{(\delta x)^{2}}{2} \frac{\delta^{2} S}{\delta^{2} x}\right|_{x=x_{c l}}+\ldots
$$

The quadratic semi-classical approximation in $\delta x$ in (1.1) leads then

$$
\begin{aligned}
Z(M) & =\int \frac{\mathscr{D} h}{\text { Vol diff.weyl. }} \mathscr{D} x e^{i S(x, h, M)} \\
& =\sum_{x_{c l}, h_{c}} e^{i S\left(x_{c l}, h_{c}, M\right)} \int \mathscr{D} \delta x e^{i \frac{(\delta x)^{2}}{2} \frac{\delta^{2} S\left(x_{c l}, h_{c}, M\right)}{\delta^{2} x}} \\
& =\sum_{x_{c l}, h_{c}} e^{i S\left(x_{\left.c l, h_{c}, M\right)}\right)} \operatorname{det}^{-\frac{1}{2}} \frac{\delta^{2} S\left(x_{c l}, h_{c}, M\right)}{\delta^{2} x}
\end{aligned}
$$

Here we have assumed that the determinant can be regularized and we have to consider all classical solutions, which are minimal embeddings of the world-sheet into $M$. It is useful to organize these contributions in a sum over different topological classes of such embeddings as indicated in (2.2). In the closed string case these classes are labeled by the genus of the domain $\Sigma_{g}$ and the cohomology class $H^{2}(M, \mathbf{Z})$ of the image $\left[x\left(\Sigma_{g}\right)\right]$. However depending on the case it might be that there are families of classical solutions of a given topological type parametrized by moduli of the minimal embedding and eventually the complex structure of $h$ called $h_{c}$. In this case one has to integrate over a suitable measure over this moduli space, which is not indicated in the sums in (2.2). Naturally if the semi classical approximation will be good all the configurations "localize" close to extrema of the classical action.

It is a general fact that in supersymmetric extensions of (2.2) there is an exact localization to classical configurations for correlation functions with a suitable fermion zero mode structure. This has its origin simply in the rules of Grassmann integration over the fermionic fields $\Psi_{k}$

$$
\int \Psi_{1} \ldots \Psi_{n} \mathrm{~d} \Psi_{1} \ldots \mathrm{d} \Psi_{n}=1, \quad \int \Psi_{1} \ldots \widehat{\Psi}_{j} \ldots \Psi_{n} \mathrm{~d} \Psi_{1} \ldots \mathrm{d} \Psi_{n}=0
$$

For a field configurations for which the supersymmetric variations do not vanish for all variations of the fermionic fields one can use the supersymmetry transformation to eliminate fermions from the action. By the second identity in (2.3) the fermionic measure will then produce a 0 . Putting the argument around the only contributing field configurations are the ones for which the fermionic variations are stationary, but these are the classical configurations as we will see. 


\subsection{A simple supersymmetric index}

This mechanism is independent of the dimension and can be demonstrated already in the $0 \mathrm{~d}$ case, i.e. for an ordinary integral $Z=\int \mathrm{d} x \mathrm{~d} \Psi_{1} \mathrm{~d} \Psi_{2} e^{-S\left(x, \Psi_{1}, \Psi_{2}\right)}$ over the bosonic variable $x$ and Grassmann variables $\Psi_{1}$ and $\Psi_{2}$. The action

$$
S\left(x, \Psi_{1}, \Psi_{2}\right)=\frac{1}{2}(\partial h)^{2}-\partial^{2} h \Psi_{1} \Psi_{2},
$$

where $h(x)$ is an arbitrary function of $x$. One checks easily that action $\delta S=0$ and measure $\delta\left(\mathrm{d} x \mathrm{~d} \Psi_{1} \mathrm{~d} \Psi_{2}\right)=0$ are invariant under the following supersymmetric transformations

$$
\begin{aligned}
\delta x & =\varepsilon^{1} \Psi_{1}+\varepsilon^{2} \Psi_{2} \\
\delta \Psi_{1} & =\varepsilon^{2} \partial h \\
\delta \Psi_{2} & =-\varepsilon^{1} \partial h .
\end{aligned}
$$

Away from the fixed points of the fermionic transformations, i.e. for $\partial h \neq 0$, we can set $\varepsilon^{1}=\varepsilon^{2}=$ $-\frac{\Psi_{1}}{\partial h}$ and use the supersymmetry transformation to eliminate the first fermion, i.e. with $\hat{x}=x+\delta x$ and $\hat{\Psi}_{i}=\Psi_{i}+\delta \Psi_{i}, i=1,2$ one gets $S\left(\hat{x}, 0, \hat{\Psi}_{2}\right)=S\left(x, \Psi_{1}, \Psi_{2}\right)$. So in the hatted variables there is no $\hat{\Psi}_{1}$ to "soak up" the dî integration and the integral vanishes. To be more explicit we transform the integration measure also to the hatted variables. Since the transformation is singular we consider a nearby transformation $\varepsilon^{2}=(\alpha(x)-1) \frac{\Psi_{1}}{\partial h}, \varepsilon^{1}=-\frac{\Psi_{1}}{\partial h}$ and send $\alpha \rightarrow 0$ after transforming the integral. Note that $\int \Psi \mathrm{d} \Psi=1$ is invariant under $\Psi \rightarrow \hat{\Psi}=\alpha(x) \Psi$, therefore $\mathrm{d} \hat{\Psi}=\frac{1}{\alpha} \mathrm{d} \Psi$. In the transformed integral one finds beside terms which go to 0 with $\alpha$ only a term which is total derivative w.r.t. $\mathrm{d} x$ integral and vanishes at the boundary.

Since the integral gets contributions only from the critical points of $h^{\prime}\left(x_{c}\right)=0$, we can collect the contributions near those points by considering $h(x)=h\left(x_{c}\right)+\frac{\kappa_{c}}{2}\left(x-x_{c}\right)^{2}$, with $\kappa_{c}=h^{\prime \prime}\left(x_{c}\right)$, which yields a Gaussian integration. The partition function

$$
\begin{aligned}
Z= & \frac{1}{2 \pi} \int \mathrm{d} x \mathrm{~d} \Psi_{1} \mathrm{~d} \Psi_{2} e^{-S\left(x, \Psi_{1}, \Psi_{2}\right)}=\sum_{x=x_{c}} \frac{1}{2 \pi} \int \mathrm{d} x \mathrm{~d} \Psi_{1} \mathrm{~d} \Psi_{2} e^{-\frac{1}{2} \kappa_{c}\left(x-x_{c}\right)^{2}+\kappa_{c} \Psi_{1} \Psi_{2}} \\
& =\sum_{x_{c}} \frac{h^{\prime \prime}\left(x_{c}\right)}{\left|h^{\prime \prime}\left(x_{c}\right)\right|} .
\end{aligned}
$$

becomes a primitive version of a supersymmetric index. It counts sum of zeros of $h^{\prime}(x)$ weighted with $+1(-1)$ for positive (negative) slope at $h^{\prime}\left(x_{c}\right)$. If $h^{\prime}(x)$ is continuous a +1 zero of $h^{\prime}(x)$ can only disappear together with a -1 zero under deformations of $h^{\prime}(x)$, which leave the behavior of $h^{\prime}(x)$ for $|x| \rightarrow \infty$ invariant. That means that $Z$ is an invariant under such deformations and can be thought as a topological invariant of $h(x)$.

\section{Supersymmetric nonlinear $\sigma$-models}

Essential features of the $0 d$ topological toy model carry over to super symmetric field theories. In general we search also for field configurations which are fixpoints under some super symmetry transformation. The super symmetry generators become nilpotent operators $Q$ on the Hilbert space of the field theory. The cohomology of $Q$ is a natural structure to extract topological invariants of the classical bosonic configuration space. In more interesting situations indices can occur, which are invariant under some deformations, but are family indices w.r.t. others. Physically the family 
indices can be particular correlation functions. Their dependence on certain geometrical deformation parameters, e.g. of the target space metric, can often be exactly calculated e.g. in an all genus string loop expansion. This is the main physical benefit from topological theories.

The original references for the following are [155, [105] and especially [156]. We have adopted the conventions from the review [76]. The $2 d-\sigma$ model is defined by a map $x: \Sigma \rightarrow M$ from the worldsheet $\Sigma$ to the targetspace $M$. There is a well known dictionary between properties of the worldsheet theory and properties of $M$. In particular to have $(2,2)$ worldsheet supersymmetry $M$ has to be a Kähler manifold [164]. In order to have superconformal invariance $M$ has to be a Calabi-Yau manifold. A Calabi-Yau manifold is Kähler manifold with vanishing first Chern class of its tangent bundle $c_{1}(T M)=0$. This is equivalent to the statement that there exists a hermitean metric $g$ for which the Ricci curvature vanishes $R_{i \bar{j}}=0$. This in turn is equivalent to the statement that the holomomy group of $M$ is contained in $S U(3)$. We call a Calabi-Yau threefold a manifold where the holonomy is the full $S U(3)$ (or a least $S U(2) \times Z_{2}$ ), which implies that there are exactly two covariant constant spinors on $M$. This leads to $N=2$ supergravity theories in $4 \mathrm{~d}$ for the compacification of type II on $M$. Many of the above facts and concepts are reviewed in detail in Sec. 77. We will start the discussion of the symmetries of the actions at the classical level and comment then on the potential anomalies and their cancellation.

\section{1 $N=(1,1)$ nonlinear $\sigma$-model}

Let us first treat the $N=(1,1)$ case. For this case the target space needs to have just a Riemannian metric. We parametrize the map $x: \Sigma \rightarrow M$ by $x^{I}$, where $I \ldots, d$ where $d$ is the real dimension of $M$. The worldsheet is parametrized by $z, \bar{z}$, hence $x$ is given in local coordinates as $x^{I}(z, \bar{z})$ The fields of the $\sigma$ model have the following transformation properties under worldsheet and targetspace reparametrizations. With $K$ and $\bar{K}$ the canonical and anti-canonical bundle of $\Sigma$ and $T M$ the complexified tangentbundle of $M$ one has WS-fermions which transform as $\psi_{+}^{I} \in \Gamma\left(\bar{K}^{\frac{1}{2}} \otimes x^{*}(T M)\right)$ and $\psi_{-}^{I} \in \Gamma\left(K^{\frac{1}{2}} \otimes x^{*}(T M)\right)$, where $\Gamma$ denotes sections of the indicated bundles. The Lagrangian of the non-linear $\sigma$-model is then given by

$$
L=2 t \int_{\Sigma} \mathrm{d}^{2} z\left(\frac{1}{2} g_{I J}(x) \partial_{z} x^{I} \partial_{\bar{z}} x^{J}+\frac{i}{2} g_{I J} \psi_{-}^{I} D_{z} \psi_{-}^{J}+\frac{i}{2} g_{I J} \psi_{+}^{I} D_{\bar{z}} \psi_{+}^{J}+\frac{1}{4} R_{I J K L} \psi_{+}^{I} \psi_{+}^{J} \psi_{-}^{K} \psi_{-}^{L}\right) .
$$

The covariant derivatives $D_{\bar{z}}\left(D_{z}\right)$ are obtained using the pullback of the Levi-Civita connection from $M$ as

$$
D_{\bar{z}} \psi_{+}^{I}=\frac{\partial}{\partial \bar{z}} \psi_{+}^{I}+\frac{\partial x^{J}}{\partial \bar{z}} \Gamma_{J K}^{I} \psi_{+}^{K}
$$

and $R_{I J K L}$ is the Riemann-Tensor of $M$. Here we assumed a flat world-sheet or a local trivialization of $K^{\frac{1}{2}}$, so that no spin connection appears in (3.2). Soon global properties of $K^{\frac{1}{2}}$ and $\bar{K}^{\frac{1}{2}}$ become all important.

With Grassmann valued supersymmetry parameters $\varepsilon_{-} \in \Gamma\left(K^{-\frac{1}{2}}\right)$ and $\varepsilon_{+} \in \Gamma\left(\bar{K}^{-\frac{1}{2}}\right)$ one checks at the classical level the following supersymmetry transformation

$$
\begin{aligned}
\delta x^{I} & =-\varepsilon_{-} \psi_{+}^{I}+\varepsilon_{+} \psi_{-}^{I} \\
\delta \psi_{+}^{I} & =i \varepsilon_{-} \partial x^{I}+\varepsilon_{+} \psi_{-}^{K} \Gamma_{K M}^{I} \psi_{+}^{M} \\
\delta \psi_{-}^{I} & =-i \varepsilon_{+} \partial x^{I}+\varepsilon_{-} \psi_{+}^{K} \Gamma_{K M}^{I} \psi_{-}^{M} .
\end{aligned}
$$


These equations (3.3) are quite similar to (2.5) and we would like to define nilpotent operators from the supersymmetry transformations. The obstruction is that there are no global trivial sections of $K^{\frac{-1}{2}}$ or $\bar{K}^{-\frac{1}{2}}$ unless $g=1$. This means that there no global supersymmetry transformations on the worldsheet unless ${ }^{2} g=1$.

In the case of the worldsheet beeing a torus one can chose globally defined sections $\varepsilon_{-} \in$ $\Gamma\left(K^{-\frac{1}{2}}\right)$ and $\varepsilon_{+} \in \Gamma\left(\bar{K}^{-\frac{1}{2}}\right)$ to obtain globally defined supersymmetry generators $Q_{-}^{2}=0$ and $Q_{+}^{2}=0$ on the Hilbert space $\mathscr{H}$. E.g. we can chose $\varepsilon_{ \pm}$both to be in trivial sections of $K^{-\frac{1}{2}}$ and $\bar{K}^{-\frac{1}{2}}$ respectively. In view of 3.3 we have to chose corresponding trivializations for $\psi_{+}^{I} \in \Gamma\left(\bar{K}^{\frac{1}{2}} \otimes x^{*}(T M)\right)$ and $\psi_{-}^{I} \in \Gamma\left(K^{\frac{1}{2}} \otimes x^{*}(T M)\right)$ and this simply means that the fermions will have periodic boundary conditions on $T^{2}$. These boundary conditions are called twisted boundary conditions. $Q_{-}$and $Q_{+}$ are globaly defined and $Q_{+}|\Psi\rangle=Q_{-}|\Psi\rangle=0$ for $\Psi \in \mathscr{H}$ forces the cohomological states to be in the $E=0$ super symmetric ground state of the Hamiltonian $H=\frac{1}{2}\left\{Q_{+}, Q_{-}\right\}=\frac{1}{2}\left(\mathrm{dd}^{*}+\mathrm{d}^{*} \mathrm{~d}\right)[157]$. This reduces the model to constant maps, i.e. supersymmetric quantum mechanics. The $\sigma$ model cohohomology is equivalent to de Rham cohomology of $M$, much in the same way as we will made explicit in Sec. 5.1 and 6.1. The only non vanishing correlator in the double twisted model is the Witten index, which is easily shown be equal to the Euler number of $M$ [157]. It is simplest written in the Hamiltonian formalism

$$
\chi(M)=\operatorname{Tr}(-1)^{F} q^{H_{+}} \bar{q}^{H_{-}}=\operatorname{Tr}(-1)^{F},
$$

where $F=F_{+}+F_{-}$and $F_{+} / F_{-}$count the left/right moving fermion numbers so that $\left\{(-)^{F_{ \pm}}, Q_{ \pm}\right\}=$ 0 while $\left[(-)^{F_{\mp}}, Q_{ \pm}\right]=0$. Note that $(-1)^{F}=(-1)^{F_{+}+F_{-}}$can be defined even if the individual fermion numbers are anomalous.

A much more interesting situation arises if one choses only $\varepsilon_{+}$to be in a trivial section. The corresponding index is called the elliptic genus ${ }^{3}$

$$
\mathscr{E}(M)=\operatorname{Tr}(-1)^{F_{+}} q^{H_{+}} \bar{q}^{H_{-}}=\operatorname{Tr}(-1)^{F_{+}} q^{H_{-}} .
$$

Here only the left moving states are forced in the left moving groundstate. The trace over the right moving states explores information which goes far beyond cohomological information of $M$. It can be defined for $2 \mathrm{~d}$ supersymmeric field theories and is conformally invariant even if the underlying field theory is not [160]. It requires $(-)^{F_{+}}$not to be anomalous, which is essentially equivalent to $M$ being spin [162]. It carries information, which is robust under certain deformations. In the case of the $\sigma$ model on $M \mathscr{E}(M)$ is the Dirac index of the loop space of $M[158,159]$. This index varies with the volume parameters of $M$, but is independent of the complex structure of $M$ and is the first example of the promised family indices. There are further simple refinements possible, if as below in the $N=(2,2)$ theories $F_{-}$comes from an $U(1)_{L}$ current $F_{-}=\oint J_{L}$. If the latter is not anomalous one can insert $(-1)^{\theta F_{-}}$in the trace in (3.5) and even if the $U(1)_{L}$ is broken to $Z_{K}(3.5)$ with $\exp \left(\frac{i \pi}{k} F_{-}\right)$inserted is still an index. A theme of the lecture is to explore more sophisticated family indices mainly in the $N=(2,2)$ context and even at genus one there are further refinements such as (6.111).

\footnotetext{
${ }^{2}$ The quest for covariant constant spinors is familiar on the target space in order to obtain spacetime supersymmetric compactifications. It requires restricted holonomies, see section 7.6, which is equivalent to the familiar $c_{1}(T M)=0$ condition for $N=2(N=1)$ II (heterotic) compactifications $6 \mathrm{~d}$ internal manifolds.

${ }^{3}$ Unfortunately there are many notations common to distinguish the left- and right moving sectors in this context unbarred/barred for euclidean worldsheets, $R / L,+/-$ and without tilde/with tilde are maybe most often used.
} 


\subsection{Compactifications with $N=(2,2)$ world sheet supersymmetry}

The additional structure that allows to define more general family indices for the $(2,2)$ worldsheet theories are right and left $U(1)_{R / L}$ symmetries, so called $R$-symmetries. Since the nilpotent $Q$ operators are derived from the supersymmetry transformations and since there are no covariant constant spinors for world sheets of genus $g \neq 1$ there will be no well defined supersymmetry operators on general $\Sigma_{g}$ without further modifications. For the topological theory to make sense at all genus $g$ we "change" the transformation properties of the fields, so that the supersymmetry transformation becomes a scalar operator on the world sheet. This modification is implemented by twisting the world sheet Lorentz group either by the vector $U(1)_{V}=U(1)_{L}+U(1)_{R}$ or the axial $U(1)_{A}=U(1)_{L}-U(1)_{R}$ symmetry. To do this we first gauge the $R$-symmetries. Then we combine the $U(1)$ gauge connection with the spin connection to a twisted world sheet spin connection. Contrary to the $U(1)_{V}$ the $U(1)_{A}$ current develops a quantum anomaly proportional to $\int_{\Sigma} x^{*}\left(c_{1}(T M)\right)$. Therefore the $B$ model, which is obtained by twisting with the $U(1)_{A}$ connection, is only well defined on Calabi-Yau manifolds $\left(c_{1}(T M)=0\right)$, while the $A$ model, which is obtained by twisting with the $U(1)_{V}$ connection can be considered on any Kähler manifold.

\subsection{The $(2,2)$ non-linear $\sigma$-model}

Let us now see this mechanism in Kähler case, which has at the classical level a $N=(2,2)$ supersymmetry and hence the necessary $U(1)$ symmetries. The action is given by

$$
S=2 t \int_{\Sigma} \mathrm{d}^{2} z\left(-g_{i \bar{j}} \partial_{\mu} x^{i} \partial^{\mu} x^{\bar{j}}+i g_{\bar{i} i} \psi_{-}^{\bar{i}} D_{z} \psi_{-}^{i}+i g_{\bar{i} i} \psi_{+}^{\bar{i}} D_{\bar{z}} \psi_{+}^{i}+R_{i \bar{i} j \bar{j}} \psi_{+}^{i} \psi_{+}^{\bar{i}} \psi_{-}^{j} \psi_{-}^{\bar{j}}\right)
$$

Here we have split the index $I$ into $i$ and $\bar{i}$ according to the Kähler decomposition. A Kähler metric can locally be written as $g_{i \bar{j}}=\partial_{i} \partial_{\bar{j}} K\left(x^{i}, x^{\bar{l}}\right)$ and its Levi-Civita connection in Kähler geometry is pure in the indices $\Gamma_{j k}^{i}=g^{i j} \partial_{j} g_{k, j}$ as discussed in more detail in Sec. 7.2. On a non-flat Riemann surface $\Sigma$ one has the connection

$$
\begin{aligned}
& D_{\bar{z}} \psi_{+}^{i}=\partial_{\bar{z}} \psi_{+}^{i}+\frac{i}{2} \omega_{\bar{z}} \psi_{+}^{i}+\Gamma_{k l}^{i} \partial_{\bar{z}} x^{k} \psi_{+}^{l} \\
& D_{z} \psi_{-}^{i}=\partial_{z} \psi_{-}^{i}-\frac{i}{2} \omega_{z} \psi_{+}^{i}+\Gamma_{k l}^{i} \partial_{z} x^{k} \psi_{-}^{l},
\end{aligned}
$$

where $\omega_{z}$ and $\omega_{\bar{z}}$ are the components of the spin connection of $\Sigma$.

In superfield formalism can can write $L=2 t \int \mathrm{d} \theta^{4} K\left(\mathbf{X}^{i}, \overline{\mathbf{X}}^{\bar{\imath}}\right)$, where the chiral field $\mathbf{X}^{i}$ has components $x^{i}, \psi_{ \pm}^{i}, F^{i} . F^{i}$ is an auxiliary field that has has no kinetic terms and can be eliminated from the action by its equation of motion $F=\Gamma_{i j}^{i} \psi_{+}^{j} \psi_{-}^{k}$. This offshell superfield formalism is particularly useful when one couples a holomorphic superpotential $W\left(x^{i}\right)$ to the action, which is only possible for non-compact target spaces $M$. This formalism is worked out in detail including the off-shell supersymmetry transformations in [105] and reviewed in [76]. For notational brevity we restrict ourselves to the onshell formalism.

Classically there are now twice as many super symmetries, one set for the holomorphic and one set for the antiholomorphic space time indices. They generated by $\varepsilon_{+} \in \Gamma\left(K^{\frac{1}{2}}\right), \varepsilon_{-} \in \Gamma\left(\bar{K}^{\frac{1}{2}}\right)$ and $\bar{\varepsilon}_{ \pm}$. The latter are sections of the same bundles but have opposite charges under $U(1)_{A}$ and 
$U(1)_{V}$

$$
\begin{aligned}
\delta x^{i} & =-\varepsilon_{-} \psi_{+}^{i}+\varepsilon_{+} \psi_{-}^{i} \\
\delta x^{\bar{i}} & =\bar{\varepsilon}_{-} \psi_{+}^{\bar{i}}-\bar{\varepsilon}_{+} \psi_{-}^{\bar{i}} \\
\delta \psi_{+}^{i} & =2 i \bar{\varepsilon}_{-} \partial_{+} x^{i}+\varepsilon_{+} \psi_{+}^{j} \Gamma_{j m}^{i} \psi_{-}^{m} \\
\delta \psi_{+}^{\bar{i}} & =-2 i \varepsilon_{-} \partial_{+} x^{\bar{i}}+\bar{\varepsilon}_{+} \psi_{-}^{\bar{j}} \Gamma_{\bar{j} \bar{m}}^{\bar{i}} \psi_{+}^{\bar{m}} \\
\delta \psi_{-}^{i} & =-2 i \bar{\varepsilon}_{+} \partial_{-} x^{i}+\varepsilon_{-} \psi_{+}^{j} \Gamma_{j m}^{i} \psi_{-}^{m} \\
\delta \psi_{-}^{\bar{i}} & =2 i \varepsilon_{+} \partial_{-} x^{\bar{i}}+\bar{\varepsilon}_{-} \psi_{-}^{\bar{j}} \Gamma_{\bar{j} \bar{m}}^{\bar{i}} \psi_{+}^{\bar{m}} .
\end{aligned}
$$

The relation between the existence of the two has been discussed first by [164]. Decomposition of the exterior derivative on Kähler manifolds into a holomorphic and antiholomorphic derivative $\mathrm{d}=\bar{\partial}+\partial$, which gives rise to Hodge decomposition of cohomology groups into $H^{p, q}(M)$. The fields $x^{i}, x^{\bar{l}}, \psi_{ \pm}^{i}$ and $\psi_{ \pm}^{\bar{i}}$ transform as before under WS transformations. W.r.t. the spacetime transformations one has now simply a splitting of $T M_{\mathbf{C}}$ into $T^{1,0} M \oplus T^{0,1} M$ with $i$ referring to $T^{1,0} M$ and $\bar{\imath}$ referring to $T^{0,1} M$, so e.g. $\psi_{+}^{i} \in \Gamma\left(\bar{K}^{\frac{1}{2}} \otimes x^{*}\left(T^{1,0} M\right)\right)$ e.t.c. All transformation properties are summarized in table 1 .

The action of the $U(1)_{V}$ and $U(1)_{A}$ are conveniently formulated in superfield formalism, i.e. expand any field in Grassmann valued $\theta^{+}, \theta^{-}, \bar{\theta}^{+}, \bar{\theta}^{-}$complex fermionic spinor coordinates on which complex conjugation is given by $\left(\theta^{ \pm}\right)^{*}=\bar{\theta}^{ \pm}$. The WS Lorentz transformation acts on $t=x^{0}$ and $s=x^{1}$ (with $(1,1)$ signature) and on spinors as

$$
\begin{aligned}
\left(\begin{array}{c}
x^{0} \\
x^{1}
\end{array}\right) & \rightarrow\left(\begin{array}{c}
\cosh \gamma \sinh \gamma \\
\sinh \gamma \cosh \gamma
\end{array}\right)\left(\begin{array}{c}
x^{0} \\
x^{1}
\end{array}\right) \\
\theta^{ \pm} & \rightarrow e^{ \pm \frac{\gamma}{2}} \theta^{ \pm} \\
\bar{\theta}^{ \pm} & \rightarrow e^{ \pm \frac{\gamma}{2}} \overline{\boldsymbol{\theta}}^{ \pm}
\end{aligned}
$$

Since the fermionic variables anticommute w.r.t. to each other the Taylor expansion in them contains only $2^{4}$ terms

$\Phi\left(x, \theta^{ \pm}, \bar{\theta}^{ \pm}\right)=x(t, s)+\theta^{+} \psi_{+}(t, s)+\theta^{-} \psi_{-}(t, s)+\bar{\theta}^{+} \bar{\psi}_{+}(t, s)+\bar{\theta}^{-} \bar{\psi}_{-}(t, s)+\theta^{+} \theta^{-} A_{+-} s, t+\ldots$

In this sense one can think of superspace as a thin space in the fermionic directions, which contains no second order derivative information in a given fermionic direction. The relation to calculus with differential forms is very obvious. The action of the vector $U(1)_{V}$ and axial $U(1)_{A}$ symmetries on all component fields is induced from

$$
\begin{aligned}
& e^{i \alpha F_{V}}: \Phi\left(x, \theta^{ \pm}, \bar{\theta}^{ \pm}\right) \mapsto e^{i \alpha q_{V}} \Phi\left(x, e^{-i \alpha} \theta^{ \pm}, e^{i \alpha} \bar{\theta}^{ \pm}\right) \\
& e^{i \beta F_{A}}: \Phi\left(x, \theta^{ \pm}, \bar{\theta}^{ \pm}\right) \mapsto e^{i \beta q_{A}} \Phi\left(x, e^{\mp i \beta} \theta^{ \pm}, e^{ \pm i \beta} \overline{\boldsymbol{\theta}}^{ \pm}\right) .
\end{aligned}
$$

Let us denote now the four supersymmetry operators corresponding to $\varepsilon^{ \pm}$and $\bar{\varepsilon}^{ \pm}$transformations $Q_{\mp}$ and $\bar{Q}_{\mp}$ respectively. A general supersymmetry transformation is then generated by the operator

$$
\hat{\delta}=i \varepsilon_{+} Q_{-}-i \varepsilon_{-} Q_{+}-i \bar{\varepsilon}_{-} \bar{Q}_{-}+i \bar{\varepsilon}_{+} \bar{Q}_{+}
$$


where $\left(Q^{ \pm}\right)^{\dagger}=\bar{Q}_{ \pm}$and $\hat{\delta}^{\dagger}=-\hat{\delta}$.

More generally for any infinitesimal field transformation $\delta_{Q} \phi$ we will denote the infinitesimal transformation on the field operator $\delta \mathscr{O}_{\phi}$ by $\delta_{Q} \mathscr{O}_{\phi}=\left[Q, \mathscr{O}_{\phi}\right]_{ \pm}$, where $Q$ is the corresponding generating operator. Let $M$ be the generator of two dimensional Lorentz rotations $S O(1,1)$. It is convenient to make the Wick rotation $x^{0}=-i x^{2}$ and we call $M_{E}=i M$ the generator of the compact Euclidean rotation group $U(1)_{E}$. Beside the supersymmetry generators one has on the WS $H$ the generator of (euclidean) time translations, $P$ generator of translations. Furthermore there are the $R$-charge operators associated to the $U(1)_{V}$ and $U(1)_{A}$ currents called $F_{V}$ and $F_{A}$. These generators fulfill the algebra

$$
\begin{aligned}
Q_{+}^{2}= & Q_{-}^{2}=\bar{Q}_{+}^{2}=\bar{Q}_{-}^{2}=0, \\
\left\{Q_{ \pm}, \bar{Q}_{ \pm}\right\} & =H \pm P, \quad\left\{\bar{Q}_{+}, \bar{Q}_{-}\right\}=\left\{Q_{+}, Q_{-}\right\}=\left\{Q_{-}, \bar{Q}_{+}\right\}=\left\{Q_{+}, \bar{Q}_{-}\right\}=0, \\
{\left[M_{E}, Q_{\mp}\right] } & =\mp Q_{ \pm}, \quad\left[M_{E}, \bar{Q}_{ \pm}\right]=\mp \bar{Q}_{ \pm}, \\
{\left[F_{V}, Q_{ \pm}\right] } & =-Q_{ \pm}, \quad\left[F_{V}, \bar{Q}_{ \pm}\right]=\bar{Q}_{ \pm}, \\
{\left[F_{A}, Q_{ \pm}\right] } & =\mp Q_{ \pm}, \quad\left[F_{V}, \bar{Q}_{ \pm}\right]= \pm \bar{Q}_{ \pm},
\end{aligned}
$$

It becomes soon important that $Q_{ \pm}$and $\bar{Q}_{ \pm}$have opposite charges under the $R$ symmetry groups. As already stated $F_{A}$ is present at the quantum level only for Calabi-Yau manifolds, the conformal case, while $F_{V}$ is generically present. See [105] for a further discussion of this algebra.

\section{Twisting the $N=(2,2)$ theories and cohomological fi eld theories}

Twisting amounts to a modification of the Euclidean rotation group $U(1)_{E}$ by a generator of the global $U(1) R$-symmetry groups and define the new generator of the Euclidean rotation group $U(1)_{E^{\prime}}$ as $M_{E}^{\prime}=M_{E}+R$.

Another way of saying this is that one gauges the $U(1)-\mathrm{R}$ symmetry group and adds the corresponding gauge connection $A_{\mu}^{R}$ to the spin connection, so that the transformation property of the spinor fields depend now on their $R$ charge. Denote the gauge current, which corresponds to the gauge variations $\delta A_{\mu}^{R}$ by $J_{\mu}^{R}$. It will modify the energy momentum tensor to

$$
\hat{T}_{\mu v}=T_{\mu v}+\frac{1}{4}\left(\varepsilon_{\mu}^{\lambda} \partial_{\lambda} J_{v}^{R}+\varepsilon_{v}^{\lambda} \partial_{\lambda} J_{\mu}^{R}\right)
$$

In the action of the gauged theory there is a coupling

$$
\Delta S=\int_{\Sigma} J^{\mu} \omega_{\mu}=\frac{1}{2} \int_{\Sigma} J \bar{\omega}+\bar{J} \omega=\frac{1}{2} \int_{\Sigma} R \phi+\text { total der. },
$$

to the spin connection $\omega$. In the second equality we bosonized the $U(1)_{R}$ current $\partial \phi=J$ and integrated partially. Contact terms of operators with the this expression will play a rôle determining properties of the correlation functions.

Because of different signs under which the different chiral components of the spinors transform the $U(1)_{A}$, the axial current develops an anomaly proportional to the Dirac index, which is related by the Atiyah-Singer index theorem to the index, which is calculate with the Hirzebruch-Riemann Roch theorem in Sec. (7.3) to be $\int_{\Sigma_{g}} x^{*}\left(c_{1}(T M)\right)$. Path integral methods for deriving the anomaly 


\begin{tabular}{|c|c|c|c|}
\hline & Section before wisting & Section (+) twist & Section (-) twist \\
\hline$x$ & $x^{*}(T M)$ & $x^{*}(T M)$ & $x^{*}(T M)$ \\
$\psi_{-}^{i}$ & $x^{*}\left(T^{1,0}\right) \otimes K^{\frac{1}{2}}$ & $x^{*}\left(T^{1,0}\right)$ & $x^{*}\left(T^{1,0}\right) \otimes K$ \\
$\bar{\psi}_{-}^{\bar{l}}$ & $x^{*}\left(T^{0,1}\right) \otimes K^{\frac{1}{2}}$ & $x^{*}\left(T^{0,1}\right) \otimes K$ & $x^{*}\left(T^{0,1}\right)$ \\
$\psi_{+}^{i}$ & $x^{*}\left(T^{1,0}\right) \otimes \bar{K}^{\frac{1}{2}}$ & $x^{*}\left(T^{1,0}\right)$ & $x^{*}\left(T^{1,0}\right) \otimes \bar{K}$ \\
$\bar{\psi}_{+}^{\bar{l}}$ & $x^{*}\left(T^{0,1}\right) \otimes \bar{K}^{\frac{1}{2}}$ & $x^{*}\left(T^{0,1}\right) \otimes \bar{K}$ & $x^{*}\left(T^{0,1}\right)$ \\
\hline
\end{tabular}

Table 1: Space time transformation of the non linear $\sigma$-model fields after + and - twist. Classically and in non-anomalous theories one can chose the twisting on the left movers $\psi_{-}^{i}, \psi_{-}^{\bar{l}}$ and the right movers $\psi_{+}^{i}, \psi_{+}^{\bar{l}}$ independently.

are reviewed in [57]. The $U(1)_{V}$ vector current is always non anomalous at quantum level. For a discussion of the $U(1)_{A}$ anomaly in the linear $\sigma$-model context see [162].

The most desired effect of this twisting is that some of the $Q_{ \pm}$and $\bar{Q}_{\mp}$ can be made to transform as scalars under $U(1)_{E^{\prime}}$. These "scalar" operators are then globally defined on worldsheets of arbitrary genus and can be used to define a cohomological theory on an arbitrary Riemann surface. The term twisting is familiar in the orbifold context, where it means to modify the boundary conditions of a field along cycles of the worldsheet by an element $g$ of a global symmetry group $G$, e.g. for the torus with a $A$ cycle of length $2 \pi$ a field is periodically identified by $\phi(x+2 \pi)=g \phi(x)$. The analogy is appropriate since also in the above case we change the boundary conditions of some fermionic fields to become periodic. We encountered such twisting already in the discussion of Witten index and the elliptic genus. The twisting changes the WS transformation properties of the fields. The table below records this for the so called + and the - twist.

In the $(2,2)$ theory we have two fundamentally different possibilities to twist

$$
\begin{array}{ll}
\mathrm{A}-\text { Twist }: & M_{E^{\prime}}=M_{E}+F_{V} \\
\mathrm{~B}-\text { Twist }: & M_{E^{\prime}}=M_{E}+F_{A} .
\end{array}
$$

In the above notation of table 1 the $A$ twist corresponds to a $(-,+)$ twist, i.e. to a combination of the $(-)$ twist on $\psi_{-}, \bar{\psi}_{-}$and the $(+)$-twist on $\psi_{+}, \bar{\psi}_{+}$, while the $B$ twist is $(+,+)$ twist, i.e. a combination of the $(+)$ twist on $\psi_{-}, \bar{\psi}_{-}$and the $(+)$-twist on $\psi_{+}, \bar{\psi}_{+}$. There are the possibilities of an $(+,-)$ twist and an $(-,-)$ twist making $\bar{Q}_{A}$ and $\bar{Q}_{B}$ nilpotent operators. They lead to the definition of conjugated cohomological sectors and correspond to no new theories. However as explained in Sec. 4.5 the combined geometry of the sectors conjugated to each other leads to an interesting geometry, the so called $t t^{*}$ geometry.

The effects on the fields and the supersymmetry transformation can be summarized in the tables 2 and 3 respectively.

As it is clear from the table 3 and $(3.13$ the following combinations

$$
\begin{aligned}
& Q_{A}=Q_{-}+\bar{Q}_{+} \\
& Q_{B}=\bar{Q}_{-}+\bar{Q}_{+}
\end{aligned}
$$

are now scalar, nilpotent operators which can be used to define two different cohomological theories, the topological $A$ - and the topological $B$-model respectively. Mirror symmetry exchanges the 


\begin{tabular}{|c|c|c|c|c|c|c|c|c|c|c|}
\hline \multirow[b]{3}{*}{$x$} & & \multicolumn{3}{|c|}{ Before Twisting } & \multirow{2}{*}{\multicolumn{3}{|c|}{$\begin{array}{r}\text { A twist }(-,+) \\
U(1)_{E}^{\prime} \text { spin }\end{array}$}} & \multirow{2}{*}{\multicolumn{3}{|c|}{$\begin{array}{r}\text { B twist }(+,+) \\
U(1)_{E}^{\prime} \text { spin }\end{array}$}} \\
\hline & \multicolumn{3}{|c|}{$U(1)_{V} U(1)_{A} U(1)_{E}$} & \multirow{2}{*}{$\begin{array}{r}\text { spin } \\
\mathbf{1}_{C}\end{array}$} & & & & & & \\
\hline & 0 & 0 & 0 & & $x$ & 0 & $\mathbf{1}_{C}$ & $x$ & 0 & $\mathbf{1}_{C}$ \\
\hline$\psi_{-}^{i}$ & -1 & 1 & 1 & $K^{\frac{1}{2}}$ & $\chi^{i}$ & 0 & $\mathbf{1}_{C}$ & $\rho_{z}^{i}$ & 2 & $K$ \\
\hline $\bar{\psi}_{+}^{\bar{\imath}}$ & 1 & 1 & -1 & $\bar{K}^{\frac{1}{2}}$ & $\chi^{\bar{l}}$ & 0 & $\mathbf{1}_{C}$ & $-\frac{1}{2}\left(\theta^{\bar{\imath}}+\eta^{\bar{l}}\right)$ & 0 & $\mathbf{1}_{C}$ \\
\hline $\bar{\psi}_{-}^{\bar{\imath}}$ & 1 & -1 & 1 & $K^{\frac{1}{2}}$ & $\rho_{z}^{\bar{l}}$ & 2 & $K$ & $\frac{1}{2}\left(\theta^{\bar{\imath}}-\eta^{\bar{\imath}}\right)$ & 0 & $\mathbf{1}_{C}$ \\
\hline$\psi_{+}^{i}$ & -1 & -1 & -1 & $\bar{K}^{\frac{1}{2}}$ & $\rho_{\bar{z}}^{i}$ & -2 & $\bar{K}$ & $\rho_{\bar{z}}^{i}$ & -2 & $\bar{K}$ \\
\hline
\end{tabular}

Table 2: Space time transformation of the non linear $\sigma$-model fields and charges after $A$ and $B$ twist. We also indicate the names of the fields in the $A$ and $B$ model.

\begin{tabular}{|c|c|c|c|c|c|c|}
\hline \multirow[b]{3}{*}{$Q_{-}$} & \multicolumn{4}{|c|}{ Before Twisting } & \multirow{2}{*}{$\begin{array}{c}\mathrm{A}-\mathrm{twist} \\
U(1)_{E}^{\prime} \text { spin }\end{array}$} & \multirow{2}{*}{$\begin{array}{c}\mathrm{B}-\mathrm{twist} \\
U(1)_{E}^{\prime} \text { spin }\end{array}$} \\
\hline & $U(1)_{V}$ & $(1)_{A}$ & $(1)_{E}$ & spin & & \\
\hline & -1 & 1 & 1 & $K^{\frac{1}{2}}$ & $\begin{array}{ll}0 & \mathbf{1}_{C}\end{array}$ & $2 K$ \\
\hline $\bar{Q}_{+}$ & 1 & 1 & -1 & $\bar{K}^{\frac{1}{2}}$ & $\mathbf{1}_{C}$ & $\mathbf{1}_{C}$ \\
\hline $\bar{Q}_{-}$ & 1 & -1 & 1 & $K^{\frac{1}{2}}$ & $K$ & $\mathbf{1}_{C}$ \\
\hline$Q_{+}$ & -1 & -1 & -1 & $\bar{K}^{\frac{1}{2}}$ & -2 & -2 \\
\hline
\end{tabular}

Table 3: Space time transformation of the supersummetry generators after the $A$ and $B$ twist

- twist with the + twist on the $\psi_{-}, \bar{\psi}_{-}$side. Even before twisting $Q_{A}$ and $Q_{B}$ define cohomological theories on the plane the torus, where covariantly constant spinors exist. One can also choose to twist only the say $\psi_{-}, \bar{\psi}_{-}$side. The indices of so called half-twisted models are the closest analogs of the elliptic genus (3.5) at higher genus [156][161]. This indices are shared between the $A$ and the $B$ model and contain information about the couplings of $1,27,27$ in the heterotic string with standard embedding.

\subsection{Generalities on physical observables}

One calls an operator a chiral operator or $(c, c)$ operator $\phi$ if

$$
\left[Q_{B}, \phi\right]=0 \text {. }
$$

Chiral and twisted chiral superfields play an important rôle in formulating the general $(2,2)$ worldsheet theory, see [162]. The lowest component $\phi$ of chiral superfield $\Phi$ obeys $\left[\bar{Q}_{ \pm}, \phi\right]=0$ and is hence a chiral operator. An operator $\phi$ is called twisted chiral or $(a, c)$ if

$$
\left[Q_{A}, \phi\right]=0 \text {. }
$$

The lowest component $v$ of a twisted chiral superfield $\Sigma$ obeys $\left[\bar{Q}_{+}, v\right]=\left[Q_{-}, v\right]=0$ and is hence a twisted chiral operator. $\left[\bar{Q}_{-}, \phi_{-}\right]=0$ and $\left[Q_{-}, \phi_{-}\right]=0$ define left chiral- and antichiral operators while $\left[\bar{Q}_{+}, \phi_{+}\right]=0$ and $\left[Q_{+}, \phi_{+}\right]=0$ define right chiral- and antichiral operators.

The key concept is now to define a cohomological theory whose observables are the equivalence classes $[\phi]$ of $Q$ closed operators. To be closed the operators have to fulfill $[Q, \phi]=0$ and the 
equivalence relation is as usual up to exact operators $\mathscr{E}=[Q, \Lambda]_{ \pm}$, i.e.

$$
\phi \sim \phi+[Q, \Lambda]_{ \pm}
$$

If the vacuum is annihilated by $Q$, which is the case if $Q$ comes from a unbroken symmetry as above, then the correlation function of the $Q$ closed operators does not depend on the representative of the class

$$
\begin{aligned}
\left\langle\phi_{1} \ldots\left(\phi_{k}+\{Q, \Lambda\}\right) \ldots \phi_{n}\right\rangle= & \left\langle\phi_{1} \ldots \phi_{n}\right\rangle \pm\left\langle 0\left|\phi_{1}, \ldots \phi_{k-1} \Lambda \phi_{k+1} \ldots \phi_{n} Q\right| 0\right\rangle \\
& \mp\left\langle 0\left|Q \phi_{1}, \ldots \phi_{k-1} \Lambda \phi_{k+1} \ldots \phi_{n}\right| 0\right\rangle \\
= & \left\langle\phi_{1} \ldots \phi_{n}\right\rangle
\end{aligned}
$$

The analogy of the definition of topological correlators with cohomological intersections $\int_{M} \omega_{1} \wedge$ $\ldots \wedge\left(\omega_{k}+\mathrm{d} \lambda\right) \wedge \ldots \wedge \omega_{n}=\int_{M} \omega_{1} \wedge \ldots \wedge \omega_{k} \wedge \ldots \wedge \omega_{n}$ is not just formal in the case of the $(2,2)-$ sigma model as we will see.

An important property of these operators is that they form position independent rings. Using the algebra (3.13), the properties of the twisted chiral operators and $[\{A, B\}, C]=\{[A, C], B\}+$ $\{A,[B, C]\}$ it is easy to see that e.g.

$$
\begin{aligned}
& \frac{i}{2}\left(\frac{\partial}{\partial x^{0}}+\frac{\partial}{\partial x^{1}}\right) \phi=[(H+P), \phi]=\left[\left\{Q_{+}, \bar{Q}_{+}\right\}, \phi\right]=\ldots=\left\{Q_{B},\left[Q_{+}, \phi\right]\right\} \\
& \frac{i}{2}\left(\frac{\partial}{\partial x^{0}}-\frac{\partial}{\partial x^{1}}\right) \phi=[(H-P), \phi]=\left[\left\{Q_{-}, \bar{Q}_{-}\right\}, \phi\right]=\ldots=\left\{Q_{B},\left[Q_{-}, \phi\right]\right\}
\end{aligned}
$$

and similar for the $A$ model. Combining (4.8) and (4.9) one sees that the correlation functions of the twisted chiral operators do not depend on the position of the insertions of the operators, which is also true for the chiral operators. The ring structure comes from the operator product expansion. It is obvious that the OPE of two (twisted) chiral fields is (twisted) chiral again and by (4.9) position independent. One defines the structure constants of the ring in a basis of the ring $\phi_{k}$ as

$$
\phi_{i} \phi_{j}=C_{i j}^{k} \phi_{k}+[Q, \Lambda]_{ \pm},
$$

i.e. identifying an element on the right hand side up to exacts term. The ring satisfies the usual associativity $C_{j l}^{m} C_{i k}^{l}=C_{l k}^{m} C_{i j}^{l}$. The unit $\phi_{0}=1$ is always (twisted) chiral, so $C_{0 j}^{k}=C_{j 0}^{k}=\delta_{j}^{k}$.

The position independence (4.9) and its realization on $p$-form operators can be formulated in a covariant way as the so called descend equations, see [40] for a review. If $\mathscr{O}^{(0)}=\phi$ is a $Q$ closed position independent 0 -form operator, one can define the following non-local $n$-form operators

$$
\begin{aligned}
0 & =\left[Q, \mathscr{O}^{(0)}\right] \\
\mathrm{d} \mathscr{O}^{(0)} & =\left\{Q, \mathscr{O}^{(1)}\right\} \\
\mathrm{d} \mathscr{O}^{(1)} & =\left[Q, \mathscr{O}^{(2)}\right] \\
\mathrm{d} \mathscr{O}^{(2)} & =0 .
\end{aligned}
$$

Using (4.9) and the corresponding relation for the $A$-model one can find the descend operators explicitly noting that $Q_{-} \mathrm{d} z\left(\bar{Q}_{-} \mathrm{d} z\right)$ and $Q_{+} \mathrm{d} \bar{z}\left(\bar{Q}_{+} \mathrm{d} \bar{z}\right)$ are covariant combinations

$$
\begin{aligned}
& \text { A-mod. } \mathscr{O}_{A}^{(1)}=i \mathrm{~d} z\left[\bar{Q}_{-}, \mathscr{O}_{A}^{(0)}\right]-i \mathrm{~d} \bar{z}\left[Q_{+}, \mathscr{O}_{A}^{(0)}\right], \mathscr{O}_{A}^{(2)}=\mathrm{d} z \mathrm{~d} \bar{z}\left\{Q_{+},\left[\bar{Q}_{-}, \mathscr{O}_{A}^{(0)}\right]\right\}, \\
& \text { B - mod. } \mathscr{O}_{B}^{(1)}=i \mathrm{~d} z\left[Q_{-}, \mathscr{O}^{(0)}\right]-i \mathrm{~d} \bar{z}\left[Q_{+}, \mathscr{O}_{B}^{(0)}\right], \mathscr{O}_{B}^{(2)}=\mathrm{d} z \mathrm{~d} \bar{z}\left\{Q_{+},\left[Q_{-}, \mathscr{O}_{B}^{(0)}\right]\right\}
\end{aligned}
$$


The descent equations truncate, because of the anti symmetrization in the world-sheet indices. The $\bar{Q}_{B}$ and $\bar{Q}_{A}$ operators define the $(a, a)$ and $(c, a)$ ring states which we call $\overline{\mathscr{O}}_{B}^{(0)}$ and $\overline{\mathscr{O}}_{A}^{(0)}$ respectively. Their descendants $\overline{\mathscr{O}}_{B}^{(1,2)}$ and $\overline{\mathscr{O}}_{A}^{(1,2)}$ are defined as in (4.12) with the barred and unbarred $Q$ operators exchanged. As an easy exercise one checks that $\mathscr{O}_{B}^{(2)}\left(\overline{\mathscr{O}}_{B}^{(2)}\right)$ and $\mathscr{O}_{A}^{(2)} \overline{\mathscr{O}}_{A}^{(2)}$ are $\bar{Q}_{B}\left(Q_{B}\right)$ and $\bar{Q}_{A}\left(Q_{A}\right)$ exact.

The significance of the descendant $p$-form operators is that one can integrate them over closed $p$-cycles $C_{p}$ of the WS (or more general the topological field theory space-time) to obtain nonlocal operators $\mathscr{O}\left(C_{p}\right)=\int_{C_{p}} \mathscr{O}^{(p)}$, which are automatically $Q$ closed, because of Stokes theorem $\left[Q, \mathscr{O}\left(C_{p}\right)\right]_{ \pm}=\int_{C_{p}}\left[Q, \mathscr{O}^{(p)}\right]_{ \pm}=\int_{C_{p}} \mathrm{~d} \mathscr{O}^{(p-1)}=\int_{\partial C_{p}} \mathscr{O}^{(p-1)}=0$. Reversed use of Stokes theorem shows that the topological equivalence class of $\mathscr{O}\left(C_{p}\right)$ depends only the homology class of $C_{p}$. For a $p-1$ chain $S$ with $C_{p}-C_{p}^{\prime}=\partial S$ the difference $\mathscr{O}\left(C_{p}\right)-\mathscr{O}\left(C_{p}^{\prime}\right)=\int_{\partial S} \mathscr{O}^{(p)}=\int_{S} \mathrm{~d} \mathscr{O}^{(p)}=$ $\left[Q, \int_{S} \mathscr{O}^{(p+1)}\right]_{ \pm}$is $Q$ exact. As we shall see we have $\mathscr{O}_{W_{(1,1)}}^{(0)}=w_{i j} \chi^{i} \chi^{\bar{j}}$ operators in the $A$ model associated to elements in $H^{1,1}(M) 5.3,5.4$ ), which have according to table $2\left(U(1)_{V}, U(1)_{A}\right)$ charges $(0,2)$. These charges are offset by $Q_{+}, \bar{Q}_{-}$, as seen from table (3) so that $\mathscr{O}_{W_{(1,1)}^{(2)}}$ is neutral. Similarly the operators associated to elements in $A \in H^{1}(M, T M)(6.9)$ in the B-model $\mathscr{O}_{A}^{(0)}=w_{j}^{i} \eta^{\bar{j}} \theta_{i}$ have $\left(U(1)_{V}, U(1)_{A}\right)$ charges $(2,0)$ which is offset by $Q_{+}, Q_{-}$so that $\mathscr{O}_{A}^{(2)}$ is neutral. Neutrality of these operators means that we can add them in arbitrary numbers to correlations functions without affecting the selections rules.

\subsection{A first look at the metric (in)dependence and topological string theory}

In a topological theory the correlation functions are not only formally position independent, but decouple formally from variations of the worldsheet metric $h^{\mu v}$. Classically the energy momentum tensor $T_{\mu \nu}=\frac{1}{\sqrt{h}} \frac{\delta S}{\delta h^{\mu \nu}}$ is the generator of those variations. From the first order variation of the weight factor $e^{S}$ one gets a dependence of a correlation function on metric variations $\delta h^{\mu v}$

$$
\delta_{h}\langle\mathscr{O}\rangle_{g}=\left\langle\mathscr{O} \int_{\Sigma_{g}} \sqrt{h} \mathrm{~d}^{2} \sigma \delta h^{\mu v} T_{\mu v}\right\rangle_{g} .
$$

In a topological theory $\delta_{h}\langle\mathscr{O}\rangle_{g}=0$ does not require that $T_{\mu v}=0$ but in virtue of (4.8) that it is exact

$$
T_{\mu v}=\left\{Q, G_{\mu v}\right\}
$$

This structure ensures general covariance or topological invariance. It plays a key role in covariant quantization of string theory, where $Q^{2}=0$ is the BRST operator and the part of $G_{\mu \nu}$ is played by the antighost field $b_{\mu \nu}$. It is also the starting point of closed string field theory formulations [148]. One can have topological invariance independently of conformal invariance and also independently of the decoupling between ghost and matter sector [148]. For instance the $A$ model relies on this structure and can be defined on Kähler manifolds on which the $\sigma$ model is not conformally invariant.

In string theory we integrate the world-sheet metric $h$ of $\Sigma_{g}$ over all possible choices $\mathscr{H}_{g}$. [126] is the standard extended reference for the following short review of the metric dependence. Classically the integral over $h$ is invariant under diffeomorphism and Weyl- and conformal transformations of the metric $\tilde{h}_{a b}(\tilde{\sigma})=\exp [2 \omega(\sigma)] \frac{\partial \sigma^{c}}{\partial \tilde{\sigma}_{a}} \frac{\partial \sigma^{d}}{\partial \tilde{\sigma}_{b}} h_{c d}$. These "gauge" invariances are present 
at quantum level in critical string theory, which does not require an anomaly cancellation for the latter. The integral over the metric hence contains a gauge orbit over the diffeomorphism- and the Weyl group, which requires a gauge fixing. After this gauge fixing the reduced moduli space is ${ }^{4}$

$$
\mathscr{M}_{g}=\text { large gauge transf. } \backslash \mathscr{H}_{g} /(\text { diff } \times \text { Weyl })_{g} .
$$

Large gauge transformations refer to diffeomorphism of $\Sigma_{g}$ not connected to the identity, i.e. the mapping class group, which does not affect the dimension or other local properties of $\mathscr{M}_{\mathrm{g}}$. Let us focus on the latter, which are described by those infinitesimal transformations $\tilde{\delta} h^{a b}$ which are orthogonal to the infinitesimal Weyl and diffeomorphism transformations

$$
\delta h_{a b}=2 \delta \omega h_{a b}-\nabla_{a} \delta \sigma_{b}-\nabla_{b} \delta \sigma_{a},
$$

in the sense that $\int_{\Sigma_{g}} \mathrm{~d} \sigma^{2} \sqrt{h} \tilde{\delta} h^{a b} \delta h_{a b}=0$. It is not hard to see [55], [126] (Vol. I) that these deformations of the metric correspond to elements $\mu_{\bar{z}}^{z} \mathrm{~d} z \frac{\partial}{\partial z} \in H^{1}(T \Sigma)$. As explained in Sec. 6.2 this cohomology group describes the independent first order complex structure deformations of $\Sigma$. We have to take the cohomology group to exclude changes of the metric by reparametrizations. On the other hand there are certain reparametrizations, which do not change the metric. Reparametrizations are locally described by vector fields and currently we are looking for those that do not change the conformal class of the metric. These are the conformal Killing fields, elements in $H^{0}(T \Sigma)$. They are canceled from the denominator of (4.15). Hence the expected dimension of $\mathscr{M}_{g}$ is $h^{1}(T \Sigma)-h^{0}(T \Sigma)$, which we calculated in Sec. (7.3) to be $3 g-3$. To avoid the peculiarities of $h^{0}(T \Sigma) \neq 0$ ( 3 and 1 for $g=0$ and $g=1$ ) consider $g>1$ and let $z^{a}=: m^{a}, a=1, \ldots, 3 g-3$ the complex structure variables of $\Sigma$. We can describe then a first oder deformation of the metric modulo Weyl and diffeomorphisms [55] as $\int_{\Sigma} \mathrm{d}^{2} \sigma \sqrt{h} \tilde{\delta} h^{a b} T_{a b}=\int_{\Sigma} \mathrm{d}^{2} z \mu_{\bar{z}}^{(a) z} \delta m^{a} T_{z z}+\bar{\mu}_{z}^{a \bar{z}} \delta \bar{m}^{a} \bar{T}_{\bar{z} z}$ and if we insert that in (4.13) we conclude that

$$
\frac{\partial}{\partial m^{a}}\langle\mathscr{O}\rangle_{g}=\left\langle\mathscr{O} \int_{\Sigma} \mathrm{d}^{2} z \mu_{\bar{z}}^{a z} T_{z z}\right\rangle_{g}=:\left\langle\mathscr{O} T^{a}\right\rangle_{g}
$$

and similarly $\frac{\partial}{\partial \bar{m}^{a}}=\left\langle\mathscr{O} \bar{T}^{a}\right\rangle_{g}$. Eq. 4.14 is strictly true, so the argument that cohomological states and the vacuum are $Q$ closed would make topological string theory completely metric independent and therefore trivial! However the argument involving the invariance of the vacuum fails, because the measure on the moduli space of higher genus Riemann surfaces, which is part of the vacuum definition is not $Q$ closed. It is a real $6 g-6$ form $\mu_{g}$ for surfaces of $g>1$ and the argument fails in a very specific way. If we act with $Q$ on it, it gives an exact form, as we will see in detail in Sec. 6.11. This is like a descent equation, but with exterior derivative in the moduli space direction. By Stokes or rather Dolbeaults theorem the contribution to the integral can then only come from the boundary of $\mathscr{M}_{g}$, which represents degenerate Riemann surfaces. If the vacuum is not $Q$ closed we cannot trust the argument about position independence either. In the moduli space $\mathscr{M}_{g, n}$ with insertion of $n$ operators the codimension one locus, where two operators coincide, is part of the boundary components. Its contributions has to be taken into account by so called contact terms. Most of what topological string theory is about is organizing the contributions of these boundaries.

\footnotetext{
${ }^{4}$ In case of $n$ operator insertions the moduli space is extended through the positions of the points by $n$ complex dimensions to $\mathscr{M}_{g, n}$.
} 
The question which boundaries do give contributions leads to the stable compactifications on $\overline{\mathscr{M}}_{g, n}$ in which only the boundary components are included, which are in complex codimension one. These facts will govern the coupling of the $A$ and the $B$-model to WS gravity as discussed in Sec. 5.2 and 6.11 .

\subsection{A first look at the deformation space}

What is of importance is that integrals of the two form operators $\int_{\Sigma} \mathscr{O}_{i}^{(2)}$ defined in the Sec. 4.1 can be added to the topological action as deformations

$$
S=\int_{\Sigma} \mathrm{d} z^{2} \mathscr{L}_{0}+\sum_{i=1}^{r} t^{i} \int_{\Sigma} \mathscr{O}_{i}^{(2)}
$$

This might extend the theory to a family of theories and we expect that the neutral operators we discussed at the end of the last section lead to non-trivial deformation families. The reason is that arbitrary derivatives of a correlation function w.r.t. $t_{i}$ might be non-trivial. Such derivatives bring down $U(1)_{A / V}$ neutral operators in the path integral which do not affect the $U(1)_{A / V}$ selection rules and may all not vanish. An early world-sheet argument for the existence of such deformation families was given in [45].

In conformal field theory operators $\mathscr{O}^{(1,1)}$ having conformal dimension $(h, \bar{h})=(1,1)$ are called marginal operators. $(h, \bar{h})=(1,1)$ is a first order condition in $t$ for 4.18 to define a conformal theory. It is far from trivial that this is the case for finite deformations in $t$. Operators for which this is the case are called exactly marginal. It should be stressed that the topological models allow in our context for more general perturbations then the CFT. The reason is that by (4.28) all operators that obey the relation $h=\frac{|Q|}{2}$ between its $U(1)$ charge $Q$ and its conformal dimension $h$, can become a scalar operator $\mathscr{O}^{(0)}$ w.r.t. $\hat{T}$ after a suitable twist. Then an $\mathscr{O}^{(2)}$ can be associated to it by the descend relations. However not all such deformation operators started out as marginal operators $\mathscr{O}^{(1,1)}$ in the CFT. In this lecture we will focus on the deformations, which preserve the conformal symmetry on the WS. As we will see in Sec.s 5.1 and 6.1 these are only a subset of the $\mathscr{O}^{(2)}$ operators in the cohomological field theory. Perturbation w.r.t. the full set of perturbations has been considered in [9].

It is interesting to recover this first order condition of the CFT from the spacetime point of view, see [23, 22]. We know that the geometrical background has to be Calabi-Yau manifold to allow for a conformal field theory ${ }^{5}$. The exactly marginal deformations $\mathscr{O}^{(1,1)}$ must correspond hence to deformations of the geometry, which preserve the Calabi-Yau condition. I.e. to deformations of the background metric $g_{\mu \nu}+\delta g_{\mu, v}$ (and B-field $b_{\mu \nu}+\delta b_{\mu \nu}$ ), which do not change the Calabi-Yau condition ${ }^{6} R_{\mu \nu}(g)=0$, i.e.

$$
R_{\mu v}(g+\delta g)=0 .
$$

In analyzing this equation we have to eliminate the $\delta g$, which come from coordinate transformations. Coordinate transformations or equivalently diffeomorphism of $M$ are generated by vectors

\footnotetext{
${ }^{5}$ There is an interesting extension of these considerations for non-conformal $N=(2,2) \sigma$-models involving massive (non-marginal) deformations.

${ }^{6}$ Strictly speaking one should ask for perturbations, which leave the Ricci-form $\mathscr{R}$ in the $c_{1}(M)=0$ cohomology class. Though the representatives of the deformations in the cohomology classes would be different, the counting would be the same, see Sec. 7.5 .
} 
fields $V^{\mu}$, compare Sec. 6.2. An actual change of the metric $\delta g_{\mu \nu}$ is orthogonal to diffeomorphism generated by the vector field in the following sense $\int \sqrt{g} \delta g^{\mu \nu}\left(\nabla_{\mu} V_{v}+\nabla_{v} V_{\mu}\right) \mathrm{d}^{m} x=0$, which is equivalent to the gauge condition $\nabla^{\mu} \delta g_{\mu \nu}=0$. Expanding with this constraint (4.19) to linear order around $R(g)=0$ one gets

$$
\nabla^{\rho} \nabla_{\rho} \delta g_{\mu v}-2 R_{\mu}{ }^{\kappa}{ }_{v}{ }^{\sigma} \delta g_{\kappa \sigma}=0
$$

Using the splitting of a Kähler metric in holomorphic and holomorphic indices one can analyze $\delta g_{i \bar{j}}$, and $\delta g_{i j}$ separately. Note that $\delta g_{i \bar{j}}$ is real, while $\delta g_{i j}$ with $\overline{\delta g_{i j}}=\delta g_{\bar{\imath} \bar{j}}$ is complex. From (7.27) it follows that $\delta g_{i \bar{j}}$ is $\Delta_{d}$ harmonic and $\delta g^{i}=\delta g_{\bar{j}}^{i} \mathrm{~d} z^{\bar{j}}=g^{i \bar{k}} \delta g_{\bar{k} \bar{j}} \mathrm{~d} z^{\bar{j}}$ is $\Delta_{\bar{\jmath}}$ harmonic. In other words the first order deformations factorize and correspond to elements in $H^{1,1}(M)$ and $H^{1}(M, T M)$ respectively. These are also among the deformations of the $A$ - and $B$-model as mentioned above and further discussed in the following Sec. 5.1 and 6.1.

Let us first discuss the two moduli space associated to $H^{1,1}(M)$. In a basis of $(1,1)$-forms $\omega_{(1,1)}^{(k)}$, we expand a Kähler form

$$
\omega=\sum_{k=1}^{h^{11}} t_{k} \omega_{(1,1)}^{(k)}
$$

in terms of the real Kähler parameters $t_{k}>0$. The range of $t_{k}$ is bounded by the inequalities, which ensure positivity of the volumes of curves $C$, divisors $D$ and $M$, i.e.

$$
\int_{C} \omega>0, \quad \int_{D} \omega \wedge \omega>0, \quad \int_{M} \omega \wedge \omega \wedge \omega>0
$$

These conditions describe a real cone in $\mathbf{R}_{+}^{h^{1,1}}$, which is called the Kähler cone. The parameters $t_{k}$ are identified with the areas of dual curves $C_{k}$ to $\omega_{(1,1)}^{(k)}$, which shrink to zero area at the boundaries of the Kähler cones ${ }^{7}$. In the $\sigma$-model (5.1) it is natural to complexify the parameter $t_{k}$ to $t_{k}^{\sigma}=$ $\int_{C_{k}}(\omega-i B)$ by adding the integral of the antisymmetric tensor field $B \in H^{1,1}(M)$ to $t_{k}$. Moreover due to mirror symmetry one has a natural choice of the complex parametrization of the complexified Kähler moduli space $\mathscr{M}_{K}$, simply the complex structure parameters of the mirror $t_{k}^{m 8}$

As it is clear from the fact that the deformations $\delta g_{i j}, \delta g_{\bar{l} \bar{j}}$ change the $(i, \bar{l})$ type of the metric, the moduli space $H^{1}(M, T M)$ is associated to complex structure deformations. It is fair to say that most of what we know about the moduli space of $(2,2)$ theories comes from the theory of complex structure deformations. In particular it can be shown that the first order deformations of the complex structures elevate to finite deformations. This is more thoroughly discussed in the Sec. 6.2 and 6.3 .

Let us conclude the description of emerging picture of the deformation spaces. We have found that the $U(1)_{A / V}$ neutral world sheet two form operators $\mathscr{O}_{\omega_{(1,1)}}^{(2)}$ with $\omega_{1,1} \in H^{1,1}(M, Z)$ and $\mathscr{O}_{A}^{(2)}$ with $A \in H^{1}(M, T M)$ correspond geometrically to complexified Kähler and complex structure deformations of the Calabi-Yau metric and are expected to be exactly marginal from the CFT point of view. In the low energy effective action of type II A/B string theory these marginal deformations

\footnotetext{
${ }^{7}$ At the boundary of the Kähler also a divisor may collapse. In this case $t_{k}$ is still the area of a curve $C_{k}$ in $D$.

${ }^{8}$ As a corollary all singularities of $\mathscr{M}_{K}$ occur at complex codimension one and the cone structure disappears completely.
} 
arise as vacuum expectation of complex scalar fields labeling the vacuum manifold of the $\mathrm{N}=2$ supergravity in $4 \mathrm{~d}$. The general structure of this vacuum manifold for abelian gauge groups $U(1)^{\# V}$ and $U(1)^{\# H}$ is that it is locally of the form $\mathscr{M}_{2 \# V} \times \mathscr{Q}_{4 \# H}$, where $\mathscr{M}$ is a complex special Kähler manifold for the scalar fields in the vector multiplets[37][38][35] and $\mathscr{Q}$ is a quaternionic manifold [28] for the scalar fields in the hypermultiplets. The subscripts indicate the real dimension of the moduli space. Its relation to the perturbative sector of the II A/B string compactifications on a Calabi-Yau 3 fold $M$ is as follows

$$
\mathscr{M}_{t o t}^{I I A}(M)=\mathscr{M}_{2 h^{1,1}(M)}^{I I A} \times \mathscr{Q}_{4\left(h^{2,1}(M)+1\right)}^{I I A} \quad \mathscr{M}_{t o t}^{I I B}(W)=\mathscr{M}_{2 h^{2,1}(W)}^{I I B} \times \mathscr{Q}_{4\left(h^{1,1}(W)+1\right)}^{I I B} .
$$

One very far reaching definition of the mirror conjecture is that type IIA and type IIB compatifications are completely identically if $M$ and $W$ are mirror pairs. This in particular implies $\mathscr{M}_{\text {tot }}^{I I A}(M)=\mathscr{M}_{\text {tot }}^{I I A}(W)$. The best studied object is $\mathscr{M}_{2 h^{2,1}(W)}^{I I B}$ since it is literally the complex moduli space of $W$. The enhancement of the Calabi-Yau metric moduli space from the complex to the quaternionic space $\mathscr{Q}$ of Kähler multiplets is due to the moduli of Ramond forms. The additional quaternionic dimension in $\mathscr{Q}$ comes from the universal dilation, whose scalar components $(S, C)$ contain in particular the type II dilation $S$.

\subsection{Conformal Field Theory point of view}

A most remarkable fact is that for all 145 Calabi-Yau threefolds defined in weighted projective space subject to the constraint (7.61) and for which the defining polynomial is of Fermat type

$$
P=\sum_{i=1}^{5} a_{i} x^{m_{i}}
$$

with $m_{i} w_{i}=d, \forall i$ and $\sum_{i=1}^{5} w_{i}=d$ there is a well founded conjecture for an exact conformal field theory description, which captures the full perturbative sector and not just the topological part of it. The CFT description is based on an orbifold of tensor products of minimal $N=2$ super conformal field theories found by Gepner [61]. The description is valid only at one point in complex structure and complexified Kähler structure moduli space the so called Gepner point. In the complex moduli space the constraint (4.24) literally describes this special point. In the complexified Kähler moduli the point can also be described by (4.24) after dividing by phase symmetry groups such as 6.56,6.65), which identifies (4.24) with the mirror manifold. It is far away from the large volume limit.

The purpose of the present section is to describe the topological sub sectors in CFT language and to link them to the full perturbative spectrum of the string.

As it is well known [126] Vol. II $N=2$ supergravity and $N=1$ heterotic string $E_{8} \times E_{8}$ string compactifications with standard embedding require an $N=(2,2)$ supersymmetry. Only a $N=$ $(1,1)$ symmetry is gauged. The $N=2$ chiral part of a superconformal algebra on the worldsheet has beside the chiral component of energy momentum tensor ${ }^{9} T(z)=\sum_{n \in Z} \frac{L_{n}}{z^{n+2}}$ with conformal

\footnotetext{
${ }^{9}$ The standard notation in CFT is quite different than the one common in the discussion of $\sigma$ models that we used in Sec. 3. One uses in CFT $z=\sigma^{1}+i \sigma^{2}$ and $\bar{z}=\sigma^{1}+i \sigma^{2}$ where $\sigma^{2}=i \sigma^{0}$ is the euclidean time. Correspondingly one indicates the left moving sector which carried a + index in Sec. 3 by quantities without bar and the right moving carrying before - with quantities with bar. Moreover the unbarred or barred super charges are now distinguished by and + respectively, e.g. $Q_{+} \leftrightarrow G_{0}^{-}, \bar{Q}_{+} \leftrightarrow G_{0}^{+}, Q_{-} \leftrightarrow \bar{G}_{0}^{-}$and $\bar{Q}_{-} \leftrightarrow \bar{G}_{0}^{+}$.
} 


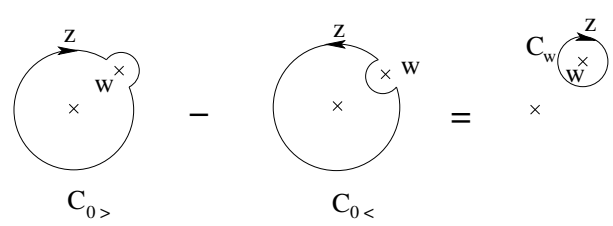

Figure 2: ???

dimension and $U(1)$ charge $(h, Q)=(2,0)$ an $U(1)$ current $J(z)=\sum_{n \in Z} \frac{J_{n}}{z^{n+1}}$ with $(h, Q)=(1,0)$ and two super currents $G^{ \pm}=\sum_{r \in Z \pm v} \frac{G_{r}^{ \pm}}{z^{r+\frac{3}{2}}}$ with $(h, Q)=\left(\frac{3}{2}, \pm 1\right)$. The shift $v$ can take arbitrary real values. The short distance operator expansion is

$$
\begin{aligned}
T(z) T(0) & =\frac{c}{2 z^{4}}+\frac{2}{z^{2}} T(0)+\frac{1}{z} \partial T(0), \\
T(z) G^{ \pm}(0) & \sim \frac{3}{2 z^{2}} G^{ \pm}(0)+\frac{1}{z} \partial G^{ \pm}(0), \\
T(z) J(0) & \sim \frac{1}{z^{2}} J(0)+\frac{1}{z} \partial J(0), \\
G^{+}(z) G^{-}(0) & \sim \frac{2 c}{3 z^{3}}+\frac{2}{z^{2}} J(0)+\frac{2}{z} T(0)+\frac{1}{z} \partial J(0), \\
G^{+}(z) G^{+}(0) & \sim G^{-}(z) G^{-}(0) \sim 0, \\
J(z) G^{ \pm}(0) & \sim \pm \frac{1}{z} G^{ \pm}(0), \\
J(z) J(0) & \sim \frac{c}{3 z^{2}},
\end{aligned}
$$

Let us recapitulate the standard procedure in 2d QFT which recovers the algebra of charge operators from an operator algebra such as (4.25). To the operator $A(z)$ we assign charge operators $A_{\xi}=$ $\oint_{C_{0}} \mathrm{~d} z \xi(z) A(z)$, where $C_{0}$ is a contour around the origin 0 and $\oint_{C_{0}} \mathrm{~d} z:=\int_{C_{0}} \frac{\mathrm{d} z}{2 \pi i}$. In particular for $\xi(z)=z^{n+h(A)-1}$ the charges are the modes $A_{n}$ of $A(z)$. The transformation of the operator $B(w)$ under $\left(\delta_{A_{\xi}}\right)$ is generated by the commutator with $A_{\xi}$. In radial time ordering the commutator is given by the following contour integrals

$$
\begin{aligned}
\left(\delta_{A_{\xi}}\right) B(w) & =\left[A_{\xi}, B(w)\right]=\oint_{\substack{C_{0} \\
|z|>|w|}} \mathrm{d} z \xi(z) A(z) B(w)-\oint_{\substack{C_{0} \\
|z|<|w|}} \mathrm{d} z \xi(z) A(z) B(w) \\
& =\oint_{C_{w}} \mathrm{~d} z \xi(z) A(z) B(w)
\end{aligned}
$$

see Fig. 2. The spatial transformations $\delta_{\xi}$ corresponding to conformal transformations ${ }^{10} z \rightarrow$ $z+\xi(z)$ are generated by $T(z)$, i.e. $\delta_{\xi}=\delta_{T_{\xi}}$. One can integrate 4.26$)$ with $\oint_{C_{w=0}^{\prime}} \mathrm{d} w z^{m+h(B)-1}$ to

\footnotetext{
${ }^{10}$ These are holomorphic in $2 \mathrm{~d}$.
} 
recover as residue the mode algebra from

$$
\begin{aligned}
{\left[L_{m}, L_{n}\right] } & =(m-n) L_{m+n}+\frac{c}{12} m\left(m^{2}-1\right) \delta_{m,-n}, \\
{\left[L_{m}, G_{r}^{ \pm}\right] } & =\left(\frac{m}{2}-r\right) G_{m+r}^{ \pm}, \\
{\left[L_{m}, J_{n}\right] } & =-n J_{m+n}, \\
\left\{G_{r}^{+} G_{s}^{-}\right\} & =2 L_{r+s}+(r-s) J_{r+s}+\frac{c}{3}\left(r^{2}-\frac{1}{4}\right) \delta_{r,-s}, \\
\left\{G_{r}^{+}, G_{s}^{+}\right\} & =\left\{G_{r}^{-}, G_{s}^{-}\right\}=0, \\
{\left[J_{n}, G_{r}^{ \pm}\right] } & = \pm G_{r+n}^{ \pm}, \\
{\left[J_{m}, J_{n}\right] } & =\frac{c}{3} \delta_{m,-n},
\end{aligned}
$$

with $L_{n}^{\dagger}=L_{-n}, J_{n}^{\dagger}=J_{n}$ and $\left(G_{r}^{ \pm}\right)^{\dagger}=G_{-r}^{\mp}$. In case that the $N=(2,2)$ CFT theory is the internal part of a string compactification it must have $c=\bar{c}=9$ to cancel the Weyl anomaly. It represents the internal manifold $M$. In fact $d:=\operatorname{dim} C(M)=\frac{c}{3}$. The generalized GSO projection restricts the internal $U(1)$ charges to odd integer values for space time bosons and half integer values for space time fermions, see [61, 126] for more details.

If we consider now the $(+,-)$ twisting ${ }^{11}$ [49] 40 ]

$$
\hat{T}(z)=T(z) \pm^{\prime} \frac{1}{2} \partial J(z) \quad \rightarrow \quad \hat{L}_{0}=L_{0} \pm^{\prime} \frac{1}{2} J_{0}
$$

then the modifications of 4.25 occur in the following short distance expansions

$$
\begin{aligned}
\hat{T}(z) \hat{T}(0) & \sim \frac{2}{z^{2}} \hat{T}(0)+\frac{1}{z} \partial \hat{T}(0) \\
\hat{T}(z) G^{ \pm}(0) & \sim \frac{3 \pm^{\prime} \mp 1}{2 z^{2}} G^{ \pm}(0)+\frac{1}{z} \partial G^{ \pm}(0) \\
\hat{T}(z) J(0) & \sim \frac{1}{z^{2}} J(0)+\frac{1}{z^{2}} \partial J(0) \mp^{\prime} \frac{c}{3 z^{3}}, \\
G^{+}(z) G^{-}(0) & \sim \frac{2 c}{3 z^{3}}+\frac{2}{z} J(0)+\frac{2}{z} \hat{T}(0)+\frac{1 \mp^{\prime} 1}{z} \partial J(0) .
\end{aligned}
$$

Let us point out the salient features of the operator product expansions in (4.29)

- Since the central term in the first OPE vanishes no ghost system is required to quantize the world sheet theory.

- By the second OPE either $G^{+}$(+-twist) or $G^{-}$(--twist) become a spin one currents, so either $Q=G_{0}^{+}=\oint G^{+}$or $G_{0}^{-}=\oint G^{-}$becomes conformal, i.e. scalars that are defined on every genus world sheet. The opposite super currents $G^{-}$(+-twist) or $G^{+}$(--twist), become spin 2 fields.

- The above conformal zero modes are recognized as building blocks for nilpotent operators $Q_{A / B} \cdot Q_{A}=G_{0}^{+}+\bar{G}_{0}^{-}$in the case of the $(+,-)$twist defining the $(c, a)$ twisted chiral ring as cohomology. $Q_{B}=G_{0}^{+}+\bar{G}_{0}^{+}$for the $(+,+)$twist defining the $(c, c)$ chiral ring. The relation

\footnotetext{
${ }^{11} \pm^{\prime}$ marked by a prime are correlated in 4.14 .29 .
} 
to geometry of $M$ is ${ }^{12}$ for the $A$-model $Q_{A} \leftrightarrow \mathrm{d}$ and the for the $B$-model $Q_{B} \leftrightarrow \bar{\partial}$ as discussed in more detail in the Sec. 5.1, 6.1.

- The third OPE shows that $J(z)$ has an anomalous transformation. By arguments familiar from the BRST quantization of the bosonic string this gives rise to an anomaly in the divergence of the current, which can be covariantly written as

$$
\nabla^{\mu} J_{\mu}=-\frac{d}{2 \pi} \sqrt{h} R=-d c_{1}\left(\Sigma_{g}\right)=d(2 g-2) .
$$

For $d=\frac{c}{3}=3$ this comes precisely with the same anomalous coefficient -3 as the ghost current in the BRST quantization of the bosonic string $j_{g}=-: b c:$, see [126]. Integrating the anomaly in the divergence of the current leads to a $U(1)$-charge violation of $d(2 g-2)$ on a genus $g$ Riemann surface.

- The last OPE finally is like the one between the BRST current and the $b$ ghost. Integration around a contour to isolate $G_{0}^{+}$, yields for the + twist

$$
\left\{Q, G^{-}(z)\right\}=T(z),
$$

which echos the main equation $\left\{Q_{B R S T}, b(z)\right\}=T^{g+m}(z)$ in the BRST quantization of the bosonic string. We have seen already that $G^{-}$has $(h, Q)=(2,-1)$, which are precisely the conformal dimension and ghost charge of the $b(z)$ ghost.

To summarize we have for the $(+,+)$ twist [11] exactly the same structure as in the bosonic string if we identify

$$
\left(G^{+}(z), J(z), T(z), G^{-}(z)\right) \leftrightarrow\left(J_{B R S T}(z), j_{g}=-: b c:(z), T^{m+g}(z), b(z)\right)
$$

and similar for the anti chiral half. This implies also $Q_{B} \leftrightarrow Q_{B R S T}$ and the ghost number becomes $U(1)_{A}$ charge.

The degenerate ground states in the Ramond-Ramond sector fulfill [108]

$$
G_{0}^{ \pm}|\psi\rangle=0 .
$$

These Ramond-Ramond ground states have by (4.27)

$$
h=\frac{c}{24}=\frac{3}{8} \text {. }
$$

An operator $\mathscr{O}$ with charge $Q$ in the theory can be decomposed into a part $\hat{\mathscr{O}}$ which is neutral under the $U(1)$ current and a charge carrying part, i.e. $\mathscr{O}=\hat{\mathscr{O}} e^{i Q \sqrt{\frac{3}{c}} \phi}$, where we bosonize the current as $J=\sqrt{\frac{c}{3}} \partial \phi[130,108]$. Hence there is a natural operation, which shifts the $U(1)$ charge of every operator $e^{i Q \sqrt{\frac{3}{c}} \phi} \rightarrow e^{i(Q-a) \sqrt{\frac{3}{c}} \phi}$. It is easy to see that this operation induces a family of algebra automorphisms known as spectral flow [130]

$$
\begin{aligned}
L_{n} \rightarrow L_{n}^{\prime} & =L_{n}+a J_{n}+\frac{1}{6} a^{2} c \delta_{n, 0} \\
J_{n} \rightarrow J_{n}^{\prime} & =J_{n}+\frac{1}{3} a c \delta_{n, 0} \\
G_{r}^{ \pm} \rightarrow\left(G_{r}^{ \pm}\right)^{\prime} & =G_{r \mp a}^{ \pm} .
\end{aligned}
$$

\footnotetext{
${ }^{12}$ For Calabi-Yau manifolds this identifications can be viewed as convention and is reversed in [11].
} 
The Ramond ground states are related by (4.35) with $a= \pm^{\prime} \frac{1}{2}$ to states in the NS sector with

$$
G_{r}^{ \pm}|\psi\rangle=L_{n}|\psi\rangle=J_{n}|\psi\rangle=0, \quad r>0, \quad n>0, \quad \text { and } G_{-\frac{1}{2}}^{ \pm^{\prime}}|\psi\rangle=0
$$

Only the $\pm^{\prime}$ in (4.36) correlates with the one in $a= \pm^{\prime} \frac{1}{2}$ and one has $(+)$ for chiral and $(-1)$ for anti chiral states. It is easy to see that $4.36,4.27$ imply

$$
h= \pm \frac{1}{2} Q, \quad|Q| \leq \frac{c}{3}=d .
$$

Massless space-time scalars have $(Q, \bar{Q})=( \pm 1, \pm 1)$. The states in the chiral- and anti chiral rings with this property are related to the cohomology of $M$. The $(c, c)$ ring corresponds to $H^{2,1}(M)$ and the $(c, a)$ ring corresponds ${ }^{13}$ to $H^{1,1}(M)$. The above spectral flow operators with $a= \pm \frac{1}{2}$ relate space time superpartners with each other and are identified with internal part of the spacetime susy operators [61].

The main point in Gepners construction is to identify the internal $c=\bar{c}=9$ theory with an orbifold of a tensor product of minimal $(2,2)$ superconformal field theories. The factor theories are constructed as cosets of supersymmetric, WZW models, see [92] for a general discussion. WZW models and cosets are an important source of rational CFT beyond $c>1$. In the simplest case based on a $(S U(2) \times U(1)) / U(1)$ coset the central charge is

$$
c_{k}=\frac{3 k}{k+3}, \quad k \in N \text {. }
$$

Primary states $|l, q, s\rangle$ of the algebra (4.25) are labeled in the minimal models by integers which have the following standard range ${ }^{14}$

$$
\begin{aligned}
& 0 \leq l \leq k, \\
& 0 \leq|q-s| \leq l \\
& s=\left\{\begin{array}{ll}
0,2 & \text { Neveu }- \text { Schwarz }- \text { sector } \\
\pm 1 & \text { Ramond }- \text { sector }
\end{array}\right\} \\
& l+q+s=0 \bmod 2
\end{aligned}
$$

and have conformal dimension and charge

$$
h=\frac{l(l+2)}{4(k+2)-q^{2}}+\frac{s^{2}}{8}, \quad Q=-\frac{q}{k+2}+\frac{s}{2} .
$$

Above we discussed only the right moving part of the theory. There is a remarkable $A-D-E$ classification, behind the question how to combine the $\chi_{l, q, s}$ and $\chi_{\bar{l}, q, s}$ characters to a modular invariant one loop partition function [20]. Note that above only $l \neq \bar{l}$. That is because all possible shifts of $q, s$ w.r.t. $\bar{q}, \bar{s}$ are obtainable in a separate step by orbifold constructions w.r.t. to simple current symmetries. The simplest way to get a modular invariant theory is to start with a left right symmetric theory with states $|l, q, s ; l, q, s\rangle$, this corresponds to the $A$-series. Considering only this

\footnotetext{
${ }^{13}$ The $(a, a)$ and $(a, c)$ rings correspond to conjugated fields and contain no independent information.

${ }^{14}$ For the orbifold procedure the following equivalences are important $q \sim q \bmod 2(k+2), s=s \bmod 4$ and $|l, q, s ; \bar{l}, \bar{q}, \bar{s}\rangle \sim|k-l, q, s ; k-\bar{l}, \bar{q}+k+2, \bar{s}+2\rangle$.
} 
series there are 145 possibilities to build a tensor product theory with $\bar{c}=c=\sum_{i=1}^{5} c_{k_{i}}=9$. Note that at most one $k_{j}$ is allowed to be zero, because of the $c=9$ condition. This is the same number as $c_{1}\left(T_{M}\right)=0$ Fermat hypersurfaces in $W C P^{4}$, i.e. with $\sum_{i=1} w_{i}=d$, see Sec. 7.7. In fact identifying $m_{i}=d / w_{i}=k_{i}+2$ it is easy to see that both enumerations lead to the same diophantic problem. The simplest possibility is $k_{i}=3$ for $i=1, \ldots, 5$. This leads to $d=5, w_{i}=1, i=1, \ldots 5$, the Quintic in $\mathbf{P}^{4}$. Gepners orbifold construction divides the symmetric tensor product by a symmetry group which is generically the subgroup $G=\mathbf{Z}_{\text {least com. mult. }\left\{k_{i}\right\}} \times\left(\mathbf{Z}_{2}\right)^{r+1}$ among the group generated by the simple currents and constructs a modular invariant orbifold. The effect is that the factor theories and the space-time part are either all in the NS-NS sector or all in the R-R sector and that the charges in the internal NS-NS sector become odd integers [61, 62]. It is then easy to see that states in $(c, c)$ ring from the invariant sector ${ }^{15}$ of the orbifold are of the form $\bigotimes_{i}\left|l_{i}, l_{i}, 0 ; l_{i}, l_{i}, 0\right\rangle$. For the tensor product model that corresponds to the quintic this leads in view of (4.39) to 101 elements. The counting is the same that leads to the 101 independent complex structure deformations under Eq. (6.55), which are identified with elements in $H^{2,1}(M)$. All states in the $(a, c)$ ring are from the twisted sector. They are more complicated to count but one checks that they yield the number of independent elements in $H^{1,1}(M)$. It is also straightforward to identify the orbifold action, like e.g. 6.56, 6.65), that leads to the mirrors $W$ of the manifolds $M$ in 4.24 in the conformal field theory context and to check that it indeed exchanges the $(c, c)$ with $(c, a)$ ring [69, 56]. A fascinating idea has been to use Cardy states [128] to classify D-branes as boundary conditions in the rational CFT at the Gepner-point and compare with geometric pictures of D-branes [18] in particular the triangulated category of coherent sheaves over $M$ for the $B$-branes or the category of special Lagrangian submanifolds of $M$ for the $A$-branes respectively.

\section{$4.5 t t^{*}$ equations, special geometry and contact terms}

The $t t^{*}$ equations describe the geometry of the ground states of $N=(2,2)$ two dimensional theories. The construction does not require necessarily conformal invariance, but rather the following structure. A nilpotent operator $Q$ and its adjoint $Q^{\dagger}$

$$
\left\{Q, Q^{\dagger}\right\}=H
$$

and a conserved fermion number. $Q$ and its adjoint $Q^{\dagger}$ define rings of cohomological operators $\mathscr{R}$ and $\mathscr{R}^{*}$ respectively. To make contact with the previous sections this can be realized as

$$
Q=\left\{\begin{array}{ll}
Q_{A}=Q_{-}+\bar{Q}_{+}, & \mathscr{R}=(a, c) \\
Q_{B}=\bar{Q}_{-}+\bar{Q}_{+}, & \mathscr{R}=(c, c)
\end{array} \quad Q^{\dagger}= \begin{cases}Q_{A}^{\dagger}=\bar{Q}_{-}+Q_{+}, & \mathscr{R}^{*}=(c, a) \\
Q_{B}^{\dagger}=Q_{-}+Q_{+}, & \mathscr{R}^{*}=(a, a)\end{cases}\right.
$$

As explained we have to twist the theories by identifying the corresponding $A^{R}$ gauge connection with the spin connection. Since only the fermion number must be conserved [31] one needs only a $Z_{2}$ anomaly free subgroup of the $U(1)_{R}$-currents. The $t t^{*}$ geometry is applicable to $N=(2,2)$ $2 \mathrm{~d}$ field theories with marginal (conformal) but also relevant (non-conformal) deformations. While these theories might not have a geometrical target space realization, it is still ${ }^{16}$ useful to think of a

\footnotetext{
${ }^{15}$ In general there might be $(c, c)$ states in the twisted sectors but for the smooth hypersurfaces, such as the quintic, there are none.

${ }^{16}$ For $\sigma$ model on $M$ this formal correspondence becomes an actual correspondence.
} 
formal correspondence to the deRham (Dolbeault) cohomology on a manifold $M$ with $\left(Q, Q^{\dagger}, H\right) \sim$ $\left(\mathrm{d}, \mathrm{d}^{*}, \Delta\right)$

The Ramond-Ramond vacuum states, compare (4.33), are defined by

$$
Q|\alpha\rangle=Q^{\dagger}|\alpha\rangle=0 \text {. }
$$

Such states play the rôle of harmonic forms. We call the space of vacua $\mathscr{H}$. The operator state correspondence of 2d QFT associates to every operator $\phi \in \mathscr{R}$ acting on a any vacuum state $\alpha$ a state $|\phi\rangle=\phi|\alpha\rangle$. In order to avoid too many indices we call the zero-form operators $\mathscr{O}^{(0)}=\phi$ and the two form operators $\mathscr{O}^{(2)}=\mathscr{O}$. Since $|\phi\rangle_{\alpha}=\phi|\alpha\rangle$ is closed, Hodge decomposition (7.24) applies $|\phi\rangle_{\alpha}=\left|\phi_{0}\right\rangle_{\alpha}+Q\left|\phi_{-}\right\rangle_{\alpha}+Q^{\dagger}\left|\phi_{+}\right\rangle_{\alpha}$ and by that we get a map

$$
\Pi_{h}:|\phi\rangle_{\alpha} \mapsto\left|\phi_{0}\right\rangle_{\alpha}
$$

from $\mathscr{R}$ to $\mathscr{H}$. If $\alpha$ is fixed and as will soon see there is preferred choice we can find a canonical map from the ring $\mathscr{R}$ to the Ramond-Ramond groundstates. Moreover every $\phi \in \mathscr{R}$ induces a map

$$
\Phi:|\alpha\rangle \mapsto\left|\phi_{0}\right\rangle_{\alpha}
$$

from $\mathscr{H}$ to $\mathscr{H}$. Everything we said from Eq. (4.43) on, could have been said verbatim for the conjugated sector defined by $Q^{\dagger}$. In particular we get for the same choice of $\alpha$ a second basis of $\mathscr{H}$, which we call $|\bar{\imath}\rangle, \bar{j}=1, \ldots, r$. If one has unbroken $U(1)_{R / L}$ symmetries as in Sec. 4.4 one could single out $|\alpha\rangle$ as the lowest charge state in the Ramond-Ramond groundstate.

The following path integral argument requires only conserved fermion number. In the operator approach[3] [40] to $2 \mathrm{~d}$ field theory one defines a state of the Hilbert space $H$ of $2 \mathrm{~d}$ theory by the path integral over a half sphere $H S^{2}$ bounding an $S^{1}$. Parametrize the $S^{1}$ by $\theta$ and denote the fields generically by $\phi(\theta)$. The path integral is a functional of the boundary field configuration $\phi(\theta) \in L^{2}$ on the $S^{1}$ and defines a state $|\phi\rangle$ in $H$ as in (4.47). Anti periodic boundary conditions for fermionic states on contractible loops as $S^{1}$ on $H S^{2}$ are the natural boundary conditions in the path integral so that (4.47) does not yield periodic Ramond-Ramond states in $H$. However the connection $A_{\mu}^{R}$ of the gauged $U(1)$ R-symmetry couples to the fermion number with charge $\frac{1}{2}$, i.e. acts like a spin connection $\omega_{\mu}$. When one transports the fermion along the $S^{1}$, the connection is integrated to a Wilson loop phase rotation acting on the fermionic state as

$$
e^{\pi i \oint_{S^{1}} \omega \mathrm{d} x}=e^{\pi i \int_{H S^{2}} \mathrm{~d} \omega}=e^{\pi i \int_{H S^{2}} \frac{R}{2 \pi i} \sqrt{h}}=e^{\pi i \int_{H S^{2}} c_{1}(T)}=-1,
$$

which rectifies the periodicity. ${ }^{17}$ A projection to the Ramond-Ramond groundstates at the boundary can now be achieved by attaching a cylinder of length $T$ to $H S^{2}$, see Fig. 3. Call the combined surface $H_{T} S^{2}$. The "evolution" of a state $|\phi\rangle$ defined by the original boundary $S^{1}$ of $H S^{2}$ to the far boundary is described by $e^{-H T}|\phi\rangle$. If the length $T$ of the cylinder goes to infinity only the groundstates in $\mathscr{H}$ survive, because they have 0 as energy eigenvalue of $H$, cff (4.34).

After this preparation we can define the path integral version of a projector (4.44)

$$
|i\rangle=\lim _{T \rightarrow \infty} \int \mathscr{D} \phi e^{-\int_{H_{T} S^{2}} L(\phi)} \phi_{i}=\Pi_{p}\left(\phi_{i}\right) .
$$

\footnotetext{
${ }^{17} \mathrm{~A}$ discussion of the axial anomalies of $2 \mathrm{~d} U(1)$ gauge theories can be found in Chap 19.1 of [124].
} 
$|0\rangle$

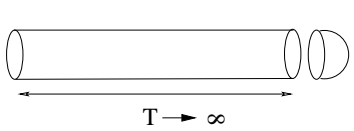

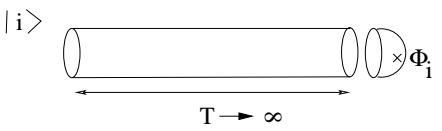

Figure 3: Path integral projectors to the Ramond-Ramond ground states $\mathscr{H}$

The $T \rightarrow \infty$ limit makes the projector only sensitive to cohomological information of ring states $\phi \in \mathscr{R}$ or $\bar{\phi} \in \mathscr{R}^{*}$. Exact pieces have non-zero energy and are completely suppressed. Note that $\Pi(\mathbf{1})=|0\rangle$ defines a preferred vacuum state. We call the image of a basis $\phi_{i} \in \mathscr{R}, i=0, \ldots, r$ with $\Phi_{0}=\mathbf{1}$ in $\mathscr{H}$ the topological basis $|i\rangle=\Pi_{p}\left(\phi_{i}\right)$. By the operator state correspondence we can also represent the rings (4.10) on the vacuum states

$$
\phi_{i}|j\rangle=C_{j k}^{k}|k\rangle
$$

The path integral (4.47) with insertions of $\bar{\phi}_{i} \in \mathscr{R}^{*}$ defines the anti-topological basis $|\bar{\imath}\rangle=$ $\Pi_{p}\left(\bar{\phi}_{i}\right)$. The two basis of $\mathscr{H}$ namely $|i\rangle$ and $|\bar{\imath}\rangle$ must be related by a linear transformation, the real structure,

$$
|i\rangle=M_{i}^{\bar{l}}|\bar{l}\rangle .
$$

The CPT theorem of the $2 \mathrm{~d}$ field theory states that the effect of complex conjugating all expressions in (4.47) sends $|i\rangle \rightarrow|\bar{\imath}\rangle$, i.e. $|\bar{\imath}\rangle=M|\dot{q}| j\rangle$ which implies $M M^{*}=1$. One has a topological bilinear pairing

$$
\langle i \mid j\rangle=\eta_{i j}
$$

and an hermitian bilinear pairing called the $t t^{*}$ metric

$$
\langle\bar{\imath} \mid j\rangle=g_{j},
$$

which are in an obvious way related by the real structure

$$
g^{\bar{l} i} \eta_{i j}=M_{j}^{\bar{l}}
$$

Note that $\langle i| \neq(|i\rangle)^{\dagger}$. Both bilinear pairings can be defined by the path integral as in Fig. 4 . These

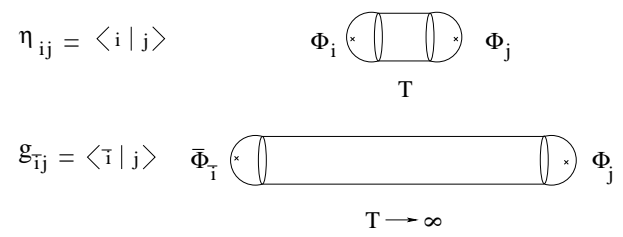

Figure 4: Path integral representation of the topological pairing $\eta_{i j}$ and the topological-antitopological pairing $g_{\bar{l}}$.

objects are topological to different extent. Changing the representative of the $Q$ cohomology class $|i\rangle \mapsto|i\rangle+Q|\lambda\rangle$ or $\langle j| \mapsto\langle j|+\langle\lambda| Q$ will do nothing in $\langle i \mid j\rangle$ as $|j\rangle$ and $\langle i|$ are Q closed. Due to (4.9) the pairing $\eta_{i j}$ is independent of the position. That is true for all length/diameter ratios of the cylinder, i.e. the cylinder is not needed at all in the definition. For the pairing $g_{\bar{\imath} j}$ with 
$\langle\bar{\imath}| \mapsto\langle\bar{\imath}|+\langle\lambda| \dot{Q}$ and $|i\rangle \mapsto|i\rangle+Q|\lambda\rangle$ the argument does not apply as $|j\rangle$ is not $Q^{\dagger}$ and $\langle\bar{\imath}|$ not $Q$ closed. However from (4.41) and $Q|\lambda\rangle \neq 0\left(\langle\lambda| Q^{\dagger} \neq 0\right)$ follows that these exact states have positive energy. The only states with zero energy are R-R vacua. I.e. in the case of $g_{\bar{i}, j}$ we need the $T \rightarrow \infty$ limit to define a topological quantity.

Locally the tangent space of the $\left(t, t^{*}\right)$ moduli space is spanned by elements from $\mathscr{R}(t)$ and $\mathscr{R}^{*}\left(t^{*}\right)$. It is clear that the pairing $\eta_{i j}$ depends only on the $t$ moduli. Moreover one shows that as metric it is completely flat, i.e. all components of the curvature tensor vanish similar as in $d<1$ strings [42]. One can therefore find coordinates which make the metric $\eta_{i j}$ constant. This defines the moduli dependent basis of $\mathscr{R}$. As it is clear from the construction of the basis $|i\rangle$ and $|\bar{\imath}\rangle$ via the projection of moduli dependent elements in the rings $\mathscr{R}$ and $\mathscr{R}^{*}$ they will depend on the moduli $\underline{m}=\left(\underline{t}, \underline{t}^{*}\right)$. In the Landau-Ginzburg approach [143] $\eta_{i j}$ is explicitly defined in terms of the Landau Ginzburg superpotential as

$$
\eta_{i j}=\operatorname{Res}\left[\phi_{i} \phi_{j}\right]=\frac{1}{(2 \pi i)^{n}} \int_{\Gamma} \frac{\phi(X) \mathrm{d} X^{1} \wedge \ldots \wedge \mathrm{d} X^{n}}{\partial_{1} W \ldots \partial_{n} W}=\sum_{\mathrm{d} W} \phi(X) \operatorname{det}^{-1}\left[\partial_{i} \partial_{j} W\right] .
$$

Another approach to define $\eta_{i j}$ is via the supersymmetric Schroedinger equation [29]. We will not dwell deeper into the derivation of (4.53), except for remarking that it is a zero dimensional analog of the Griffith residuum expressions (6.57,6.71) used in Sec. 6.7 to define the periods, with the identification $W=P$.

The $t t^{*}$ equations describe how the vacuum states in $\mathscr{H}$ vary over the moduli space parametrized by $\underline{m}$. One calls the corresponding bundle also $\mathscr{H}$. Let $e_{\gamma}$ be a basis, i.e. a section in $\mathscr{H}$, and denote its connection

$$
A_{\beta \gamma}^{\alpha}=g^{\alpha \kappa}\left\langle e_{\kappa}\left|\partial_{\beta}\right| e_{\gamma}\right\rangle
$$

If the basis of $\mathscr{H}$ changes by a "gauge" transformation $\left|e_{\gamma}\right\rangle \mapsto\left|e_{\gamma}^{\prime}\right\rangle=\Lambda_{\gamma \delta}\left|e_{\delta}\right\rangle$ then the connection undergoes a gauge transformation $A \mapsto \Lambda^{-1} A \Lambda+\Lambda^{-1} \mathrm{~d} \Lambda$. Let us consider the perturbation

$$
S=\int_{\Sigma} \mathrm{d}^{2} z \mathscr{L}_{0}+\sum_{i} t^{i} \int_{\Sigma} \mathrm{d}^{2} z \mathscr{O}_{i}+\sum_{\bar{l}} \bar{t}^{\bar{l}} \int_{\Sigma} \mathrm{d}^{2} z \overline{\mathscr{O}}_{i}
$$

where the two-form descendants are called $\mathscr{O}_{i}:=\mathscr{O}_{i}^{(2)}$. It is easy to show that the following mixed indices of this connection vanish in the holomorphic basis. Consider e.g. $A_{\bar{l} j}^{i}$ using 4.52 we can write $A_{\bar{l} j}^{i}=g^{i \bar{k}}\left\langle\bar{k}\left|\partial_{\bar{l}}\right| j\right\rangle=\eta^{i k}\left\langle k\left|\partial_{\bar{\imath}}\right| j\right\rangle$. By (4.12) we can write $\int_{\Sigma} \overline{\mathscr{O}}_{\bar{\imath}}=[Q, \Lambda]$ and since $\phi_{j}$ is $Q$ closed we can write $\partial_{\bar{\imath}}|j\rangle=\Pi_{h}\left([Q, \Lambda] \phi_{j}\right)=Q \Pi_{h}\left(\Lambda \phi_{j}\right)=Q(\Lambda|j\rangle)$. Since $\langle k| Q=0$ is closed this expression vanishes

$$
A_{\bar{l} j}^{i}=0 .
$$

Similarly one shows that $A_{k \bar{j}}^{i}=\eta^{i l}\left\langle l\left|\partial_{k}\right| \bar{j}\right\rangle=0$.

The metric connection is characterized by

$$
0=D_{k} g_{i \bar{j}}=\partial_{k} g_{i \bar{j}}-\left(\partial_{k}\langle i|\right)|\bar{j}\rangle-\left\langle i\left|\partial_{\bar{k}}\right| \bar{j}\right\rangle=\left(\partial_{k}\langle i|\right)|\bar{j}\rangle .
$$

From this and the $\bar{D}_{\bar{k}}$ derivative, we get formulas for $A_{k m}^{j}$ and $A_{\bar{k} \bar{m}}^{\bar{j}}$

$$
A_{k m}^{j}=g^{j \bar{j}} \partial_{k} g_{m \bar{j}}, \quad A_{\bar{k} \bar{m}}^{\bar{j}}=g^{m \bar{j}} \partial_{\bar{k}} g_{m \bar{m}}
$$


as hermitian connection of $g$. Indeed the topological basis $|i\rangle$ and the anti-topological basis $|\bar{\imath}\rangle$ form holomorphic and antiholomorphic sections of the vacuum bundle over the moduli $\underline{m}$ and one gets the vanishing of the following components of the curvature

$$
\left[D_{i}, D_{j}\right]=\left[\bar{D}_{\bar{i}}, \bar{D}_{\bar{j}}\right]=0 .
$$

The most important relation comes from analyzing the $\left[D_{i}, \bar{D}_{\bar{i}}\right]$ curvature term. Let us do this for definiteness for the $B$ model. Since the twisting (4.29) is so that $\bar{Q}_{+}(z) \sim G^{+}(z)$ and $\bar{Q}_{-}(z) \sim \bar{G}^{+}(z)$ have dimension one, we can define

$$
\bar{Q}_{+}=\oint \mathrm{d} z G^{+}(z), \quad \bar{Q}_{-}=\oint \mathrm{d} z \bar{G}^{+}(z) .
$$

Here we adopt the notation to use the CFT conventions for the twisted currents. The commutators and anticommutators in the definition of the descendants (4.12) can be represented by (4.26) as

$$
\begin{aligned}
& \mathscr{O}_{i}:=\mathscr{O}_{i}^{(2)}=\left\{Q_{+},\left[Q_{-}, \phi_{i}(u)\right]\right\} \sim \oint_{C_{u}} \mathrm{~d} z G^{-}(z) \oint_{C_{u}^{\prime}} \mathrm{d} w \bar{G}^{-}(w) \bar{\phi}_{\bar{l}}(u), \\
& \overline{\mathscr{O}}_{\bar{l}}:=\overline{\mathscr{O}}_{\bar{l}}^{(2)}=\left\{\bar{Q}_{+},\left[\bar{Q}_{-}, \bar{\phi}_{\bar{l}}(u)\right]\right\} \sim \oint_{C_{u}} \mathrm{~d} z G^{+}(z) \oint_{C_{u}^{\prime}} \mathrm{d} w \bar{G}^{+}(w) \bar{\phi}_{\bar{l}}(u)
\end{aligned}
$$

We calculate $\left[D_{i}, \bar{D}_{\bar{l}}\right]$ in $|l\rangle$ basis i.e.

$$
\begin{aligned}
{\left[D_{i}, \bar{D}_{\bar{j}}\right]_{k}^{l}=} & \partial_{i} A_{\bar{j} k}^{l}-\partial_{\bar{j}} A_{i k}^{l}=\eta^{l p}\left[\left(\partial_{i}\langle p|\right) \bar{\partial}_{\bar{j}}|k\rangle-\left(\bar{\partial}_{\bar{j}}\langle p|\right) \partial_{i}|k\rangle\right] \\
= & \eta^{l p} \Pi\left(\phi_{p} \int_{H S_{L}^{2}}\left\{Q_{+},\left[Q_{-}, \phi_{i}\right]\right\}\right) \Pi\left(\int_{H S_{R}^{2}}\left\{\bar{Q}_{+},\left[\bar{Q}_{-}, \bar{\phi}_{\bar{j}}\right]\right\} \phi_{k}\right) \\
& -\eta^{l p} \Pi\left(\phi_{p} \int_{H S_{L}^{2}}\left\{\bar{Q}_{+},\left[\bar{Q}_{-}, \bar{\phi}_{\bar{j}}\right]\right\}\right) \Pi\left(\int_{H S_{R}^{2}}\left\{Q_{+},\left[Q_{-}, \phi_{i}\right]\right\} \phi_{k}\right) \\
= & \eta^{l p}\left[\Pi\left(\phi_{p} \int_{H S_{L}^{2}} \partial \bar{\partial} \phi_{i}\right) \Pi\left(\int_{H S_{R}^{2}} \bar{\phi}_{\bar{j}} \phi_{k}\right)-\Pi\left(\phi_{p} \int_{H S_{L}^{2}} \bar{\phi}_{\bar{j}}\right) \Pi\left(\left(\int_{H S_{R}^{2}} \partial \bar{\partial} \phi_{i}\right) \phi_{k}\right)\right] \\
= & \eta^{l p}\left[\Pi\left(\phi_{p} \int_{H S_{L}^{2}} \bar{\phi}_{\bar{j}}\right) \Pi\left(\int_{C_{R}}\left(\partial_{\tau_{2}} \phi_{i}\right) \phi_{k}\right)-\Pi\left(\phi_{p} \oint_{C_{L}} \partial_{\tau_{2}} \phi_{i}\right) \Pi\left(\left(\int_{H S_{R}^{2}} \bar{\phi}_{\bar{j}}\right) \phi_{k}\right)\right] \\
= & \eta^{l p}\left[\Pi\left(\phi_{p} \int_{H S_{L}^{2}} \bar{\phi}_{\bar{j}}\right) \Pi\left(\left(\oint_{\Gamma} H(z) \oint_{C_{R}} \phi_{i}\right) \phi_{k}\right)-\Pi\left(\phi_{p} \oint_{\Gamma} H(z) \int_{C_{L}} \phi_{i}\right) \Pi\left(\left(\int_{H S_{R}^{2}} \bar{\phi}_{\bar{j}}\right) \phi_{k}\right)\right]
\end{aligned}
$$

the contours of $G^{-}(z), \bar{G}^{-}(z) G^{+}(z), \bar{G}^{+}(z)$ are as in Fig. (5). Moreover we consider operators $\phi$ in the $(c, c)$ and $\bar{\phi}$ in the $(a, a)$ ring, e.g. $\phi$ is $\bar{Q}_{+}$and $\bar{Q}_{-}$closed. In the language of current algebras that means that the short distance expansion of $\phi(v)$ with $\bar{Q}_{+}(z) \sim G^{+}(z)$ and $\bar{Q}_{-}(w) \sim \bar{G}^{+}(z)$ has no pole and $\phi(v)$ can be ignored when deforming $\Gamma_{z}$ and $\Gamma_{w}$. The contours e.g. of the term in the third line can be deformed as in fig. 5 and the contours of $G^{-}(z), \bar{G}^{-}(z)$ encircling $G^{+}(z), \bar{G}^{+}(z)$ give the $L_{-1}$ and $\bar{L}_{-1}$ acting as $\partial$ and $\bar{\partial}$ derivatives on $\phi_{i}$ by (4.9). Similar manipulations apply to the term in the second line of (4.62). Applying Gauss's law in both terms gives the integral over the normal derivative $-\partial_{\tau_{2}}$. The minus sign is due to the orientation of $\tau_{2}$. The normal direction is "time" evolution by $H$, i.e. $\partial_{\tau_{2}}=\partial_{n} \phi_{i}=\left[H, \phi_{i}\right]$, which is used in the last line of 4.62, where $H(z)$ is integrated around $\phi_{i}$. From now on we exploit the topological nature of the theory and take ordered limits of $\Sigma$

$$
\text { first : } \quad T_{R}, T_{L} \rightarrow \infty, \quad \text { second : } \quad T \rightarrow \infty
$$

as depicted Fig.6. The tubes are all normalized to have perimeter 1. Elongation $T_{R}$ and $T_{L}$ projects 


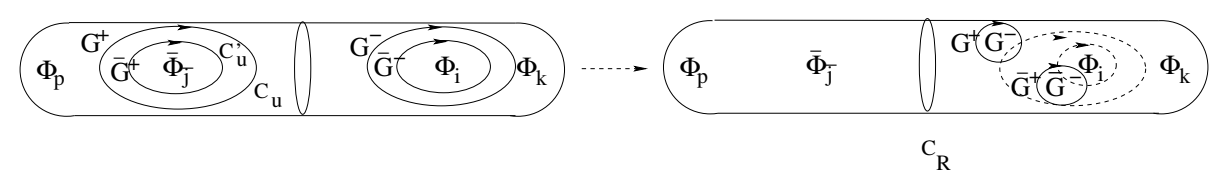

Figure 5: Contour manipulation on $\Sigma$ in the evaluation of $\left[D_{i}, \bar{D}_{\bar{j}}\right]_{k}^{l}$.

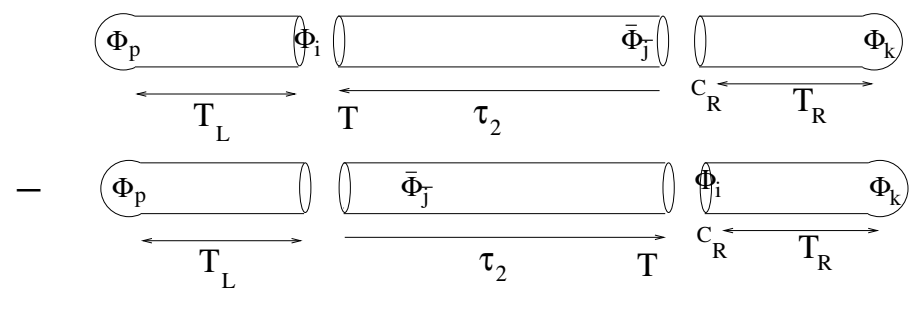

Figure 6: Limits taking in the evaluation of $\left[D_{i}, \bar{D}_{\bar{j}}\right]_{k}^{l}$.

$\phi_{p}$ and $\phi_{k}$ to the Ramond-Ramond vacuum state $\langle p|$ and $|k\rangle$ respectively. The procedure of the limits is a prescription how to deal with short distance singularities and the only such issue in topological field theory are contact terms see (4.71) and (5.32).

The action of $H$ on these states yields zero. The two terms in the last line of (4.62) are transformed into each other by exchanging the left- and right infinity. We discuss the $-\Pi\left(\phi_{p} \int_{H S_{L}^{2}} \bar{\phi}_{\bar{j}}\right) \times$ $\Pi\left(\left(\oint_{\Gamma} H(z) \oint_{C_{R}} \phi_{i}\right) \phi_{k}\right)$ explicitly. Vanishing of $H|k\rangle$ means that $H$ may considered as acting on the full state $\Pi\left(\left(\oint_{C_{R}} \phi_{i}\right) \phi_{k}\right)$. In Hilbert space notation it is denoted as $\left.H\left|\left(\oint_{C_{R}} \phi_{i}\right)\right| k\right\rangle$ and similarly $\Pi\left(\phi_{p} \int_{H S_{L}^{2}} \bar{\phi}_{\bar{j}}\right)$ as $\left\langle p\left|\int_{H S_{L}^{2}} \bar{\phi}_{\bar{j}}\right|\right.$. We can move the $H$ integral to the left and since $\phi_{p}$ is projected to the groundstate the non-vanishing contribution comes from its action on $\int_{H S_{L}^{2}} \bar{\phi}_{\bar{j}}$. If the insertion of $\bar{\phi}_{j}$ is on the leftmost part in fig (6) it will also be projected to the groundstate in the $T \rightarrow \infty$ limit and annihilated by $H$. Therefore it remains to consider the contribution from integral over the middle tubes whose length is parametrized by $T$. This integral is $\int_{T u} \bar{\phi}_{\bar{j}}=\int_{0}^{T} \mathrm{~d} \tau_{2} \oint_{C_{L}} \mathrm{~d} \theta \bar{\phi}_{\bar{j}} . H$ creates $\tau_{2}$ translations, so $\left[H, \bar{\phi}_{\bar{j}}\right]=-\partial_{\tau_{2}} \bar{\phi}_{\bar{j}}$ and the integration over $\tau_{2}$ becomes trivial. Note that only the lower boundary $\tau_{2}=0$ contributes. The upper boundaries, where $\bar{\phi}_{\bar{j}}$ is near $\phi_{i}$ in both contributions see Fig. 6, cancels. Therefore

$$
\begin{aligned}
{\left[D_{i}, \bar{D}_{\bar{j}}\right]_{k}^{l} } & =\eta^{l p} \lim _{T_{L / R} \rightarrow \infty}\left[\Pi\left(\phi_{p} \int_{H S_{L}^{2}} \bar{\phi}_{\bar{j}}\right) \Pi\left(\left(\oint_{\Gamma} H \oint_{C_{R}} \phi_{i}\right) \phi_{k}\right)-\Pi\left(\phi_{p} \oint_{\Gamma} H \int_{C_{L}} \phi_{i}\right) \Pi\left(\int_{H S_{R}^{2}} \bar{\phi}_{\bar{j}} \phi_{k}\right)\right] \\
& =\eta^{l p}\left[\left\langle p\left|\left(\int_{T u} \bar{\phi}_{\bar{j}}\right) H\left(\oint_{C_{R}} \phi_{i}\right)\right| k\right\rangle-\left\langle p\left|\left(\int_{C_{L}} \phi_{i}\right) H\left(\int_{T u} \bar{\phi}_{\bar{j}}\right)\right| k\right\rangle\right] \\
& =\eta^{l p} \lim _{T \rightarrow \infty}\left[\left\langle p\left|\left(\oint_{C_{L}} \bar{\phi}_{\bar{j}}\right) e^{-H T}\left(\oint_{C_{R}} \phi_{i}\right)\right| k\right\rangle-\left\langle p\left|\left(\int_{C_{L}}\right) \phi_{i} e^{-H T}\left(\oint_{C_{R}} \bar{\phi}_{\bar{j}}\right)\right| k\right\rangle\right] \\
& =\left(\bar{C}_{\bar{j}} C_{i}\right)_{k}^{l}-\left(C_{i} \bar{C}_{\bar{j}}\right)_{k}^{l}=-\left[C_{i}, \bar{C}_{\bar{j}}\right]_{k}^{l}
\end{aligned}
$$

This is the main identity within the $t t^{*}$ equations. The others are easier to derive and all are summarized below in the topological basis

$$
\begin{gathered}
{\left[D_{i}, \bar{D}_{\bar{j}}\right]=-\left[C_{i}, \bar{C}_{\bar{j}}\right]} \\
{\left[D_{i}, D_{j}\right]=\left[\bar{D}_{\bar{l}}, \bar{D}_{\bar{j}}\right]=\left[D_{i}, \bar{C}_{\bar{j}}\right]=\left[\bar{D}_{\bar{l}}, C_{j}\right]=0} \\
D_{i} C_{j}=D_{j} C_{i} \quad \bar{D}_{\bar{l}} \bar{C}_{\bar{j}}=\bar{D}_{\bar{j}} \bar{C}_{\bar{l}}
\end{gathered}
$$


We can now define a flat $\left[\nabla_{i}, \nabla_{j}\right]=\left[\nabla_{i}, \bar{\nabla}_{\bar{j}}\right]=\left[\bar{\nabla}_{\bar{i}}, \bar{\nabla}_{\bar{j}}\right]=0$ connection

$$
\nabla_{i}=D_{i}+\alpha C_{i}, \quad \bar{\nabla}_{\bar{j}}=\bar{D}_{\bar{j}}+\alpha^{-1} \bar{C}_{\bar{j}}
$$

The sections of the vacuum bundle are identified with the periods in the Calabi-Yau $\sigma$ model context. The above flat connection goes by the name Gauss Manin connection. In this context, see Sec. 6.5. Since it is flat it seems that the theory is trivial! However flat connections can still have monodromies, over non simply connected manifolds, see Fig. 16,17, which are the essential data of our theories. Where do these monodromies come from? The key is that (4.23), which is based on a local consideration of the tangent spaces of metric deformations at a generic point of the moduli space fails at singular degenerations of the space time Calabi-Yau manifold. At these loci charged Ramond-Ramond states become light, the simplest example is the charged black hole at the conifold [134], which sits in a hyper multiplet. In the presence of massless charged states the supergravity argument for the factorization (4.23) into hyper- and vector multiplets does not apply either. In fact the logarithm in third period that produces the monodromy $M_{1}$ in (6.75) can be interpreted as the one loop correction of the vector multiplet gauge coupling due to the massless hypermultiplet.

The $t t^{*}$ equations describe the essence of the WS super symmetry constraints on the topological correlators. These equations have in general to be supplemented with information about the structure constants $C_{i j}^{l}$ and boundary conditions. But already with some $U(1)$ i.e. $R$ symmetry charge constraints they become powerful. E.g. for $d<1$ (4.37) implies $|Q|<1$ moreover these theories are rational and have finitely many chiral primaries in this charge range. We assign to the $t^{i}$ of say the $(c, c)$ ring (4.55) the weight $w_{i}=\left(1-Q_{i}\right)>0$. The last equation (4.65) called associativity guarantees the existence of a potential $\mathscr{F}$ with $C_{i j k}=D_{i} D_{j} D_{k} \mathscr{F}$. As discussed one can chose flat coordinates, which we call for convenience also $t^{i}$ such that $C_{i j k}=\partial_{i} \partial_{j} \partial_{k} \mathscr{F}$ Charge conservation implies that $\mathscr{F}$ is homogeneous of degree 2 in the weights $w_{i}$ of the $t^{i}$, i.e. a finite polynomial and associativity determines its coefficients up to an overall normalization. These constraints imply indeed that there is a completely solvable discrete infinite set of $d<1 N=(2,2)$ theories with an $A D E$ classification. For $d \geq 1$ there are zero and negative weight $t^{i}$ and this simple way of approaching the problem loses its grip.

However if $d \in \mathbf{Z}$ and the $R$ charges are also integer, we expect from Sec. 4.4 that beside WS super symmetry also ST supersymmetry constrains the correlators. Let us show that (4.65) implies for the Calabi-Yau $\sigma$ models on threefolds $d=3$ and odd integer R charges special Kähler geometry. In the holomorphic basis we use 4.56) to write $\left[D_{i}, \bar{D}_{\bar{j}}\right]_{l}^{k}=-\bar{\partial}_{\bar{j}} A_{i l}^{k}=-\left[C_{i}, \bar{C}_{\bar{j}}\right]$. With $\left(C_{i l}^{k}\right)^{\dagger}=\bar{C}_{\bar{i} \bar{k}}^{\bar{l}}$ and hence $C_{\bar{j} m}^{k}=g^{k \bar{k}} C_{\bar{j} k}^{\bar{m}} g_{\bar{m} m}$ we write

$$
\bar{\partial}_{\bar{j}} A_{i l}^{k}=\left[C_{i}, \bar{C}_{\bar{j}}\right]_{l}^{k}=\left[C_{i}, g^{-1} C_{j}^{\dagger} g\right]_{l}^{k} .
$$

In the case of Calabi-Yau $\sigma$ model the $R$ charge conservation law forbids many correlators, see sections 5.1 and 6.1. In particular $g_{0 \bar{k}}=g^{0 \bar{k}}=0$ for $\bar{k} \neq \overline{0}$ and $C_{i 0}^{k}=\delta_{i}^{k}$ and $C_{\overline{0} \overline{0}}^{\bar{k}}=\delta_{\bar{l}}^{\bar{k}}$. If we 
specialize (4.67) to $k=l=0$ we can write

$$
\begin{aligned}
\bar{\partial}_{\bar{j}} A_{i 0}^{0}=\bar{\partial}_{\bar{j}}\left(g^{0 \bar{k}} \partial_{i} g_{0, \bar{k}}\right) & =\left[C_{i}, g^{-1}\left(C_{j}\right)^{\dagger} g\right]_{0}^{0} \\
\bar{\partial}_{\bar{j}} \partial_{i} \log \left(g_{0 \overline{0}}\right) & =-g^{0 \overline{0}} C_{\overline{\bar{j}} \bar{k}} g_{\bar{k} i} \\
& =-\frac{g_{\bar{j} i}}{g_{0 \overline{0}}} .
\end{aligned}
$$

As follows from the identification (6.5, 6.6) in the B-model and (6.23) or Serre duality (7.54) the vacuum states $|0\rangle$ and $|\overline{0}\rangle$ are associated to the holomorphic $(n, 0)$ and anti-hololomorhic $(0, n)$ forms. In particular

$$
e^{-K}=i \int_{M} \Omega \wedge \bar{\Omega}=\langle\overline{0} \mid 0\rangle
$$

and comparing (6.47, 6.48) with (4.68, 4.69) we identify the Weil-Peterson metric with a sub-block of the $t t^{*}$ metric

$$
G_{i \bar{j}}=g_{i \bar{j}} e^{K}
$$

In (4.64) we have related the curvature of $g_{i \bar{j}}$ to a bilinear in the 3-point functions and with 4.70) this becomes the special geometry relation (6.53). In other words $t t^{*}$ in genus 0 implies special Kähler geometry, but the main virtue of the formalism is that it generalizes readily special Kähler geometry to higher genus. This will become essential to solve the $B$-model.

It is worth mentioning the closely related contact term approach to the definition of the connection 4.57, see e.g. 104 for a short introduction. It does use conformal invariance and restricts the analysis to exactly marginal ring operators. If the operators are exactly marginal for all values of $t=\{t, \bar{t}\}$ of marginal perturbation parameters as (4.55) then the most general short distance expansion in the basis $e_{\gamma}$ of them is

$$
\mathscr{O}_{\alpha}(z) \mathscr{O}_{\beta}(0) \sim \frac{G_{\alpha \beta}}{|z|^{4}}+\Gamma_{\alpha \beta}^{\gamma} \delta^{2}(z) \mathscr{O}_{\gamma}(0)
$$

Clearly this expansion is compatible with dimensional analysis, $\delta^{2}(z)=\frac{\partial}{\partial z} \frac{1}{\bar{z}}$. Marginality implies in first order in $t$ that $\int \mathrm{d}^{2} z\left\langle\mathscr{O}_{\alpha}(z) \mathscr{O}_{\beta}(1) \mathscr{O}_{\gamma}(0)\right\rangle$ gets only contributions from $z=1$ and $z=0$, which explains that only the $\delta$-function appears on the right of (4.71) in this order. Exact marginality means that scale independence, i.e. vanishing $\beta$ functions, are maintained to all orders in $t$. To next order follows the closing on exactly marginal operators, as opposed to arbitrary $(1,1)$ operators, on the right in (4.71). The Zamolodchikov metric is defined as the sphere correlator

$$
G_{\alpha \beta}=\left\langle\mathscr{O}_{\alpha}(1) \mathscr{O}_{\beta}(0)\right\rangle
$$

and because of conformal invariance it does not require a limit as in the $t t^{*}$ case. Taking the derivatives with respect to perturbations one gets

$$
\frac{\partial G_{\alpha \beta}}{\partial t_{\gamma}}=\int \mathrm{d}^{2} z\left\langle\mathscr{O}_{\alpha}(z) \mathscr{O}_{\beta}(1) \mathscr{O}_{\beta}(0)\right\rangle=\Gamma_{\alpha \gamma}^{\delta} G_{\delta \beta}+\Gamma_{\gamma \beta}^{\delta} G_{\delta \alpha},
$$

which establishes $\Gamma_{\alpha \gamma}^{\delta}$ as connection of the Zamolodchikov metric. So far the discussion of the contact terms has been about a general ansatz and in particular all $\Gamma_{\alpha \gamma}^{\delta}$ could have been zero. 
However [68] observed first that in order to ensure marginality in superconformal theories with non trivial triple couplings $C_{i k}^{k}$ the contact terms have to be present, which is of course required to get (4.65). The virtue of the $t t *$ equations is to generalize this analysis to all ring states replacing $\Gamma_{\alpha \beta}^{\delta}$ with $A_{\alpha \beta}^{\delta}$ and non-conformal theories.

As an exercise one may derive the special geometry relation in $N=(2,2)$ SCFT using the contact term approach as a specialization of the derivation of the $t t^{*}$ equations. The decomposition of $\alpha, \beta$ into $j \bar{j}$ comes from the possibility of picking the holomorphic basis in $N=(2,2)$ WS theories. Of course the real challenge is to understand the occurrence of the monodromies, which we identified as the data of the theory, which however requires to understand the spacetime Ramond-Ramond states.

\section{The topological $A$-model}

As mentioned above the gauged $U(1)_{V}$ symmetry becomes not anomalous and this topological model can be defined on any Kähler manifold.

\subsection{A model without worldsheet gravity}

In this section we want to describe the operators and correlation functions of topological $A$ topological and their relation to the geometry of the target space $M$. We call the anticommuting scalars from table $2 \chi^{i}:=\psi_{-}^{i}$ and $\chi^{\bar{i}}:=\bar{\psi}_{+}^{\bar{i}}$ and the one forms i.e. sections of $K$ and $\bar{K}$ are denoted by $\rho_{z}^{\bar{l}}=\bar{\psi}_{-}^{\bar{l}}$ and $\rho_{\bar{z}}:=\psi_{+}^{i}$. The action is then

$$
L=2 t \int \mathrm{d}^{2} z\left(g_{i \bar{j}} \partial_{v} x^{i} \partial^{v} x^{\bar{j}}+i \varepsilon^{\mu v} b_{i \bar{j}} \partial_{\mu} x^{i} \partial_{v} x^{\bar{j}}-i g_{i j} \rho_{z}^{\bar{j}} D_{\bar{z}} \chi^{i}+i g_{i j} \rho_{\bar{z}}^{i} D_{z} \chi^{\bar{j}}-\frac{1}{2} R_{i \bar{k} j \bar{l}} \rho_{\bar{z}}^{i} \chi^{j} \rho_{z}^{\bar{k}} \chi^{\bar{l}}\right)
$$

where we added the term involving the antisymmetric 2 -form $b_{i \bar{j}} \in H_{2}(M, Z)$, which plays an important rôle in the bosonic sector of the topological $A$ model. Supersymmetry $\delta=\bar{\varepsilon}_{-} \bar{Q}_{+}+\varepsilon_{+} Q_{-}$ acts by

$$
\begin{aligned}
\delta x^{i} & =\varepsilon_{+} \chi^{i}, & \delta x^{\bar{l}} & =\bar{\varepsilon}_{-} \chi^{\bar{\imath}} \\
\delta \rho_{\bar{z}}^{i} & =2 i \bar{\varepsilon}_{-} \partial_{\bar{z}} x^{i}+\varepsilon_{+} \Gamma_{j k}^{i} \rho_{\bar{z}}^{j} \chi^{k}, & \partial \chi^{\bar{\imath}} & =0 \\
\delta \chi^{i} & =0, & \delta \rho_{z}^{\bar{l}} & =-2 i \bar{\varepsilon}_{+} \partial_{z} x^{\bar{l}}+\bar{\varepsilon}_{-} \Gamma_{\bar{j} k}^{\bar{l}} \rho_{z}^{\bar{k}} \chi^{\bar{j}}
\end{aligned}
$$

with $\delta^{2}=0$. There is a fixpoint of $\delta$ on fermionic zero mode configuration with $x^{i}$ a holomorphic map $x: \Sigma_{g} \rightarrow M$, i.e. $\partial_{z} \bar{x}^{\bar{j}}=\partial_{\bar{z}} x^{i}=0$, on which the path integral will localize by the fermionic zero mode integration, so that the bosonic integration reduces to an integration over the moduli space $\mathscr{M}$ of such holomorphic maps ${ }^{18}$. This moduli space will be labeled by the following topological data: the genus of $g$ and the homology class $\left[x_{*}\left(\Sigma_{g}\right)\right] \in H_{2}(M, Z)$ of the image of $\Sigma_{g}$ in $M$. The 0 -form correlation observables are combinations of $x^{i}, x^{\bar{l}}$ and $\chi^{i}, \chi^{\bar{i}}$ the latter anticommutating operators can be identified with the forms on $M$, i.e $\chi^{i} \leftrightarrow \mathrm{d} x^{i}$ and $\chi^{\bar{i}} \leftrightarrow \mathrm{d} x^{\bar{l}}$ One checks that under this correspondence $Q_{-}$and $\bar{Q}_{+}$are identified with the exterior derivatives of Dolbault cohomology $\partial$ and $\bar{\partial}$. Since then $Q=Q_{-}+\bar{Q}_{+}$is identified with the deRham operator $\mathrm{d}=\partial+\bar{\partial}$ one can

\footnotetext{
${ }^{18}$ In considering only $Q_{A}=\bar{Q}_{+}+Q_{-}$, i.e. setting $\varepsilon_{+}=\bar{\varepsilon}_{-}$one neglects structure, which would give information about the individual cohomology groups of $\mathscr{M}$.
} 
summarize the correspondence between the BRST cohomology of $Q_{A}$ and the deRham cohomology of $M$ as follows. For each form

$$
W=w_{I_{1}, \ldots, I_{n}}(x) \mathrm{d} x^{I_{1}} \wedge \ldots \wedge \mathrm{d} x^{I_{n}}
$$

on $M$ there is a topological operator

$$
\mathscr{O}_{W(P)}^{(0)}=w_{I_{1}, \ldots, I_{n}}(x) \chi^{I_{1}} \ldots \chi^{I_{n}}(P)
$$

of the A-model and the operation of $Q_{A}$ is identified with the exterior derivative

$$
\left\{Q_{A}, \mathscr{O}_{W}\right\}=-\mathscr{O}_{\mathrm{d} W}
$$

where the form degree $n$ of $W$ is identified with the ghost number of $\mathscr{O}_{W}$, since $\chi$ has ghost number +1 .

The action can be written as

$$
S=i t \int_{\Sigma} \mathrm{d}^{2} z\{Q, V\}+t \int_{\Sigma} x^{*}(\omega), \quad \text { with } \quad V=g_{i \bar{j}}\left(\rho_{z}^{\bar{\imath}} \partial_{\bar{z}} x^{j}+\partial_{z} x^{\bar{i}} \rho_{\bar{z}}^{j}\right)
$$

and

$$
\int_{\Sigma} x^{*}(\omega)=\int_{\Sigma} \mathrm{d}^{2} z\left(\partial_{z} x^{i} \partial_{\bar{z}} x^{\bar{j}} g_{i \bar{j}}-\partial_{\bar{z}} x^{i} \partial_{z} x^{\bar{j}} g_{i \bar{j}}\right)=\omega \cdot \beta \geq 0,
$$

where $\omega$ is the Kähler form $\omega=-i g_{i j} \mathrm{~d} z^{i} \mathrm{~d} z^{\bar{j}}$ and $\beta$ is the cohomology class $\left[x_{*}(\Sigma)\right]$ of the image of $\Sigma$ and the positivity holds if $\omega$ is in the Kählercone. If the antisymmetric tensor field $B$ is non-zero we replace $\omega$ by a complexified Kähler form $\omega_{c}=\omega+i B=i\left(b_{i \bar{j}}-i g_{i \bar{j}}\right) \mathrm{d} z^{i} \mathrm{~d} z^{\bar{j}}$.

The correlation function of the physical operators

$$
\left\langle\prod_{i=1}^{n} \mathscr{O}_{i}\right\rangle_{\beta}=e^{-i t \beta \cdot \omega} \int_{\mathscr{M}_{\beta}} \mathscr{D} x \mathscr{D} \chi \mathscr{D} \rho e^{-i t\left\{Q, \int V\right\}} \prod_{i=1}^{n} \mathscr{O}_{i}
$$

depends on the metric of $M$ only via the Kähler class $\omega$ (or on the complexified Kählerclass $\omega_{C}$ ). Other metric dependence in particular on the complex structure of $M$ as well as on $\Sigma_{g}$ appears in $V$. However this dependencies appears only as a $Q$ exact expression in (5.8) and decouples by (4.8). Moreover taking the derivative w.r.t. $t$ implies by (4.8) that the second factor is independent of $t$ and the correlation can be calculated for $\omega$ in the Kählercone for Ret $>0$ in limit of infinite $t$ i.e. at the classical minimum of the action. If we write

$$
\begin{aligned}
S_{B} & =\int_{\Sigma} g_{i \bar{j}}\left(\partial_{z} x^{i} \partial_{\bar{z}} x^{\bar{j}}+\partial_{\bar{z}} x^{i} \partial_{z} x^{\bar{j}}\right) \\
& =2 \int_{\Sigma} g_{i \bar{j}} \partial_{\bar{z}} x^{i} \partial_{z} x^{\bar{j}}+\int_{\Sigma} x^{*}(\omega)
\end{aligned}
$$

it is obvious that this minimum is taken at holomorphic maps $\partial_{\bar{z}} x^{i}=\partial_{z} x^{\bar{j}}=0$, which is another way to understand the supersymmetric localization. As mentioned the path integral collapses by this mechanism to a finite dimensional integrals over an in general infinite series of components of moduli spaces of holomorphic maps which are labeled by $\mathscr{M}_{g, \beta}(M)$.

Let us now discuss the selection rules for correlators $\left\langle\prod_{k=1}^{n} \mathscr{O}_{W_{k}}\right\rangle_{\beta}$. We note from table 2 and the identification of $\chi^{i}$ and $\chi^{\bar{i}}$ that $\chi^{i}$ has charge $q_{l}=-1$ and $q_{r}=0$ under the left and right $U(1)_{l / r}$ respectively, while $\chi^{\bar{\imath}}$ has $q_{l}=0$ and $q_{r}=1$. Because of the splitting of the tangent bundle 
of $M=T^{(1,0)} \oplus T^{(0,1)}$ we can associate to $\mathscr{O}_{W_{k}}$ an element in the Dolbeault cohomology group $H^{\left(p_{k}, q_{k}\right)}$. Since the vector $U(1)_{V}$ is unbroken in the quantum theory we get a charge conservation constraint $q_{V}=\sum_{k=1}^{n} p_{k}-\sum_{k=1}^{n} q_{k}=0$ for the correlator to respect vector charge conservation. For the classical axial charge we would get naively $q_{A}=\sum_{k=1}^{n} p_{k}+\sum_{k=1}^{n} q_{k}=0$. However the $U(1)_{A}$ is anomalous. Its anomaly is given by the index of the Dolbault operator and can be calculated by the Hirzebruch-Riemann-Roch theorem, see Sec. 7.3

$$
\begin{aligned}
q_{A} & =\#(\chi \text { zero modes })-\#(\rho \text { zero modes })=2\left(h^{0}\left(x^{*}(T M)\right)-h^{1}\left(x^{*}(T M)\right)\right) \\
& =2 \int_{\Sigma} \operatorname{ch}\left(x^{*}\left(T M^{(1,0)}\right)\right) \operatorname{td}(T \Sigma)=2\left(c_{1}(T M) \cdot \beta+\operatorname{dim}_{C} M(1-g)\right) .
\end{aligned}
$$

Combining the constraints we get

$$
\sum_{k=1}^{n} q_{k}=\sum_{k=1}^{n} p_{k}=c_{1}(T M) \cdot \beta+\operatorname{dim}_{C} M(1-g) .
$$

In particular for $g=0$ we can have a non-vanishing coupling $\left\langle\mathscr{O}_{W_{i}} \mathscr{O}_{W_{j}} \mathscr{O}_{W_{k}}\right\rangle$, where all $W_{l}$ are $(1,1)$ forms.

With two non-degenerate pairings we can associate a divisor $D_{k} \in H_{4}(M)$ to each $W_{(1,1)}^{(k)}$. One has $\int_{M} W_{(1,1)}^{(k)} \wedge W_{(l)(2,2)}=\delta_{l}^{k}$ as well as $\int_{D_{i}} W_{(j)(2,2)}=\delta_{j}^{i}$. If $\beta$ denotes the cohomology class of the image $C$ of the worldsheet in $M$ then we can write the product $\beta \cdot \omega=2 \pi \sum_{k=1}^{h^{1,1}} t_{k} d_{k}$, where $d_{k}=C \cap D_{k}$ is the number of intersections of $C$ with $D_{k}$ or the degree of $C$ w.r.t. $D_{k}$. The map with $d_{k}=0$ for all $k$ is special. It maps the three punctured sphere $\sigma_{0,3}$ to a point in $M$. One always find a representative of $w_{(1,1)}^{(k)}$ that has $\delta$-function support on $D_{k}$. This implies that the point in $\mathscr{O}_{w^{k}}\left(P_{k}\right)$ maps to $D_{k}$. If $\Sigma_{0,3}$ maps to a point in $M$ the path integral collapses hence to the intersection number of $D_{i} \cap D_{j} \cap D_{k}$. We define $q_{k}=e^{-2 \pi i t_{k}}$ then the correlation function ${ }^{19}$ is

$$
C_{i j k}(t)=\left\langle\mathscr{O}_{W_{i}} \mathscr{O}_{W_{j}} \mathscr{O}_{W_{k}}\right\rangle=D_{i} \cap D_{j} \cap D_{k}+\sum_{\left\{d_{i}\right\} \neq\{0\}} r_{\left\{d_{i}\right\}}^{g=0}\left(D_{i}, D_{j}, D_{k}\right) \prod_{i=1}^{h^{1,1}} q_{i}^{d_{i}}
$$

This deformed intersection is piece of the structure known as quantum cohomology ring of $M$. It is a deformation of the classical cohomology ring on $M$ by the parameters $q_{k}$. One needs in general the deformations of all pairings $[m]: H^{\otimes n} \rightarrow H$ indexed by $m \in H^{*}\left(\mathscr{M}_{0, n+1}\right)$, see [111] and [32] for a review, which we can be provided on the mirror side. Note that the relation to classical intersections in the limit picks a natural normalization of the operators $\mathscr{O}_{W}$ and of their two-point functions.

One collective effect of the instantons corrections is that structure functions $C_{i j k}(t)$ behaves smoothly at singularities in codimension two in $M$ as for instance through flop transitions [162][5].

We note from table 2 and 3 and from (4.12) that the $U(1)_{V}$ as well as the $U(1)_{A}$ charge of the operator $\mathscr{O}_{W_{j}}^{(2)}$ vanishes. In view of (4.18) this means that non-vanishing derivatives of $C_{j k l}(t)$ such as

$$
\left.\frac{\partial}{\partial t^{i}}\left\langle\mathscr{O}_{w^{j}} \mathscr{O}_{w^{k}} \mathscr{O}_{w^{l}}\right\rangle\right|_{t^{i}=0}=\left\langle\mathscr{O}_{w^{j}} \mathscr{O}_{w^{k}} \mathscr{O}_{w^{l}} \int_{\Sigma} \mathscr{O}_{w^{i}}^{(2)}\right\rangle
$$

\footnotetext{
${ }^{19}$ We abbreviate $\prod_{i=1}^{h^{1,1}} q_{i}^{d_{i}}=q^{\beta}$ in the following.
} 

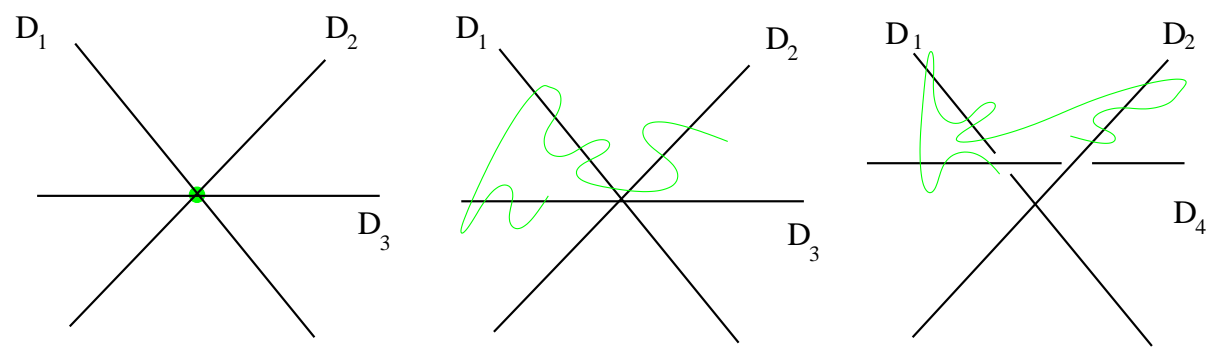

Figure 7: This figure shows instanton corrections to the coupling $C_{123}$ with $D_{1} \cap D_{2} \cap D_{3}=O(1)$ and $C_{124}$ with $D_{1} \cap D_{2} \cap D_{4}=0$. From the left to the right we pictured an instanton of degree 0 contributing of $O(1)$ to $C_{123}$, an instanton of degree $d_{1}=5, d_{2}=3, d_{3}=4$ contributing $\sim q_{1}^{5} q_{2}^{3} q_{3}^{4}$ to $C_{123}$ and an instanton of degree $d_{1}=5, d_{2}=4, d_{4}=3$ contributing $\sim q_{1}^{5} q_{2}^{4} q_{4}^{3}$ to $C_{124}$. Roughly speaking for large radii second the coupling $C_{124}$ is expected to be exponentially supressed against the first $C_{123}$. The precise statement depends on the growth of $r_{\left\{d_{i}\right\}}^{g=0}\left(D_{i}, D_{j}, D_{k}\right)$. Such collective effects of the intantons can be analyzed best in the $B$-model.

do exist according to the selection rules. This non-vanishing correlators signal that a non-trivial deformation family exist, but do not contain new information once $c_{j k l}(t)$ is known as function after summing up all intantons or easier from a B-model calculation. By $S L(2, \mathbf{C})$ invariance on $S^{2}$ there is a symmetry between fixing any three of the $\{i, j, k, l\}$ points and integrating over the fourth. This implies that

$$
\partial_{i} C_{j k l}(t)=\partial_{j} C_{i k l}(t)
$$

which is the integrability condition for the existence of a function $\mathscr{F}^{(0)}(t)$ with the property that

$$
C_{i j k}(\underline{t})=\partial_{i} \partial_{j} \partial_{k} \mathscr{F}^{(0)}(t)
$$

where we defined $\partial_{i}=\frac{\partial}{\partial t^{i}}$. This is in perfect accordance with facts concerning $\mathscr{F}(t)$ from the analysis of the vector moduli space of $N=2$ supergravity in 4 d, which is identified in type IIA compactifications with complexified Kähler moduli space. This facts can also be established in the complex structure deformation space, see Sec. (6.5), which again is identified by mirror symmetry with the complexified Kähler moduli space of the $A$-model. We should finally note that eqs. $5.13-5.15$ are not written covariantly, but rather in special coordinates. Covariant derivatives are discussed in the B-model section.

\subsection{Coupling the A model to worldsheet gravity}

While we have prepared our topological theories by the twist to make sense on any genus Riemann surface, we have ignored the degrees of freedom of the worldsheet metric in our discussion so far. As explained in Sec. 4.2 in string perturbation theory one has to integrate over the complex structure of the worldsheet and the position of the insertion points, in other words over the moduli space of Riemann surfaces with $n$ insertion of operators $\mathscr{M}_{g, n}$. We have rightfully ignored that in the genus zero correlator (5.12), because fixing three points kills the $S L(2, \mathbf{C})$ invariance of $S^{2}$ which has no complex structure deformations, so that $\mathscr{M}_{0,3}=$ point. Despite the fact that (5.11) predicts a nontrivial zero point function for $g=1$, without integrating over the complex structure of $\Sigma$ the answer for the correlation function $\mathscr{F}^{(1)}$ would be generically vanishing. As an intuitive examples consider maps from $\Sigma=T^{2}$ to $M=T^{2}$, allowed by the selection rule (5.11). If we fix the 
complex structure of $\Sigma$ and $M$ there would be, by definition of inequivalent complex structures, no holomorphic maps unless we hit with the complex structure parameter $\tau_{\Sigma}$ the one of $\tau_{M}$. Including all multicoverings [10] the answer $\mathscr{F}^{(1)}=-\log \left(\eta\left(\tau_{M}\right)\right) \delta\left(\tau_{M}-\tau_{\Sigma}\right)$ begs to be integrated over $\tau_{\Sigma}$ as it is natural in string theory as explained below. For higher genus (5.11) predicts vanishing of the correlation functions. That means if we fix the worldsheet metric there are just no holomorphic maps from a genus $g>1$ Riemann surface to $M$.

\subsection{Topological gravity}

The simplest example of string theory where integration over the the moduli space discussed in Sec. 4.2 is required is pure topological gravity. This is an good warm up example in which $M$ is replaced by a point. It plays a pivotal rôle for the $A$ - as well as for the $B$-model coupling to gravity. The calculation of the expected dimension (7.42) was for smooth curves, which represent an open top dimensional subset of the moduli space of all curves. In order to integrate of $\mathscr{M}_{g}$ we need some compactification of $\mathscr{M}_{g}$. Including nodal curves, but so that the the automorphism group, which is finite for smooth curves of $g>1$, stays finite is called the stable Deligne-Mumford compactification $\overline{\mathscr{M}}_{g}$. Genus zero curves have a $\operatorname{SL}(2, \mathbf{C})$ automorphism and $g=1$ curves an $z \rightarrow z+c$ automorphism. These can be killed either by the position of a node or a puncture. Because of the latter fact it is convenient to extend the discussion right away to punctured Riemann surfaces. Inserting a so called puncture operator 1 at the point $x \in \Sigma$ in the path integral means that we want to restrict the diffeomorphism group in (4.15) to a subgroup which preserves that point $x$. We call the moduli space with $n$ punctures $\mathscr{M}_{g, n}$. Its dimension is enhanced by $n$ complex dimensions relative to $\mathscr{M}_{g}$. Intuitively one may picture the movement of the point as additional dimension of $\mathscr{M}_{g, n}$. The more accurate picture is complementary. The restriction of the diffeomorphism group by the part, which moves the point in the denominator of (4.15) enhances the dimension.

Let us call punctures and ordinary double points (nodes) special points of $\Sigma$. The DeligneMumford compactification $\overline{\mathscr{M}}_{g, n}$ is the appropriate compactification to define good measures on $\overline{\mathscr{M}_{g, n}}$ in topological string theory. [151, 145]. It allows the above special points under the condition that they do not meet. The further conditions that

- (i) every irreducible component of genus 0 has at least three special points

- (ii) every irreducible component of genus 1 has at least one special point

guarantee that there are no continuous automorphism groups acting on $\overline{\mathscr{M}}_{g, n}$. Finite automorphism groups Aut are like gauge symmetries which are divided out. The resulting orbifold is the connected, irreducible, compact, non-singular Deligne Mumford stack of dimension $3 g-3+n$, denoted also by $\overline{\mathscr{M}_{g, n}}$.

The positive dimension of this space appears as an anomalous negative ghost number violation in the BRST quantization. In topological gravity it is compensated by insertion of descendant fields $\sigma_{n}(x)$ whose form degree is counted as positive ghost number. These descendant fields are constructed geometrically as the first Chern class of the complex line bundle $L_{i}=x_{i}^{*}(\omega)$ over $\overline{\mathscr{M}}_{g, n}$ in the universal curve $\mathscr{C} \overline{M_{g, n}}$, which is induced from the restriction of the holomorphic cotangent bundle $\left.T^{*} \Sigma_{g}\right|_{x_{i}}$ of $\Sigma_{g}$ to $x_{i}$. The universal curve is the fibration over $\overline{\mathscr{M}}_{g, n}$ whose fibers are the Riemann surfaces with $n$ punctures described by the point $\left[\Sigma, x_{1}, \ldots, x_{n}\right] \in \overline{\mathscr{M}}_{g, n} . \omega=\mathscr{K}_{\mathscr{C}} / \mathscr{M}$ is 


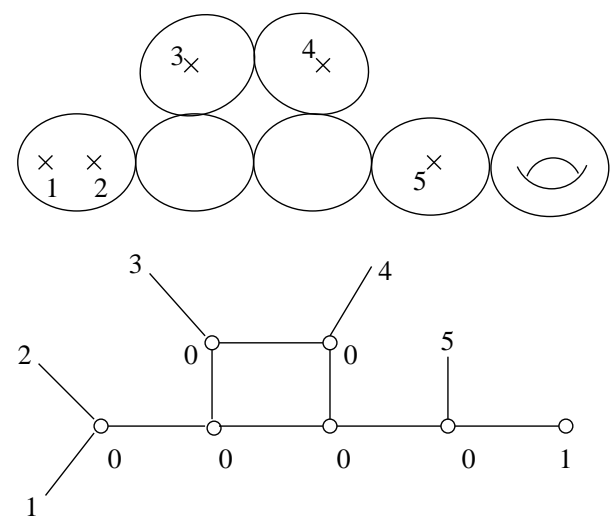

Figure 8: This figure shows a stable degeneration of a genus 2 curve with 5 marked points in $\overline{\mathscr{M}_{2,5}}$ as actual configuration above and as dual graph below.

roughly the cotangent bundle along the fibres. More precisely since nodal singularities are allowed it is the corresponding relative dualizing sheaf. $L_{i}$ are line bundles over $\overline{\mathscr{M}}_{g, n}$, see Fig. 9 .

The first Chern class $\psi_{i}=c_{1}\left(L_{i}\right)$ might be represented by the $(1,1)$ curvature form (7.33)

$$
\psi_{i}=-\frac{i}{\pi} \partial \bar{\partial} \log \left|\sigma_{x_{i}}\right|^{2}
$$

on $\mathscr{M}_{g, n}$, where $\sigma_{x_{i}}$ is a meromorphic section of $L_{i}$. It can be wedged to define the descent operators $\sigma_{n}\left(x_{i}\right):=\psi_{i}^{n}$ of form degree or ghost number $2 n$. We can also consider the insertion of $\sigma_{0}(x)=$ $\psi^{0}(x)$, the above mentioned puncture operator. What this means is that we change the moduli $\mathscr{M}_{g}$ to one $\mathscr{M}_{g, 1}$ in which the diffeomorphism group in (4.15) is restricted to fix one point without doing anything else. The selection rule for a non vanishing correlator

$$
\left\langle\sigma_{d_{1}} \ldots \sigma_{d_{n}}\right\rangle=\int_{\mathscr{M}_{g, r}} \psi_{1}^{d_{1}} \wedge \ldots \wedge \psi_{n}^{d_{n}}
$$

is now given simply by counting form degrees of insertions against the dimension of $\mathscr{M}_{g, n}$, which yields the condition [151, 145]

$$
\sum_{i=1}^{n}\left(d_{i}-1\right)=3 g-3
$$

Two easy and universal properties of the correlators (5.17), called topological recursion relations [149], are the puncture equation, referred also to as the string equation

$$
\left\langle\sigma_{0} \sigma_{d_{1}} \ldots \sigma_{d_{n}}\right\rangle=\sum_{d_{i} \neq 0}\left\langle\sigma_{d_{1}} \ldots \sigma_{d_{i}-1} \ldots \sigma_{d_{n}}\right\rangle
$$

and the dilaton equation [149]

$$
\left\langle\sigma_{1} \sigma_{d_{1}} \ldots \sigma_{d_{n}}\right\rangle=(2 g-2+n)\left\langle\sigma_{d_{1}} \ldots \sigma_{d_{n}}\right\rangle .
$$

Let us give the original arguments [149] that lead to (5.19, 5.20). The argument can be made mathematically rigorous see [77]. In equations both a puncture is removed from the left relative to the right side and the nontrivial relation comes from loci in $\overline{\mathscr{M}_{g, n+1}}$, where this removed point 
$x_{0}$ is together with exactly one other $x_{j}$ in a genus zero component $S_{j}^{2}$ of the degenerate curve (the bold fibre in Fig. 9), so that its removal destabilizes $\overline{\mathscr{M}}_{g, n}$. We will discuss the generic case and leave the special $g=0, n=2$ and $g=1, n=1$ situations to the reader. The key point is now that $L_{i}=x_{i}^{*}(\omega)$ over $\overline{\mathscr{M}}_{g, n+1}$ and $L_{i}^{\prime}=x_{i}^{*}\left(\omega^{\prime}\right)$ over $\overline{\mathscr{M}}_{g, n}, i=1, \ldots, n$ are related in a non trivial way. If it would be the case that $L_{i}=\pi^{*}\left(L_{i}^{\prime}\right)$ then starting with the right hand side we could argue that the left hand side in (5.19, 5.20) vanishes due to (5.18).

These relevant issues occur at the divisors $D_{j}$ in $\overline{\mathscr{M}}_{g, n+1}$ (in Fig. ? we show just $D_{1}$ ). The forgetful map $\pi: \overline{\mathscr{M}}_{g, n+1} \rightarrow \overline{\mathscr{M}}_{g, n}$ is a fibering map, whose fiber describes the position of the point $x_{0}$, which is essentially $\Sigma$. Its lift to the universal curves $\pi_{\mathscr{C}}: \mathscr{C} \overline{\mathscr{M}}_{g, n+1} \rightarrow \mathscr{C} \overline{\mathscr{M}}_{g, n}$ not a fibering as $\pi_{\mathscr{C}}$ also contracts the unstable $S_{j}^{2}$. There is however an isomorphism $\alpha$ between $\alpha: \overline{\mathscr{M}}_{g, n+1} \cong \mathscr{C} \overline{\mathscr{M}}_{g, n}$. It is just not compatible with the fibering $\pi: \overline{\mathscr{M}}_{g, n+1} \rightarrow \overline{\mathscr{M}}_{g, n}$. Now if $s$ is a section of $\omega^{\prime}$ then the evaluation $x_{j}^{*}(s)$ at $x_{j}$ pulls back under $\pi^{*}$ to section $\pi^{*} x_{j}^{*}(s)$ of $\omega$ over $\overline{\mathscr{M}_{g, n+1}}$. A simple local model near the contracted $S_{j}^{2}$ shows that $\pi^{*} x_{j}^{*}(s)$ vanished with order one at $D_{j}$. This implies $L_{j}=\pi^{*}\left(L_{j}\right) \otimes \mathscr{O}\left(D_{j}\right)$ with $\psi_{j}=c_{1}\left(L_{j}\right)$ and the properties about characteristic classes summarized in Sec. (7.3) one gets

$$
\psi_{j}=\psi_{j}^{*}+\left[D_{j}\right]
$$

The algebraic identity

$$
\psi_{j}^{n}=\left(\psi_{j}^{*}\right)^{n}+\left[D_{j}\right] \sum_{k=1}^{n-1} \psi_{j}^{k}\left(\psi_{j}^{*}\right)^{n-k-1}
$$

simplifies to $\psi_{j}^{n}=\left(\psi_{j}^{*}\right)^{n}+\left[D_{j}\right]\left(\psi_{j}^{*}\right)^{n-1}$ as $\psi_{j}=c_{1}\left(L_{j}\right)\left[D_{j}\right]=0$, because $L_{j}$ is trivial over $D_{j}$ as the sphere $S_{2}^{j}$ with its three special points is rigid.

So we can evaluate

$$
\begin{aligned}
\left\langle\sigma_{0} \sigma_{d_{1}} \ldots \sigma_{d_{n}}\right\rangle & =\int_{\overline{\mathscr{M}}_{g, n+1}} \mathbf{1} \wedge \wedge_{i=1}^{n} \psi_{i}^{d_{i}}=\sum_{j=1}^{n} \int_{\overline{\mathscr{M}}_{g, n+1}}\left[D_{j}\right] \wedge_{i=1}^{n}\left(\psi_{i}^{*}\right)^{d_{i}-\delta_{i j}} \\
& =\sum_{j=1}^{n} \int_{\overline{\mathscr{M}}_{g, n}} \psi_{1}^{d_{1}} \wedge \ldots \psi_{j}^{d_{j}-1} \ldots \wedge \psi_{1}^{d_{1}}=\sum_{j=1}^{n}\left\langle\sigma_{d_{1}} \ldots \sigma_{d_{j}-1} \ldots \sigma_{d_{n}}\right\rangle
\end{aligned}
$$

Here we used $\left[D_{i}\right] \cdot\left[D_{j}\right]=0$ which follows from the definition and in the third equality we have integrated over the fiber of $\pi: \overline{\mathscr{M}}_{g, n+1}$ where $\left[D_{j}\right]$ represents a section with a simple zero. Very similarly one concludes that $L_{0}=\alpha^{*}\left(\omega^{\prime}\right) \otimes_{j=1}^{n} \mathscr{O}\left(D_{j}\right)$ is a degree $2 g-g+n$ section of a line bundle over the fibre of $\pi$. We evaluate then again by integration over the fibre

$$
\left\langle\sigma_{1} \sigma_{d_{1}} \ldots \sigma_{d_{n}}\right\rangle=\int_{\overline{\mathscr{M}}_{g, n+1}} \psi_{0} \wedge_{i=1}^{n} \psi_{i}^{d_{i}}=(2 g-2+n)\left\langle\sigma_{d_{1}} \ldots \sigma_{d_{n}}\right\rangle
$$

With the recursive relations (5.19,5.20) and the initial conditions that the moduli space of a three pointed sphere is a point $\left\langle\sigma_{0} \sigma_{0} \sigma_{0}\right\rangle=1$ and $\left\langle\sigma_{1}\right\rangle$ one can solve as an exercise all $g=0,1$ correlators. It seems natural to try next to consider maps which "forget" nodes to get a recursion among correlations with different genera. From the algebraic point of view taken above this turns out to be surprisingly difficult.

Let now $\left\{d_{i}\right\}$ the set of all nonnegative integers and define

$$
F_{g}\left(t_{0}, t_{1}, \ldots\right)=\sum_{\left\{d_{i}\right\}}\left\langle\prod \tau_{d_{i}}\right\rangle_{g} \prod_{r>0} \frac{t_{r}^{n_{r}}}{n_{r} !},
$$




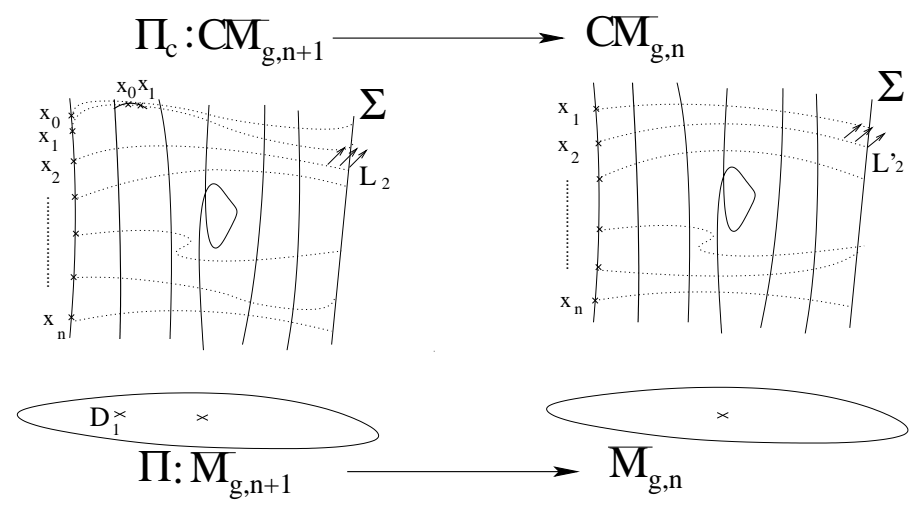

Figure 9: Universal Curve $\mathscr{C} M_{g, n+1}$ and the forgetful map. The nodal and reducible fibre are displayed, because there are such fibres, but they plays no rôle in the derivations of the string and dilaton equation. They would play a rôle in recursion relations among different genera, which is hard from the algebraic point op view.

with $n_{r}=\operatorname{Card}\left(i: d_{i}=r\right)$ and

$$
F=\sum_{g=0}^{\infty} \lambda^{2 g-2} F_{g},
$$

the free energy of $2 \mathrm{~d}$ topological gravity. Where we rescaled the operators $\tau_{n}=(2 n+1) ! ! \sigma_{n}$ for latter convenience. [149] conjectured that the partition function $Z=e^{F}$ satisfies the Virasoro constraints

$$
L_{n} Z=0, \quad n \geq-1 \quad \text { with }\left[L_{n}, L_{m}\right]=(n-m) L_{n+m}
$$

with

$$
\begin{aligned}
L_{-1} & =-\frac{1}{2} \frac{\partial}{\partial t_{0}}+\frac{1}{4} t_{0}^{2}+\sum_{i=1}^{\infty} \frac{2 i+1}{2} t_{i} \frac{\partial}{\partial t_{i-1}} \\
L_{0} & =-\frac{1}{2} \frac{\partial}{\partial t_{1}}+\sum_{i=0}^{\infty} \frac{2 i+1}{2} \frac{\partial}{\partial t_{i}}+\frac{1}{16} \\
L_{n} & =-\frac{1}{2} \frac{\partial}{\partial t_{n-1}}+\sum_{i=0}^{\infty} \frac{2 i+1}{2} t_{i} \frac{\partial}{\partial t_{i+n}}+\frac{\lambda^{2}}{4} \sum_{i=0}^{n} \frac{\partial^{2}}{\partial t_{i-1} \partial t_{n-i}}
\end{aligned}
$$

As an exercises one may check that $5.19,5.20$ ) are equivalent to $L_{-1} Z=L_{0} Z=0$. It is well known [149] [41] that (5.27) is equivalent to the fact that $Z$ is the $\tau$ function of the KDV hierachy and fulfills the dilaton equation.

All proofs of (5.27) are combinatorial. The first is by Kontsevich, who interprets a direct evaluation of the correlators as ribbon graphs of the shifted Airy function matrix model, which in turn can by viewed as the Akhiezer Baker function of the KdV hierarchy. This beautiful work [99] has been reviewed in many places e.g. [40, 39]. More recently a second combinatorial proof has been given by Okounkov and Pandharipande [122]. Recently a new proof has been given by Mirzakhani [115], which establishes an interesting relation to the Weil-Peterson volume of the moduli space of hyperbolic Riemann surfaces with geodesic boundary conditions that awaits physical interpretation.

There is a physical recursion argument relation based on the contact term algebra of two dimensional gravity [145], which up to a normalization of $\left\langle\tau_{0} \tau_{0} \tau_{0}\right\rangle=1$ reproduces all correlation 


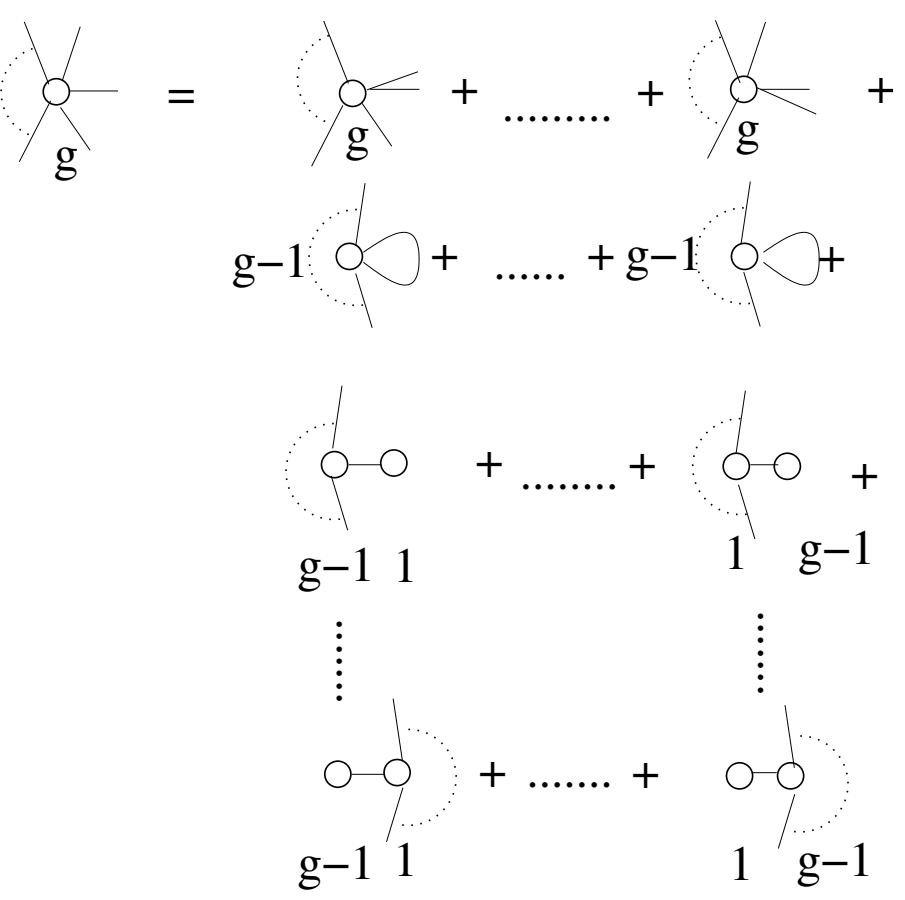

Figure 10: Degenerations of a genus $g$ surface corresponding to the codim one boundary in $\overline{\mathscr{M}}_{g, n}$ in the dual graph notations where closed lines are double points and open lines are operator insertions.

functions and is equivalent to 5.27, see [40]. The recursion includes a reduction of the genus

$$
\begin{aligned}
\left\langle\tau_{n} \prod_{k \in S} \tau_{k}\right\rangle_{g}= & \sum_{k \in S} P_{k}^{(n)}\left\langle\tau_{n+k-1} \prod_{k^{\prime} \neq k} \tau_{k^{\prime}}\right\rangle_{g}+\sum_{i+j=n-2} A_{i j}^{(n)}\left\langle\tau_{i} \tau_{j} \prod_{k \in S} \tau_{k}\right\rangle_{g-1} \\
& +\sum_{h=1}^{g-1} \sum_{\substack{S=S_{1} \cup S_{2} \\
i+j=n-2}} B_{i j}^{(n, h)}\left\langle\tau_{i} \prod_{k \in S_{1}} \tau_{k}\right\rangle_{h}\left\langle\tau_{i} \prod_{k \in S_{2}} \tau_{k}\right\rangle_{g-h}
\end{aligned}
$$

This recursion reads very naturally as if we could have reduced in addition to the unstable meeting of two points also the nodes and irreducible fibres in Fig. 9 and treat all boundaries of the moduli space $\overline{\mathscr{M}}_{g, n}$ at the same footing as in Fig. 10. [145] determine the $P_{k}^{(n)}=2 k+1$ and $A_{i j}^{(n)}=\frac{1}{2}$ and $B_{i j}^{(n, h)}=\frac{1}{2}$ using contact term manipulations. The puncture and the dilaton equation, which is implied in 5.29) can be established rigorously in this way. However for the determination of all $A, B, P$ one needs consistency assumptions about the contact term algebra. Therefore, even though (5.29) implies (5.27), the approach of [145] is not a quite a proof of (5.27).

Let us sketch the argument [145] of the identification of the $2 \mathrm{~d}$ field theory formalism with the geometrical approach. $2 \mathrm{~d}$ gravity can be constructed as cohomological supersymmetric theory with two nilpotent operators $Q$ representing the total BRST charge and $Q_{-}=Q_{s}-\bar{Q}_{s}$, where $Q_{s}$ are the left and $\bar{Q}_{s}$ the right super charge. The decoupling of the WS metric is not complete $\left\{Q_{s}, \beta^{k}\right\}=\left\{Q, \beta^{k}\right\}=T^{k}$, so that $Q$ and $Q_{s}$ insertions in correlations act on the measure (6.96) and yield by (4.17) derivatives on $\mathscr{M}_{g, n}$. The decisive field is the 2 d dilaton $\phi$. Other fields have the following relation to $\phi$

$$
\begin{array}{rlrl}
\psi-\bar{\psi} & =\frac{1}{2}\left\{Q_{-}, \phi\right\}, & \gamma_{0} & =\frac{1}{2}\left\{Q,\left\{Q_{-}, \phi\right\}\right\} \\
(\omega, \bar{\omega}) & =\frac{1}{2}(\partial \phi,-\bar{\partial} \phi),\left(\psi_{0}, \bar{\psi}_{0}\right)=\frac{1}{2}(\partial \psi,-\bar{\partial} \psi), \quad R=\mathrm{d} \omega=\partial \bar{\partial} \omega .
\end{array}
$$




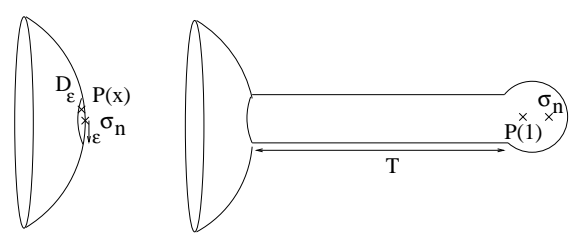

Figure 11: Conformally equivalent definition of colliding points.

The theory has a gauge fixing sector similar to the superstring and in particular anti-commutating $(b, c)$ ghost and commuting $(\beta, \gamma)$ ghosts with BRST symmetry $\delta_{b r s t} \omega=\phi_{0}+\mathrm{d} c_{0}, \delta_{b r s t} c_{0}=\gamma_{0}$, $\delta_{b r s t} \omega=\phi_{0}+\mathrm{d} c_{0}, \delta_{b r s t} \psi_{0}=\mathrm{d} \gamma_{0}$ and $\delta_{b r s t} \gamma_{0}=0$. The field equations imply $\gamma_{0}=\frac{1}{2}(\partial \gamma+\gamma \partial \phi+$ $c \partial \psi+$ c.c.). The main claim is that formally the $\psi_{i}$ classes are

$$
\psi_{i} \sim\left(\gamma_{0}+\psi_{0}+\mathrm{d} \omega\right)\left(x_{i}\right)
$$

so that formally $\sigma_{n} \sim\left(\gamma_{0}+\psi_{0}+\mathrm{d} w\right)^{n}$. The point is that the insertion of $\left\langle\left(\gamma_{0}+\psi_{0}+\mathrm{d} \omega\right) \mathscr{O}\right\rangle_{g}$ produces by (5.30) and (4.17) a two-form on $\overline{\mathscr{M}}_{g}$ namely $\partial \bar{\partial}\langle\phi \mathscr{O}\rangle_{g}$, where the $\partial \bar{\partial}$ operators act on $\overline{\mathscr{M}}_{g, n}$ and $\mathscr{O}$ stands for cohomological states. Note that the $\partial, \bar{\partial}$ derivatives act in the direction of the complex moduli by (4.17) as well as in the direction of the fibre of the universal curve. There are operators $\mathscr{O}_{i}=e^{\pi\left(x_{i}\right)}$, so that $\left\langle\phi \mathscr{O}_{i}\right\rangle_{g}=\log \left|\sigma_{x_{i}}\right|^{2}$ hence by $(5.16)$ we get the claimed relation. The puncture operator plays a special rôle in the field theory formalism and is given in the -1 picture [126] by $P(x)=c \bar{c} \delta(\gamma) \delta(\bar{\gamma})(x)$. In order to prove the puncture equation (5.19) one has to understand the contact term between $P$ and $\sigma_{n}$, that is the integral

$$
\begin{aligned}
\int_{D_{\varepsilon}} P\left|\sigma_{n}\right\rangle & =\int_{|x|<\varepsilon} \mathrm{d}^{2} x P(x)\left|\sigma_{n}\right\rangle=\int_{|q|<\varepsilon} \frac{\mathrm{d}^{2} q}{|q|^{2}} G_{0} \bar{G}_{0} q^{L_{0}} \bar{q}^{L_{0}} P(1)\left|\sigma_{n}\right\rangle \\
& =\int_{|q|<\varepsilon} \frac{\mathrm{d}^{2} q}{|q|^{2}} G_{0} \bar{G}_{0} q^{L_{0}} \bar{q}^{L_{0}} \frac{1}{2} Q Q_{-}\left|\sigma_{n-1}\right\rangle=\int_{|q|<\varepsilon} \mathrm{d}^{2} q \partial_{q} \bar{\partial}_{\bar{q}}\left(q^{L_{0}} \bar{q}^{L_{0}}\left|\sigma_{n-1}\right\rangle\right) \\
& =\int_{|q|<\varepsilon} \mathrm{d}^{2} q \partial_{q} \bar{\partial}_{\bar{q}}\left(\log |q|^{2}\right)\left|\sigma_{n-1}\right\rangle=\left|\sigma_{n-1}\right\rangle
\end{aligned}
$$

where we replaced in the second equality the position dependence of the puncture operator by a neck of length $T=-\log |q|$, see figure 11 . The insertion of the $G_{0}, \bar{G}_{0}$ comes from the integral over the superpartners of the modulus $q$. From the definition (5.31) and $\sigma_{n}=\psi^{n}$ as well as (5.30) follows the third equality. The $G_{0}, \bar{G}_{0}$ play the same rôle as the $Q_{+}, Q_{-}$in the derivation (4.64) namely to produce the derivatives $q \partial_{q} \bar{q} \bar{\partial}_{\bar{q}}$ from the anti commutator $\left\{Q, G_{0}\right\}=\left\{Q_{-}, G_{0}\right\}=L_{0}$. The logarithm occurs, because $\left[L_{0}, \phi(0)\right]=\phi_{0}+1$ with $\phi_{0}=\oint \partial \phi$ and $\phi_{0}\left|\sigma_{n-1}\right\rangle=L_{0}\left|\sigma_{n-1}\right\rangle=0$. Regular terms vanish under the integral. Hence one concludes that

$$
P(x)\left|\sigma_{n}\right\rangle=\delta^{(2)}(x)\left|\sigma_{n-1}\right\rangle
$$

from which (5.19) follows. The derivation of the dilaton equation is a very similar exercise. The rest of (5.29) is application of the sewing procedure of string perturbation theory with some consistency considerations restricting the contact algebra [145]. We will a make a similar construction in Sec. 6.12 
Beside the classes $\Psi$ there are other important classes on $\mathscr{M}_{g, n}$. A smooth Riemann surface $\Sigma_{g}$ has a $g$ dimensional vector space of holomorphic differentials in $H^{1,0}\left(\Sigma_{g}\right)=H^{0}\left(\Sigma_{g}, K_{\Sigma_{g}}\right)$. On a connected nodal curves there is extension of these differentials. Namely on a curve of arithmetic genus $g$ one has $g$ meromorphic differentials $\omega$, which are holomorphic outside the nodes, have at most a pole of order 1 at each node branch and the residua on the two node branches add up to zero. These vector space patch together to give a rank $g$ vector bundle $E$ on $\overline{\mathscr{M}}_{g, n}$, which is called the Hodge bundle ${ }^{20}$. In fact the construction applies likewise to $\overline{\mathscr{M}_{g, n}(M, \beta)}$, see below. The Chern classes of the Hodge bundle. sometimes referred to as $\lambda$ classes, can be integrated over $\overline{\mathscr{M}}_{g, n}$. For $g \geq 2$ one gets [52] [51]

$$
R_{g}=\int_{\overline{\mathscr{M}}_{g}} c_{g-1}^{3}(E)=\frac{\left|B_{2 g} B_{2 g-2}\right|}{2 g(2 g-2)(2 g-2) !}
$$

e.g. $R_{2}=\frac{1}{2880}, R_{3}=\frac{1}{725760}, \ldots$. Using a formula of Mumford the correlators involving $c_{k}(E)$ and $\psi$ can be systematically reduced [52] to correlators involving only $\psi$ and are fixed by the Virasoro constraints.

\subsection{The moduli space of maps}

Let us now come to the original question of coupling the topological $A$-model to gravity. We now want to construct the moduli space of maps which send $x: \Sigma \rightarrow M$ into a class $\beta=[x(\Sigma)] \in$ $H_{2}(M, \mathbf{Z})$ called $\mathscr{M}_{g, n}(M, \beta)$. The rough expectation is that the negative dimension of the moduli space (5.11) for $g>1$ is offset by the dimension of the deformations space $\mathscr{M}_{g}$ of the Riemann surface. In other words we might hope to modify the complex structure $j$ of $\Sigma$ until it is compatible with the complex structure on $M$ and a $(j, J)$ holomorphic map satisfying the Cauchy-Riemann equations

$$
\partial_{j, J} x=\frac{1}{2}(\mathrm{~d} x+J \circ \mathrm{d} x \circ j)=0
$$

does exist. To see at least heuristically what the dimension of the moduli space of a stable compactification $\overline{\mathscr{M}_{g, n}(M, \beta)}$ is consider the normal bundle exact sequence of an immersion of a non singular curve in $M$

$$
0 \rightarrow T_{\Sigma} \rightarrow x^{*} T_{M} \rightarrow N_{\Sigma / M} \rightarrow 0
$$

The associated long exact sequence is

$$
\begin{aligned}
0 & \rightarrow H^{0}\left(\Sigma, T_{\Sigma}\right) \rightarrow \\
H^{0}\left(\Sigma, x^{*} T_{M}\right) \rightarrow H^{0}\left(\Sigma, N_{\Sigma / M}\right) & \rightarrow H^{1}\left(\Sigma, T_{\Sigma}\right) \rightarrow \\
H^{1}\left(\Sigma, x^{*} T_{M}\right) \rightarrow H^{1}\left(\Sigma, N_{\Sigma / M}\right) & \rightarrow 0
\end{aligned}
$$

Let us interpret the terms as automorphism, deformations and obstructions of the involved structures. As far as only the domain curve is concerned we know that $H^{1}\left(\Sigma, T_{\Sigma}\right)-H^{0}\left(\Sigma, T_{\Sigma}\right)=$ $\operatorname{Def}(\Sigma)-\operatorname{Aut}(\Sigma)$, and that the dimension of $\mathscr{M}_{\}}$is $3 g-3$. For fixed complex structure of the domain we can identify $H^{0}\left(\Sigma, x^{*} T_{M}\right)$ with the deformations and $H^{1}\left(\Sigma, x^{*} T_{M}\right)$ with the obstructions of the map $x$. The real objects of interest are $H^{0}\left(\Sigma, N_{\Sigma / M}\right)$ and $H^{1}\left(\Sigma, N_{\Sigma / M}\right)$ which are identified with the deformations and obstructions of the map $x$ without fixing the domain. In order to have a

\footnotetext{
${ }^{20} \mathrm{~A}$ similar construction on the targetspace is discussed in Sec. 6.5 .
} 
stable compactification $\overline{\mathscr{M}_{g, n}(M, \beta)}$ we must allow in general for marked points. In this case (5.37) becomes

$$
\begin{aligned}
0 & \rightarrow \operatorname{Aut}(\Sigma, \underline{p}, x) \rightarrow \operatorname{Aut}(\Sigma, \underline{p}) \rightarrow \\
\operatorname{Def}(x) & \rightarrow \operatorname{Def}(\Sigma, \underline{p}, x) \rightarrow \operatorname{Def}(\Sigma, \underline{p}) \rightarrow \\
\operatorname{Obs}(x) & \rightarrow \operatorname{Obs}(\Sigma, \underline{p}, x) \rightarrow 0
\end{aligned}
$$

Now if a stable compactification $\overline{\mathscr{M}_{g, n}(M, \beta)}$ exist then $\operatorname{Aut}(\Sigma, \underline{p}, X)=0$. Moreover at least in some relevant situations $\operatorname{Obs}(\Sigma, \underline{p}, X)=0$ and since the alternating dimensions of long exact sequences is 0 , we can calculate $\operatorname{Def}(\Sigma, p, X)$, because we know $\operatorname{Def}(\Sigma, p)-\operatorname{Aut}(\Sigma, p)=3 g-3+n$ and $\operatorname{Def}(x)-\operatorname{Obs}(x)=h^{0}\left(x^{*}(T M)\right)-h^{1}\left(x^{*}(T M)\right)$. The expected or virtual complex dimension of the moduli of stable maps is

$$
\begin{aligned}
\operatorname{vdim}_{C} \overline{\mathscr{M}}_{g, \beta, n}(x) & =h^{0}\left(x^{*}(T M)\right)-h^{1}\left(x^{*}(T M)\right)+\operatorname{dim} \operatorname{Def}(\Sigma, \underline{p})-\operatorname{dim} \operatorname{Aut}(\Sigma, \underline{p}) \\
& =c_{1}(T M) \cdot \beta+\left(\operatorname{dim}_{C} M-3\right)(1-g)+n,
\end{aligned}
$$

where we calculated the first two terms contribution by (7.43) and the last two by (7.42) with addition of moduli for marked points.

This formula reflects the special rôle of Calabi-Yau threefolds. By (5.39) the moduli space of the contributions to the zero point functions $\mathscr{F}^{(g)}(t)$ for all genera is zero dimensional, which reduces the problem of evaluating them to a problem of counting points, albeit a very complicated one. All topological theories will simplify in this way as the example in 2.1. That does not mean in general that all topological observable are integers, because discrete automorphism groups of the theory, which have to be identified in the path integral, weigh some of these points with $1 /|A u t|$ factors. The remarkable fact about $\mathrm{CY}$ threefolds is that an infinite number of physically relevant objects can be reduced in this way. Further comments about the A-model coupling to gravity are exhibited in comparison with the $B$-model in Sec. 6.11.

\subsection{Idea of localisation and the vertex}

The successful setup of this point counting problem in the A-model is a very sophisticated problem, which needs several lectures in its own. Let us mention just a few issues and ideas with references to the literature. In the $A$-model we have a counting problems for each topological type of map $x: \Sigma_{g} \rightarrow M$ which are labeled by $g$ and the class $\beta \in H_{2}(M, \mathbf{Z})$. The virtual dimension of the moduli space might be zero, but points have no hair. They must be characterized by starting with the space of all possible deformations $\mathscr{M}$ and analyze the obstructions. A natural way to describe the obstructions especially if one comes from the path integral would be to give a top dimensional integrand on $\mathscr{M}$ say $c_{\text {top }}$. For instance the question for the topological Euler number is point counting problem asking for the zero set of the generic section $\sigma$ in the tangent bundle, a $\mathscr{C}^{\infty}$ vector field. We can use the Gauss-Bonnet theorem see Sec. 7.3 and write this as $\chi=\int_{\mathscr{M}} c_{n}=\int_{\mathscr{M}} R(g) \mathrm{d} V$. This is not a simplification, unless we have a good choice for $g$ to perform the integral, which comes up if $\mathscr{M}$ admits symmetries. For instance on the sphere we can generate a vector field by rotating the sphere. This introduces a coordinate direction $\phi$ and we can choose the altitude $\theta$ as the second and pick the diagonal constant metric in these coordinates, which is flat everywhere but has $\delta$ curvature at the poles, which leads to the Euler number 2. The poles come of course from the two zeros of the vector field generated by the group action on $S^{2}$, which also leads to the conclusion 
that $\chi=2$. Points that contribute to the integrals can be singled out as fixpoints under group actions $^{21}$. The underlying principle is called localization. The key is to give the points additional structure which describes the group action in its neighborhood in a way that is useful to address global cohomological questions, like calculating intersections. Learning about the group and the target from the action of the group is a highly developed subject[17, 43]. As far as the cohomology is concerned it is systematized in equivariant cohomology. An example of principal importance in the A-model are group actions of the algebraic torus $T=\left(\mathbf{C}^{*}\right)^{r}, \mathbf{C}^{*}=\mathbf{C} \backslash\{0\}$. In equivariant cohomology the cohomology of point is already a very rich structure. E.g. under the torus action it is, see [76] chap 4.2 for an introduction,

$$
H_{T}^{*}(\{p t\})=H^{*}(B T)=H^{*}\left(\left(\mathbf{P}^{\infty}\right)^{r}\right)=\mathbf{Q}\left[t_{1}, \ldots, t_{r}\right]
$$

the polynomial ring over the rationals in $r$ variables thought as $t_{i}=c_{1}\left(H\left(C^{\infty}\right)\right)$, see Sec. 7.3.

Toric varieties of $\operatorname{dim} r$ are varieties on which the algebraic torus $\left(\mathbf{C}^{*}\right)^{r}$ acts on open subsets. They are completely characterized by the degenerations of the $\left(\mathbf{C}^{*}\right)^{r}$ action which are given by an $r$ valent graph $\Gamma$. Locally the degenerations of toric 3 fold can be represented by a 3 vertex, which represents an $\mathbf{C}^{3}$ for the torus action $z_{i} \rightarrow \lambda_{i} z_{i}$, with $\lambda_{i} \in \mathbf{C}^{*}$. Every toric 3 fold can be build by patching $C^{3}$ patches in a way compatible with the global $\left(\mathbf{C}^{*}\right)^{3}$ action. The torus action of the ambient space can be pushed forward to the moduli space of the maps $\mathscr{M}(\beta, g)$ and allows to define an equivariant cohomology theory on $\mathscr{M}(\beta, g)$, by which the points can be enumerated using the Atiyah-Bott fixpoint formula [6]. This technique was pioneered by Kontsevich in [100] for pedagogical introduction and further references see [76]. An interesting point is that the correlations functions of critical bosonic worldsheet gravity appear very explicitly in the formulas for the equivariant virtual fundamental class over which [100] integrates in $\mathscr{M}(\beta, g)$.

The most effective method to solve the open and closed topological string on open toric CalabiYau manifolds employs the connection of open topological string to Chern-Simons theory [148]. This leads to the construction of the topological vertex [1] as reviewed in more detail in [114]. The topological vertex amplitude is the building block for calculation any closed or open string amplitude in any toric CY variety by

- Solving the general problem on a $\mathbf{C}^{3}$ patch for arbitrary conditions on three stacks of $D$ branes on Harvey-Lawson special Lagrangian cycles with topology $S^{1} \times \mathbf{R}^{2}$ as in Fig. 12 This amplitude can be calculated in terms of the large $N$ expansion of link invariants $W_{R R^{\prime}}(q)$ of Chern-Simons theory on $S^{3}$ [1]. In a specific framing one has

$$
Z_{R_{1} R_{2} R_{3}}(q)=\sum_{R, Q_{1}, Q_{2}} N_{Q_{1}, R}^{R_{1}} N_{Q_{3}^{t} R}^{R_{3}^{t}} q^{\kappa_{R_{2}} / 2+\kappa_{R_{3}} / 2} \frac{W_{R_{2}^{t} Q_{1}}(q) W_{R_{2} Q_{3}^{t}}(q)}{W_{R_{2}}(q)},
$$

where $N_{R_{1} R_{2}}^{R_{3}}$ are the usual tensor product coefficients and $\kappa_{R}=\sum_{i} l_{i}\left(l_{i}-2 i+1\right)$ and $l_{i}$ is the length of the row of the $i^{\prime}$ th line in the Young-Tableaux of $R$. Note that $q=e^{\lambda}$ with $\lambda$ the string coupling. i.e. $Z_{R_{1}, R_{2}, R_{3}}$ is exact in $q$ and contains all genus information. All possible boundary conditions on the stack of $N D$-branes are encoded in $R$.

\footnotetext{
${ }^{21}$ Another way to single them out is a critical points of a Morse function.
} 


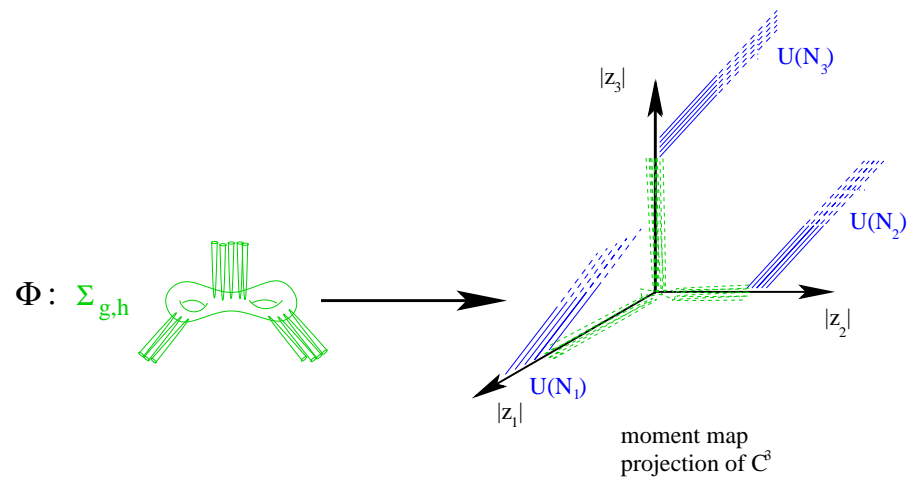

Figure 12: Moment map projection of the vertex and an amplitude with genus 2 and boundary conditions specified by three representations $R_{i}$ of $U\left(N_{i}\right)$ of three stack of D-branes wrapping Harvey-Lawson special Lagrangian cycles of topology $S^{1} \times \mathbf{R}^{2}$.

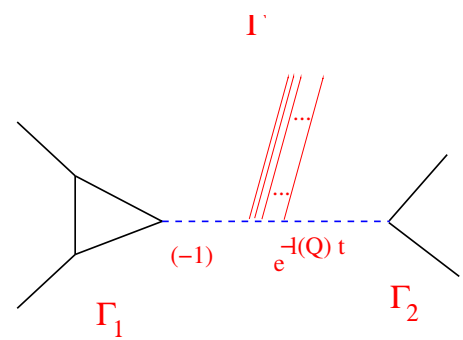

Figure 13: Gluing of graphs along a connecting propagator

- Providing gluing rules: If $\Gamma=\Gamma_{1} \cup \Gamma_{2}$ and $X_{\Gamma_{i}}$ are the associated toric varieties then

$$
Z\left(X_{\Gamma}\right)=\sum_{Q} Z\left(X_{\Gamma_{L}}\right)_{Q}(-1)^{l(Q)} e^{-l(Q) t} Z\left(X_{\Gamma_{L}}\right)_{Q^{t}}
$$

with $t$ is the Kähler parameter "size" of the connecting $\mathbf{P}^{1}$. The quantity $(-1)^{l(Q)} e^{-l(Q) t}$, with $l(Q)$ the number of boxes in the Young-Tableaux of the intermediate representation, can be viewed as propagator. Here again we ignore the data of the framing, which are essential to patch together arbitrary toric varieties.

For instance the Calabi-Yau geometry $\mathscr{O}(-3) \rightarrow \mathbf{P}^{2}$ is covered by three patches, with the moment map projection as in Fig. 14 The partition function $Z_{\mathbf{P}^{2}}$ for closed strings is obtained by gluing three vertices with trivial representation $Q_{i}=\cdot$ on the outer legs by three propagators

$$
Z_{\mathbf{P}^{2}}=\sum_{R_{1}, R_{2}, R_{3}}(-1)^{\sum_{i} l\left(R_{i}\right)} e^{-\sum_{i} l\left(R_{i}\right) t} q^{\sum_{i} \kappa_{R_{i}}} Z_{\cdot R_{2} R_{3}^{t}} Z_{\cdot R_{1} R_{2}^{t}} Z_{\cdot R_{3} R_{1}^{t}} .
$$

All $t$ represent the volume of the hyperplane $\mathbf{P}^{1}$, so that $t$ is the single Kähler parameter of $\mathscr{O}(-3) \rightarrow$ $\mathbf{P}^{2}$.

The calculation is easily performed and by taking the logarithm we get the generating function for the all genus contribution

$$
\mathscr{F}(\lambda, \underline{t})=\sum_{\lambda=0}^{\infty} \lambda^{2 g-2} \mathscr{F}^{(g)}(\underline{t})
$$




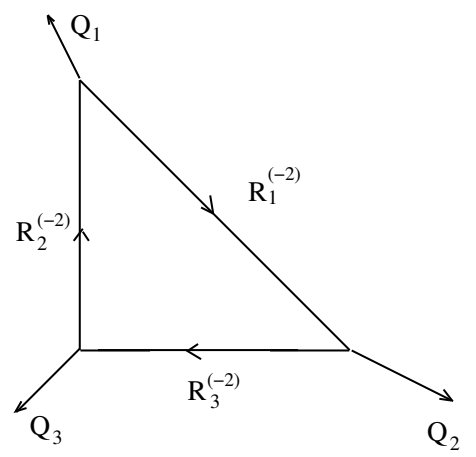

Figure 14: The moment map projection that shows the degeneration of the torus action $\left(\mathbf{C}^{2}\right)^{*}$ on $\mathscr{O}(-3) \rightarrow$ $\mathbf{P}^{2}$

All $\mathscr{F}^{(g)}$ have an expansion $\mathscr{F}^{(g)}=\sum_{\beta} r_{\beta}^{g} q^{\beta}$, where the $r_{\beta}^{g} \in \mathbf{Q}$ are the Gromow-Witten invariants for the holomorphic map from $\Sigma_{g}$ to a curve in the class $\beta \in H_{2}(M, \mathbf{Z})$ of the image curve in $M$.

\subsection{The Gopakumar-Vafa invariants}

The authors of [66] consider an M-theory compactification on $M$ to five dimensions. The space time BPS states fall into representations of the little group of the $5 \mathrm{~d}$ Lorentz group $L=$ $\mathrm{SO}(4) \simeq \mathrm{SU}(2)_{L} \times \mathrm{SU}(2)_{R}$. The low energy interpretation of the free energy $F$ in $4 \mathrm{~d}$ relates it to the $5 \mathrm{~d}$ BPS spectrum through a Schwinger one loop calculation of the $4 \mathrm{~d} R_{+}^{2} F_{+}^{2 g-2}$ effective terms. A similar one loop calculation corrects the effective gauge coupling $\frac{1}{g^{2}\left(G, p^{2}\right)}$ through threshold effects [89]. Note that these $4 \mathrm{~d}$ calculations are sensitive to the off shell quantum numbers, i.e. to $\mathrm{SU}(2)_{L} \times \mathrm{SU}(2)_{R}$. Only BPS particles annihilated by the supercharges in the $\left(\mathbf{0}, \frac{\mathbf{1}}{2}\right)$ representation contribute to the loop. They couple to the anti-selfdual graviphoton field strength $F_{+}$and the antiselfdual curvature $R_{+}$only via their left spin eigenvalues of their representation under $L$. The right representation content enters solely via its multiplicity and a sign $(-1)^{2 j_{R}^{3}}$, in particular any contribution of long multiplets is projected out by these signs. To summarize, the dependence of $\mathscr{F}$ on the BPS spectrum is via a supersymmetric index

$$
I(\alpha, \beta)=\operatorname{Tr}_{\mathscr{H}}(-1)^{2 j_{L}^{3}+2 j_{R}^{3}} e^{-\alpha j_{L}^{3}-\beta H}
$$

and all information entering $\mathscr{F}$ is carried by the following combination

$$
\sum_{j_{L}^{3}, j_{R}^{3}}(-1)^{2 j_{R}^{3}}\left(2 j_{R}^{3}+1\right) N_{j_{R}^{3}, J_{L}^{3}}^{\beta}\left[\mathbf{j}_{\mathbf{L}}\right]=\sum_{g=0}^{\infty} n_{\beta}^{(g)} I_{g} .
$$

of the multiplicities of the BPS states $N_{j_{R}^{3}, J_{L}^{3}}^{\beta}$. The last basis change of the left spin from $\left[\mathbf{j}_{\mathbf{L}}\right]$ to

$$
I_{g}=\left[\left(\frac{\mathbf{1}}{\mathbf{2}}\right)_{L}+2(\mathbf{0})_{L}\right]^{\otimes g}
$$

relates the left spin to the genus $g$ of $C$ and defines the integer Gopakumar-Vafa invariants $n_{\beta}^{(g)}$ associated to a holomorphic curve $C$ of genus $g$ in the class $\beta=[C] \in H^{2}(M, \mathbf{Z})$. In contrast to the $n_{\beta}^{(g)}$, the $N_{j_{R}^{3}, j_{L}^{3}}^{\beta}$ are no symplectic invariants. They change when lines of marginal stability 
in the complex moduli space are crossed ${ }^{22}$ [90]. The $N_{j_{R}^{3}, j_{L}^{3}}^{\beta}$ are interpreted as the dimension of $\mathrm{SU}(2)_{L} \times \mathrm{SU}(2)_{R}$ representations w.r.t. a natural action of this group on the cohomology of the moduli space of the M2 brane. This moduli space is given by the moduli of flat $\mathrm{U}(1)$ connections on $C$ and the moduli of the curve. A model for this space is the Jacobian fibration over the moduli space of the curve $C$ in $\beta$. The expansion of $\mathscr{F}^{\text {hol }}$ in terms of these BPS state sums is obtained by performing the Schwinger loop calculation [\#, [66] as

$$
\begin{aligned}
\mathscr{F}^{\mathrm{hol}}(\lambda, t) & =\sum_{g=0}^{\infty} \lambda^{2 g-2} \mathscr{F}^{(g)}(t) \\
& =\frac{c(t)}{\lambda^{2}}+l(t)+\sum_{g=0}^{\infty} \sum_{\beta \in H_{2}(M, \mathbf{Z})} \sum_{m=1}^{\infty} n_{\beta}^{(g)} \frac{1}{m}\left(2 \sin \frac{m \lambda}{2}\right)^{2 g-2} q^{\beta m} \\
& =\frac{c(t)}{\lambda^{2}}+l(t)+\sum_{g=0}^{\infty} \sum_{\beta \in H_{2}(M, \mathbf{Z})} \sum_{m=1}^{\infty} n_{\beta}^{(g)}(-1)^{g-1} \frac{[m]^{(2 g-2)}}{m} q^{\beta m}
\end{aligned}
$$

with

$$
q^{\beta}=e^{i \sum_{i=1}^{h^{1,1}} t_{i} \int_{\beta} J_{i}}, \quad[x]:=q_{\lambda}^{\frac{x}{2}}-q_{\lambda}^{-\frac{x}{2}}, \quad q_{\lambda}=e^{i \lambda} .
$$

The cubic term $c(t)$ in the Kähler parameters $t_{i}$ is the classical part of the prepotential $\mathscr{F}^{(0)}$ given in 6.84) without the constant term, and $l(t)=\sum_{i=1}^{h} \frac{t_{i}}{24} \int_{M} \operatorname{ch}_{2} J_{i}$ is the classical part of $\mathscr{F}^{(1)}$. Using the expansion

$$
\frac{1}{m} \frac{1}{\left(2 \sin \frac{m \lambda}{2}\right)^{2}}=\sum_{g=0} \lambda^{2 g-2}(-1)^{g+1} \frac{B_{2 g}}{2 g(2 g-2) !} m^{2 g-3}
$$

and a $\zeta(x)=\sum_{m=1}^{\infty} \frac{1}{m^{x}}$ regularization of the sum over $m$ with $\zeta(-n)=-\frac{B_{n+1}}{n+1}$, we see that for $g \geq 2$ the $\beta=0$ constant map terms from localization [51]

$$
\langle 1\rangle_{g, 0}^{M}=(-1)^{g} \frac{\chi}{2} \int_{\mathscr{M}_{g}} c_{g-1}^{3}=(-1)^{g} \frac{\chi}{2} \frac{\left|B_{2 g} B_{2 g-2}\right|}{2 g(2 g-2)(2 g-2) !}
$$

are reproduced if we set $n_{0}^{(0)}=-\frac{\chi}{2}$. This choice also reproduces the constant term proportional to $\zeta(3)$ in $\mathscr{F}^{(0)}$. In $\mathscr{F}^{(1)}$ there is a $\zeta(1)$ term which requires an additional regularization.

In terms of the invariants $n_{\beta}^{(g)}$ the partition function $Z^{\text {hol }}=\exp (\mathscr{F}$ hol $)$ has the following product form $^{23}$

$$
Z_{\mathrm{GV}}^{\mathrm{hol}}\left(M, q_{\lambda}, q\right)=\prod_{\beta}\left[\left(\prod_{r=1}^{\infty}\left(1-q_{\lambda}^{r} q^{\beta}\right)^{r n_{\beta}^{(0)}}\right) \prod_{g=1}^{\infty} \prod_{l=0}^{2 g-2}\left(1-q_{\lambda}^{g-l-1} q^{\beta}\right)^{(-1)^{g+r}\left(\begin{array}{c}
2 g-2 \\
l
\end{array}\right) n_{\beta}^{(g)}}\right] .
$$

This product form resembles the Hilbert scheme of symmetric products written in terms of partition sums over free fermionic and bosonic fields with an integer $U(1)$ charge as well as the closely related product form for the elliptic genus of symmetric products. As it has already been pointed out in [65], it is also reminiscent of the Borcherds product form of automorphic forms of $O(2, n, \mathbf{Z})$, see [15] and [101] for a review. Here the idea is that integrality of the $n_{\beta}^{(g)}$ is related to the fact that they are Fourier coefficients of other (quasi)automorphic forms, see also [91].

\footnotetext{
${ }^{22}$ Notice that the successful microscopic interpretation of the $5 \mathrm{~d}$ black hole entropy requires deformation invariance and relies on the index-like quantity and not on the total number of BPS states.

${ }^{23}$ Here we dropped the $\exp \left(\frac{c(t)}{\lambda^{2}}+l(t)\right)$ factor of the classical terms at genus 0,1 .
} 


\subsection{Relation to Donaldson-Thomas Invariants}

Another way to obtain BPS states is by wrapping D-branes on supersymmetric cycles in $M$. In particular, we can wrap Euclidean 6-branes on $M$ itself and Euclidean 2-branes on a curve $C \subset M$, possibly bound to some 0-branes. At the level of RR charges such a configuration can be cast into a short exact sequence of the form

$$
0 \longrightarrow \mathscr{I} \longrightarrow \mathscr{O}_{M} \longrightarrow \mathscr{O}_{Z} \longrightarrow 0
$$

where $\mathscr{I}$ is the ideal sheaf describing this configuration and $Z$ is the subscheme of $M$ consisting of the curve $C$ and the points at which the 0-branes are supported. Counting BPS states therefore leads to the study of the moduli space $I_{m}(M, \beta)$ of such ideal sheaves $\mathscr{I}$, which has two discrete invariants: the class $\beta=[Z] \in H_{2}(M, \mathbf{Z})$ and, roughly speaking, the number of 0-branes $m=\chi\left(\mathscr{O}_{Z}\right)$. Due to the Calabi-Yau condition the virtual dimension of $I_{m}(M, \beta)$ is zero, and the number of BPS states with these charges is therefore obtained by counting the points in $I_{m}(M, \beta)$. It is, however, not quite as simple as that because as is well-known from Gromov-Witten theory, these configurations can appear in families, and one has to work with the virtual fundamental class. Putting this important subtlety aside, this number is called the Donaldson-Thomas invariant $\tilde{n}_{\beta}^{(m)}$ [47], [138]. These invariants are expected to be integral as they count BPS states.

Since both invariants, Gopakumar-Vafa and Donaldson-Thomas, keep track of the number of BPS states, they should be related. The relation is in fact a consequence of the S-duality in topological strings [119], and takes the following form. The factor in 5.51) coming from the constant maps gives the McMahon function $M\left(q_{\lambda}\right)=\prod_{n \geq 0} \frac{1}{\left(1-q_{\lambda}^{n}\right)^{n}}$ to the power $\frac{\chi}{2}$. This function appears also in Donaldson-Thomas theory [112], calculable on local toric Calabi-Yau spaces e.g. with the vertex [1]. However, in Donaldson-Thomas theory the power of the McMahon function is $\chi$. Note also that if (5.48) holds then $\mathscr{F}$ or $Z$ restricted to this class is always a finite degree rational function in $q_{\lambda}$ symmetric in $q_{\lambda} \rightarrow \frac{1}{q_{\lambda}}$, since the genus is finite in a given class $\beta$. Thanks to this observation one can read from the comparison of the expansion of $Z^{\text {hol }}$ in terms of DonaldsonThomas invariants $\tilde{n}_{\beta}^{(m)} \in \mathbf{Z}$

$$
Z_{\mathrm{DT}}^{\mathrm{hol}}\left(M, q_{\lambda}, q\right)=\sum_{\beta, m \in \mathbf{Z}} \tilde{n}_{\beta}^{(m)} q_{\lambda}^{m} q^{\beta}
$$

with the expansion in terms of Gopakumar-Vafa invariants [112]

$$
Z_{\mathrm{GV}}^{\mathrm{hol}}\left(M, q_{\lambda}, q\right) M\left(q_{\lambda}\right)^{\frac{\chi(M)}{2}}=Z_{\mathrm{DT}}^{\mathrm{hol}}\left(M,-q_{\lambda}, q\right)
$$

the precise relation between $\tilde{n}_{Q}^{(m)}$ and $n_{Q}^{(g)}$. Eq. (5.48) and (5.51) then relate the two types of invariants to the Gromov-Witten invariants $r_{Q}^{(g)} \in \mathbf{Q}$ as in

$$
\mathscr{F}_{\mathrm{GW}}^{\mathrm{hol}}(\lambda, q)=\sum_{g=0}^{\infty} \lambda^{2 g-2} \sum_{\beta} r_{\beta}^{(g)} q^{\beta}
$$

\section{The topological $B$-model}

Since the axial $U(1)_{A}$, whose gauge connection is added to the spin connection to define the $B$-model, develops an anomaly of its current proportional to $\int_{\Sigma} \partial_{\mu} j_{A}^{\mu} \sim \int_{\Sigma} x^{*}\left(c_{1}(T M)\right)$ the twisted 
$B$-model is only consistent for Kähler manifold with vanishing first Chern class, i.e. Calabi-Yau manifolds.

Our plan for the treatment of the B-model is as follows. In next two sections we will present the principal structure of the topological $B$-model and its coupling to gravity. We will then recall some facts about families of complex manifolds. The integrability of the complex structure deformations on Calabi-Yau manifolds will be presented in some detail following the proof of Tian, partly because it is one of the main classical results, but also because it leads directly to the formulation of Kodaira-Spencer theory of gravity. The behavior of the periods under infinitesimal deformations of the complex structure is the preparation for the derivation of the special Kähler geometry relation from geometry. After that we discuss two methods to obtain the Picard-Fuchs equations, which play a central role to actually solve the B-model. The quintic hypersurfaces is the main example, however we aim for a presentation, which paves the way for generalizations to the bulk of the known Calabi-Yau: complete intersections in weighted projective space.

\subsection{The topological $B$ without worldsheet gravity}

The scalar BRST operator is in this case, see table 3 .

$$
Q_{B}=\bar{Q}_{-}+\bar{Q}_{+} .
$$

The scalar fields on the worldsheet are conveniently chosen as

$$
\eta^{\bar{\imath}}:=-\left(\psi_{-}^{\bar{\imath}}+\psi_{+}^{\bar{\imath}}\right), \quad \theta_{j}:=g_{j \bar{\imath}}\left(\psi_{+}^{\bar{\imath}}-\psi_{-}^{\bar{\imath}}\right),
$$

while the one form fields are

$$
\rho_{z}^{i}:=\psi_{-}^{i} \quad \text { of type }(1,0), \quad \rho_{\bar{z}}^{i}:=\psi_{+}^{i} \quad \text { of type }(0,1) .
$$

The supersymmetry transformation $\delta=\bar{\varepsilon} \bar{Q}_{+}+\bar{\varepsilon} \bar{Q}_{-}$is obtained by setting $\bar{\varepsilon}_{+}=-\bar{\varepsilon} \bar{\varepsilon}_{-}=\bar{\varepsilon}$ and $\varepsilon_{ \pm}=0$

$$
\begin{aligned}
\delta x_{i} & =0, & \delta x^{\bar{l}} & =\bar{\varepsilon} \eta^{\bar{\imath}} \\
\delta \theta_{i} & =0, & \delta \eta^{\bar{l}} & =0 \\
\delta \rho_{\mu}^{i} & = \pm i \bar{\varepsilon} \partial_{\mu} x^{i} . & &
\end{aligned}
$$

The zero form observables $\mathscr{O}^{(0)}$ are now related to forms in $\Omega^{(0, p)}\left(M, \Lambda^{q} T^{1,0} M\right)$ with the identification of the scalar Grassmann fields on the worldsheet to forms and vectors on $M \eta^{\bar{l}} \leftrightarrow \mathrm{d} x^{\bar{l}}$ and $\theta_{i} \leftrightarrow \frac{\partial}{\partial x^{i}}$. I.e. to each form on $M$ of type

$$
W=\omega_{\bar{l}_{1} \ldots \bar{l}_{p}}^{j_{1} \ldots j_{q}} \mathrm{~d} x^{\bar{\tau}_{1}} \wedge \ldots \wedge \mathrm{d} x^{\bar{t}_{p}} \frac{\partial}{\partial x^{j_{1}}} \wedge \ldots \wedge \frac{\partial}{\partial x^{j_{q}}}
$$

we associate a 0 -form operator on $\Sigma$

$$
\mathscr{O}_{W}^{(0)}=\omega_{\bar{l}_{1} \ldots \bar{l}_{p}}^{j_{1} \ldots j_{q}} \eta^{\bar{l}_{1}} \ldots \eta^{\bar{l}_{p}} \theta_{j_{1}} \ldots \theta_{j_{q}} .
$$

One checks that the $Q_{B}$ operator is identified with the Dolbeault operator $\bar{\partial}$ which increases the anti holomorphic form degree

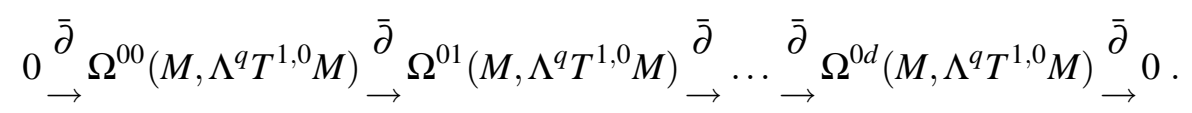


and one has with $\left\{Q_{B}, \mathscr{O}_{W}^{(1)}\right\}=-\mathscr{O}_{\bar{\partial} W}^{(0)}$ the identification

$$
H_{Q_{B}}^{*}=\frac{\operatorname{Ker} Q_{B}}{\operatorname{Im} Q_{B}}=\bigoplus_{p, q=0}^{d} H^{0, p}\left(M, \Lambda^{q} T^{1,0} M\right) .
$$

The selection rules from the $R$-symmetries are as before $\sum_{i} p_{i}=\sum_{i} q_{i}=d(1-g)$. It follows that for $g=0$ we have again the possibility of a non-vanishing three point function $\left\langle\mathscr{O}_{A^{(i)}} \mathscr{O}_{A^{(j)}} \mathscr{O}_{A^{(k)}}\right\rangle$, if we consider three local operators $\mathscr{O}_{A^{(k)}}$ associated to

$$
A^{(k)}=\omega_{\bar{j}}^{(k) i} \mathrm{~d} x^{\bar{j}} \frac{\partial}{\partial x^{i}} \in H^{1}\left(M, T^{1,0} M\right) .
$$

Eq. (6.4) shows that there is a fixpoint of the fermionic symmetry at the constant maps

$$
\partial_{\mu} x^{i}=0 \text {. }
$$

We expect therefore that all contributions to the path integral are localized to constant maps. This is the main simplification in the $B$-model. For constant maps $\Sigma_{g}$ is mapped to a point in $M$. These maps are of course much easier to control then the holomophic maps of the $A$-model and in particular they are not affected by the sizes, i.e. Kähler parameter of $M$. The $B$-model without worldsheet gravity is like a Kaluza-Klein reduction. By writing the action in the form

$$
S=i t \int_{\sigma}\left\{Q_{B}, V\right\}+t W
$$

with

$$
V=g_{i \bar{j}}\left(\rho_{z} \partial_{\bar{z}} x^{\bar{j}}+\rho_{\bar{z}}^{i} \partial_{z} x^{\bar{j}}\right)
$$

and

$$
W=\int_{\Sigma_{g}}\left(-\theta_{i} D \rho^{i}-\frac{i}{2} R_{i \bar{i} j} \rho^{i} \wedge \rho^{j} \eta^{\bar{i}} \theta_{k} g^{\bar{j} k}\right)
$$

one can conclude the following. $W$ does not depend on the complex structure of $\Sigma$, which decouples from the B-model at genus 0 . The Kähler variations of $W$ are $Q_{B}$ exact and decouple likewise. It is also $t$ independent as $t$ can be absorbed in a field redefinition in $W$. For more details see [156]. In the off shell formulation of [105]106] one can simply write the complete action as $Q$ commutator $S=\left\{Q_{B}, \tilde{V}\right\}$ which makes the above points more obvious.

Since the fixpoints of the fermionic maps of the $B$-model are constant maps, mapping all $\Sigma$ to a point in the Calabi-Yau manifold $M$, their moduli space contains $M$ and in the special case of the three punctured sphere, i.e. in the case of the three point function it is actually $M$, since these three points can be fixed on $S^{2}$ by an $S L(2, \mathbf{C})$ transformation and the sphere itself has no complex deformations. For this reason all we have to find is a canonical measure on $M$, which we integrate over $M$ to get the three point function. Using Kaluza Klein reduction methods this measure has been found long ago [133]

$$
C_{i j k}(z)=\left\langle\mathscr{O}_{A^{i}}^{(0)} \mathscr{O}_{A^{j}}^{(0)} \mathscr{O}_{A^{k}}^{(0)}\right\rangle=\int_{M} \Omega \wedge A_{\bar{j}_{1}}^{(i) i_{1}} A_{\bar{j}_{2}}^{(j) i_{2}} A_{\bar{j}_{3}}^{(k) i_{3}} \Omega_{i_{1} i_{2} i_{3}} \mathrm{~d} x^{\bar{j}_{1}} \wedge \mathrm{d} x^{\bar{j}_{2}} \wedge \mathrm{d} x^{\bar{j}_{3}}
$$

Here $\Omega(z)$ is the unique non-vanishing holomorphic $(3,0)$ form, which exists on every CalabiYau, see Sec. (7.5). Using the isomomorphism (6.22) $A \mapsto \hat{A}$ we can define a non-holomorphic two point function

$$
N_{i \bar{j}}=\int_{M} \hat{A}^{(i)} \wedge \overline{\hat{A}}^{(\bar{j})}
$$




\subsection{First order complex structure deformation}

The expressions (6.14) and (6.15) depend as anticipated only on the complex structure of $M$ and not on its Kähler structure. We saw in section 4.3 that deformations of the action by $\int_{\Sigma} \mathscr{O}_{A^{k}}^{(2)}$ with $A^{(k)} \in H_{\bar{\partial}}^{(0,1)}(M, T M)$ are first order complex structure deformations of $M$. Our aim is to explain in this section the local tangent space of the complex structure moduli space from a different point of view, put forward by Kodaira and Spencer [98] and to explain in the next section why the first order deformations on a Calabi-Yau manifold are unobstructed.

Consider a $2 n$ real dimensional manifold and a covering of it by coordinate patches $\mathscr{U}_{i}, i=$ $1, \ldots, r$, which are homeomorphic to a neighborhood $U_{i} \in \mathbf{C}^{n}$ with coordinates $x_{\alpha}^{(i)}(p), \alpha=1, \ldots, n$. It is a complex manifold if the transition functions $f^{(j k)}: x^{(k)}(p) \rightarrow x^{(j)}(p)$, defined for $p \in \mathscr{U}_{j} \cap \mathscr{U}_{k}$, are biholomorphic. One attempts to define a family of complex manifolds $M_{z}$, by considering a family of transition functions $x_{\alpha}^{(j)}=f_{\alpha}^{(j k)}\left(x^{(k)}, z\right)$, which depend also holomorphically on the complex parameters $z$. The difficulty is that some $z$ dependence of $f_{\alpha}^{(i k)}\left(x^{(k)}, z\right)$ corresponds just to different choices of local coordinates systems on the same complex manifold. In order to decide whether the $f^{(j k)}\left(x^{(k)}, z\right)$ really induce changes of the complex structure [98] considers in every patch $U_{k}$ an infinitesimal coordinate change that is characterized by a holomorphic vector field $V^{(k)}(z)=\sum_{\alpha=1}^{n} \frac{\partial f_{\alpha}^{(k)}\left(x^{(k)}, z\right)}{\partial z} \frac{\partial}{\partial x_{\alpha}^{(k)}}$. Next consider the composition of transition functions in $\mathscr{U}_{i} \cap \mathscr{U}_{j} \cap$ $\mathscr{U}_{k}$. By definition

$$
f_{\alpha}^{(i k)}\left(x^{(k)}, z\right)=f_{\alpha}^{(i j)}\left(f_{1}^{(j k)}\left(x^{(k)}, z\right), \ldots, f_{n}^{(j k)}\left(x^{(k)}, z\right), z\right)
$$

holds. Differentiation w.r.t. to $z$ gives

$$
\frac{\partial f_{\alpha}^{(i k)}\left(x^{(k)}, z\right)}{\partial z}=\frac{\partial f_{\alpha}^{(i j)}\left(x^{(j)}, z\right)}{\partial z}+\sum_{\beta=1}^{n} \frac{\partial x_{\alpha}^{(i)}}{\partial x_{\beta}^{(j)}} \frac{\partial f_{\beta}^{(j k)}\left(x^{(k)}, z\right)}{\partial z}
$$

Denote general vector fields by

$$
A^{(j k)}(z)=\sum_{\alpha=1}^{n} \frac{\partial f_{\alpha}^{(j k)}\left(x^{(k)}, z\right)}{\partial z} \frac{\partial}{\partial x_{\alpha}^{(j)}}, \quad x^{(k)}=f^{(k j)}\left(x_{j}, z\right)
$$

Note that $A^{(k k)}(z)=0$ since $f_{\alpha}^{(k k)}=x^{(k)}$ independently of $z$. Therefore eq. (6.17) written covariantly in terms of the vector fields (6.18) implies $A^{(k j)}(z)=-A^{(j k)}(z)$. For general $i, j, k$ (6.17) is a Čhech 1-cocycle condition for the $A^{(i j)}$

$$
A^{(i j)}(z)+A^{(k i)}(z)+A^{(j k)}(z)=0
$$

The exact 1-cocycles come precisely from the infinitesimal coordinates changes setting $A^{(j k)}(z)=$ $V^{(j)}(z)-V^{(k)}(z)$, while the true changes of complex structure correspond to 1-cocycles, which are not exact, i.e. elements of $H^{1}(M, A)$, where $A$ are sheaves of vector fields $A=\mathscr{O}(T M)$. The ČheckDolbeault theorem $(7.10)$ with $F=\mathscr{O}(T M)$ implies that complex structure deformations are given by elements in $H^{0,1}(M, T M)$, which we also call $A$. 


\subsection{Unobstructedness of the complex deformation space}

As explained in [98] the existence of a global complex structure deformation requires the vanishing of higher Čhech cohomology groups for vector fields. Tian [139] and Todorov [142] have proven that these higher order conditions are automatically fulfilled on a Calabi-Yau space.

The elements $A(z)=A_{\bar{j}}^{i}(x, z) \mathrm{d} x^{\bar{j}} \frac{\partial}{\partial x^{i}}$ in $H^{(0,1)}(M, T M)$ in the complex moduli space can be used to deform the $\bar{\partial}$ operator to $\bar{\partial}_{z}=(\bar{\partial}+A(z))$ so that $\bar{\partial}_{z} f(x)=0$, defines what a holomorphic function on $M$ is w.r.t. the new complex structure. The requirement that $\bar{\partial}_{z}^{2}=0$ leads to

$$
\bar{\partial} A(z)+\frac{1}{2}[A(z), A(z)]=0,
$$

where [., .] is the Lie bracket. For $\phi(x)=\phi^{i}(x) \partial_{x_{i}} \in \mathscr{L}^{0, p}(T)$, with $\phi^{i}=\frac{1}{p !} \phi(x)_{\bar{l}_{1}, \ldots, \bar{l}_{p}}^{i} \mathrm{~d} x^{\bar{\tau}_{1}} \wedge \ldots \wedge \mathrm{d} x^{\bar{\tau}_{p}}$, and $\omega(x) \in \mathscr{L}^{0, q}(T)$ similarly defined one has

$$
[\phi, \omega]=\left(\phi^{i} \wedge \partial_{i} \omega^{j}-(-1)^{p q} \omega^{i} \wedge \partial_{i} \phi^{j}\right) \partial_{j},
$$

giving above a $(0,2)$ form vector field from two $(0,1)$-form vector fields. Condition (6.20) is equivalent to the vanishing of the Nijenhuis tensor (7.5) [98].

The main idea of the proof is that the existence of the holomorphic $(n, 0)$ form induces an isomorphism

$$
H^{(0, p)}(M, T M) \cong H^{n-1, p}(M) .
$$

under which the condition (6.20) is converted into a cohomological question, which is solved by the $\partial \bar{\partial}$ lemma. This conversion of the deformation problem to a cohomological question, which is solved by an analog of the $\partial \bar{\partial}$ Lemma extends to deformations of $G_{2}$ metrics [87][84].

Contraction with the homolomorphic $(n, 0)$ form associates to $A=A_{\bar{j}_{1}, \ldots, \bar{j}_{p}}^{i} \mathrm{~d} x^{\bar{j}_{1}} \wedge \ldots \wedge \mathrm{d} x^{\bar{j}_{p}} \frac{\partial}{\partial x^{i}} \in$ $H^{(0, p)}(M, T M)$ an $\hat{A} \in H^{n-1, p}(M)$ as

$$
\hat{A}=\frac{1}{(n-1) !} A_{\bar{j}_{1}, \ldots, \bar{j}_{p}}^{j} \Omega_{j, i_{2}, \ldots, i_{n}} \mathrm{~d} x^{i_{2}} \wedge \ldots \wedge \mathrm{d} x^{i_{n}} \mathrm{~d} x^{\bar{j}_{1}} \wedge \ldots \wedge \mathrm{d} x^{\bar{j}_{p}}
$$

with the inverse

$$
(\hat{A})^{\vee}=\frac{1}{(n-1) !|\Omega|^{2}} \bar{\Omega}^{i, i_{2}, \ldots, i_{n}} \hat{A}_{i_{2}, \ldots i_{n}, \bar{j}_{1}, \ldots, \bar{j}_{p}} \mathrm{~d} x^{\bar{j}_{1}} \wedge \ldots \wedge \mathrm{d} x^{\bar{j}_{p}} \frac{\partial}{\partial x^{i}}
$$

where $|\Omega|^{2}$ is defined in $(7.52$. One checks that $A$ is harmonic iff $\hat{A}$ is harmonic and the operation is invertible i.e. $A=\left(A^{\wedge}\right)^{\vee}$, which shows (6.22).

Since $\Omega$ is holomorphic the hat operation (6.23) commutes with $\bar{\partial}$ and we get

$$
\bar{\partial} \hat{A}=\widehat{\bar{\partial} A}=-\frac{1}{2} \widehat{[A, A]}=:-\frac{1}{2}[\hat{A}, \hat{A}],
$$

as equivalent to the condition (6.20).

The main technical instrument is the following Lemma

$$
[\hat{A}, \hat{B}]:=\widehat{[A, B]}=\partial \widehat{(A \wedge B})-(D \cdot A) \wedge \hat{B}+\hat{A} \wedge(D \cdot B),
$$

where $D \cdot A=\left(\partial_{i} A_{\bar{j}_{1} \ldots \bar{j}_{p}}^{i}\right) x^{\bar{j}_{1}} \wedge \ldots \wedge \mathrm{d} x^{\bar{j}_{p}}$ is a contraction. The calculation is a straightforward exercise whose solution is made explicit in [139]. Eq. (6.26) becomes particularly useful, if one can choose 
"gauge" representatives for $A$ and $B$ so that $(D \cdot A)=(D \cdot B)=0$. To control this "gauge" condition Tian considers a Taylor expansion $A(z)=A_{1} z+A_{2} z^{2}+\ldots$ with $A_{i}$ sections of $\Gamma\left(M, \Omega^{(0,1)}(T M)\right)$ and starting data $\bar{\partial}_{0}=\bar{\partial}$, i.e. $A(0)=0$. To order $z$ (6.20) states $\bar{\partial} A_{1}(x)=0$ and we already argued that in order to get rid of complex coordinate transformations we should consider $A_{1} \in H_{\bar{\partial}}^{(0,1)}(M, T M)$ only. One wants now to prove inductively that $\partial A_{k}+\frac{1}{2} \sum_{i=1}^{k-1}\left[A_{i}, A_{k-i}\right]=0$ for $k>1$ which by (6.25) is equivalent to

$$
\bar{\partial} \hat{A}_{k}=\frac{1}{2} \sum_{i=1}^{k-1}\left[\hat{A}_{i}, \hat{A}_{k-i}\right], \quad \text { for } k>1
$$

First step of induction: To first order in $z$ one has simply as above $\hat{A}_{1} \in H^{n-1,1}(M)$ and we pick the harmonic representative $\hat{A}_{1}$. In fact on compact Kähler manifolds it follows from (7.20,7.24) that every harmonic representative fulfills $\bar{\partial} A_{1}=\bar{\partial}^{*} A_{1}=0$. Moreover with $\Delta_{\bar{\partial}}=\Delta_{\partial}$, see sect. 7.2 also $\partial \hat{A}_{1}=0$ holds. This implies $D \cdot A_{1}=0$ and by $(6.26)\left[\hat{A}_{1}, \hat{A}_{1}\right]=\partial\left(\widehat{A_{1} \wedge A_{1}}\right)$ is $\partial$-exact. On the other hand for $\hat{A}_{1} \in H^{n-1,1}(M)$ hence $\bar{\partial} A_{1}=0$ it is immediate from the definition of the bracket that $\bar{\partial}\left[\hat{A}_{1}, \hat{A}_{1}\right]=\bar{\partial} \partial\left(\widehat{A_{1} \wedge A_{1}}\right)=0$. The $\partial, \bar{\partial}$ Lemma of Kähler geometry ([72], p 149) states that if a form $\eta \in \Omega^{p, q}$ is $\bar{\partial}$ closed and d-, $\partial$ - or $\bar{\partial}$ - exact then it can be written as $\eta=\partial \bar{\partial} \psi$. Applied to the bracket we can write $\left[\hat{A}_{1}, \hat{A}_{1}\right]=\partial \bar{\partial} \psi_{1}$ for some $\psi_{1} \in \Omega^{1,1}$. Identifying $\hat{A}_{2}=\frac{1}{2} \partial \psi_{1}$ we have constructed a solution to $\bar{\partial} \hat{A}_{2}+\frac{1}{2}\left[\hat{A}_{1}, \hat{A}_{1}\right]=0$.

General induction: If for some $N$ one has solved for $\hat{A}_{i}$ with $\partial \hat{A}_{i}=0$ and $\bar{\partial} \hat{A}_{i}+\frac{1}{2} \sum_{j=1}^{i-1}\left[\hat{A}_{j}, \hat{A}_{i-j}\right]=$ $0, i=1, \ldots, N$, then

$$
\sum_{j=1}^{N}\left[\hat{A}_{j}, \hat{A}_{N+1-j}\right]=\partial \sum_{j=1}^{N}\left(A_{j} \wedge A_{N+1-j}\right)^{\wedge}
$$

and one also checks that

$$
\begin{aligned}
\bar{\partial}\left(\sum_{j=1}^{N}\left[\hat{A}_{j}, \hat{A}_{N+1-j}\right]\right) & =\bar{\partial} \partial\left(\sum_{j=1}^{N}\left[A_{j}, A_{N+1-j}\right]\right)^{\wedge} \\
& =\frac{1}{2} \partial\left(\sum_{j=1}^{N} \sum_{k=1}^{j-1}\left[\left[A_{k}, A_{j-k}\right], A_{N+1-j}\right]-\left[A_{j},\left[A_{k}, A_{N+1-j-k}\right]\right]\right)^{\wedge}=0 .
\end{aligned}
$$

Here we used first (6.26), then the fact that $\bar{\partial}$ and $\wedge$ commutes, (6.28) for $A_{k}$ with $k \leq N$ and the Jacobi identity for (6.21). By the $\partial, \bar{\partial}$ Lemma one can set $\hat{A}_{N+1}=\frac{1}{2} \partial \psi_{N}$ and since $\partial \hat{A}_{N+1}=0$ the induction proceeds. Moreover one has arguments that the series converges in $H^{n-1,1}(M)$ [139].

Hence there exists always a family of Calabi-Yau manifolds with varying complex structure parameters, whose complex dimension is $h^{(0,1)}(M, T M)$. Tians and Todorov's result is very important also with respect to the world sheet theory, where is very not-trivial to establish that a deformation of type (4.18) is exactly marginal and does lead to family of $N=2$ SCFTs.

Mirror statement. On a Calabi-Yau threefold one has the above mentioned isomorphism between $H^{(0,1)}(M, T M)$ and $H^{2,1}(M)$, which is induced by the unique $(3,0)$ form $\Omega$. Thanks to the above isomorphism the $A$-model and $B$-model physical operators are associated to $H^{p, q}$ and the mirror symmetry can be interpreted as the following identification of these spaces $H^{p, q}(M) \leftrightarrow$ $H^{d-p, q}(W)$. Here $M$ and $W$ are mirror manifolds. As a corollary one has $\chi(M)=-\chi(W)$ if $d$ is odd. 

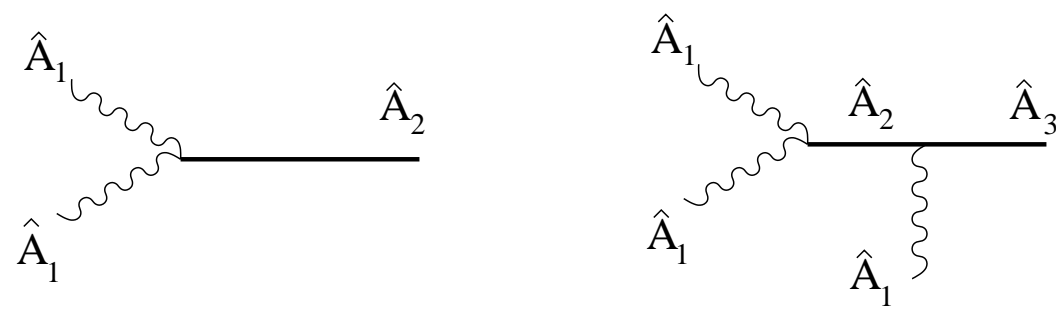

Figure 15: Perturbative solution of the Kodaira-Spence equation in Tians form $\bar{\partial} A(z)+\frac{1}{2} \partial(A(\widehat{z) \wedge A}(z))=0$ by Feynmann graphs with massless fields (weavy lines) and massive fields (solid lines).

\subsection{Kodaira-Spencer gravity as space-time action for the B-model}

There are three space time actions known, which reproduce as classical equations of motion the unobstructedness of complex structures on the Calabi-Yau. Kodaira-Spencer gravity [11], Hitchins three-form action [84] and Hitchins general threeform action [85]. The first[11] and the last [125] reproduce the B-model also at one loop. But even Einstein's gravity poses no problem up to one loop [141]. While it is not clear how the suggested spacetime descriptions make sense as full quantum theory, the worldsheet B-model approach makes remarkable predictions at higher loops.

Kodaira-Spencer theory of gravity is a theory on $M$ which couples exclusively to the complex moduli of $M$. Its tree level result reproduces the $B$-model without the coupling to worldsheet gravity, i.e. its genus zero sector[11]. It is a space time gravity theory in the sense that is does couple to the Calabi-Yau metric as far as complex structure dependence is concerned. It reproduces the (6.20) in the form $\bar{\partial} A(z)+\frac{1}{2} \partial(A(\widehat{z) \wedge A}(z))=0$ as its equation of motion and its Feynman graph expansion corresponds to the iterative solution to that equation exactly in the form as given above. In fact by the $\partial, \bar{\partial}$-Lemma we have shown e.g. in the second induction step that one has an $\psi_{1}$ with $\partial \bar{\partial} \psi_{1}=\left[\widehat{A_{1}, A_{1}}\right]$, hence $\hat{A}_{2}=\frac{1}{2} \partial \psi_{1}$. By $(\widehat{6.26})$ the first statement means also $\bar{\partial} \psi=\left(\widehat{A_{1} \wedge A_{1}}\right)$. Combining the two facts one gets a solution for $\hat{A}_{2}$ in the form

$$
\hat{A}_{2}=-\frac{1}{2 \bar{\partial}} \partial\left(\widehat{A_{1} \wedge A_{1}}\right)=\mathscr{P}\left(\widehat{A_{1} \wedge A_{1}}\right)
$$

We have used a "gauge" $\partial \hat{A}_{k}=0$ and it is easy to see that the recursive solution comes with the freedom $\hat{A}_{k}+\bar{\partial} \lambda$, which one can fix be requiring $\bar{\partial}^{*} A_{k}=0$. We can then define the "propagator" as $\mathscr{P}=-\frac{1}{2 \bar{\partial}} \partial=-\bar{\partial}^{*} \frac{1}{2 \Delta_{\bar{\partial}}} \partial$. With this "propagator" one can recursively write the solutions to $\hat{A}_{k}$. E.g. $\hat{A}_{3}=2 \mathscr{P}\left(A_{1} \wedge\left(\mathscr{P}\left(\widehat{A_{1} \wedge A_{1}}\right)\right)^{\vee}\right)^{\wedge}$. It follows from the construction of $A_{k}$ that only $\hat{A}_{1}$ fulfills the Laplace equation, while $A_{k}$ for $k>1$ correspond to "massive modes."

It is not hard to see [11], that the Kodaira-Spencer action

$$
\lambda^{2} S\left(\hat{A}_{1}, \hat{A}_{m}, z_{0}\right)=\int_{M} \frac{1}{2} \hat{A}_{m} \mathscr{P} \hat{A}_{m}+\frac{1}{6}\left(\left(A_{1}+A_{m}\right) \wedge\left(A_{1}+A_{m}\right)\right)^{\wedge} \wedge\left(A_{1}+A_{m}\right)^{\wedge}
$$

has $\bar{\partial}\left(\hat{A}_{1}+\hat{A}_{m}\right)+\frac{1}{2} \partial\left(\left(A_{1}+A_{m}\right) \wedge\left(A_{1}+A_{m}\right)\right)^{\wedge}=0$ as e.o.m. and reproduces the Feynman graph expansion above. Here we have defined as $A_{m}$ the massive part of $A(z)$ and $z_{0}$ is the background value of the complex structure. It has further been shown that (5.30) is the reduction of closed string field theory to the topological modes and it has been argued that its path integral defines the generating function for all correlators of the topological B-model coupled to worldsheet gravity. 
However the action does not make sense as quantum theory. So its solution is indirect by means of the holomorphic anomaly equation of the topological B-model. Nevertheless the divergent factors in the graph expansion of (6.30) lead to an analysis of the leading behavior at the boundaries of the complex moduli space of the Calabi-Yau space once the ones of the three point couplings are known. For one modulus $t$ one gets $F_{g} \sim \frac{\left[\partial_{t}^{3} C_{t t t}{ }^{2 g-2}\right.}{\left[\partial_{t} C_{t t t}\right]}$. This result is useful to fix the holomorphic ambiguity.

\subsection{The periods and infinitesimal deformations of complex structure}

The integral (6.14) can expressed in terms of holomorphic functions on the complex moduli space parametrized by $z$, which are integrals of the holomorphic $(3,0)$-fom over a fixed topological basis of three cycles of $M$

$$
X^{k}(z)=\int_{A_{k}} \Omega(z), \quad F_{k}(z)=\int_{B^{k}} \Omega(z), \quad k=0, \ldots, h_{2,1} .
$$

These are called period integrals or periods for short. Here we have chosen an integral symplectic basis of $A$ and $B$ cycles of the integral homology $H_{3}(M, \mathbf{Z})$ such that $A_{k} \cap B^{l}=\delta_{k}^{l}$, while $A^{i} \cap A^{j}=$ $B_{i} \cap B_{j}=0$. The choice of such a basis in $H_{3}(M, \mathbf{Z})$ and its dual basis $\left(\alpha_{i}, \beta^{j}\right)$ in the integral cohomology $H^{3}(M, \mathbf{Z})$ with

$$
\int_{M} \alpha_{k} \wedge \beta^{l}=\int_{A_{l}} \alpha_{k}=-\int_{M} \beta^{l} \wedge \alpha_{k}=-\int_{B_{k}} \beta^{l}=\delta_{k}^{l}
$$

is unique up to an $\operatorname{Sp}\left(h^{3}, \mathbf{Z}\right)$ transformation. The two dual symplectic bases $\left(A^{k}, B_{k}\right)$ and $\left(\alpha_{i}, \beta^{j}\right)$ are topological and do in particular not depend on the complex structure. What we call $(n, 0)$ form $\Omega(z)$ does depend on the complex structure. This dependence is captured by the period integrals, w.r.t to the fixed basis $\left(\alpha_{i}, \beta^{j}\right)$

$$
\Omega(z)=X^{k}(z) \alpha_{k}-F_{k}(z) \beta^{k} .
$$

The symplectic group over $\mathbf{C}$ is defined by

$$
M^{\dagger} \Sigma M=\Sigma, \quad M \in S p\left(h^{3}, \mathbf{C}\right) \quad \text { with } \quad \Sigma=\left(\begin{array}{cc}
0 & \mathbf{1} \\
-1 & 0
\end{array}\right) .
$$

$\Omega$ is a symplectic invariance and we have a natural action on the period vector

$$
\Pi:=\left(\begin{array}{c}
X^{k} \\
F_{k}
\end{array}\right) \quad \text { by } \quad \tilde{\Pi}=M \Pi .
$$

The $X^{k}$ are homogeneous projective coordinates of the complex structure moduli space and one can choose locally inhomogeneous coordinates

$$
t^{k}=\frac{X^{k}}{X^{0}} \quad k=1, \ldots, h:=h^{2,1}
$$

as the complex structure parameters[71] 139]. This can be viewed as local Torelli theorem for Calabi-Yau manifolds. A global Torelli is proven for $K 3$ (and Enriques surfaces) [7], but seems not to hold on general Calabi-Yau manifolds. 
In virtue of (6.36) the $F_{k}$ must be expressible as functions of $t$. The precise relation comes from the infinitesimal calculus describing changes of the $(n, 0)$-form $\Omega$ in $H^{n}(M)$ under changes of the complex structure. The decomposition of $H^{n}(M)$ into $(p, q)$ type $H^{n}(M)=\oplus_{p+q=n} H^{p, q}(M)$ varies over the complex moduli space parametrized by $t$. We are concerned with $n=3$. One wants to describe the varying of $H^{p, q}\left(M_{t}\right)$ as a bundle $\mathscr{H}^{p, q}$ over the moduli domain $D(M)$ of $M$, called the Hodge bundle. However the spaces $H^{p, q}$ do not fiber holomorphically over $D(M)$. One defines therefore first a Hodge filtration $\mathbf{F}^{*}(M)=\left\{\mathbf{F}^{p}(M)\right\}_{p=0}^{n}$ by $\mathbf{F}^{p}(M)=\bigoplus_{a \geq p} H^{a, k-a}(M)$, with $H^{n}(M, \mathbf{C})=\mathbf{F}^{p}(M) \oplus \overline{\mathbf{F}^{k-p+1}(M)}$. Obviously $H^{p, q}(M)$ is recovered as $H^{p, q}(M)=\mathbf{F}^{p}(M) \cap \overline{\mathbf{F}^{q}(M)}$ and one has an isomorphism $H^{p, q}(M)=\mathbf{F}^{p}(M) / \mathbf{F}^{p+1}(M)$. The $\mathbf{F}^{p}\left(M_{t}\right)$ form holomorphic bundles $\mathscr{F}^{p}$ over $D(M)$ and the holomorphic Hodge bundle $\mathscr{H}^{p, q}$ can be defined as $\mathscr{H}^{p, q}=\mathscr{F}^{p} / \mathscr{F}^{p+1}$, see [73] for a precise definition of $D(M)$. There is a bilinear form on $H^{n}(M, \mathbf{Z}) /$ torsion

$$
Q(\phi, \psi)=(-1)^{n(n-1) / 2} \int_{M} \phi \wedge \psi
$$

with the following properties

$$
\begin{array}{r}
Q\left(H^{p q}, H^{p^{\prime} q^{\prime}}\right)=0, \quad \text { unless } p^{\prime}=n-p \text { and } q^{\prime}=n-q \\
S(\psi, \psi) \equiv i^{p-q} Q(\psi, \bar{\psi}) \quad>0, \quad \text { unless } \psi=0 \text { in } H^{p, q} .
\end{array}
$$

In mathematical terms $Q$ is called a polarization on the Hodge structure $H^{n}(M, \mathbf{Z}) /$ torsion and (6.38) and 6.39) are the first and second Riemann bilinear relations, see [72, 73]. In particular $\mathscr{H}^{3,0}$ defines a line subbundle $\mathscr{L}$ in $H^{3}(M)$ and $\Omega(z)$ defines a section of it. Since it is expandable in the fixed integer frame $\left(\alpha_{k}, \beta^{l}\right)$ by the periods (6.33) it has a flat connection that is called GaussManin connection. The Picard-Fuchs equations that the periods fulfill, which we derive later, can be viewed as one manifestation of the flatness of the Gauss-Manin connection. Despite the fact that the connection is flat the period vector $\Pi(6.35)$ will have a monodromy $G \in \operatorname{Sp}\left(h^{3}, \mathbf{Z}\right)$, if transported around loops $\Gamma_{z_{0}}$ encircling singular points $z_{i}$ in the complex moduli space. To understand the possibility of a monodromy remember that the moduli space is not simply connected. Singular or orbifold loci of $M$ are cut out. As exemplified at the end of Sec. 7.4 not simply connected manifolds can have non trivial holonomy of flat connections ${ }^{24}$. The monodromy group is generated by transport around all loops $\gamma_{i}$ in $H^{1}(\mathscr{M})$

$$
\Pi(z)=M_{\gamma_{i}} \Pi(z), \quad M_{\gamma_{i}} \in S p\left(h^{3}, \mathbf{Z}\right),
$$

where one has relations, e.g. in the situation depicted in figure 16 one has $M_{\gamma_{\infty}}^{-1}=M_{\gamma_{0}} M_{\gamma_{1}}$. The homotopy group of $\mathscr{M}$ and the symplectic monodromies around the loops determine the period vector as solution to a Riemann-Hilbert problem.

By taking a derivative w.r.t. the complex structure coordinates $z^{k}$ the $(3,0)$ form changes as follows

$$
\frac{\partial \Omega}{\partial z^{k}}=c_{k}(z) \Omega+\hat{A}^{(k)},
$$

where $c_{k}(z)$ depends only on the complex moduli and $\hat{A}^{(k)} \in H^{2,1}$. This can be seen as follows. Let, as in section (6.2), $f^{\mu}(x, z)$ define a family of holomorphic coordinates on $M$, which vary

\footnotetext{
${ }^{24}$ This holonomy is called a "Wilson line" in physics.
} 

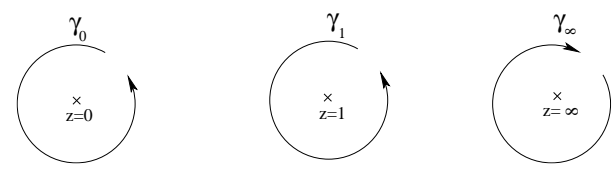

Figure 16: Moduli space of a one complex parameter Calabi-Yau manifold compactified to $\mathbf{P}^{1}$ with three singular points. In general singularities are divisors in $\mathscr{M}$.

with the complex structure parameter $z$, so that $x^{\mu}=f^{\mu}\left(x, z_{0}\right)$. Via $f^{\mu}(x, z)$ the $(3,0)$-form $\Omega=$ $\frac{1}{3 !} h(f) \varepsilon_{\mu v \rho} \mathrm{d} f^{\mu} \mathrm{d} f^{v} \mathrm{~d} f^{\rho}$ depends on the complex structure $z$ and by derivation we get

$$
\frac{\partial \Omega}{\partial z^{k}}=\frac{1}{3 !} \frac{\partial h}{\partial z^{k}} \varepsilon_{\mu v \rho} \mathrm{d} f^{\mu} \mathrm{d} f^{v} \mathrm{~d} f^{\rho}+\frac{1}{2 !} h \varepsilon_{\mu v \rho} \mathrm{d} f^{\mu} \mathrm{d} f^{v} \frac{\partial\left(\mathrm{d} f^{\rho}\right)}{\partial z^{k}} .
$$

To analyze $\frac{\partial\left(\mathrm{d} f^{\rho}\right)}{\partial z^{k}}$ requires an infinitesimal calculus in the neighborhood of the reference complex structure $z_{0}$. It is easy to convince oneself that the $\left.(0,1) \operatorname{part} \frac{\partial\left(\mathrm{d} f^{\rho}\right)}{\partial z^{k}}\right|_{(0,1)}=A_{\bar{j}}^{(k) \rho} \mathrm{d} z^{\bar{j}}$, where $A^{(k)} \in$ $H^{(0,1)}\left(M, T^{1,0} M\right)$ is the object we encountered in Sec. 6.2. The isomorphism (6.22) implies then (6.41). Upon taking further derivatives we get

$$
\begin{aligned}
\frac{\partial}{\partial X^{i}} \Omega \in \mathbf{F}^{2} & =H^{3,0} \oplus H^{2,1} \\
\frac{\partial^{2}}{\partial X^{i} \partial X^{j}} \Omega \in \mathbf{F}^{1} & =H^{3,0} \oplus H^{2,1} \oplus H^{1,2} \\
\frac{\partial^{3}}{\partial X^{i} \partial X^{j} \partial X^{k}} \Omega \in \mathbf{F}^{0} & =H^{3,0} \oplus H^{2,1} \oplus H^{1,2} \oplus H^{0,3} .
\end{aligned}
$$

\subsection{Special Kähler geometry}

Let us discuss the consequences of the first property ( 6.38 ), which follows from simple consideration of type. If we insert (6.33) in $\int_{M} \Omega \wedge \frac{\partial}{\partial X^{k}} \Omega=0$, a consequence of (6.43) and 6.38), we can conclude that $F_{k}=\frac{1}{2} \frac{\partial}{\partial X_{k}} \sum_{i} X^{i} F_{i}$. That implies that the $F_{i}$ are indeed not independent but determined as derivatives of the single function ${ }^{25}$

$$
F=\frac{1}{2} \sum_{i=0}^{h} X^{i} F_{i}
$$

called the prepotential. Note that $F$ is not a symplectic invariant. It follows further from the first transversality that $F$ is homogeneous of degree 2 in $X^{a}$, i.e. $\sum_{a=0}^{h} X^{a} \frac{\partial}{\partial X_{a}} F=2 F$. The implication of the second line in (6.43) $\int_{M} \Omega \wedge \frac{\partial^{2}}{\partial X_{i} \partial X_{j}} \Omega=0$ follows already from the degree two homogeneity of $F$ and contains no new information. The last line of (5.43) shows that $\int_{M} \Omega \wedge \frac{\partial^{3}}{\partial_{\bar{X}} a \overline{\bar{X}}^{b} \partial_{\bar{X}}} \Omega$ is nonzero and we calculate

$$
C_{a b c}(t)=\int_{M} \Omega \wedge \frac{\partial^{3}}{\partial_{\hat{X}^{a}} \partial_{\hat{X}^{b}} \partial_{\hat{X}^{c}}} \Omega=\frac{\partial^{3}}{\partial_{\hat{X}^{a}} \partial_{\hat{X}^{b}} \partial_{\hat{X}^{c}}} F=\left(X^{0}\right)^{2} \frac{\partial^{3}}{\partial_{\hat{t}_{a}} \partial_{\hat{t}_{b}} \partial_{\hat{t}_{c}}} \mathscr{F}^{(0)}(t),
$$

\footnotetext{
${ }^{25}$ Note that on even complex dimensional Calabi-Yau manifolds there will be no relative sign in 6.32 basis nor in 6.33) and $\int_{M} \Omega \wedge \Omega=2 X^{a} F_{a}=0$ gives already an algebraic relation between the periods. Using further transversalities one find an intriguing mix between algebraic and differential relations between the periods in the even case.
} 
where $a, b, c$ runs form 1 to $h^{21}$. To derive this we used 6.31, 6.32, 5.33 and the homogeneity of degree two of $F$ to pass to the inhomogeneous variables $t$. Each of the three derivatives w.r.t. to the complex structure parameters $\frac{\partial}{\partial X^{k}}$ has to hit one $\mathrm{d} f^{\kappa}$ in $\Omega=\frac{1}{3 !} h(f) \varepsilon_{\mu v \rho} \mathrm{d} f^{\mu} \mathrm{d} f^{v} \mathrm{~d} f^{\rho}$ to produce the $(0,3)$ part. It is clear by (6.42) that the eq. (6.45) is up to a normalization equivalent to (6.14). It turns out that mirror symmetry identifies $C_{a b c}(t)=\frac{\partial^{3}}{\partial_{t_{a}} \partial_{t_{b}} \partial_{t_{c}}} \mathscr{F}(0)(t)$ at a special point in the moduli space with (5.12). The right hand side of 6.45) is not covariant. It is valid only in the coordinate system defined by the periods $X^{a}$ or the inhomogeneous coordinates $t^{a}$. The period expression is however valid in any parametrization of the complex structure. If we make a coordinate transformations of the latter $X^{a} \rightarrow z^{a}(X)$ we need no covariant derivatives on the right hand side to compensate for the derivatives of $\frac{\partial z^{a}}{\partial X^{b}}$ by $z$, because by (6.43) only terms contribute, for which all derivatives by $z$ act on $\Omega(z)$. In any complex structure coordinates we can therefore express the triple couplings in terms of the period integrals as

$$
C_{i j k}=\int \Omega \wedge \partial_{i} \partial_{j} \partial_{k} \Omega=\sum_{l=0}^{h}\left(X^{l} \partial_{i} \partial_{j} \partial_{k} F_{l}-F_{l} \partial_{i} \partial_{j} \partial_{k} X^{l}\right)
$$

and $C_{i j k}$ transforms like $\mathrm{Sym}^{3} T \mathscr{M} \otimes \mathscr{L}^{-2}$ under Kähler- and general coordinate transformations in the complex moduli space $\mathscr{M}$. Note that $C_{i j k}$ is by (6.35) a symplectic invariant, if the derivative is w.r.t. to invariant complex structure parameters, such as the $z$ in Sec. 6.7. The triple coupling are the Yukawa couplings of the moduli fields in the effective action of heterotic string compactifications, see e.g. [67, 126].

Let us come to the two point function (6.15) and is relation to (6.39). As we have discussed the $(3,0)$ form $\Omega(z)$ lies a complex line bundle $\mathscr{H}^{3,0}$. This bundle is called the vacuum bundle $\mathscr{L}$ in physics. Is has a natural gauge transformation $\Omega \mapsto e^{f(z)} \Omega$ where $f(z)$ is holomorphic, which leads to another nowhere vanishing $(3,0)$ form. We have by $(5.39)$ a positive hermitian norm $S(\Omega, \Omega)=\|\Omega\|^{2}:=i \int_{M} \Omega \wedge \bar{\Omega}$, which is is related to the norm (7.52) by a volume factor $\|\Omega\|^{2}=i V|\Omega|^{2}$. We define a now potential

$$
K=-\log i \int_{M} \Omega \wedge \bar{\Omega}
$$

which will turn out to be Kähler potential of the moduli space metric. Clearly the gauge transformation become Kähler transformations $K \mapsto K-f-\bar{f}$ and $e^{K}$ is a section of real line bundle. We can define a candidate Kähler metric on the moduli space

$$
G_{a \bar{b}}=\partial_{a} \bar{\partial}_{\bar{b}} K
$$

Note by (7.33) that the Kähler form to this metric is the curvature form $\mathscr{R}$ of the hermitian metric $S(\Omega, \Omega)$ on $\mathscr{L}$. Using (6.41) we can relate this metric to (6.15)

$$
G_{a \bar{b}}=-\frac{\int_{M} \hat{A}^{(a)} \wedge \hat{A}^{(\bar{b})}}{\int_{M} \Omega \wedge \bar{\Omega}} .
$$

These couplings (6.15) are the kinetic terms of the moduli fields [67, 126].

Let us compare that metric $G_{a \bar{b}}$ with the standard way one defines a metric on the space of metrics on $M$. The metric on the Calabi-Yau moduli space factorizes at least locally in the Kähler- 
and the complex structure deformations space, see Sec. 4.3 and [23, 22] for further background,

$$
2 G_{a \bar{b}} \delta z^{a} \boldsymbol{\delta} z^{\bar{b}}=\frac{1}{2 V} \int_{M} g^{m \bar{n}} g^{k \bar{l}} \delta g_{m k} \delta g_{\bar{n} \bar{l}} \operatorname{det}\left(g_{a b}\right)^{\frac{1}{2}} \mathrm{~d} x^{6},
$$

where we just took the complex structure deformations into account. The metric (6.50) is called the Weil-Peterson metric of the complex moduli space. In Sec. 4.3 we have already identified pure deformations of the metric with elements in $H^{1}(M, T M)$, the precise relation is $\delta g_{\bar{m} \bar{n}}^{(a)}=\frac{\partial g_{\bar{n} \bar{m}}}{\partial z^{a}} \delta z^{a}=$ $-2 A_{\bar{n}}^{(a)}{ }^{i} g_{i \bar{n}} \delta z^{a}$. Using (6.24) in (6.50) we note the remarkable fact that the two metrics (5.48) and (6.50) coincide. This was first proven in [139] and implies the local Torelli theorem as well as the fact that the holomorphic sectional curvature of the Weil-Peterson metric is negative and bounded away form zero [139].

From (6.47) and (6.33) follows a simple formula for its Kähler potential in terms of the periods

$$
K=-\log i\left(\bar{X}^{\bar{a}} \frac{\partial F}{\partial X^{a}}-X^{a} \frac{\partial \bar{F}}{\partial \bar{X}^{\bar{a}}}\right) .
$$

This statement in terms of the inhomogeneous coordinates $t_{i}=X^{i} / X^{0}, i=1, \ldots, h^{2,1}$ reads

$$
e^{-K(t, \bar{t})}=\left(t^{i}-\bar{t}^{\bar{\imath}}\right)\left(\partial_{i} \mathscr{F}^{(0)}-\bar{\partial}_{\bar{l}}^{\overline{\mathscr{F}}^{(0)}}\right)-2\left(\mathscr{F}^{(0)}-\overline{\mathscr{F}}^{(0)}\right) .
$$

As it obvious the $C_{i j k}(t) \in \operatorname{Sym}^{3} T^{*} \mathscr{M} \otimes \mathscr{L}^{2}$ as well as the real Kähler potential $K(t \bar{t})$ derive from the holomorphic section $\mathscr{F}^{(0)}(t) \in \mathscr{L}^{2}$ over the complex moduli space $\mathscr{M}$. This justifies the name prepotential for $\mathscr{F}^{(0)}$ and the structure defined by (6.48), 6.52) and (6.45) supplemented with the requirement that the Chern class represented by the curvature two form $\mathscr{R}$ of the vacuum line bundle $\mathscr{L}$ defines an even integral class ${ }^{26}$ on $\mathscr{M}$ is known as special Kähler geometry.

The integrability condition for the existence of $\mathscr{F}^{(0)}$, given $G_{i \bar{j}}=\partial_{i} \bar{\partial}_{\bar{j}} K(t, \bar{t})$ and $C_{i j k}$, is

$$
R_{i \bar{k} j}^{l}=-\bar{\partial}_{\bar{k}} \Gamma_{i j}^{l}=\left[D_{i}, \partial_{\bar{k}}\right]_{j}^{l}=G_{i \bar{k}} \delta_{j}^{l}+G_{j \bar{k}} \delta_{i}^{l}-C_{i j m} \bar{C}_{\bar{k}}^{m l}
$$

The upshot of special Kähler geometry is that the relevant quantities are fixed by the section $\mathscr{F}$ of the holomorphic line bundle $\mathscr{L}^{2}$ over the compactified moduli space. As it is well known in complex geometry such sections are fixed by a finite set of data, basically a Riemann-Hilbert problem to find sections of the Hodge-bundle, which observe certain monodromies. This fact underlies our ability to solve the two derivative effective action of $N=2$ gauge theories exactly.

This structure we have discussed here mainly from the geometrical point of view has been independently discovered in the vector multiplet moduli space of $N=2$ supergravity theories in four dimensions [37, 38, 35]. The connection to string compactifications has been made in [22, 135]. In making contact with the supergravity literature note that [37, 38, 35] uses for the homogeneous sections

$$
L^{I}=e^{\frac{K}{2}} X^{I}, \quad M_{I}=\kappa e^{\frac{K}{2}} F_{I},
$$

over $\mathscr{M}$, which are not holomorphic $\partial_{\bar{k}} X^{I}=\bar{\partial}_{\bar{k}} F_{I}=0$, but covariantly holomorphic with respect to the Kähler connection $D_{\bar{k}}=\left(\partial_{\bar{k}}-\frac{1}{2} K_{\bar{k}}\right)$, i.e. $D_{\bar{k}} L^{I}=D_{\bar{k}} M_{I}=0$, with the effect that $i\left(\bar{L}^{I} M_{I} \kappa^{-1}-\right.$

\footnotetext{
${ }^{26} \mathrm{~A}$ Kähler manifold (6.52) whose Kähler form is the curvature two-form $\mathscr{R}$ of line bundle $\mathscr{L}$ representing a class in $H^{2}(\mathscr{M}, \mathbf{Z})$ is called Kähler-Hodge in the mathematical literature. As is was pointed out in [34] the fermions already in $N=1$ susy require that $[\mathscr{R}]$ is an even integral class.
} 
$\left.L^{I} \bar{M}_{I} \bar{\kappa}^{-1}\right)=1$. In particular the earlier literature on $N=2$ black holes [53, 136] uses $\kappa=2 i$, because the gravitino variations have been worked out in this conventions [38]. In the inhomogeneous coordinates $t^{I}=\frac{L^{I}}{L^{0}}=\frac{X^{I}}{X^{0}}$ the Kähler factor cancels.

\subsection{Picard-Fuchs equation from the symmetries of the ambient space}

Let us now discuss an explicit simple example of such a mirror symmetry computation. The principle example is the quintic in the projective space $\mathbf{P}^{4}$, which is discussed in great detail in the paper [25]. It is defined as the zero locus of a homogeneous polynomial of degree 5 in $x_{i}$, e.g.

$$
P=\sum_{i=1}^{5} a_{i} x_{i}^{5}+a_{0} \prod_{i=1}^{5} x_{i}=\sum_{i=1}^{5} x_{i}^{5}-z^{-\frac{1}{5}} \prod_{i=1}^{5} x_{i}=0
$$

The $z$ appears here as one of the 101 possible complex structure deformations of the full family of quintics. A deformation is generated by perturbing $P_{0}=\sum_{i=1}^{5} x_{i}^{5}$ with a parameter multiplying a monomial of degree 5. We count (5) $x_{i}^{5}$, (20) $x_{i}^{4} x_{j}$, (20) $x_{i}^{3} x_{j}^{2}$, (30) $x_{i}^{2} x_{j}^{2} x_{k}$, (30) $x_{i} x_{j} x_{k}^{3}$, (20) $x_{i} x_{j} x_{k} x_{l}^{2}$, (1) $\prod_{i=1}^{5}$, with $i, j, k, l=1, \ldots 5$ hence 126 monomials. Not all of those lead to independent complex structure deformations, because the complex linear transformations of the coordinates $x_{i}$ of $\mathbf{P}^{4}$ leads to completely equivalent forms of the constraint. The group of those has dimension $5^{2}-1$. Finally there is one relation by $P=0$ leading to 101 . The symmetric deformation in (5.55) is chosen with hindsight, because we can see it as the unique complex structure deformation on the mirror manifold of the quintic $W$. The mirror is constructed as $\mathbf{Z}_{5}^{3}$ orbifold of the original quintic $M$. The orbifold is generated by phase rotations on the homogeneous coordinates $\mathbf{P}^{4}$

$$
x_{i} \rightarrow \exp \left(2 \pi i g_{i}^{(\alpha)} / 5\right) x_{i}, \quad \alpha=1,2,3, \quad i=1, \ldots, 5,
$$

with $g^{(1)}=(1,4,0,0,0), g^{(2)}=(1,0,4,0,0)$ and $g^{(3)}=(1,0,0,4,0)$. It leaves precisely the perturbing monomial $\prod_{i=1}^{5} x_{i}$ invariant. This one deformation parameter $z$ can be identified with the one Kähler deformation $t$ of the original quintic $M$ which has Hodge numbers $h^{1,1}=1$ and $h^{2,1}=101$. The one element in $H^{1,1}(M)$ comes from the restriction of the unique Kähler form of $\mathbf{P}^{5}$ to the hyper surface. The 101 elements of $H^{1}(M, T M)$ we counted above and explained their relation to $H^{2,1}(M)$ before.

The holomorphic $(3,0)$ form can be written explicitly in every patch $U_{l}$ of $\mathbf{P}^{4}$ as a residue expression [70]

$$
\Omega(z)=\int_{\gamma} \frac{a_{0} \mu}{P}
$$

where the contour surrounds the single pole at $P=0$ inside $\mathbf{P}^{4}$ and the measure is

$$
\mu=\sum_{k=1}^{5}(-1)^{k} w_{i} x^{i} \mathrm{~d} x^{1} \wedge \ldots \wedge \widehat{\mathrm{d} x^{k}} \wedge \ldots \wedge \mathrm{d} x^{5}
$$

In each coordinate patch $U_{l}, x^{l}=1$ and $\mathrm{d} x^{l}=0$ so the sum (6.58) collapses to a single term. The $w_{i}$ makes (6.58) applicable to hypersurfaces in weighted projective space $W C P\left[w_{1}, \ldots, w_{5}\right]$, which are generalizations of $\mathbf{P}^{4}$, for which $w_{i}=1, i=1, \ldots, 5$. An important consistency condition for $\Omega$ is its invariance under the $\mathbf{C}^{*}$ action $x_{i} \rightarrow \lambda x_{i}$. Let us consider the parametrization of the complex structure by the parameters $a_{i}, i=0, \ldots, 5$ in $P=\sum_{i=1}^{5} a_{i} x_{i}^{5}+a_{0} \prod_{i=1}^{5} x_{i}$. These are redundant 
parameters and can be "gauged" by the $G_{\mathbf{P}^{4}}=P G L(N, \mathbf{C}) \times \mathbf{C}^{*}$ transformation on the homogeneous parameters $\left(x_{1}: \ldots: x_{5}\right)$ of $\mathbf{P}^{4}$ to one parameter. Let us summarize the "gauge invariances" of $\Omega(\underline{a})$ , which are obvious from (6.57) and 6.58).

- It is invariant under the change $a_{i} \rightarrow \rho a_{i}$ with $\rho \in \mathbf{C}^{*}$. Defining the logarithmic derivative $\theta_{i}=a_{i} \frac{\partial}{\partial a_{i}}$, this homogeneity of degree 0 is expressed as

$$
\sum_{i=0}^{5} \theta_{i} \Omega(\underline{a})=0
$$

- It is invariant under the $\mathbf{C}^{*}$ actions $\left(a_{i}, a_{j}\right) \rightarrow\left(\rho^{-5} a_{i}, \rho^{5} a_{j}\right), i, j=1, \ldots, 5$ with $\rho \in \mathbf{C}^{*}$. These are compensated on $P$ by $G_{\mathbf{P}^{4}}$ transformations $\left(x_{i}, x_{j}\right) \rightarrow\left(\rho x_{i}, \rho^{-1} x_{j}\right)$, which leave the form $\mu$ invariant. As differential relations one has

$$
\left(\theta_{i}-\theta_{5}\right) \Omega(\underline{a})=0, \quad i=1, \ldots, 5 .
$$

These two equations mean that $\Omega(\underline{a})=\Omega(z)$ does depend only on the combination $z=-\frac{a_{1} a_{2} a_{3} a_{4} a_{5}}{a_{0}^{5}}$, where we chose the sign for latter convenience. Instead of fixing the gauge immediately we first notice the obvious differential relations

$$
\left(\frac{\partial}{\partial a_{0}}\right)^{5} \frac{\Omega(\underline{a})}{a_{0}}=\left(\prod_{i=1}^{n} \frac{\partial}{\partial a_{i}}\right) \frac{\Omega(\underline{a})}{a_{0}} .
$$

With $\theta_{i}=a_{i} \frac{\partial}{\partial a_{i}}, \theta=z \frac{\mathrm{d}}{\mathrm{d} z}$, the commutator $\left[\theta_{i}, a_{i}^{x}\right]=x a_{i}^{x}$ and $\theta_{0}=-5 \theta$ as well as $\theta_{i}=\theta$ for $i=$ $1, \ldots, 5$ we rewrite

$$
\begin{aligned}
\left(\frac{\theta_{0}}{a_{0}}\right)^{5} \frac{\Omega(\underline{a})}{a_{0}} & =\frac{1}{a_{1} a_{2} a_{3} a_{4} a_{5}}\left(\prod_{i=1}^{5} \theta_{i}\right) \frac{\Omega(\underline{a})}{a_{0}} \\
\frac{a_{1} a_{2} a_{3} a_{4} a_{5}}{a_{0}^{5}}\left(\prod_{k=1}\left(\theta_{0}-k\right)\right) \Omega(\underline{a}) & =\left(\prod_{i=1}^{5} \theta_{i}\right) \Omega(\underline{a}) \\
z \prod_{k=1}(5 \theta+k) \Omega(z) & =\theta^{5} \Omega(z)
\end{aligned}
$$

The last line means that the factorizing differential operator $\mathscr{D}=\theta \mathscr{L}=\theta\left[\theta^{4}-z \prod_{i=1}^{4}(\theta+i)\right]$ annihilates $\Omega(z)$ and it also annihilates the periods

$$
\Pi_{i}(z)=\int_{\Gamma_{i}} \Omega(z)
$$

with $\Gamma_{i} \in H^{3}(W)$. One checks that $\mathscr{L} \Omega(z)$ is already exact, i.e. $\int_{\Gamma_{i}} \mathscr{L} \Omega(z)=0$ so that the periods $\Pi_{i}(z)=\int_{\Gamma_{i}} \Omega(z)$, which correspond to the four independent cycles $\Gamma_{i} \in H_{3}(W)$ are determined by the four solutions of differential equation

$$
\left[\theta^{4}-5 z \prod_{i=1}^{4}(\theta+i)\right] \Pi(z)=0 .
$$

Note that the mirror has $h^{2,1}=1$ and hence 4 elements in the middle cohomology $H^{3}(M, \mathbf{Z})=$ $H^{3,0} \oplus H^{21} \oplus H^{12} \oplus H^{03}$. The four period integrals over the dual four homology 3-cycles, which are 
invariant under the $\mathbf{Z}_{5}^{3}$ group correspond to four independent solutions of eq (5.64). The 3-cycles are in a fixed topological basis of $H^{3}(M, \mathbf{Z})$. This basis is independent of the complex structure. The trick in the derivation of the differential equation was to fix the gauge symmetry at the very end (last line of (6.62)). This result is a considerable simplification in the derivation of the period equations compared with the Griffith reduction method discussed below. The method is adjusted to derive the systems of Picard-Fuchs operators of multi parameter Calabi-Yau hypersurfaces and complete intersections in toric ambient spaces, which have the corresponding $\mathbf{C}^{*}$ actions, see [80][97]. It will give in general as above differential operators allowing for too many solutions, which need to be reduced to lower order differential operators. In the simplest case this is accomplished by factorization. As one example of this type consider the hypersurface of degree 12 in $\mathbf{P}(1,1,2,2,6)$, which has $h^{1,1}(M)=2$ and $h^{2,1}(M)=128$. We $\bmod M$ out by an $\mathbf{Z}_{12} \times \mathbf{Z}_{6} \times \mathbf{Z}_{6}$ acting as

$$
x_{i} \rightarrow \exp \left(2 \pi i g_{i}^{(\alpha)} / 12\right) x_{i}, \quad \alpha=1,2,3, \quad i=1, \ldots, 5,
$$

with $g^{(1)}=(1,11,0,0,0), g^{(2)}=(2,0,10,0,0)$ and $g^{(3)}=(2,0,0,10,0)$. The invariant constraint, which we interpret as mirror admits two complex structure deformations $h^{2,1}(W)=2$

$$
P=a_{1} x_{1}^{12}+a_{2} x_{2}^{12}+a_{3} x_{3}^{6}+a_{4} x_{4}^{6}+a_{5} x_{5}^{2}+a_{0} \prod_{i=1}^{5} x_{i}+a_{6}\left(x_{1} x_{2}\right)^{6}
$$

It is convenient to express the multiplicative relation between the monomials in 6.66 in vectors ${ }^{27}$

$$
l^{(1)}=(-6 ; 0,0,1,1,3,1) \quad l^{(2)}=(0 ; 1,1,0,0,0,-2)
$$

such that equations corresponding to (6.61) are now written as

$$
\prod_{l_{i}^{(b)}<0}\left(\frac{\partial}{\partial a_{i}}\right)^{-l_{i}^{(b)}} \frac{\Omega(\underline{a})}{a_{0}}=\prod_{l_{i}^{(b)}<0}\left(\frac{\partial}{\partial a_{i}}\right)^{l_{i}^{(b)}} \frac{\Omega(\underline{a})}{a_{0}} \quad b=1,2 .
$$

Similar symmetry considerations as above lead to the conclusion that $\Pi(\underline{z})$ depends only on

$$
z_{b}=(-1)^{l_{0}^{(b)}} \prod_{i} a_{i}^{l_{i}^{(b)}}, \quad b=1,2
$$

and the reduction of (6.68) leads after factorization to the differential operators $\theta_{i}=z_{i} \frac{\mathrm{d}}{\mathrm{d} z_{i}}$

$$
\begin{aligned}
& \mathscr{D}_{1}=\theta_{1}^{2}\left(\theta_{1}^{2}-2 \theta_{2}\right)-\prod_{i=0}^{2}\left(6 \theta_{1}-(2 i+1)\right) z_{1} \\
& \mathscr{D}_{2}=\theta_{2}^{2}-\prod_{i=1}^{2}\left(2 \theta_{2}-\theta_{1}-i\right) z_{2} .
\end{aligned}
$$

We will discuss the solution to (6.64,6.70) below.

Let us first perform the integral over the small circle $\gamma$ say in the patch $U_{k}$, i.e. $x_{k}=1$ to bring the expression of the $(n, 0)$ form to one which is familiar from the study of Riemann surfaces. In order to do reduce one integration over $\mathrm{d} x_{i}$ to the residue integration $\int \frac{\mathrm{d} p}{p}=2 \pi i$ we perform a coordinate transformation from $\left(x_{1} \ldots \widehat{x^{k}} \ldots x_{5}\right)$ to $\left(x_{1} \ldots \widehat{x_{k}} \ldots \widehat{x}_{i} \ldots x_{5}, P\right)$ under which the measure

\footnotetext{
${ }^{27}$ They will identified with the generators of the Mori cone in Sec. 6.9.
} 
$\mathrm{d} x_{1} \wedge \ldots \widehat{\mathrm{d} x_{k}} \ldots \wedge \mathrm{d} x_{4}$ goes to $\left(\frac{\partial P}{\partial x_{i}}\right)^{-1} \mathrm{~d} x_{1} \wedge \ldots \widehat{\mathrm{d} x_{k}} \ldots \widehat{\mathrm{d} x_{i}} \ldots \wedge \mathrm{d} x_{5} \wedge \mathrm{d} P$. Because of transversality $\mathrm{d} P=0$ has no common solution with $P=0$ and we can always pick an $k$ and $i$ so that $\left(\frac{\partial P}{\partial x_{i}}\right) \neq 0$ for $P=0$. Therefore the integrand will have a single pole at $\frac{1}{P}$ and integration leads to

$$
\Omega(z)=\frac{a_{0} w_{k} x^{k} \mathrm{~d} x_{1} \wedge \ldots \widehat{\mathrm{d} x_{k}} \ldots \widehat{\mathrm{d} x_{i}} \ldots \wedge \mathrm{d} x_{5}}{\frac{\partial P}{\partial x_{i}}} .
$$

This form of the $(n, 0)$ form is analogous to the well known $(1,0)$ form $\Omega \sim \frac{\mathrm{d} x}{y}$ in the case of an elliptic curve realized as cubic in $\mathbf{P}^{2}$ with the inhomogeneous equation in the $z=1$ patch given in the Weierstrass form $y^{2}=4 x^{3}-g_{2} x-g_{3}$. It can be verified that it is nowhere vanishing [70].

\subsection{Picard-Fuchs equation from the Dwork-Griffith reduction method}

From the formal definition of the period $\Pi(z)=\int_{\Gamma_{i}} \Omega(z)$, with $\Omega$ given in 6.57) we can alternatively derive a fourth order differential equation for the period in terms of the moduli $z$ by the Dwork-Griffiths reduction method. The key observation for this algorithm comes as follows. Consider on the ambient space $\mathbf{P}^{m-1}\left(w_{1}, \ldots, w_{m}\right)$ the $(m-2)$-form

$$
\Phi=\frac{a_{0}}{p^{r}} \sum_{i<j}(-1)^{i+j}\left(w_{j} x_{j} A_{i}-w_{i} x_{i} A_{j}\right) \mathrm{d} x_{1} \wedge \ldots \wedge \widehat{\mathrm{d} x_{i}} \wedge \ldots \wedge \widehat{\mathrm{d} x_{j}} \wedge \ldots \wedge \mathrm{d} x_{n} .
$$

Here $A_{i}(x)$ are homogeneous of degree $d_{i}$ in $x$, i.e. $\sum_{k=1}^{m} x_{k} w_{k} \frac{\partial}{\partial_{k}} A_{i}=d_{i} A_{i}$. We further assume that $c_{1}(M)=0 \leftrightarrow \sum_{i=1}^{m} w_{i}=d$, where $d$ is the homogeneous degree of $P, \sum_{k=1}^{m} x_{k} w_{k} \frac{\partial}{\partial_{k}} P=P d$. With this assumptions the total derivative of $\Phi$ simplifies

$$
\begin{aligned}
\mathrm{d} \Phi= & \sum_{k=1}^{m}\left(\frac{a_{0} r}{P^{r+1}} A_{k} \partial_{k} P-\frac{a_{0}}{P^{r}} \partial_{k} A_{k}\right) \mu \\
& +\frac{a_{0}}{P^{r}} \sum_{j=1}^{m}\left(d(1-r)-w_{i}+d_{i}\right) A_{i}(-1)^{j} \mathrm{~d} x_{1} \wedge \ldots \wedge \widehat{\mathrm{d} x_{j}} \wedge \ldots \wedge \mathrm{d} x_{n} .
\end{aligned}
$$

If we choose now the $A_{j}$ so that $A_{j}=0$ for $j \neq k$ and $d_{k}=d(r-1)+w_{k}$ for $f(x):=A_{k}(x)$ the second term vanishes. In other words if $\frac{\partial}{\partial x_{k}}\left(\frac{f(x) a_{0}}{p^{r}} \mu\right)$ is homogeneous of degree 0 w.r.t. the coordinate weights $w_{i}$ then

$$
\frac{a_{0} r f \partial_{k} P}{P^{r+1}} \mu=\frac{a_{0} \partial_{k} f}{P^{r}} \mu
$$

holds under the integration sign.

Let us mention in passing that for Calabi-Yau manifolds defined by a transversal complete intersections of $s$ polynomials, i.e. as the zero set $P_{1}=\ldots=P_{s}=0$ in a weighted projective space the analog of (6.57) is

$$
\Omega=\int_{\gamma_{a}} \ldots \int_{\gamma_{s}} \prod_{k=1}^{s} \frac{a_{0}^{(k)}}{P_{s}} \mu,
$$

where $\gamma_{i}$ are circles around the $P_{i}=0$ and similar as before $\frac{\partial}{\partial x_{k}}\left(f(x) \prod_{k=1}^{s} \frac{a_{0}^{(k)}}{P_{s}} \mu\right)$ is exact iff it is of total degree zero. This leads to the partial integration rule[0]

$$
\sum_{k \neq j} \frac{n_{k}}{n_{j}-1} \frac{P_{j}}{P_{k}} \frac{f \partial_{i} P_{k}}{\prod_{l=1}^{s} P_{l}^{n_{l}}} \mu=\frac{1}{n_{j}-1} \frac{P_{j} \partial_{i}}{\prod_{l=1}^{s} P_{l}^{n_{l}}} \mu-\frac{f \partial_{i} P_{j}}{\prod_{l=1}^{s} P_{l}^{n_{l}}} \mu
$$


where we omitted the factor $\prod_{k=1}^{s} a_{0}^{(k)}$, which is however of relevance for a scaling argument as in (6.62).

The idea is to take up to four derivatives of the period $\Pi(z)$ w.r.t. the complex structure moduli $z$, and rewrite the emerging expression by the repeated use of the partial integration rules (6.72) or (6.74) w.r.t. $x_{i}$ into expressions, which have lower powers of $P$ in the denominator and lower homogeneous degree polynomials in $x$ in the numerators. Eventually all emergent terms can be manipulated into the form of moduli dependent functions times lower derivatives of $\Pi(z)$ w.r.t. to the moduli $z$. The relation derived in this way is one Picard-Fuchs operator. For the quintic one starts with four derivatives of $\Pi(z)$ and the emerging relation is of course the same 4th order generalized hypergeometric differential equation as in (6.64). In the multi moduli examples one has to consider various derivatives of $\Pi(z)$ w.r.t. to different combinations $z$ as starting point and the calculation becomes quite tedious. Nevertheless one can give criteria when the left ideal of differential relations is sufficient to determine $\Pi(z)$ and systematize the calculations somewhat using a Groebner basis for the ring of monomials in the $x$ [79, 80].

\subsection{Explicite solutions to the Picard-Fuchs equations}

A solution to (6.64) will correspond a priori to an arbitrary linear combination of period integrals. To understand the physical duality symmetries and the mirror map of the model it is important to find a basis of solutions which corresponds to an integral basis of $H^{3}(M, \mathbf{Z})$. This can be achieved by requiring that the monodromy group is realized by a subgroup of $\operatorname{Sp}(4, \mathbf{Z})$. In rescaled variables $z \rightarrow \tilde{z}=5^{5} z(6.64)$ has regular singular points at $\tilde{z}=0,1, \infty$. I.e. the moduli space is $\mathbf{P} \backslash\{0,1, \infty\}$ and we drop the tilde from the $z$. At $z_{0}=0$ the indical equation, i.e. the equation for $\alpha$ in solving 6.64 with a local power series ansatz $\omega(z)=\left(z-z_{0}\right)^{\alpha} \sum_{n=0} a_{n}\left(z-z_{0}\right)^{n}$ is $\alpha^{4}=0$. This degeneracy of solutions means that beside the unique power series solutions one has three logarithmic solutions. Because of the logarithms the mondromy around this point has in a suitable basis an upper triangular form with a maximal shift symmetries. Near $z_{0}=1$ the indicial equation has solutions $\{0,1,1,2\}$ and near $z_{0}=1 / z=0$ one has solutions $\{1 / 5,2 / 5,3 / 5,4 / 5\}$ for $\alpha$. The latter implies that one has an order 5 monodromy around $z=\infty$. The order two degeneration of the solutions at $z_{0}=1$ indicates three power series and one logarithmic solution. The monodromies around these special points are easily worked out. We refer to the basis 6.85), which is the canonical large radius basis of the mirror. For the quintic one has $\int c_{2} \omega=50$ and $A_{11}=\frac{11}{2}$. In the rescaled variable $z$ the monodromies are

$$
M_{0}=\left(\begin{array}{rrrr}
1 & 0 & 0 & 0 \\
1 & 1 & 0 & 0 \\
5 & -3 & 1 & -1 \\
-8 & -5 & 0 & 1
\end{array}\right), M_{1}=\left(\begin{array}{rrrr}
1 & 0 & -1 & 0 \\
0 & 1 & 0 & 0 \\
0 & 0 & 1 & 0 \\
0 & 0 & 0 & 1
\end{array}\right), M_{\infty}^{-1}=\left(\begin{array}{cccc}
-4 & 3 & -1 & 1 \\
1 & 1 & 0 & 0 \\
5 & -3 & 1 & -1 \\
8 & -1 & 0 & 1
\end{array}\right)
$$

Our notation is that monodromies which go counter clock wised are positive, see Fig. 16. One has of course the relation $M_{\infty}^{-1}=M_{1} M_{0}$. Remarkable is the monodromy $M_{0}$ around $z=0$. This is the point of maximal unipotency. A monodomy is called quasi-unipotent of index at most $k$ if here is some $N$ so that

$$
\left(T^{N}-\mathbf{1}\right)^{k+1}=0
$$




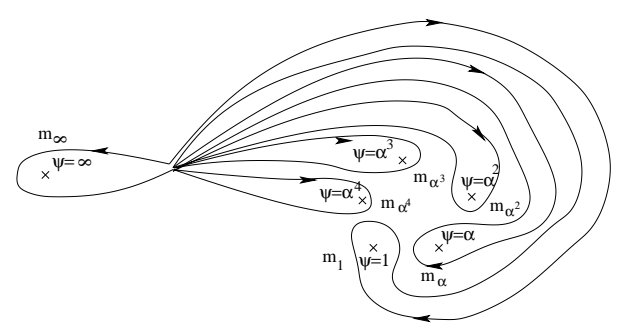

Figure 17: Quintic monodromies in the unfolded $\Psi$ modulispace

As it has been shown [107] if the period map is semi stable the monodromy is unipotent. This means $N=1$. Moreover [131] shows that the maximal $k$ that occurs as monodromy of periods is $k=\operatorname{dim}_{C}(M) . M_{0}$ saturates this bound and is of the maximal unipotency 3 . This means in particular that a solution with cubic logarithm appears at this point. As was argued in [25] discovering (6.81) is that this structure is needed to map to the large radius expansion of the mirror manifold given by (6.85). A corollary to the mirror conjecture is then that all Calabi-Yau manifolds have at least one point of maximal unipotent monodromy [116].

The monodromies in original paper [25] have been worked out in variable $\psi=z^{-\frac{1}{5}}$. This yields in the above basis

$$
m_{\infty}=\left(\begin{array}{rrrr}
-19 & 32 & -16 & 4 \\
5 & -7 & 4 & -1 \\
25 & -40 & 21 & -5 \\
-40 & 64 & -32 & 9
\end{array}\right), m_{1}=\left(\begin{array}{rrrr}
1 & 0 & -1 & 0 \\
0 & 1 & 0 & 0 \\
0 & 0 & 1 & 0 \\
0 & 0 & 0 & 1
\end{array}\right), A=\left(\begin{array}{cccc}
1 & 0 & 1 & 0 \\
-1 & 1 & -1 & 0 \\
-5 & 8 & -4 & -1 \\
3 & 5 & 3 & 1
\end{array}\right)
$$

with $m_{\alpha^{k}}=A^{-k} m_{1} A^{k}$. In the unfolded moduli space there are five copies of the conifold and encircling all five yields $m_{\infty}=M_{0}^{5}$, see Fig. 17. Monodromies for more parameter families have been investigated in [26] 88 [27].

One of the mysterious properties is the integral expansion of the mirror map at the point of maximal unipotent monodromy. We exponentiate (6.81) invert it and expand $z(q)$ in $q=e^{t}$. Call $j_{m}=\frac{1}{z(q)}$ in analogy with the normalized $j_{e}(q) S l(2, \mathbf{Z})$ invariant function of the elliptic curve. Both expansions have positive integral coefficients

$$
\begin{aligned}
& j_{e}=\frac{1}{q}+744+196884 q+21493760 q^{2}+864299970 q^{3}+20245856256 q^{4}+\ldots \\
& j_{q}=\frac{1}{q}+770+421375 q+274007500 q^{2}+236982309375 q^{3}+251719793608904 q^{4}+\ldots
\end{aligned}
$$

The integrality should be related to monodromy group $\Gamma \in \operatorname{Sp}(4, \mathbf{Z})$ generated by $M_{0}$ and $M_{1}$.

For a Calabi-Yau in an general toric ambient space one can determine the generators of the Mori cone of $M$. These are vectors, which represent curves $C^{(a)}, a=1, \ldots, h_{11}$ in the Calabi-Yau space $M$ that are dual to the Kählercone

$$
l^{(a)}=\left(l_{0,1}^{(a)}, \ldots, l_{0, r}^{(a)} ; l_{1}^{(a)}, \ldots, l_{n}^{(a)}\right), \quad \text { for } a=1, \ldots, h_{1,1} .
$$

Their first entries $l_{0,1}^{(a)}, \ldots, l_{0, r}^{(a)}$ the (multi)degree of the algebraic constraints $P_{1}=0, \ldots, P_{r}=0$ defining the Calabi-Yau manifold w.r.t to the dual divisors of $C^{(a)}$. The second entries $l_{1}^{(a)}, \ldots, l_{n}^{(a)}$ are 


\begin{tabular}{|l|r|r|}
\hline$d$ & rational & elliptic \\
\hline 1 & 2875 & 0 \\
2 & 609250 & 0 \\
3 & 317206375 & 609250 \\
4 & 242467530000 & 3721431625 \\
5 & 229305888887625 & 12129909700200 \\
6 & 248249742118022000 & 31147299733286500 \\
7 & 295091050570845659250 & 71578406022880761750 \\
8 & 375632160937476603550000 & 154990541752961568418125 \\
\hline
\end{tabular}

Table 4: BPS degeneracies $n_{\beta=d}^{(g)}$ associated to rational and elliptic curves on the Quintic in $\mathbf{P}^{4}$

the intersections of the curve $C^{(a)}$ with the toric divisors of the ambient space. The curves and the intersection numbers can be determined purely combinatorially from the toric description of the ambient space, see [80] for details. E.g. for the quintic one has $l^{(1)}=(-5 ; 1,1,1,1,1)$. With these data and the classical intersections numbers $\kappa_{a b c}=D_{a} \cap D_{b} \cap D_{c}$, which is also determined combinatorially (it is $\kappa_{111}=5$ for the quintic), one can write down a local expansion of the periods convergent near the large complex structure point, which is characterized by its maximal unipotent monodromy. We review in the following just the essentials and refer to [80] for further details. A particular set of local coordinates $z_{a}$ on the complex structure moduli space on $W$ is defined by $z_{b}=(-1)^{\sum_{a} l_{0, a}^{(b)}} \prod_{i=1}^{n} a_{i}^{l_{i}^{(b)}}, b=1, \ldots, h^{21}$ in terms of $a_{i}$, the coefficients in the polynomial constraints of the complete intersection in the torus variables 6.55. A point of maximal unipotent monodromy is then always at $z_{b}=0$. Let $\varpi_{a_{1}, \ldots, a_{s}}$ be obtained by the Frobenius method ${ }^{28}$ from the coefficients of the holomorphic function $\varpi(\vec{z}, \vec{\rho})$ defined as

$$
\begin{aligned}
\varpi\left(z_{1}, \ldots, z_{h}, \rho_{1}, \ldots, \rho_{h}\right) & =\sum_{\left\{n_{a}\right\}} c\left(n_{1} \ldots n_{h}, \rho_{1} \ldots \rho_{h}\right) \prod_{a=1}^{h} z_{a}^{n_{a}+\rho_{a}} \\
c\left(n_{1}, \ldots, n_{h}, \rho_{1}, \ldots, \rho_{h}\right) & =\frac{\prod_{m=1}^{r} \Gamma\left(1-\sum_{a=1}^{h} \hat{l}_{m}^{(a)}\left(n_{a}+\rho_{a}\right)\right)}{\prod_{i=1}^{n} \Gamma\left(1+\sum_{a=1}^{h} l_{i}^{(a)}\left(n_{a}+\rho_{a}\right)\right)} \\
\varpi_{a_{1}, \ldots, a_{s}}\left(z_{1}, \ldots, z_{h}\right) & =\left.\left(\frac{1}{2 \pi i}\right)^{s} \partial_{\rho_{a_{1}}} \ldots \partial_{\rho_{a_{s}}} \varpi\left(z_{1}, \ldots, z_{h}, \rho_{1}, \ldots, \rho_{h}\right)\right|_{\left\{\rho_{a}=0\right\}} .
\end{aligned}
$$

Define also $\sigma_{a_{1}, \ldots, a_{s}}=\left(\left.\varpi_{a_{1}, \ldots, a_{s}}\left(z_{1}, \ldots, z_{h}\right)\right|_{\log \left(z_{a}\right)=0}\right) /\left.\varpi\left(z_{1}, \ldots, z_{h}, \rho_{1}, \ldots, \rho_{h}\right)\right|_{\left\{\rho_{a}=0\right\}}$. At the large complex structure point the mirror map defines natural flat coordinates on the Kähler moduli space of the original manifold $M$

$$
t^{a}=\frac{X^{a}}{X^{0}}=\frac{1}{2 \pi i}\left(\log \left(z_{a}\right)+\sigma_{a}\right), \quad a=1, \ldots, h,
$$

where $X^{0}=\left.\varpi\left(z_{1}, \ldots, z_{h}, \rho_{1}, \ldots, \rho_{h}\right)\right|_{\rho=0}$ is the unique holomorphic period at $z_{a}=0$ and $X^{a}=\varpi_{a}$ are the logarithmic periods. Double and triple logarithmic solutions are given by [ 00

$$
w_{a}^{(2)}=\frac{1}{2} \sum_{b, c=1}^{h} \kappa_{a b c} \varpi_{b c}\left(z_{1}, \ldots, z_{h}\right), \quad a=1, \ldots, h .
$$

\footnotetext{
${ }^{28}$ The holomorphic period $\varpi\left(z_{1}, \ldots, z_{h}\right)$ can also be directly integrated using a residue expression for the holomorphic $(3,0)$ form [80].
} 


$$
w^{(3)}=\frac{1}{6} \sum_{a, b, c=1}^{h} \kappa_{a b c} \varpi_{a b c}\left(z_{1}, \ldots, z_{h}\right)
$$

where $\kappa_{a b c}$ are the classical intersection numbers $\kappa_{a b c}=D_{a} \cap D_{b} \cap D_{c}$. The prepotentials $F^{(0)}\left(X^{I}\right)$ in homogeneous or $\mathscr{F}^{(0)}\left(t^{a}\right)$ in inhomogeneous coordinates can now be written as

$$
\begin{aligned}
F^{(0)} & =-\frac{\kappa_{a b c} X^{a} X^{b} X^{c}}{3 ! X^{0}}+A_{a b} \frac{X^{a} X^{b}}{2}+c_{a} X^{a} X^{0}-i \chi \frac{\zeta(3)}{2(2 \pi)^{3}}\left(X^{0}\right)^{2}+\left(X^{0}\right)^{2} f(q) \\
& =\left(X^{0}\right)^{2} \mathscr{F}(0)=\left(X^{0}\right)^{2}\left[-\frac{\kappa_{a b c} t^{a} t^{b} t^{c}}{3 !}+A_{a b} \frac{t^{a} t^{b}}{2}+c_{a} t^{a}-i \chi \frac{\zeta(3)}{2(2 \pi)^{3}}+f(q)\right]
\end{aligned}
$$

where $q_{a}=\exp \left(2 \pi i t^{a}\right), c_{a}=\frac{1}{24} \int_{X} \operatorname{ch}_{2} J_{a}$ and $\chi$ is the Euler number of $X$. The real coefficients $A_{a b}$ are not completely fixed. They are unphysical in the sense that $K(t, \bar{t})$ and $C_{a b c}(q)$ do not depend on them. A key technical problem ${ }^{29}$ in the calculation is to invert the exponentiated mirror map (6.81) to obtain $z_{i}(\underline{t})$. An integral symplectic basis for the periods is given by

$$
\Pi=X^{0}\left(\begin{array}{c}
1 \\
t^{a} \\
2 \mathscr{F}^{(0)}-t^{a} \partial_{t^{a}} \mathscr{F}(0) \\
\partial_{t^{a}} \mathscr{F}^{(0)}
\end{array}\right)=X^{0}\left(\begin{array}{c}
1 \\
t^{a} \\
\frac{\kappa_{a b c} t^{a} t^{b} t^{c}}{3 !}+c_{a} t^{a}-i \chi \frac{\zeta(3)}{(2 \pi)^{3}}+2 f(q)-t^{a} \partial_{t^{a}} f(q) \\
-\frac{\kappa_{a b c} c^{b} t^{c}}{2}+A_{a b} t^{b}+c_{a}+\partial_{t^{a}} f(q)
\end{array}\right)
$$

This period vector can be uniquely given in terms of (6.83, 6.80) by adapting the leading log behavior. The $A_{a b}$ are further restricted by the requirement that the Peccei-Quinn symmetries $t^{a} \rightarrow t^{a}+1$ act as integral $\operatorname{Sp}\left(2 h^{11}+2, \mathbf{Z}\right)$ transformations on $\Pi$. Note that $\mathscr{F}^{(0)}$ can be read off from the periods and since $t^{a}$ are flat coordinates, we have

$$
C_{a b c}(q)=\partial_{t^{a}} \partial_{t^{b}} \partial_{t^{c}} \mathscr{F}^{(0)}=\kappa_{a b c}+\sum_{d_{a}, d_{b}, d_{c} \geq 0} n_{d}^{(0)} d_{a} d_{b} d_{c} \frac{q^{d}}{1-q^{d}},
$$

where the sum counts the contribution of the genus zero worldsheet instantons. We defined $q^{d}=$ $\prod_{a} e^{-2 \pi i d_{a} t^{a}}$ where the tuple $\left(d_{1}, \ldots, d_{h}\right)$ specifies a class $\beta$ in $H^{2}(M, \mathbf{Z})$. The expansion predicts the first column in table 4 . Higher genus predictions will be discussed in sec. 6.12 .

\subsection{Rational expressions for the threepoint couplings in generic complex structure parameters}

In the previous section we have focused on expressions of the genus 0 prepotential $\mathscr{F}$, which are expanded around the large complex structure point. The expansion parameter $q=\exp (2 \pi i)$ contains $t$, which maps in the $A$-model to the complexified area of curves in the Calabi-Yau. The phase in $t$ is so that $q \rightarrow 0$ if the real area in $t$ goes to infinity. This is the natural expansion for the Gromow-Witten invariants, where small $q$ corresponds to large areas and hence suppressed instanton corrections.

For global considerations and the calculation of the holomorphic anomaly it is useful to have expressions for the three point couplings in terms of the complex structure parameters.

\footnotetext{
${ }^{29}$ We wrote an improved code for that $[\sqrt{103}]$.
} 
One way to derive them is to start with full system of Picard-Fuchs operators $\mathscr{D}_{i} \Pi(Z)=0$, $i=1, \ldots, r$. With reference to 6.14 ) we now define

$$
\begin{aligned}
W^{\left(k_{1}, \cdots, k_{d}\right)} & =\sum_{l}\left(z^{l} \partial_{z_{1}}^{k_{1}} \cdots \partial_{z_{d}}^{k_{d}} \mathscr{F}_{l}-\mathscr{F}_{l} \partial_{z_{1}}^{k_{1}} \cdots \partial_{z_{d}}^{k_{d}} z^{l}\right) \\
& :=\sum_{l}\left(z^{l} \partial^{\mathbf{k}} \mathscr{F}_{l}-\mathscr{F}_{l} \partial^{\mathbf{k}} z^{l}\right) .
\end{aligned}
$$

In this notation, $W^{(\mathbf{k})}$ with $\sum k_{i}=3$ describes the various types of triple couplings and by (6.43) and consideration of type $W^{(\mathbf{k})} \equiv 0$ for $\sum k_{i}=0,1,2$. If we now write the Picard-Fuchs differential operators in the form

$$
\mathscr{D}_{\alpha}=\sum_{\mathbf{k}} f_{\alpha}^{(\mathbf{k})} \partial^{\mathbf{k}}
$$

then we immediately obtain the relation

$$
\sum_{\mathbf{k}} f_{\alpha}^{(\mathbf{k})} W^{(\mathbf{k})}=0
$$

Further relations are obtained from operators $\partial_{z_{i}} \mathscr{D}_{\alpha}$. If the system of PF differential equations is complete, it is sufficient for deriving linear relations among the triple couplings and their derivatives, which can be integrated to give the Yukawa couplings up to an overall normalization. In the derivation, we need to use the following relations which are easily derived

$$
\begin{aligned}
& W^{(4,0,0,0)}=2 \partial_{z_{1}} W^{(3,0,0,0)} \\
& W^{(3,1,0,0)}=\frac{3}{2} \partial_{z_{1}} W^{(2,1,0,0)}+\frac{1}{2} \partial_{z_{2}} W^{(3,0,0,0)} \\
& W^{(2,2,0,0)}=\partial_{z_{1}} W^{(1,2,0,0)}+\partial_{z_{2}} W^{(2,1,0,0)} \\
& W^{(2,1,1,0)}=\partial_{z_{1}} W^{(1,1,1,0)}+\frac{1}{2} \partial_{z_{2}} W^{(2,0,1,0)}+\frac{1}{2} \partial_{z_{3}} W^{(2,1,0,0)} \\
& W^{(1,1,1,1)}=\frac{1}{2}\left(\partial_{z_{1}} W^{(0,1,1,1)}+\partial_{z_{2}} W^{(1,0,1,1)}+\partial_{z_{3}} W^{(1,1,0,1)}+\partial_{z_{4}} W^{(1,1,1,0)}\right) .
\end{aligned}
$$

For the Picard-Fuchs equation for the quintic we get in this way

$$
C_{z z z}=\frac{5}{z^{3}\left(1-5^{5} z\right)}
$$

For the system (6.70) we get after rescaling of $a=1728 z_{1}$ and $b=4 z_{2}$ the triple couplings

$$
\begin{aligned}
& C_{a a a}=\frac{4}{a^{3} \Delta_{1}}, \quad C_{a a b}=\frac{2(1-a)}{a^{2} b \Delta_{1}}, \\
& C_{a b b}=\frac{(2 a-1)}{a b \Delta_{1} \Delta_{2}}, C_{b b b}=\frac{1+b-a(1+3 b)}{2 b^{2} \Delta_{1} \Delta^{2}},
\end{aligned}
$$

where we defined the components of the discriminant as

$$
\Delta_{1}=1-2 a-a^{2}(1-b), \quad \Delta_{2}=(1-b) .
$$

\subsection{Coupling the $B$ model to topological gravity}

We consider again the moduli space introduced in Sec. 4.2

$$
\mathscr{M}_{g}=\text { large gauge transf. } \backslash \mathscr{H}_{g} /(\text { diff } \times \text { Weyl })_{g} .
$$

with expected dimension $3 g-3$ (7.41). In the covariant quantization of string theory the metric independence of the theory, up to this finite dimensional space (4.15) we presently discuss, is 
expressed by a nilpotent BRST operator just like in (4.14). Conformal invariance is maintained for $\sigma$ models on Calabi-Yau spaces. To take advantage of this extra bonus of the $B$-model note that in a conformal fields theory $T_{\mu}^{\mu}=0$ and (4.14) splits in the following two components corresponding to $T_{z z}=T(z)$ and $T_{\bar{z} \bar{z}}=\bar{T}(z)$. Now we can borrow literally the treatment of the measure from the critical bosonic string. In the case of the bosonic string the situation is exactly as in the topological $B$-model on a Calabi-Yau 3 fold (4.32), where the ghost number is identified with the $U(1)$ axial charge of the $B$-model. The geometrical reason for this equivalence is that (7.42) and (7.43) give the same anomaly if $\operatorname{dim}_{C}(M)=3$ and $c_{1}(T M)=0$. As we saw in Sec. 4.4 the $b(z)$ and the $Q_{B R S T}$ have ghost number -1 and 1 respectively and there is a ghost number anomaly of $6 g-6=-3 \chi\left(\Sigma_{g}\right)$ on a higher genus wordsheet, which corresponds to the axial current anomaly $6 g-6=-3 \chi\left(\Sigma_{g}\right)$.We can use therefore the same measure over the complex moduli space ss in the bosonic string. From the Beltrami-Differentials $\mu^{k}=\mu_{\bar{z}}^{k z} \mathrm{~d} \bar{z} \partial_{z}, k=1, \ldots, 3 g-3$ in $H^{1}(T \Sigma)$, which represent tangent directions of $\mathscr{M}_{g}$, we define

$$
B^{k}:=\int_{\Sigma_{g}} \sqrt{h} h^{\alpha \gamma} h^{\beta \delta} \delta^{(k)} h_{\alpha \beta} G_{\gamma \delta}=\int_{\Sigma_{g}} \mathrm{~d}^{2} z\left(G_{z z} \mu_{\bar{z}}^{k z}+G_{\bar{z} \bar{z}} \bar{\mu}_{z}^{k} \bar{z}\right)=\beta^{k}+\bar{\beta}^{k}
$$

The definition of $B^{(k)}$ in itself does not require conformal invariance but just (4.14). We used after the second equality the standard metric in a conformal gauge and the expressions for the BeltramiDifferentials. In the last equality we used $(2,2)$ supersymmetry and the fact that $G^{-}, \bar{G}^{-}$are $h=2$ fields after the $B$-twist to define

$$
\beta^{k}=\int_{\Sigma_{g}} \mathrm{~d}^{2} z G^{-} \mu^{k}, \quad \bar{\beta}^{k}=\int_{\Sigma_{g}} \mathrm{~d}^{2} z \bar{G}^{-} \bar{\mu}^{k} .
$$

Because of the antisymmetry of $G$ and the Kähler structure on the moduli space $\mathscr{M}_{g}$ the quantity

$$
\mu_{g}=\left\langle\prod_{k=1}^{6 g-6} B^{k}\right\rangle \cdot[\mathrm{d} M]=\left\langle\prod_{k=1}^{3 g-3} \beta^{k} \bar{\beta}^{k}\right\rangle \cdot[\mathrm{d} m \wedge \mathrm{d} \bar{m}]
$$

is a top-form on $\mathscr{M}_{g}$. Here $\cdot[\mathrm{d} M]$ or $\cdot[\mathrm{d} m \wedge \mathrm{d} \bar{m}]$ means contraction with $\mathrm{d} M_{i_{1}} \wedge \ldots \wedge \mathrm{d} M_{i_{6 g-g}}$ or $\mathrm{d} m_{i_{1}} \wedge \mathrm{d} \bar{m}_{i_{1}} \wedge \ldots \wedge \mathrm{d} m_{i_{3 g-3}} \wedge \mathrm{d} \bar{m}_{i_{3 g-3}}$ and suitable normalization. That is we inserted $6 g-6$ times $\beta^{(k)}$ to compensate the ghost or axial anomaly, which is by the index theorems (cff section7.3) identified with the dimension of $\mathscr{M}_{g}$. The integral

$$
\mathscr{F}^{(g)}=\int_{\mathscr{M}_{g}} \mu_{g}
$$

is the central observable of the topological $B$ model. How does this discussion of the dimension of the moduli space relate to (5.39). In the A-model we counted the geometrical virtual dimension of the moduli space of non-trivial maps and found that the deformations of the metric $\mathscr{M}_{g}$ are offset by the obstructions of having a nontrivial holomorphic map to $M$, so that the virtual dimension of the moduli space of maps is zero. Here we kill the deformation space of $\mathscr{M}_{g}$ by viewing the $B$-model fields as ghost system from which we construct a top form to integrate over $\mathscr{M}_{g}$. The topological $B$-model is one of those examples of string theories, where general covariance (4.14) is maintained by an $Q_{B R S T}$ operator, whose charge violation measure the dimension of the moduli space, but the decoupling of ghost and matter sector is not imposed [148]. 
As part of the prerequisite for coupling topological theories to gravity [151] the measure $\mu_{g}$ must be closed $\mathrm{d} \mu_{g}=0$. To see that consider

$$
0=\left\langle\left\{Q, \prod_{k=1}^{6 g-5} B^{k}\right\}\right\rangle=\sum_{j=1}^{6 g-5}(-1)^{j-1}\left\langle B^{1} \ldots\left\{Q, B^{j}\right\}, \ldots B^{5 g-5}\right\rangle
$$

and use the fact that $\left\{Q, B^{i}\right\}$ yields the $T^{i}=\int_{\Sigma_{g}} \mathrm{~d}^{2} z T \mu^{i}$, whose insertions can be interpreted as derivative on $\mathscr{M}_{g}$ according to 4.17 . A second prerequisite is that $\mu_{g}$ is basic, i.e. that it vanishes for all variations of the metric induced by infinitesimal diffeomorphism. These correspond to the last two terms in 4.16 and the property is easily checked. We will show below explicitly by manipulations similar to the one that lead to 6.98) that the $Q$ commutator of the measure is exact. The metric dependence comes from the boundaries of $\mathscr{M}_{\mathrm{g}}$. Combinatorially the calculation is like non-topological higher string loop calculations, apart from the much more sophisticated integrals over $\mathscr{M}_{g}$. The compactifications of $\mathscr{M}_{g, n}$ is identical to the one discussed in Sec. 5.2. Its boundary components come from pairwise collision of inserted points and nodes. In $2 d$ gravity we got from these boundaries the topological recursion relations. In the case of the $B$-model there is an interesting modification namely that the boundary components contribute only in anti-holomorphic derivatives of $\mathscr{F}_{g}$, which gives rise to recursion relations involving antiholomorphic derivatives. Since without boundary component contributions the $\mathscr{F}^{(g)}$ would be holomorhic one calls these recursions the holomorphic anomaly equations. They are no more anomalous then the topological recursion relations.

\subsection{The holomorphic anomaly}

We want to consider in this section perturbations of a more general form then in Sec. 4.3 namely

$$
S=\int_{\Sigma} \mathrm{d}^{2} z \mathscr{L}_{0}+\sum_{i} t^{i} \int_{\Sigma} \mathscr{O}_{i}+\sum_{i} \bar{t}^{i} \int_{\Sigma} \overline{\mathscr{O}}_{i}
$$

Here the WS two-form field $\mathscr{O}=\mathscr{O}^{(2)}$ is the B-model field (4.12) which comes from a $\phi=\mathscr{O}^{(0)}$ in the $(c, c)$ ring. We will use here the CFT notation introduced in Sec. 4.5, i.e. $\mathscr{O}_{i}:=\left\{Q_{+},\left[Q_{-}, \phi_{i}\right]\right\} \sim$ $\left\{G_{0}^{-},\left[\bar{G}_{0}^{-}, \phi_{i}\right]\right\}$ and $\overline{\mathscr{O}}_{\bar{\imath}}:=\left\{\bar{Q}_{+},\left[\bar{Q}_{-}, \bar{\phi}_{\bar{l}}\right]\right\} \sim\left\{G_{0}^{+},\left[\bar{G}_{0}^{+}, \bar{\phi}_{\bar{l}}\right]\right\}$. In a unitary theory $\bar{t}^{i}=\left(t^{i}\right)^{*}$, but it will be important in the following to view $\bar{t}^{i}$ as an independent parameter. As explained in Sec. 4.3 the WS two-form fields in (6.99) are neutral. Therefore we can expect that arbitrary $n$ - point functions like for $g>1$

$$
C_{i_{1}, \ldots, i_{n}}^{(g)}=\int_{\mathscr{M}_{g}}\left\langle\int \mathscr{O}_{i_{1}} \ldots \int \mathscr{O}_{i_{n}} \prod_{k=1}^{3 g-3} \beta^{k} \bar{\beta}^{k}\right\rangle
$$

do not vanish. As it stands (6.100) is not well defined. We first have to specify how to deal with the contact terms, which are necessarily present in an interacting supersymmetric theory, see (4.64) or (4.71). Now in the case $g=0$ there are the three $\operatorname{PSL}(2, \mathbf{C})$ conformal Killing fields. The zero mode integral of their superpartners compensates for three descendant operations and with the $\operatorname{PSL}(2, \mathbf{C})$ symmetry we set three points to $0,1, \infty$. The generic genus zero correlation is then

$$
C_{i_{1}, \ldots, i_{n}}^{(0)}=\int_{\mathscr{M}_{0}}\left\langle\phi_{i_{1}}(0) \phi_{i_{2}}(1) \phi_{i_{3}}(\infty) \int \mathscr{O}_{i_{4}} \ldots \int \mathscr{O}_{i_{n}}\right\rangle
$$


This has no contact interaction among the first 3 fields. It is natural to make this function symmetric in its indices. Therefore we exclude all contact interactions from the regions of the integrations. This is the regularization we adopt for general $g$.

In view of (6.99) we can insert $\int_{\Sigma} \mathscr{O}_{i}$ operators by taking $t^{i}$ derivatives $\partial_{i}$ of $C_{i_{1}, \ldots, i_{n}}^{(g)}$ in an the attempt to obtain $C_{i, i_{1}, \ldots, i_{n}}^{(g)}$. In order to achieve our short distance regularization we have to subtract the would be contact terms in the integration over $\Sigma$. This is very naturally achieved by taking covariant derivatives w.r.t. the Weil-Peterson metric, i.e. $\partial_{i} \rightarrow \partial_{i}-\Gamma_{i}$. In the $t t^{*}$ formalism we can isolate the contact term as the difference between $\partial_{i}\left(Q_{+} Q_{-}|j\rangle\right)-\mathscr{O}_{j} \partial_{i}|0\rangle=\left[\left(A_{i}\right)^{k}\right)_{j} \mathscr{O}_{k}-$ $\left.\left(A_{i}\right)_{0}^{0} \mathscr{O}_{j}\right]|0\rangle$. The logic is that in the term $\partial_{i}\left(Q_{+} Q_{-}|j\rangle\right)$ the field $\mathscr{O}_{i}$ in the integral $\int_{\Sigma} \mathscr{O}_{i}$ explores the region near $\mathscr{O}_{j}$ in 4.47 , while in the second it does not. The $Q_{+} Q_{-}$generate the descendant field from $\phi_{j}$ in (4.47) in order to compare the two terms. In particular applying this to $|j\rangle=|0\rangle$ and using (4.68,4.69) we get a contact term with the $\mathbf{1}$ operator $\left(A_{i}\right)_{0}^{0} \cdot \mathbf{1}=-\partial K \cdot \mathbf{1}$. Roughly speaking this non triviality of the vacuum comes from the coupling of $\phi_{j}$ to the $U(1)_{R}$ current (4.2). One can argue that the above contact term is proportional to the integral of $R$ integrated over the Riemann surface. The above consideration for the half sphere (4.47), fixes the normalization and in general gives the Euler number $\chi$ of $\Sigma$. Subtracting both contact terms one concludes that the insertion of $\int_{\Sigma} \mathscr{O}_{i}^{(2)}$ into a genus $g$ correlation function with the right short distance prescription is given by the covariant derivative of $C_{i_{1}, \ldots, i_{n}}^{(g)}$

$$
D_{i}=\partial_{i}-\Gamma_{i}-(2-2 g) \partial_{i} K,
$$

This reflects the fact that $C_{i_{1}, \ldots, i_{n}}^{(g)}$ is a tensor over the complex moduli space of the Calabi-Yau $\mathscr{M}$ transforming in $\operatorname{Sym}^{n}\left(T^{*} \mathscr{M}\right) \otimes \mathscr{L}^{2-2 g}$ in as a generalization of the genus zero discussion in Sec. 6.6. The last factor can also be understood by building the higher genus Riemann surface $\Sigma_{g}$ by sewing it from a sphere. This involves $g$ times a $|i\rangle \eta^{i j}\langle j| \in \mathscr{L}^{-2}$ insertion as we will see shortly, which results in $\mathscr{F}^{(g)}$ transforming as section of $\mathscr{L}^{2-2 g}$ w.r.t. to Kähler transformations. To summarize the contact algebra analysis yields that all correlators can be obtained from the vacuum correlators $\mathscr{F}^{g}$ as

$$
C_{i_{1}, \ldots, i_{n}}^{(g)}=D_{i 1} \ldots D_{i_{n}} \mathscr{F}^{(g)} .
$$

They are symmetric, because of the vanishing of the corresponding curvature terms in Kähler connections.

Let us therefore investigate similarly as in Sec. (4.62) the derivative w.r.t. $\bar{t}_{i}$ of the correlator

$$
\begin{aligned}
\frac{\partial}{\partial \bar{t}_{i}} \mathscr{F}^{g} & =\int_{\mathscr{M}_{g}}\left\langle\oint_{C_{w}} G^{+} \oint_{C_{w}^{\prime}} \bar{G}^{+} \bar{\phi}_{\bar{i}}(w) \prod_{k, \bar{k}=1}^{3 g-3} \beta^{k} \beta^{\bar{k}}\right\rangle \cdot[\mathrm{d} m \wedge \mathrm{d} \bar{m}] \\
& =\int_{\mathscr{M}_{g}} 4 \sum_{i \bar{l}=1}^{3 g-3} \frac{\partial^{2}}{\partial m_{i} \partial \bar{m}_{i}}\left\langle\phi_{\bar{i}}(w) \prod_{k \neq i} \beta^{k} \prod_{\bar{k} \neq \bar{l}} \beta^{\bar{k}}\right\rangle \cdot[\mathrm{d} m \wedge \mathrm{d} \bar{m}] \\
& =\int_{\mathscr{M}_{g}} \partial \bar{\partial} \lambda^{6 g-8}=\int_{\partial \mathscr{M}_{g}} \lambda^{6 g-8}
\end{aligned}
$$

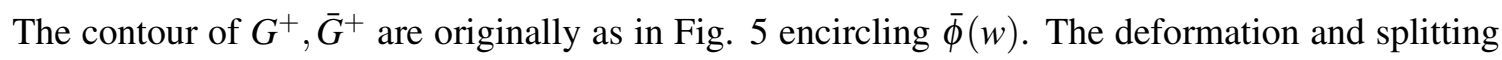
of the contour yields a sum of terms in which the $G^{+}$and $\bar{G}^{+}$encircle one $\oint_{C_{u}} \mathrm{~d} w G^{+}(w) G^{-}(u) \mu^{k}=$ $2 T(u) \mu^{k}$ and one $\oint_{C_{u}} \mathrm{~d} w \bar{G}^{+}(w) \bar{G}^{-}(u) \mu^{\bar{k}}=2 \bar{T}(u) \mu^{\bar{k}}$ in each summand. Together with the integral 


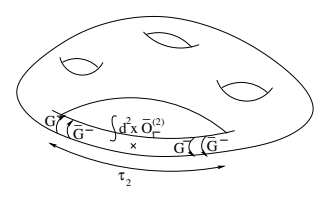

Figure 18: A-type sewing

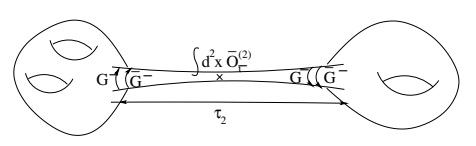

Figure 19: B-type sewing

in the definition of the $\beta^{k}$ and $\bar{\beta}^{k}$ and the charges $Q_{+}$and $Q_{-}$associated to $G^{+}(z)$ and $\bar{G}^{+}(z)$ we can write the result of the contour deformation as

$$
\begin{aligned}
& \left\{Q_{-}, \beta^{k}\right\}=\int_{\Sigma_{g}} \mathrm{~d}^{2} z T \mu^{k}=: T^{k} \\
& \left\{Q_{+}, \bar{\beta}^{k}\right\}=\int_{\Sigma_{g}} \mathrm{~d}^{2} z \bar{T} \bar{\mu}^{k}=: \bar{T}^{k} .
\end{aligned}
$$

In Sec. 4.5 where the $G^{-}(u), \bar{G}^{-}(u)$ are integrated over a contour we got the $L_{-1}$ mode of the $T$, which corresponds to derivative of an insertion position. Here we get the $T^{k}$ and $\bar{T}^{k}$, which convert according to 4.17 ) into a derivative in the moduli space. Both effects are related and lead to exact forms on $\mathscr{M}_{g}$ and $\mathscr{M}_{g, n}$. The boundary components $\partial \mathscr{M}_{g}$, where the integral in the last line of (6.104) contributes according to Cauchy's theorem are in real codimension two as indicated by the form degree of $\lambda$. They are the standard stable degenerations encountered in Sec 5.2 Fig 10. The whole point specific of the $B$-model is to now figure out what the $P_{i j}, A_{i j}$ and $B_{i j}$ are. This turns out to be much easier then in the $2 d$ gravity case. It is a bosonic string higher loop sewing consideration [126] with simplifications. There will be no new information in the $P_{i j}$ above what we summarized in (6.103). Since $\int_{\Sigma} \mathscr{O}^{(i)}$ operators correspond to functions on $\mathscr{M}_{g}$ as opposed to the $\Psi$ classes there is no interesting recursion to expect.

It remains to analyze the $A$ and $B$ degeneration depicted in Fig. 18 and 19 respectively. Near the boundary component in the moduli space corresponding to the degenerate surface in the figures the normal direction to the boundary can be parametrized by the length of the tube $\tau_{2}$. The moduli space of the boundary components consist of the $3 g-6$ dimensional moduli space of the irreducible curves of genus $g-1$ in case A or $h$ and $g-h$ in case B respectively with measure $[\mathrm{d} \hat{m} \wedge \mathrm{d} \hat{\bar{m}}]$. That is we loose three complex dimensions in the moduli space of the irreducible components and hence three $\beta \bar{\beta}$. As we make the tube infinitely long or equivalently infinitesimally thin the data remembered about the shape are merely the two insertion points $w$ and $u$, the length and the twist of the tube. In particular two $\beta \bar{\beta}$ are replaced by $\left(\oint_{C_{x}} G^{-} \oint_{C_{x}^{\prime}} G^{-} \phi_{X}(x)\right)$ with $x=u, w$ and since we want to calculate a string amplitude we have to insert a complete set of states for the $\phi_{X}$. The contribution of the boundary is hence

$$
\int_{\partial M_{g}}[\hat{\mathrm{d}} m \wedge \hat{\mathrm{d}} \bar{m}][d w][d u] \frac{\partial}{\partial \tau_{2}}\left\langle\int \bar{\phi}_{\bar{j}}\left(\oint_{C_{u}} G^{-} \oint_{C_{u}^{\prime}} G^{-} \phi_{i}\right) \eta^{i j}\left(\oint_{C_{w}} G^{-} \oint_{C_{w}^{\prime}} G^{-} \phi_{j}\right) \prod_{a=1}^{3 g-6} \hat{\beta}^{a} \hat{\bar{\beta}}^{a}\right\rangle
$$

The integration over $[d u]$ and $[d w]$ is over the fibre $\Sigma_{g}$ of the universal curve. We can hence convert, e.g. the $\oint_{C_{u}} G^{-} \oint_{C_{u}^{\prime}} G^{-} \phi_{i}$ insertions in a descendant field $\mathscr{O}_{j}^{(2)}$ integrated over $\Sigma_{g}$. Only if the $\int \bar{\phi}_{\bar{j}}$ 
integral extends over the tube one gets a contribution proportional to $\tau_{2}$ which does not cancel under the derivative in 6.106 and one can focus on this integration domain. The correlation function factorizes upon complete insertion of states in operator approach, which gives

$$
\int_{\partial M_{g}}[\mathrm{~d} \hat{m} \wedge \mathrm{d} \hat{\bar{m}}] \frac{\partial}{\partial \tau_{2}}\left\langle k\left|\int_{t u b e} \bar{\phi}_{\bar{j}}\right| l\right\rangle \eta^{i k} \eta^{l j}\left\langle\left(\int_{\Sigma} \mathscr{O}_{i}\right)\left(\int_{\Sigma} \mathscr{O}_{j}\right) \prod_{a=1}^{3 g-6} \hat{\beta}^{a} \hat{\bar{\beta}}^{a}\right\rangle .
$$

Here we also used the fact that propagation on the tube projects on the ground state. With the manipulations from the Sec. 4.5 and the normalizing the perimeter of the tube to one we get

$$
\begin{aligned}
\left\langle k\left|\int_{\text {tube }} \bar{\phi}_{\bar{j}}\right| l\right\rangle \eta^{i k} \eta^{l j} & =\left\langle\bar{k}\left|\int_{\text {tube }} \bar{\phi}_{\bar{j}}\right| \bar{l}\right\rangle M_{k}^{\bar{k}} \eta^{i k} M_{l}^{\bar{l}} \eta^{l j} \\
& =\tau_{2}\left\langle\bar{k}\left|\bar{\phi}_{\bar{j}}\right| \bar{l}\right\rangle e^{2 K} G^{i \bar{k}} G^{j \bar{l}}=\tau_{2} \bar{C}_{\bar{k} \bar{j} l} e^{2 K} G^{i \bar{k}} G^{j \bar{l}}=: \tau_{2} C_{\bar{k}}^{i j}
\end{aligned}
$$

Using this result in the boundary contribution of the $A$ or $B$ type degeneration and 6.103 one gets the contributions from the boundaries

$$
\bar{\partial}_{\bar{k}^{\prime}} \mathscr{F}^{(g)}=\frac{1}{2} \bar{C}_{\bar{k}}^{i j}\left(D_{i} D_{j} \mathscr{F}^{(g-1))}+\sum_{r=1}^{g-1} D_{i} \mathscr{F}^{(r)} D_{j} \mathscr{F}^{(g-r)}\right)
$$

The factor $\frac{1}{2}$ comes from the fact that we over count the integration over $\mathscr{O}_{i}$ and $\mathscr{O}_{j}$ in (6.107) by two in the $A$ degeneration, as the $\mathscr{O}_{i} \leftrightarrow \mathscr{O}_{j}$ does not change the complex structure and in the $B$ degeneration we doubled the non symmetric terms.

For $g=1$ the situation is more tricky and interesting. Because of $h^{0}\left(T^{2}\right)=1$ we have to kill the infinite automorphism by the insertion of one operator to start with a stable curve. Hence we have to consider $\bar{\partial}_{\bar{k}} \partial_{m} \mathscr{F}^{(1)}$. That leads in addition to the $A$ degeneration to a contact term between $\mathscr{O}_{i} \overline{\mathscr{O}}_{\bar{j}}$

$$
\bar{\partial}_{\bar{k}} \partial_{m} \mathscr{F}^{(1)}=\frac{1}{2} \bar{C}_{\bar{k}}^{i j} C_{m i j}+\left(\frac{\chi}{24}-1\right) G_{\bar{k} m} .
$$

The first term above is from the $A$ type degeneration. The contact term sees global properties of the Calabi-Yau and is the most interesting one have encountered. There are two ways to normalize the contact term. Compare with the operator

$$
\mathscr{F}_{1}(t, \bar{t})=\frac{1}{2} \int \frac{\mathrm{d}^{2}}{\tau_{2}} \operatorname{Tr}(-1)^{F} F_{L} F_{R} q^{H} \bar{q}^{\bar{H}} .
$$

formulation $\mathscr{F}^{(1)}[10]$ and calculate the $t \bar{t}$ term as in [30]. Or relate it to the Ray-Singer torsion ${ }^{30}$ [11] and use the family index theorem of [14].

The counting function for the GW invariants is obtained as a holomorphic limit of the result of the integration $\mathscr{F}^{\text {top }}(t)=\lim _{\bar{t} \rightarrow \infty} \mathscr{F}^{g}(t, \bar{t})$ of $(6.110)$. One difficulty in integrating $\mathscr{F}^{g}(t, \bar{t})$ is the possibility of adding a holomorphic piece to it. Its form is however restricted to

$$
f_{g}(z)=\sum_{i=1}^{D} \sum_{k=0}^{2 g-2} \frac{p_{i}^{(k)}(z)}{\Delta_{i}^{k}}
$$

where $D$ is the number of components $\Delta_{i}$ of the discriminant, and $p_{i}^{(k)}(z)$ are polynomials of degree $k$. Using the expansion (5.48) and the genus one data of the quintic discussed in (6.9) one obtains the BPS numbers in table 7 and .

\footnotetext{
${ }^{30}$ See [125] for a recent application to Hitchins action.
} 


\begin{tabular}{|l|r|r|r|}
\hline$d$ & arith. genus 2 & 3 & 4 \\
\hline 1 & 0 & 0 & 0 \\
2 & 0 & 0 & 0 \\
3 & 0 & 0 & 0 \\
4 & 534750 & 8625 & 0 \\
5 & 75478987900 & -15663750 & 15520 \\
6 & 871708139638250 & 3156446162875 & -7845381850 \\
7 & 5185462556617269625 & 111468926053022750 & 243680873841500 \\
8 & 22516841063105917766750 & 1303464598408583455000 & 25509502355913526750 \\
\hline
\end{tabular}

Table 5: BPS degeneracies $n_{\beta=d}^{(g)}$ associated to genus 2,3,4 curves on the Quintic in $\mathbf{P}^{4}$

\section{Complex-, Kähler- and Calabi-Yau manifolds.}

Let us describe in the following the definitions and key properties of the manifolds mentioned above. A quick introduction from the physics point of view is [82], a more extensive one is [21]. A good introduction of supersymmetric compactifications with emphasis on Calabi-Yau manifolds and orbifolds is [54]. One purpose of this section is to give a guide to further mathematical references which are given as we go along.

\subsection{Complex manifolds}

Consider a real $2 n$ dimensional manifold $M$ with a covering by coordinate patches $\mathscr{U}_{i}, i=$ $1, \ldots, r$, which are homeomorphic to a neighborhood $U_{i} \in \mathbf{C}^{n}$. Then we can pick $x_{\alpha}^{(i)}(p), \alpha=$ $1, \ldots, n$ complex coordinates on each $\mathscr{U}_{i} . M$ is a complex manifold, if all transition functions

$$
f^{(j k)}: x^{(k)}(p) \rightarrow x^{(j)}(p)
$$

defined for $p \in \mathscr{U}_{j} \cap \mathscr{U}_{k}$, are biholomorphic.

Obviously $\mathbf{C}^{n}$ is a non-compact complex manifold with one chart. It is also Kähler. One may hope to get examples of compact complex manifolds by considering constraints like $f\left(x_{1}, \ldots, x_{n}\right)=$ 0 , which are holomorphic in all variables. While this leads indeed to a complex manifold, it fails to define compact ones, because of the maximum modulus theorem, which states that the maximum value of the modulus of a non constant differential function on an arbitrary domain $D$ is taken at the boundary of $D$. If now $f=0$ is solved for some $x_{i}$ in a compact domain $D$ of the other variables, $x_{i}$ takes its maximal modulus on the boundary of $D$ and the construction fails to define a differentiable compact manifold.

A way out is to use identifications on $\mathbf{R}^{2 n}$ by discrete shift symmetries, i.e. consider tori $T^{2 n}=\mathbf{R}^{2 n} / \Gamma_{2 n}$, where the lattice $\Gamma_{2 n} \cong \mathbf{Z}^{2 n}$ as abelian groups. If one chooses a complex structure on $\mathbf{R}^{2 n}$ by aligning real and imaginary directions of $T^{*} \mathbf{R}^{2 n} \cong \mathbf{R}^{2 n}$ with the basis of $\Gamma_{2 n}$ one gets compact complex tori $T_{\mathbf{C}}^{3}$. They are flat and have hence trivial holonomy. Dividing by discrete rotations $G$ of the lattice $\Gamma_{2 n}$ leads to orbifold compactifications. If $G$ acts as a discrete irreducible subgroup of $S U(3)$ in the fundamental representation on the complex coordinates of $T_{\mathbf{C}}^{3}$ then one gets a complex orbifold with curvature singularities at the fix set of $G$. The corresponding lattice 
automorphisms have been classified [50]. Remarkably one can prove that this curvature singularities can be smoothed to get a Kähler manifold with $S U(3)$ holonomy.

An alternative route to construct simple compact complex manifolds is by dividing by $\mathbf{C}^{*}:=$ $\mathbf{C} \backslash\{0\}$ actions. E.g. $\mathbf{P}^{n}$ is defined as the space of complex lines through the origin in $\mathbf{C}^{n+1}$. This is the space of equivalence classes of $\left[x_{0}, \ldots, x_{n}\right]$ in $\mathbf{C}^{n+1} \backslash\{\underline{0}\}$ with the equivalence relation

$$
\left(x_{0}, \ldots, x_{n}\right) \sim \lambda\left(x_{0}, \ldots, x_{n}\right),
$$

where $\lambda \in \mathbf{C}^{*}$. For the charts we take

$$
\mathscr{U}_{i}=\left\{x_{i} \neq 0 \mid x_{i} \in \mathbf{P}^{n}\right\}
$$

and as their coordinates $x_{m}^{(i)}=x_{m} / x_{i}$. On $\mathscr{U}_{j} \cap \mathscr{U}_{k}$ we have the transition functions

$$
x_{m}^{(i)}=\frac{x_{m}}{x_{k}} / \frac{x_{i}}{x_{k}}=\frac{x_{m}^{(k)}}{x_{i}^{(k)}}
$$

which are biholomorphic. $\mathbf{P}^{n}$ is a obviously compact and a Kähler manifold as we shall see.

A hypersurface constraint in $\mathbf{P}^{n}$ of the type $f\left(x_{0}, \ldots, x_{n}\right)=0$ must be homogeneous of some degree $d$ in the $x_{i}$, i.e. $f\left(\lambda x_{1}, \ldots, \lambda x_{n}\right)=\lambda^{d} f\left(x_{1}, \ldots, f_{d}\right)$, to be well defined on the equivalence classes. It defines a compact complex Kähler manifold. This manifold is smooth if $f$ is transversal, i.e. $\mathrm{d} f \neq 0$ for $f=0$. We will give a short overview about the application of this construction and generalizations to Calabi-Yau manifolds in Sec. 7.7.

Conceptually it is an important question if and how many complex structures an even dimension real manifold possesses. A necessary prerequisite to have a complex structure is a differentiable endomorphism of the tangent bundle $J: T M \rightarrow T M$ with $J^{2}=-\mathbf{1}$. $J$ corresponds to multiplication of the tangent bundle by $i=\sqrt{-1}$ and manifold with this structure is called an almost complex manifold ${ }^{31}$. With $J$ we can define projectors

$$
P=\frac{1}{2}(\mathbf{1}-i J)
$$

on the holomorphic sub-bundle and the antihomlomophic sub-bundle of the tangent bundle

$$
\bar{P}=\frac{1}{2}(\mathbf{1}+i J)
$$

respectively. A necessary and sufficient condition for the existence of complex coordinates, i.e. a complex structure, is that the Lie bracket (6.21) of two holomorphic vector fields $X, Y$ is always a holomorphic vector field [120] (see [76] and [21] Chap. V. for physicists review). Written with the projectors one formulates this condition as

$$
\bar{P}[P X, P Y]=0 .
$$

\footnotetext{
${ }^{31} \mathrm{~A}$ complex manifold is almost complex, because multiplying the basis of $T M$ of a complex manifold with coordinates $x^{k}=u^{k}+i w^{k}$ by $i=\sqrt{-1}$ maps $\left(\begin{array}{c}\frac{\partial}{\partial u^{k}} \\ \frac{\partial}{\partial v^{k}}\end{array}\right) \mapsto\left(\begin{array}{c}\frac{\partial}{\partial v^{k}} \\ -\frac{\partial}{\partial u^{k}}\end{array}\right)$, i.e. $J=\mathrm{d} u^{i} \otimes \frac{\partial}{\partial v^{i}}-\mathrm{d} v^{i} \otimes \frac{\partial}{\partial u^{i}}$. In holomorphic and anti-holomorphic coordinates this means $J_{j}^{i}=i \delta_{j}^{i}, J_{\bar{j}}^{\bar{l}}=-i \delta_{\bar{j}}^{\bar{l}}$ and $J_{\bar{j}}^{i}=J_{j}^{\bar{l}}=0$
} 
This integrability condition leads to $[J X, J Y]-J[X, J Y]-J[J X, Y]-[X, Y]=0$. In local flat coordinates $J(\partial b)=J_{b}^{e} \partial_{e}$ and with $J_{c}^{b} J_{d}^{c}=-\delta_{d}^{b}$, i.e. $\left(\partial_{a} J_{c}^{b}\right) J_{d}^{c}=-J_{c}^{b}\left(\partial_{a} J_{d}^{c}\right)$, this means that the so called Nijenhuis tensor vanishes identically [120]

$$
N_{b d}^{c}:=J_{b}^{a}\left(\partial_{a} J_{d}^{c}-\partial_{d} J_{a}^{c}\right)-J_{d}^{a}\left(\partial_{a} J_{b}^{c}-\partial_{b} J_{a}^{c}\right) \equiv 0
$$

Once complex coordinates $x^{k}=u^{k}+i v^{k}$ with

$$
\partial_{k}:=\frac{\partial}{\partial x^{k}}=\frac{1}{2}\left(\frac{\partial}{\partial u^{k}}-i \frac{\partial}{\partial v^{k}}\right), \quad \partial_{\bar{k}}:=\frac{\partial}{\partial \bar{x}^{k}}=\frac{1}{2}\left(\frac{\partial}{\partial u^{k}}+i \frac{\partial}{\partial v^{k}}\right)
$$

are defined, we can split $T_{\mathbf{C}} M=T_{\mathbf{R}} M \otimes \mathbf{C}$, which is spanned over $\frac{\partial}{\partial w_{k}}, k=1, \ldots, 2 n$ with complex coefficients $v^{i}$ as $T_{\mathbf{C}} M=T^{1,0} M \oplus T^{0,1} M$. Here $\left\{u_{k}, v_{k}\right\}=:\left\{w_{k}, w_{k+n}\right\}$ and each vector $V$ in $T_{\mathbf{C}} M$ decomposes as

$$
V=\sum_{k=1}^{2 n} V^{k} \frac{\partial}{\partial w_{k}}=\sum_{k=1}^{n}\left[\left(V^{k}+i V^{n+k}\right) \partial_{k}+\left(V^{k}-i V^{n+k}\right) \partial_{\bar{k}}\right]=: V^{1,0}+V^{0,1}
$$

The transition functions of $T^{1,0} M\left[T^{0,1}\right]$ spanned by $\partial_{k},\left[\partial_{\bar{k}}\right]$ are [anti-]holomorphic, and we call it the [anti]holomorphic tangent bundle. Obviously under complex conjugation $T^{0,1} M=\overline{T^{1,0} M}$. Similarly the cotangent bundle splits $T_{\mathbf{C}}^{*} M=T^{* 1,0} M \oplus T^{* 0,1} M$ into a holomorphic and an antiholomorphic sub bundle spanned by $\mathrm{d} x^{k}$ and $\mathrm{d} \bar{x}^{k}:=\mathrm{d} x^{\bar{k}}$ respectively. Sections of $\wedge^{r} T_{\mathbf{C}}^{*} M$ are called $r$-forms $\Omega^{r}$ and can be decomposed into sections of $\wedge^{p} T^{* 1,0} M \wedge^{q} T^{* 0,1}$, which are called $(p, q)$ forms $\Omega^{p, q}$, i.e the space $A^{r}$ of $r$ forms splits into the space $A^{p, q}$ of $(p, q)$-forms $A^{r}=\bigoplus_{r=p+q} A^{p, q}$. If $J$ is integrable ${ }^{32}$, the de Rham exterior derivative splits likewise into

$$
\mathrm{d}=\partial+\bar{\partial}
$$

i.e. for $\omega=\omega_{i_{1}, \ldots, i_{p}, \bar{j}_{1} \ldots, j_{q}} \mathrm{~d} x^{i_{1}} \wedge \ldots \wedge \mathrm{d} x^{i_{p}} \mathrm{~d} x^{\bar{j}} \wedge \ldots \wedge \mathrm{d} x^{\bar{j}_{q}} \in A^{p, q}$ one has

$$
\begin{aligned}
\partial \omega & =\left(\partial_{k} \omega_{i_{1}, \ldots, i_{p}, \bar{j}_{1} \ldots, \bar{j}_{q}}\right) \mathrm{d} x^{k} \wedge \mathrm{d} x^{i_{1}} \wedge \ldots \wedge \mathrm{d} x^{i_{p}} \wedge \mathrm{d} x^{\bar{j}} \wedge \ldots \wedge \mathrm{d} x^{\bar{j}_{q}} \in A^{p+1, q} \\
\bar{\partial} \omega & =\left(\partial_{\bar{k}} \omega_{i_{1}, \ldots, i_{p}, \bar{j}_{1} \ldots, \bar{j}_{q}}\right) \mathrm{d} x^{\bar{k}} \wedge \mathrm{d} x^{i_{1}} \wedge \ldots \wedge \mathrm{d} x^{i_{p}} \wedge \mathrm{d} x^{\bar{j}} \wedge \ldots \wedge \mathrm{d} x^{\bar{j}_{q}} \in A^{p, q+1}
\end{aligned}
$$

so that $\mathrm{d} \Omega^{p, q} \in A^{p+1, q} \oplus A^{p, q+1}$. It follows by consideration of the $(p, q)$ type that the equation $\mathrm{d}^{2}=0$ on $A^{*}$ implies $\partial^{2}=0, \bar{\partial}^{2}=0$ and $\bar{\partial} \partial+\partial \bar{\partial}=0$. Since $\bar{\partial}$ is nilpotent we can define the cohomology $H_{\bar{\partial}}^{*}=\frac{\operatorname{Kern} \bar{\partial}}{\operatorname{Im} \bar{\partial}}$.

A central result is the Čhech-Dolbault isomorphism, which follows from the Čhech-deRham isomorphism see [72] page 43-44 and the $\bar{\partial}$-Poincaré Lemma. It states for sheaves of vectors fields $F$ that

$$
H^{q}\left(M, \Omega^{p}(F)\right) \cong H_{\bar{\partial}}^{p, q}(M, F)
$$

For example $H^{q}\left(M, \wedge^{p} T^{*} M\right) \cong H^{p, q}(M, T M)=: H^{p, q}(M)$.

\footnotetext{
${ }^{32}$ On an almost complex manifold one can project $r$-forms $\Omega$ with $p$ P's and $q \bar{P}$ 's $(r=p+q)$ to $(p, q)$-forms $\Omega^{p, q}$. As $J$ depends on the coordinates one gets $\mathrm{d} \Omega^{p, q}=(\mathrm{d} \Omega)^{p-1, q+2}+(\mathrm{d} \Omega)^{p, q+1}+(\mathrm{d} \Omega)^{p+1, q}+(\mathrm{d} \Omega)^{p+2, q-1}$ and one may define $\partial \Omega^{p, q}=(\mathrm{d} \omega)^{p+1, q}$ and $\bar{\partial} \Omega^{p, q}=(\mathrm{d} \Omega)^{p, q+1}$. One can check that the condition $\bar{\partial}^{2}=0$ is equivalent to $N_{c d}^{b} \equiv 0$.
} 


\subsection{Kähler manifolds}

A hermitian metric is a positive-definite inner product $T M \otimes \bar{T} M \rightarrow \mathbf{C}$. Locally it can be given by a covariant tensor $\sum_{i, j}^{n} g_{i \bar{j}}(w) \mathrm{d} x^{i} \otimes \mathrm{d} x^{\bar{j}}$ such that $\overline{g_{i j}}=g_{j \bar{\imath}}$ and $\forall v^{i} \in \mathbf{C}$ one has $v^{i} g_{i j} v^{\bar{j}}>0$, if not all $v^{i}=0$. Note that the first index of $g_{i \bar{j}}$ is only summed over the unbarred $i=1, \ldots, n$ and the second only over barred $\bar{j}=\overline{1}, \ldots, \bar{n}$ indices respectively. To define a hermitian metric an almost complex structure is sufficient. Hermiticity is the condition $g(X, Y)=g(J X, J Y)$ on the real metric, which becomes

$$
g_{m n}=J_{m}^{a} J_{n}^{b} g_{a b}
$$

in coordinates. It does not constrain $M$ further than admitting $J$ and any metric say $g^{\prime}$, because for any such $g^{\prime}$ the metric $g_{m n}=\frac{1}{2}\left(g_{m n}^{\prime}+J_{m}^{a} J_{n}^{b} g_{a b}^{\prime}\right)$ is hermitian. In particular on any complex manifold we can define a hermitian metric see [98] Chap 3.5. Multiplying (7.11) with $J_{p}^{m}$, defining $J_{n m}=J_{n}^{a} g_{a m}$ and using $J_{p}^{m} J_{m}^{a}=-\delta_{p}^{a}$ we see that $J_{n m}=-J_{m n}$. Hence we can define a 2-form $\omega=J_{n m} \mathrm{~d} w^{n} \wedge \mathrm{d} w^{m}$. In complex notation this becomes

$$
\omega=i \sum_{i, j=1}^{n} g_{i j} \mathrm{~d} x^{i} \wedge \mathrm{d} x^{\bar{j}}
$$

This is a real form $\bar{\omega}=\omega$ of type $(1,1)$ and is called the fundamental form associated to the hermitian metric. Because ${ }^{33} g:=\operatorname{det}\left(g_{i \bar{j}}\right)>0$ one gets by wedging $\omega$ n-times

$$
\mathrm{vol}=\frac{\omega^{n}}{n !}=i^{n} \operatorname{det}\left(g_{i \bar{j}}\right) \mathrm{d} x^{1} \wedge \mathrm{d} \bar{x}^{1} \wedge \ldots \wedge \mathrm{d} x^{n} \wedge \mathrm{d} \bar{x}^{n}=2^{n} \operatorname{det}\left(g_{i j}\right)^{\frac{1}{2}} \mathrm{~d} w^{1} \wedge \ldots \wedge \mathrm{d} w^{2 n}
$$

a positive volume form on $M$, which implies also that $M$ is orientable.

A hermitian metric whose fundamental form is closed $\mathrm{d} \omega=0$ is called a Kähler metric. A complex manifold endowed with a Kähler metric is called a Kähler manifold. d $\omega=0$ implies $\partial \omega=\bar{\partial} \omega=0$, which is equivalent to $\partial_{k} g_{i \bar{j}}=\partial_{i} g_{k \bar{j}}$ and $\bar{\partial}_{\bar{k}} g_{i \bar{j}}=\bar{\partial}_{\bar{j}} g_{i \bar{k}}$. The latter equations are integrability conditions for the existence of a local Kähler potential $K(x, \bar{x})$ which is real and yields the metric as follows

$$
g_{i \bar{j}}=\partial_{i} \partial_{\bar{j}} K(x, \bar{x})=-\frac{1}{2} \mathrm{~d}(\partial-\bar{\partial}) K(x, \bar{x}) .
$$

Note that despite the form above $\omega$ cannot be exact. For if $\omega=\mathrm{d} A$ would have been exact 7.13 could not be true, because using Stokes theorem the integral $\int \omega^{n}$ would be zero. That means that $(\partial-\bar{\partial}) K$ is not globally defined. Indeed as far as the definition of $\omega$ goes $K(x, \bar{x})$ only needs to be defined up to a Kähler transformation $K(x, \bar{x}) \rightarrow K(x, \bar{x})+f(x)+\bar{f}(\bar{x})$, so $e^{K}$ will be a section of a nontrivial line bundle over $M$. In general two Kähler forms $\omega$ and $\omega^{\prime}$ are in the same class in $H^{2}(M, \mathbf{R})$, if we can find a smooth global real function $\phi$ on $M$ and

$$
\omega^{\prime}=\omega+\partial \bar{\partial} \phi(x, \bar{x})
$$

\footnotetext{
${ }^{33}$ Note in coordinates $x^{i}, x^{\bar{i}}$ one has the block form $g_{n m}=\left(\begin{array}{cc}0 & g_{\mu \bar{v}} \\ g_{\sigma \bar{\rho}} & 0\end{array}\right)$ and e.g. [21] defines $g:=\operatorname{det}\left(g_{n m}\right)=$ $\operatorname{det}^{2} g_{\mu \bar{v}}$
} 
Above property (7.14) simplifies the expressions for the Christoffel symbols and the curvature tensors

$$
\begin{aligned}
& \text { a.) } \Gamma_{i j}^{k}=g^{k \bar{l}} \partial_{i} g_{j \bar{l}}, \quad \Gamma_{\bar{i} \bar{j}}^{\bar{k}}=g^{l \bar{k}} \bar{\partial}_{\bar{i}} g_{l \bar{j}} \\
& \text { b.) } R_{i \bar{j} k \bar{l}}=-\partial_{i} \bar{\partial}_{\bar{j}} g_{k \bar{l}}+g^{m \bar{n}}\left(\partial_{i} g_{k \bar{n}}\right)\left(\bar{\partial}_{\left.\bar{j} g_{m \bar{l}}\right),} R_{i \bar{j} k}{ }^{l}=-\bar{\partial}_{\bar{j}} \Gamma_{i k}^{l}\right. \\
& \text { c.) } R_{i \bar{j}} \equiv g^{k \bar{l}} R_{i \bar{j} k \bar{l}}=-\partial_{i} \bar{\partial}_{\bar{j}} \log \operatorname{det}\left(g_{i \bar{j}}\right) .
\end{aligned}
$$

Note that the pure index Christoffel symbols are the only non-vanishing ones and that $R_{i j \bar{k} l}=$ $R_{k \bar{j} \bar{l} \bar{l}}=R_{i \bar{l} \bar{j} \bar{j}}$, because of the integrability condition. The other non vanishing components of the Ricci tensor are of type $R_{\bar{j} i k \bar{l}}, R_{i \bar{j} \bar{l} \bar{l}}$ and $R_{\bar{j} i \bar{l} k}$. From the Ricci tensor one defines the Ricci form

$$
\mathscr{R}=i R_{i \bar{j}} \mathrm{~d} x^{j} \wedge \mathrm{d} x^{\bar{j}}=-i \partial \bar{\partial} \log \operatorname{det}\left(g_{i \bar{j}}\right)=\frac{i}{2} \mathrm{~d}(\partial-\bar{\partial}) \log \operatorname{det}\left(g_{i \bar{j}}\right) .
$$

It satisfies $\mathrm{d} \mathscr{R}=0$, but is not exact, despite the form it is written above, because $\log \operatorname{det}\left(g_{i \bar{j}}\right)$ is a density and not a function.

We now turn to harmonic theory for complex manifolds. On $(p, q)$-forms $\phi=\frac{1}{p ! q !} \phi_{i_{1}, \ldots, i_{p}, \bar{j}_{1} \ldots, j_{q}} \mathrm{~d} x^{i_{1}} \wedge$ $\ldots \wedge \mathrm{d} x^{i_{p}} \wedge \mathrm{d} x^{\bar{j}_{1}} \wedge \ldots \wedge \mathrm{d} x^{\bar{j}_{q}}$ we have a local inner product defined by a hermitian metric

$$
(\phi, \psi)(x)=\frac{1}{p ! q !} \phi_{i_{1} \ldots i_{p} \bar{j}_{1} \ldots \bar{j}_{q}} \psi^{i_{1} \ldots i_{p} \bar{j}_{1} \ldots \bar{j}_{q}}
$$

where $\psi^{i_{1} \ldots i_{p} \bar{j}_{1} \ldots \bar{j}_{q}}=g^{i_{1} \bar{l}_{1}} \ldots g^{i_{p} \bar{l}_{p}} g^{k_{1} \bar{j}_{1}} \ldots g^{k_{q} \bar{j}_{q}} \overline{\psi_{k_{1} \ldots k_{q} \bar{l}_{1} \ldots \bar{l}_{p}}}$. With this we can define an global inner product $A^{p, q} \times A^{p, q} \rightarrow \mathbf{C}$

$$
(\phi, \psi)=\int_{M}(\phi, \psi)(x) \mathrm{vol}
$$

with

$$
(\phi, \psi)=\overline{(\psi, \phi)}, \quad(\phi, \phi)>0 \text { unless } \phi=0,
$$

which makes $A^{p, q}$ in a pre-Hilbert space. One can define the Hodge operator ${ }^{34} *: A^{p, q} \rightarrow A^{n-q, n-p}$ i.e. $*: \psi \mapsto * \psi$ by

$$
(\phi, \psi) \operatorname{Vol}=\phi \wedge * \bar{\psi},
$$

with $\bar{\psi}=\frac{1}{p ! q !} \overline{\psi_{i_{1} \ldots i_{p}, \bar{j}_{1} \ldots \bar{j}_{q}} \mathrm{~d} x^{i_{1}} \wedge \ldots \wedge \mathrm{d}^{i_{p}} \wedge \mathrm{d} x^{\bar{j}_{1}} \wedge \ldots \wedge \mathrm{d} x^{\bar{j}_{q}}}=\frac{1}{p ! q !} \bar{\psi}_{j_{1} \ldots j_{q}, \bar{l}_{1} \ldots \bar{l}_{p}} \mathrm{~d} x^{j_{1}} \wedge \ldots \wedge \mathrm{d}^{j_{q}} \wedge \mathrm{d} x^{\bar{l}_{1}} \wedge$ $\ldots \wedge \mathrm{d} x^{\bar{t}_{p}}$ and $\overline{\psi_{i_{1} \ldots i_{p}, \bar{j}_{1} \ldots \bar{j}_{q}}}=(-1)^{p q} \bar{\psi}_{j_{1} \ldots j_{q}, \bar{l}_{1} \ldots \bar{l}_{p}}$. Explicitly

$$
* \psi=\frac{i^{n}(-1)^{n(n-1) / 2+n p}}{p ! q !(n-p) !(n-q) !} g \varepsilon_{\bar{j}_{1} \ldots \bar{j}_{n-p}}^{k_{1} \ldots k_{p}} \varepsilon_{i_{1} \ldots \bar{i}_{n-q}}^{\bar{l}_{1} \ldots \bar{l}_{q}} \psi_{k_{1} \ldots k_{p}, \bar{l}_{1} \ldots \bar{l}_{q}} \mathrm{~d} x^{i_{1}} \wedge \ldots \wedge \mathrm{d}^{i_{n-q}} \wedge \mathrm{d} x^{\bar{j}_{1}} \wedge \ldots \wedge \mathrm{d} x^{\bar{j}_{n-p}}
$$

One checks $* \bar{\psi}=\overline{* \psi}$ and $* * \psi=(-1)^{p q} \psi$ for $\psi$ a $(p, q)$-form.

With the norm $(\cdot, \cdot)$ we can define the adjoint operators $\partial^{*}: A^{p, q} \rightarrow A^{p-1, q}$ and $\bar{\partial}^{*}: A^{p, q} \rightarrow$ $A^{p, q-1}$ by

$$
\left(\partial^{*} \psi, \phi\right):=(\psi, \partial \phi), \quad \text { and } \quad\left(\bar{\partial}^{*} \psi, \phi\right):=(\psi, \bar{\partial} \phi)
$$

respectively. On a compact manifold one has $\bar{\partial}^{*}=-* \partial *$. With the adjoint operator one can define beside the de Rham Laplacian $\Delta_{\mathrm{d}}=\mathrm{dd}^{*}+\mathrm{d}^{*} \mathrm{~d}$ the Laplacians $\Delta_{\partial}=\partial \partial^{*}+\partial^{*} \partial$ and $\Delta_{\bar{\partial}}=\bar{\partial} \bar{\partial}^{*}+\bar{\partial}^{*} \bar{\partial}$. The Hodge theorem states that every element $\phi \in A^{p, q}$ has an unique orthogonal decomposition into

\footnotetext{
${ }^{34}$ Here the conventions are as in [98]. The $*$ operator in [2] maps $*_{g h}: A^{p, q} \rightarrow A^{n-p, n-q}$, so it involves an additional complex conjugation $*_{g h} \psi=*_{k o} \bar{\psi}$.
} 
a harmonic form $h$, an exact piece $\bar{\partial} \xi$ with $\xi \in A^{p, q-1}$ and a co-exact piece $\bar{\partial}^{*} \eta$ with $\eta \in A^{p, q+1}$ i.e.

$$
A^{p, q}=\mathscr{H}^{p, q} \oplus \bar{\partial} A^{p, q-1} \oplus \bar{\partial}^{*} A^{p, q+1} .
$$

This is in analogy with the de Rham decomposition $A^{p}=\mathscr{H}^{p} \oplus \mathrm{d} A^{p-1} \oplus \mathrm{d}^{*} A^{p+1}$. The usual argument shows that if $\phi$ is closed, i.e. $\bar{\partial} \phi=0$, then the $\bar{\partial}^{*} \eta$ piece in the decomposition is zero, because $\bar{\partial} \phi=\bar{\partial} \bar{\partial}^{*} \eta$ and thus $0=(\bar{\partial} \phi, \eta)=\left(\bar{\partial}^{*} \eta, \bar{\partial}^{*} \eta\right)$, which implies $\bar{\partial}^{*} \eta=0$. This in turn means that every $\bar{\partial}$ closed form can be uniquely decomposed into a harmonic form w.r.t. $\Delta_{\bar{\partial}}$ and a $\bar{\partial}$ exact piece, which implies $H_{\bar{\partial}}^{p, q}(M) \cong \mathscr{H}^{p, q}(M)$.

Using $\left(\bar{\partial}^{*} \psi\right)_{i_{1} \ldots i_{p} \bar{j}_{2} \ldots \bar{j}_{p}}=(-1)^{p+1} \nabla^{\bar{j}_{1}} \psi_{i_{1} \ldots i_{p} \bar{j}_{1} \bar{j}_{2} \ldots \bar{j}_{q}}$ one can show that the Kähler $\omega$ form is harmonic. Hence $h^{1,1}(M) \geq 1$ on a Kähler manifold. Similarly one shows that all $\omega^{m}, m=1, \ldots, n$ are nontrivial elements in $H^{m, m}(M)$. A very important result for Kähler manifolds is the Laplacians are all equivalent

$$
\Delta_{\partial}=\Delta_{\bar{\jmath}}=\frac{1}{2} \Delta_{d},
$$

where $\Delta_{\bar{\partial}}=\bar{\partial} \bar{\partial}^{*}+\bar{\partial}^{*} \bar{\partial}, \Delta_{\partial}=\partial \partial^{*}+\partial^{*} \partial$ and $\Delta_{\mathrm{d}}=\mathrm{dd}^{*}+\mathrm{d}^{*} \mathrm{~d}$. As a consequence of (7.25) $\Delta_{\mathrm{d}}$ like $\Delta_{\partial}$ and $\Delta_{\bar{\partial}}$ does not change the $(p, q)$-type and taking the harmonic forms as unique representatives we have a decomposition of the deRham cohomology groups

$$
H^{r}(M)=\bigoplus_{p+q=r} H^{p, q}(M)
$$

Let us note for further reference that the action of $\Delta_{\mathrm{d}}$ on $p$-forms $\omega$ can be expressed in terms of covariant derivatives and the curvature tensors as

$$
\left(\Delta_{\mathrm{d}} \omega\right)_{\mu_{1} \ldots \mu_{p}}=-\nabla^{v} \nabla_{v} \omega_{\mu_{1} \ldots \mu_{p}}-p R_{v\left[\mu_{1}\right.} \omega_{\left.\mu_{2} \ldots \mu_{p}\right]}^{v}-\frac{1}{2} p(p-1) R_{v \rho\left[\mu_{1} \mu_{2}\right.} \omega_{\left.\mu_{3} \ldots \mu_{p}\right]}^{v \rho}
$$

By consideration of type follows that every holomorphic $(p, 0)$-form $\omega$ is harmonic and vice versa. We have $\bar{\partial}^{*} \omega=0$ as it maps to $A^{p,-1}$ which is trivial. If $\Delta_{\bar{\partial}} \omega=0$ then from $\bar{\partial} * \bar{\partial} \omega=0$ follows $\bar{\partial} \omega=0$.

Forms of Kähler manifolds are related by complex conjugation $\overline{A^{p, q}}=A^{q, p}$, which implies for the cohomology groups $H^{p, q}(M)=H^{q, p}(M)$, since complex conjugation commutes with $\Delta_{\mathrm{d}}$. The star operator $*: A^{p, q} \rightarrow A^{n-q, n-p}$ is another bijection which commutes with $\Delta_{\mathrm{d}}$ and hence

$$
H^{q, p}(M)=H^{p, q}(M)=H^{n-q, n-p}(M) .
$$

Let us mention briefly further important facts about Kähler manifolds. The property of the Christoffel symbol to have only pure indices leads to the fact that parallel transport of a vector generates only the holonomy group $U(n) \in S O(2 n)$ rather then $S O(2 n)$, which would be the holonomy of a generic orientable manifold.

Another well known fact is that $\mathbf{P}^{n}$ is a Kähler manifold. This can be established by giving with the Fubini-Study metric an explicit. In the $\mathscr{U}_{i}, i=0, \ldots, n$ patches the Kähler potential is given by $K^{(i)}\left(x^{(i)}, \bar{x}^{(i)}\right)=\log \left(1+\left|x^{(i)}\right|^{2}\right)$, where $\left|x^{(i)}\right|^{2}=\sum_{j \neq i}\left|x_{j}^{(i)}\right|^{2}$. Using (7.3) we see that $K^{(i)}\left(x^{(i)}, \bar{x}^{(i)}\right)=K^{(j)}\left(x^{(j)}, \bar{x}^{(j)}\right)-\log \frac{x_{i}}{x_{j}}-\log \frac{\bar{x}_{i}}{\bar{x}_{j}}$. The latter two terms are holomorphic and antiholomorphic functions respectively on $\mathscr{U}_{i} \cap \mathscr{U}_{j}$. Hence they do not affect the metric $g_{i \bar{j}}=\partial_{i} \partial_{\bar{j}} K(x, \bar{x})$, 
which is globally well defined. Dropping the index for the patch we get

$$
\omega=i \partial \bar{\partial} \log \left(1+|x|^{2}\right)=i\left(\frac{\mathrm{d} x^{i} \wedge \mathrm{d} x^{\bar{i}}}{1+|x|^{2}}-\frac{\bar{x}^{i} \mathrm{~d} x^{i} \wedge x^{j} \mathrm{~d} x^{\bar{j}}}{\left(1+|x|^{2}\right)^{2}}\right) .
$$

This defines a positive-definite metric. With $\operatorname{det}\left(g_{i, \bar{j}}\right)=\frac{1}{\left(1+|x|^{2}\right)^{n+1}}$ one calculates the Ricci tensor $R_{i \bar{j}}=-\partial_{i} \partial_{\bar{j}} \log \operatorname{det}\left(g_{i \bar{j}}\right)=(n+1) g_{i \bar{j}}$. If the Ricci tensor is proportional to the Kähler metric one calls the metric Kähler-Einstein.

\subsection{Characteristic classes of holomorphic vector bundles}

In the last section we encountered the holomorphic tangent bundle of $M$ as an example of a holomorphic vector bundle $E$ with a hermitian metric, which we call $h_{a b}$ in the general case. The connection one form

$$
A_{k}=\left(\partial_{k} h\right) h^{-1}, \quad A_{\bar{k}}=0
$$

defines the unique affine connection, which is compatible with the hermitian metric, i.e $\nabla h=0$, and compatible with the complex structure. One defines the curvature two form as $F=\mathrm{d} A+A \wedge A$. The differential geometry approach to Chern classes $c_{i}(E) \in H^{2 i}(M, \mathbf{R})$ of a rank $r$ holomorphic vector bundle is to define them in terms symmetric function of the eigenvalues of the curvature form as

$$
c(E)=\operatorname{det}\left(1+\frac{i}{2 \pi} F\right)=1+\sum_{i} c_{i}(E)=1+\frac{i}{2 \pi} \operatorname{Tr} F+\ldots
$$

and to prove then that they do not depend on the metric[12][140].

Topologically one can represent the Chern class $c_{k}$ as the Poincaré dual to the degeneracy cycle

$$
D_{r-k+1}(\sigma)=\left\{x: \sigma_{1}(x) \wedge \ldots \sigma_{r-k+1}(x)=0\right\},
$$

where $r-k+1$ generic $\mathscr{C}^{\infty}$ sections $\sigma_{i}$ of $E$ become linearly dependent. This is described as Gauss Bonnet formula II in Chap 3.3 of [72], see also [58] [31] for the approach using classifying spaces. The simplest example of the above dual descriptions arise for line bundles $\mathscr{L}$. Let $|\sigma|^{2}$ be a metric on a line bundle $L$, where $\sigma$ is a section of $L$. Local trivialization of $L$ are $\phi:\left.L\right|_{U} \rightarrow U \times \mathbf{C}$, where $s_{U}$ is a holomorphic function and $|\sigma|^{2}=h(x)\left|s_{U}\right|^{2}$ for some function $h(x)$, which is positive if the metric is. The curvature 2 -form given by

$$
\mathscr{R}=-\bar{\partial} \partial \log h(x)
$$

defines the Chern-class of $L$ represented by $c_{1}(L)=\frac{i}{2 \pi}[\mathscr{R}] \in H^{2}(M)$. This class is Poincare dual to the divisor class $[D]$ which defines $L$ and is uniquely recovered from $L$ as the locus where the generic section vanishes. As a corollary the first Chern class of a holomorphic vector bundle is also the first Chern class of the determinant bundle $L_{D}=\wedge^{r} E$

$$
c_{1}(E)=c_{1}\left(L_{D}\right)
$$

For the tangent bundle we identify the curvature 2-form $F$ with $\Theta_{\bar{i}}^{j}=g^{j \bar{p}} R_{i \bar{p} k \bar{l}} \mathrm{~d} x^{k} \wedge \mathrm{d} x^{\bar{l}}$ and get a representative for $c_{1}(T M)$ (which we also call $c_{1}(M)$ )

$$
c_{1}(M)=\frac{i}{2 \pi} \Theta_{i}^{i}=R_{k \bar{l}} \mathrm{~d} x^{k} \wedge \mathrm{d} x^{\bar{l}}=-\frac{i}{2 \pi} \partial \bar{\partial} \log \operatorname{det}\left(g_{k \bar{l}}\right) .
$$


The canonical line bundle is the determinant line bundle of the holomorphic tangent bundle $K_{M}=\wedge^{n} T^{* 1,0} M$. By (7.34) and (7.35) we have therefore

$$
-2 \pi c_{1}\left(K_{M}\right):=-2 \pi c_{1}\left(\wedge^{n} T^{* 1,0} M\right)=-2 \pi c_{1}\left(T^{*} M\right)=2 \pi c_{1}(T M) .
$$

Let us derive this also using as an explicit representative of the Chern class the curvature 2-form. Given a complex structure and a Kähler metric $g_{i \bar{j}}$ we have a connection on $T^{* 1,0} M$ described by the holomorphic Christoffel symbols. This connection induces a connection on the line bundle $K_{M}$ and a straightforward calculation shows on total antisymmetric forms $\left[\nabla_{i}, \nabla_{\bar{j}}\right] \omega_{i_{1} \ldots, i_{n}}=-R_{i \bar{j}} \omega_{i_{1} \ldots, i_{n}}$ Therefore we can identify $h(x)$ of (7.33) with $\operatorname{det}^{-1}\left(g_{i j}\right)$ and by (7.33) the first Chern class of $K_{M}$ is

$$
-2 \pi c_{1}\left(K_{M}\right)=[\mathscr{R}]=2 \pi c_{1}(T M) .
$$

If one uses the Poincaré Hopf theorem that the Euler number $\chi(M)$ of a manifold of $\operatorname{dim} n$ is given by the sum of indices of zeros of a generic vector field, i.e. a section of the tangent bundle, then by (7.32) the dual to $c_{n}(T M)$ is $D_{1}$. Counting these zeros leads then to the Gauss-Bonnet formula

$$
\chi(M)=D_{1} \cap M=\int_{M} c_{n}(T M) .
$$

Let us discuss further properties of the Chern classes. By (7.31) one has $c_{0}(E)=1, c_{k>r}(E)=$ 0 and the Whitney product formula $c(E \oplus F)=c(E) C(F)$ from the properties of the determinant, see [16] for a proof from the topological definition. It is also easy to see[72] that

$$
c_{k}\left(E^{*}\right)=(-1)^{k} c_{k}(E)
$$

and $c_{k}(f(E))=f^{*} c_{k}(E)$ for $f: M \rightarrow M^{\prime}$ a differentiable mapping. A further important property is the splitting principle [16]. For an exact sequence of holomorphic vector bundles or sheaves $0 \rightarrow E \rightarrow F \rightarrow G \rightarrow 0$ one has $c(F)=c(E) c(G)$. One considers often classes $x_{i}$ such that $c(E)=$ $\prod_{i=1}^{r}\left(1+x_{i}\right)$ where $x_{i}$ are Chern classes of line bundles. One reason that this is useful is that the splitting principle implies that if one wants to derive polynomial identities among Chern classes of vector bundles, one may replace the vector bundles by direct sums of line bundles. This opens up a calculational machinery with classes, which behave e.g. more natural on direct products as the Chern character $\mathrm{Ch}(E)=\sum_{i=1}^{r} e^{x_{i}}$. All expressions are polynomial, defined by expanding up to degree $r$ in $x_{i}$. Obviously $\operatorname{Ch}(E \otimes F)=\mathrm{Ch}(E)+\operatorname{Ch}(F)$ and $\operatorname{Ch}(E \otimes F)=\operatorname{Ch}(E) \operatorname{Ch}(F)$. A little playing with symmetric functions reveals $\mathrm{Ch}(E)=r+c_{1}+\frac{1}{2}\left(c_{1}^{2}-2 c_{2}\right)+\frac{1}{6}\left(c_{1}^{3}-3 c_{1} c_{2}+3 c_{3}\right)+\ldots$, where we set $c_{k}=c_{k}(E)$. Similar is the Todd genus defined $\operatorname{td}(E)=\prod_{i=1}^{r} \frac{e^{x_{i}}}{1-e^{x_{i}}}=1+\frac{1}{2} c_{1}+\frac{1}{12}\left(c_{1}^{2}+\right.$ $\left.c_{2}\right)+\frac{1}{24} c_{1} c_{2}+\ldots$. A central theorem is the Hirzebruch-Riemann-Roch formula, which gives the arithmetic genus $\chi(E)=\sum_{k}(-1)^{k} h^{k}(E)$ of a vector bundle over a manifold $M$ [81]

$$
\chi(E)=\int_{M} \operatorname{ch}(E) \wedge \operatorname{td}(T X) .
$$

In sections 5.1.5.2 and 6.11 we needed applications of (7.40). Namely to count the deformation space (4.15) of a Riemann surface ${ }^{35} \Sigma_{g}$. As seen in section 6.2 the complex structure moduli of the

\footnotetext{
${ }^{35}$ This related by the Atiyah-Singer index formula to the index of the Dirac operator and hence to the ghost zero modes. An overview about index formulas for physicist can be found in [48] and the connections to the zero modes is in explained e.g. in [126].
} 
metric are given by elements in the Čheck cohomology group $H^{1}(T)$ with $T=T \Sigma$ and for $g>1$ there are no conformal Killing vectors generating global diffeomorphims i.e. one has $h^{0}(T)=0$. However for $g=1$ the shift $z \rightarrow z+\lambda$ on the torus accounts for $h^{0}=1$ and for $g=0$ the three generators of $\operatorname{PSL}(2, \mathbf{C}) z \rightarrow \frac{a z+c}{c z+d}$ on $S^{2}$ account for $h^{0}=3$. For a vector bundle $V$ of rank $r$ over the Riemann surface $\Sigma$ the formula (7.40) gives

$$
h^{0}(\Sigma, V)-h^{1}(\Sigma, V)=\int_{\Sigma} \operatorname{ch}(V) \wedge \operatorname{td}(T)=\int_{\Sigma}\left(r+c_{1}(V)\right)\left(1+\frac{1}{2} c_{1}(T)=\int_{\Sigma} c_{1}(V)+r(1-g) .\right.
$$

The virtual dimension of the deformation space is obtained by setting $V=T$ with rank 1

$$
\operatorname{dim} \mathscr{M}_{g}=h^{1}(T)-h^{0}(T)=-\int_{\Sigma} \operatorname{ch}(T) \wedge \operatorname{td}(T)=3 g-3 .
$$

In the integral over the metric moduli space in string amplitudes one sacrifices in the $g=0,1$ cases $h^{0}=3,1$ additional parameters, the position of insertion points, to offset the negative contributions to (7.42) from the conformal Killing fields. Another application leads to the formula (5.10) describing the dimension of the deformation space of holomorphic maps $x: \Sigma \rightarrow M$. The movement of the curve in $M$ is described infinitesimally by a vector field $x^{i} \rightarrow x^{i}+\varepsilon \xi^{i}$ on $M$. The vector field must be holomorphic $\partial_{\bar{z}} \xi=0$ so that the deformed map stays holomorphic. Also we are not counting vector fields which correspond to reparametrizations of $\Sigma$. That is we look at elements of $H_{\bar{\partial}}^{0}\left(\Sigma, x^{*}(T M)\right)=H^{0}\left(x^{*}(T M)\right)$ and (7.40) gives us

$h^{0}\left(x^{*}(T M)\right)-h^{1}\left(x^{*}(T M)\right)=\int_{\Sigma}\left(\operatorname{dim}_{C} M+x^{*}\left(c_{1}(T M)\right)\right)\left(1+\frac{1}{2} c_{1}(T)\right)=c_{1}(T M) \cdot \beta+\operatorname{dim}_{C} M(1-g)$.

Generically the movement of the map is unobstructed and $H^{1}\left(x^{*}(T M)\right)=0$. In the case the above is also the dimension of the deformation space. In the case of Calabi-Yau three folds we get for genus 0 that the dimension of the deformation space is 3 . We can think about this in two ways. Either we don't fix points on $S^{2}$, then we have to mod out by the 3 dim automorphism group $P L(2, \mathbf{C})$ of $S^{2}$ and the expected dimension of the moduli space is 0 . That is the way the corrections in $\mathscr{F}(0)$ are interpreted. Or we kill $P L(2, \mathbf{C})$ by marking three points on the $S^{2}$ required to map into three divisors, which put three constraints and yields again a zero dimensional moduli space. That is the interpretation of corrections in $C_{i j k}(t)$.

\subsection{Metric Connection and Holonomy}

To describe spinor connection on curved spaces one introduces beside the curved indices $M, N, \ldots$ the flat tangent indices $A, B, \ldots$ which are lowered and raised with the flat metric $\eta^{A B}=$ $\operatorname{diag}(-1, \underbrace{1, \ldots, 1}_{D-1})$ and its inverse.

The Clifford algebra is defined by the anti commutator of $\left\{\Gamma^{A}, \Gamma^{B}\right\}=2 \eta^{A B}$. In the smallest representation the $\Gamma$ symbols are $2^{[D / 2]} \times 2^{[D / 2]}$ matrices. The generators of the Lorentz group in the spinor representation $\xi$ of dimension $2^{[D / 2]}$ are given by the commutator $T_{A B}^{s}=-\frac{i}{2} \Gamma_{A B}=$ $-\frac{i}{4}\left[\Gamma_{A}, \Gamma_{B}\right]$, i.e. $\xi \mapsto \exp \left(i \omega^{A B} T_{A B}^{s}\right) \xi$ under the spin group which is a cover of proper, ortochronous Lorentzgroup $S O_{\uparrow}^{+}(1, D-1)$. We do not display spinor indices $a, b \ldots$ like in $\tilde{\xi}_{a}=\left(\Gamma^{A}\right)_{a}^{b} \xi_{b}, a, b=$ $1, \ldots,[D / 2]$ explicitly. For more on spin representations in various dimensions, see e.g. [126]. 
The relation to curved indices $M, N \ldots$, lowered and raised by the curved metric $G_{M N}$ and its inverse $G^{M N}$, is provided by the $D$-bein $e_{M}^{A}$ and its inverse $e_{B}^{N}\left(e_{M}^{A} e_{A}^{N}=\delta_{M}^{N}\right.$ and $\left.e_{M}^{A} e_{B}^{M}=\delta_{B}^{A}\right)$ which fulfills $G_{M N}=e_{M}^{A} e_{N}^{B} \eta_{A B}$. One has $\Gamma^{A}=e_{M}^{A} \Gamma^{M}$ and $\Gamma^{M}=e_{A}^{M} \Gamma^{A}$ etc., from which follows $\left\{\Gamma^{M}, \Gamma^{N}\right\}=2 G^{M N}$. A torsion free $\Gamma_{M N}^{P}=\Gamma_{N M}^{P}$ Riemann connection leaves the metric invariant

$$
\nabla_{S} G_{M N}=0=\partial_{S} G_{M N}-\Gamma_{S M}^{P} G_{P N}-\Gamma_{S N}^{L} G_{P M}
$$

which implies the formula for the Christoffel Symbols

$$
\Gamma_{M N}^{S}=\frac{1}{2} G^{S P}\left(\partial_{M} G_{P N}+\partial_{N} G_{M P}-\partial_{P} G_{M N}\right) .
$$

The spin connection $\omega_{M B}^{A}$ is defined as

$$
\nabla_{M} e_{N}^{A}=\partial_{M} e_{N}^{A}-\Gamma_{M N}^{P} e_{P}^{A}+\omega_{N B}^{A} e_{M}^{B}
$$

which implies that

$$
\omega_{M}^{A B}=\frac{1}{2}\left(\Omega_{M N R}-\Omega_{N R M}+\Omega_{R M N}\right) e^{N A} e^{R B}, \quad \text { with } \quad \Omega_{M N R}=\left(\partial_{M} e_{N}^{A}-\partial_{N} e_{M}^{A}\right) e_{A R}
$$

The connection on a spinor is then

$$
\partial_{M} \xi=\left(\partial_{M}+\frac{i}{2} \omega_{M}^{A B} T_{A B}^{S}\right) \xi
$$

and for any other representation carrying only flat indices of the tangent space one has to replace $T_{A B}^{S}$ by the appropriate generator of the Lorentz group, i.e. $T_{A B}^{v}=\eta_{A C} \delta_{B}^{D}-\eta_{B C} \delta_{A}^{D}$ for vectors etc.

If a vector $V^{N}$ is parallel transported around a infinitesimal rectangle along two tangent vectors $\frac{\partial}{\partial X_{A}}$ and $\frac{\partial}{\partial X_{B}}$ with area element $\sigma^{A B}=-\sigma^{B A}$ its infinitesimal rotation is $\delta V^{L}=-\frac{1}{2} \delta \sigma^{M N} R_{M N}^{L}{ }_{P} V^{P}$, which is one way to explain the effect of curvature

$$
\left[\nabla_{M}, \nabla_{N}\right] V_{P}=-R_{M N P}^{S} V_{S} \text {, with } R_{M N P}^{S}=\partial_{M} \Gamma_{N P}^{S}-\partial_{N} \Gamma_{M P}^{A}+\Gamma_{N P}^{B} \Gamma_{M B}^{S}-\Gamma_{M P}^{B} \Gamma_{N B}^{S} .
$$

Note $R_{N O P}^{M}=-R_{N P O}^{M}$ and also for a Kähler manifold the only non vanishing elements of $R_{i \bar{j} l}^{k}$ is pure in $k, l$. That means that a holomorphic vector stays holomorphic under parallel transport and $\delta \sigma^{m n} R_{m n ~ l}^{k}$ spans the Lie algebra of $U(n)$. Near the identity $U(n) \cong S U(n) \times U(1)$ and the $U(1)$ part is generated by the trace part of the Riemann tensor which is the Ricci tensor $\delta \sigma^{m n} R_{m n k}^{k}=$ $-4 \delta \sigma^{\mu \bar{v}} R_{\mu \bar{v}}$.

Once one knows the holonomy group Hol on vectors the transformation properties of tensors, forms and spinors becomes a matter of representation theory. In particular the following holds see e.g. [86. If $\mathrm{Hol}$ is the holonomy group of a connection $\nabla$ on $T M$ on a simply connected manifold $M$ then a tensor section $S \in \bigotimes^{i} T M \otimes \bigotimes^{j} T^{*} M$ is covariantly constant (parallel) iff $\left.S\right|_{x_{0}}$ is locally fixed by Hol.

The restriction to simply connected is quite important. Non simply connected manifolds can have monodromy even if they are flat. Consider e.g. the easy example of a non-simply connected space which is topologically $M=S^{1} \times \mathbf{R}^{2}$ with the metric

$$
\mathrm{d}^{2} s=R^{2} \mathrm{~d}^{2} \theta+\left(\mathrm{d} x^{i}+T_{j}^{i} x^{j} \mathrm{~d} \theta\right)^{2},
$$

where $T=\left(\begin{array}{rr}0 & 1 \\ -1 & 0\end{array}\right)$ is the generator of $S O(2)$ rotations in $\mathbf{R}^{2} . M$ is flat, yet a vector parallel transported around the $S^{1}$ gets rotated in the $\mathbf{R}^{2}$ directions. Similar examples a flat connection on tori, with monodromy. In the case of a gauge connection we call such configurations Wilson lines. 


\subsection{Calabi-Yau manifolds}

A general Calabi-Yau manifold is a compact Kähler manifold $M$ with vanishing first Chern class $c_{1}(T M)=0$. The following statements are essentially equivalent for complex $n$ dimensional Kähler manifolds $M$, up to some important subtleties for non-simply connected cases, which we discuss below. Together with the Kähler property they are used to define a (general) Calabi-Yau manifold

- a) The canonical class is trivial.

- b) The first Chern class of the tangent bundle vanishes ${ }^{36} c_{1}(T M)=0$.

- c) It exists a Kähler metric $g$ whose Ricci tensor vanishes $R_{i \bar{j}}(g)=0$.

- d) There exists an up to a constant unique nowhere vanishing holomorphic $(n, 0)$ form $\Omega$.

- e) The holonomy group Hol of $M$ is a subgroup of $S U(n)$.

- f) $M$ admits a pair of globally defined covariantly constant (parallel) spinors $\xi$ and $\bar{\xi}$ of opposite chirality if $n$ is odd and of the same chirality if $n$ is even.

Complex tori of all dimensions are general Calabi-Yau with trivial holonomy. In $\operatorname{dim}_{\mathbf{C}}=1$ the torus is the only topological type of Calabi-Yau manifold. In $\operatorname{dim}_{\mathbf{C}}=2$ the $K 3$-surface is the only topological Calabi-Yau manifold with $G=S U(2)$, while in $\operatorname{dim}_{\mathrm{C}}=3$ the number of different topological types of Calabi-Yau manifolds is $>10^{5}$. This estimate comes from explicit construction mostly of hypersurface and complete intersections in toric ambient spaces, see also Sec. 7.7 .

The question one is mainly interested in for physical applications, is how many super symmetries are unbroken in compactifications to four dimensions. An important situation is when the number of supercharges is reduced by $1 / 4$ by a compactification of the ten dimensional supergravity on the six real dimensional internal manifold $M$. This is the case if $\xi$ and $\bar{\xi}$ are the only covariantly constant spinors [24]. This in turn holds generically, without further non-trivial background fields, if the holonomy is the full $S U(3)$, i.e $\mathrm{Hol}=S U(3)$ and in an interesting special case namley the $T_{\mathbf{C}}^{1} \times K 3 / Z_{2}$ FHSV model, which has $\mathrm{Hol}=S U(2) \times Z_{2}$. Important applications emerging form this scheme are the $10 \mathrm{~d}$ heterotic compactification, which leads to $N=1$ supersymmetry in $4 \mathrm{~d}$ and the $10 \mathrm{~d}$ type II compactifications, which lead to $N=2$ supersymmetry in $4 \mathrm{~d}$. Other interesting examples for conceptual questions are compactification of type IIA or IIB to 6d on $K 3$, which has $\mathrm{Hol}=S U(2)$. This reduces the number of supercharges by $1 / 2$ and leads to $(1,1)$ and $(2,0)$ supersymmetry in $6 d$ respectively. A phenomenological very interesting compactifiction with $N=1$ in $4 \mathrm{~d}$ is $F$-theory compactification on an elliptically fibred Kähler manifold with $S U$ (4).

From the supergravity point of view the definition of a Calabi-Yau manifold, which covers the simplest physically interesting cases, is a compact Kähler manifold with $\mathrm{Hol}=S U(n)$. This implies that there are now exactly two covariant constant spinors. One excludes thereby cases involving non-simply connected manifolds such as $T_{\mathbf{C}}^{3}$ and $T_{\mathbf{C}}^{1} \times K 3$ and other products e.g. $K 3 \times K 3$. On nonsimply connected manifolds the relation between c.) and d.) is more subtle as they can have flat

\footnotetext{
${ }^{36} \mathrm{We}$ assume that we have a connection without torsion on $T M$.
} 
metrics, which do have non-trivial holonomy. They lead to interesting supersymmetry reduction by what is called generalized Scherk-Schwarz mechanism or geometrical Wilson lines [109]. However from the string point of view the important condition is the vanishing of the first Chern class $c_{1}(T M)=0$, which would have to be supplemented by the simply connectedness to restrict to get the $\frac{1}{4}$ supersymmetric case. The first reason is that this is the sufficient condition for the unbroken axial $U(1)$ on the world-sheet, necessary to define the B-twist. More importantly it is known that the non-linear $\sigma$-model is not conformally invariant for the Ricci-flat metric. The four loop $\beta$ function does not vanish in this geometry [74]. However it has be shown in [118][83] the possible counter terms that the total perturbative $\beta$-function can be set to zero by a change in the metric so that $\log \operatorname{det} g_{i \bar{j}}^{\text {string }}=\log \operatorname{det} g_{i \bar{j}}^{R \text { flat }}+\alpha(x, \bar{x})$, where $\alpha(x, \bar{x})$ is a globally defined real function on $M$, which is not $|f(x)|^{2}$ of a holomorphic one. By (7.17) this implies that the curvature two form becomes non-trivial by some non-vanishing exact terms, but the first Chern class stays of course trivial $c_{1}(T M)=0$. Ricci-flat flat manifolds are not a vacuum solutions of string theory. One may wonder whether the considerations about the covariantly constant spinors $\xi, \bar{\xi}$ make sense. They do, because what is required is that $\left(\nabla_{m}-\frac{i}{2} A_{m}\right) \xi=\left(\nabla_{m}+\frac{i}{2} A_{m}\right) \bar{\xi}$ is zero, where $A$ is a form potential for the Ricci-form $\mathscr{R}=\mathrm{d} A$, where $\bar{\partial}_{\bar{l}} \alpha=A_{\bar{l}}$ and $\partial_{i} \alpha=A_{i}$.

$\Omega \wedge \bar{\Omega}$ is proportional to the volume form and there is a natural normalization which makes $\operatorname{Re} \Omega$ a calibration

$$
\frac{\omega^{n}}{n !}=(-1)^{\frac{m(m-1)}{2}}\left(\frac{i}{2}\right)^{n} \Omega \wedge \bar{\Omega} .
$$

Imposing (7.51) reduces the freedom in the constant in e.) to a phase [86].

Let us now discuss the relation between the statements a.) to f.). In order to connect a.)-d.) to e.) and f.) we will assume that $M$ is simply connected and not of product form.

a.) $\leftrightarrow$ b.) follows from (7.36).

c.) $\rightarrow$ b.) is a simple consequence of the independence of the Chern classes on the choice of the Kähler metric. Once one knows that there exists a Ricci-flat metric clearly $c_{1}(T M)=0$ and that holds for all Kähler metrics.

b.) $\rightarrow$ c.) is a corollary to Yau' theorem [163], which proves the conjecture that E. Calabi formulated in (1956). It states that given the data

- (C.a) that for every given Kähler metric $g$, Kähler form $\omega$ and Ricci form $\mathscr{R}$ on $M$ and a real closed $(1,1)$ form $\mathscr{R}^{\prime}$, which represents the Chern class $[\mathscr{R}]=\left[\mathscr{R}^{\prime}\right]=2 \pi c_{1}(T M)$

one can construct

- (C.b) a unique metric $g^{\prime}$ on $M$ with associated Kähler form $\omega^{\prime}$ such that $\left[\omega^{\prime}\right]=[\omega] \in H^{2}(M, \mathbf{R})$ and the Ricci form of $g^{\prime}$ is $\mathscr{R}^{\prime}$

In particular $c_{1}(T M)=0$ can be represented by $\mathscr{R}^{\prime} \equiv 0$ and then according to the above there exists a unique metric $g^{\prime}$ whose Ricci form is $\mathscr{R}^{\prime}$. Therefore its Ricci tensor is vanishes.

One can formulate simpler equivalent versions of (C.a) and (C.b) as requirements on the existence of functions on $M$ as follows. $\mathscr{R}-\mathscr{R}^{\prime}$ is a $\bar{\partial}$ exact and $d$ closed real $(1,1)$ form. By the $\partial, \bar{\partial}$ Lemma one has a real function $f$ on $M$ so that $\mathscr{R}-\mathscr{R}^{\prime}=i \partial \bar{\partial} f$ up to a constant $\kappa$. Recalling (7.16) how $\mathscr{R}$ is derived from the positive function multiplying $w_{1} \wedge \ldots \wedge w_{2 n}$ in (7.13), which is itself determined by $\frac{\omega^{n}}{n !}$, we conclude that $f$ must make its appearance also in $e^{f} \omega^{n}=\left(\omega^{\prime}\right)^{n}$. In fact the 
constant $\kappa$ can be fixed by normalizing the volume $\int_{M} e^{f} \omega^{m}=\int_{M} \omega^{m}$. The simplification is that instead of requiring $g^{\prime}$ to lead to a prescribed $\mathscr{R}^{\prime}$ one requires that it leads to a prescribed volume form and the statement about $\mathscr{R}$ and $\mathscr{R}^{\prime}$ can be replaced by a statement about $f$. Similarly one can formulate the $\left[\omega^{\prime}\right]=[\omega]$ condition in (C.b) as a search for a real function $\phi$ as in (7.15). $\phi$ can be made unique by requiring $\int_{M} \phi \operatorname{vol}_{g}=0$. So the simplified version of (C.a) and (C.b) is

- (C'.a) that for every given Kähler metric $g$, Kähler form $\omega$ and a real smooth function $f$ on $M$ with $\int_{M} e^{f} \omega^{m}=\int_{M} \omega^{m}$

one can construct

- (C'.b) a unique smooth real function $\phi$ on $M$ such that (i) $\omega+i \partial \bar{\partial} \phi$ is a positive $(1,1)$ form $\omega^{\prime}$, (ii) $\int_{M} \phi \operatorname{vol}_{g}=0$ and (iii) $(\omega+i \partial \bar{\partial} \phi)^{m}=e^{f} \omega^{m}$.

Yau proved that the non-linear p.d.e (iii) on $\phi$ admits a unique solution which fulfill (i) and (ii). This is an existence proof and up to date no explicit solutions for $\phi$ and ${ }^{37}$ e.g. the Ricci-flat metric on any compact Calabi-Yau manifold has been given.

c.) $\rightarrow$ e.) at the end of Sec. 7.4 we argued that the holonomy group of a Kähler manifold is generically $U(n)$. Moreover we saw that the Ricci-tensor is generating the $U(1)$ part of $U(n) \cong$ $S U(n) \times U(1)$. On a Ricci-flat manifold this is not generated and the holonomy is reduced to $S U(n)$.

e.) $\rightarrow$ d.) An $(n, 0)$-form can always be locally written as $\Omega_{i_{1}, \ldots, i_{n}}=f(x) \varepsilon_{i_{1}, \ldots i_{n}}$. It is therefore in the total antisymmetric representation of the holonomy group $S U(n)$ i.e. a singlet invariant under Hol. By the fact quoted in the last paragraph of Sec. 7.4 one has that $\nabla \Omega=0$. Since $\Gamma$ has no mixed indices $\bar{\partial}_{\bar{i}} \Omega=\nabla_{\bar{i}} \Omega=0$ and $\Omega$ is holomorphic. This implies that $f(x)$ has to be a globally defined holomorphic holomorphic function over the compact manifold $M$ and hence a constant. Note that $\omega$, locally written as $\omega=\frac{i}{2}\left(\mathrm{~d} x^{1} \wedge \mathrm{d} x^{\overline{1}} \wedge \ldots \wedge \mathrm{d} x^{n} \wedge \mathrm{d} x^{\bar{n}}\right)$, and $g$, locally written $g=\sum_{i=1}^{n}\left|\mathrm{~d} x^{i}\right|^{2}$, are also covariantly constant. The normalization (7.51) established at a point requires $|f|=1$, but is since all quantities are covariantly constant (7.51) will hold at any point.

$\Omega$ is also harmonic $\Delta_{\bar{\partial}} \Omega=0$ as beside $\bar{\partial} \Omega=0$ also $\bar{\partial}^{*} \Omega=-* \partial * \Omega=0$, because $*: A^{n, 0} \rightarrow$ $A^{n, 0}$ and $\partial: A^{n, 0} \rightarrow A^{n+1,0}=\{0\}$.

d.) $\rightarrow$ a.) We just constructed with $\Omega$ a trivial constant section of the canonical bundle $\wedge^{n} T^{*(1,0)} M$.

d) $\rightarrow$ b): Assume a nowhere vanishing holomorphic $(n, 0)$ exists. We get then a globally well defined scalar function

$$
|\Omega|^{2}=\frac{1}{n !} \Omega_{i_{1} \ldots i_{n}} \bar{\Omega}^{i_{1} \ldots i_{n}},
$$

where the indices are raised by the hermitian metric $g^{i_{k} \bar{j}_{k}}$. Locally $\Omega$ is given by $\Omega_{i_{1}, \ldots, i_{n}}=$ $f(x) \varepsilon_{i_{1}, \ldots i_{n}}$, where $f(x)$ is a non-vanishing holomorphic function in each patch. We can obtain $\bar{\Omega}^{i_{1}, \ldots i_{n}}=\frac{\bar{f}}{g} \varepsilon^{i_{1} \ldots i_{n}}$ and it follows that $g=\operatorname{det}\left(g_{i \bar{j}}\right)=\frac{|f|^{2}}{\|\left.\Omega\right|^{2}}$. Inserting in (7.35) we get $c_{1}(T M)=$ $-\frac{i}{2 \pi} \partial \bar{\partial} \log |\Omega|^{2}$ which is exact since $\log |\Omega|^{2}$ is a scalar, hence $c_{1}(T M)=0$ in cohomology.

f.) $\leftrightarrow$ d.) is proven in generality in [147]. This is done using representation theory. Let us just give a simple relevant example namely the threefold case, $n=3$. We must figure out how many

\footnotetext{
${ }^{37}$ It is not that difficult to find a Kähler metric on a Calabi-Yau, e.g. by constructing the induced metric of the Fubini-Study metric on the quintic in $\mathbf{P}^{4}$, see [140].
} 
spinors transforming as singlets under the holonomy $S U(3)$. Under generic rotations in the internal $6 \mathrm{~d}$ space vectors transform by $S O(6)$ and the associated spin group with the same Lie algebras is isomorphic to $S U(4)$. The spinor representation in $6 d$ is $2^{\frac{6}{2}}=8$ dimensional and splits according to the chirality into representations $(\mathbf{4}, \overline{\mathbf{4}})$ of this $S U(4)$. Now the holonomy is reduced to $S U(3)$ and embedding the $S U(3)$ in $S U(4)$ singles out an $U(1)$, i.e. one has $S U(3) \otimes U(1) \in S U(4)$. The decomposition of the $(\mathbf{4}, \overline{\mathbf{4}})$ into the representations of this $U(1)$ and $S U(3)$ is unique $(\mathbf{4}, \overline{\mathbf{4}})=$ $\left(3^{1} \otimes \mathbf{1}^{-3}, \overline{\mathbf{3}}^{-1} \otimes \mathbf{1}^{3}\right)$, where the superscripts are the $U(1)$-charges. Hence we can conclude that there are indeed one invariant and therefore covariantly constant spinor of each helicity. Bilinear of the covariantly constant spinors can be used to build the covariantly constant tensors discussed above. In particular the almost complex structure is $J_{b}^{a}=-i \xi^{\dagger} \Gamma_{b}^{a} \xi$, the metric $g_{\mu \bar{v}}=i \xi^{\dagger} \Gamma_{\mu, \bar{v}} \xi$ and the $(3,0)$ form by $\Omega_{i j k}=e^{-i \alpha} \xi^{T} \Gamma_{i j k} \xi$. In this way one can show f.) $\rightarrow$ d.) see [21] for details. Furthermore it is easy to see that the eight spinors can be generated from $\xi \in \mathbf{1}^{-3}$ as $\Gamma_{i} \xi \in \overline{\mathbf{3}}^{-1}$, $\Gamma_{i j} \xi \in \mathbf{3}^{1}, \Gamma_{i j k} \xi \in \mathbf{1}^{3}$ and decomposed as

$$
\eta=\Omega^{0,0} \xi+\Omega_{\bar{l}}^{0,1} \Gamma^{\bar{c}} \xi+\Omega_{\bar{l} \bar{j}}^{0,2} \Gamma^{\bar{i} \bar{j}} \xi+\Omega_{\bar{\imath} \bar{j} k}^{0,3} \Gamma^{\bar{i} \bar{j} k} \xi \quad \text { where } \Omega_{\bar{l}_{1} \ldots l_{r}}^{0, n} \mathrm{~d} x^{\bar{l}_{1}} \wedge \mathrm{d}^{\bar{l}_{r}} \in H_{\bar{\partial}}^{0, r}(M) .
$$

On $T_{\mathbf{C}}^{3}$ one has therefore eight covariant constant spinors and on $T_{\mathbf{C}}^{1} \times K 3$ four.

A very general tool in Čheck chomology is Serre duality which states for any sheaf $E$ on $M$ that

$$
H^{k}(E)^{*} \cong H^{n-k}\left(E^{*} \otimes K_{M}\right)
$$

Using Čheck-Dolbeault isomorphism $H^{k}(E) \cong H_{\bar{\partial}}^{k}(M, E), H^{r}\left(M, \wedge^{s} T^{*} M\right)=H^{s, r}(M)$ and $K_{M}=1$ we relate a Calabi-Yau manifold by taking $E=\mathscr{O}(M)$ the cohomology groups $H^{0, r}(M) \cong H^{0, n-r}(M)$ or by complex conjugation the cohomology goups $H^{r, 0}(M) \cong H^{n-r, 0}(M)$. This particular result can be seen also in a more direct way by contracting a $(p, 0)$ form $\omega_{i_{1} \ldots i_{p}} \mathrm{~d} x^{i_{1}} \wedge \ldots \wedge \mathrm{d} x^{i_{p}}$ with the unique $(0, n)$ form $\hat{\omega}_{\bar{j}_{p+1} \ldots \bar{j}_{n}}=\frac{1}{p !} \bar{\Omega}_{\bar{j}_{1} \ldots \bar{j}_{n}} \omega^{\bar{j}_{1} \ldots \bar{j}_{p}}$ to define a $(0, n-p)$-form $\hat{\omega}$. One shows easily that this is an invertible map that commutes with $\Delta$, i.e. $H^{p, 0}(M) \cong H^{0, n-p}(M) \cong H^{n-p, 0}(M)$.

With $h^{n, 0}(M)=h^{0,0}=1$ e.q. (7.53) implies that one has at least two covariantly constant spinors on a Ricci-flat manifold. In order to show that one has only this two on a manifold with Hol $=S U(n)$ we shall show that $h^{p, 0}=0$ for $0<p<n$. On a compact Kähler manifold harmonicity of $(p, 0)$-form implies holomorphicity as argued after (7.24) by consideration of type. Specializing (7.27) to $R_{i j \bar{k} \bar{l}}=0$ for Kähler- and $R_{i \bar{j}}=0$ for Ricci-flat manifolds harmonicity means $\nabla^{\nu} \nabla_{\nu} \omega_{i_{1} \ldots i_{p}}=0$. On a compact manifold one can use pairing and partial integration to see that this requires $\nabla_{j} \omega_{i_{1} \ldots i_{p}}=0$ (and also $\bar{\partial} \omega=0$ ). From these equations we conclude that all harmonic $(p, 0)$ forms are covariantly constant. However that would mean that they are invariant under $S U(n)$, which is impossible for $0<p<n$ as only the trivial and the total antisymmetric representation are invariant.

\subsection{Bergers List}

Let us finally show here Bergers list of the possible holonomy groups and their representation on the tangent space of simply connected irreducible and non-symmetric manifolds of real dimension $m$ with the additional information about the number $N_{+}, N_{-}$of complex covariant constant spinors with positive and negative chirality [147] respectively. If $m$ is odd the spinor representation 
is irreducible and we have just one type of spinor. The last part comments on the special forms that exist on this manifold. See [86] for background.

- (i) generic oriented: $\operatorname{Hol}(g)=S O(m)$, not nec. spin.

- (ii) $n=2 m$ with $m \geq 2$ : $\operatorname{Hol}(g)=U(m)$, not nec. spin, $\omega(1,1)$ Kähler form

- (iii) $m=2 n, n \geq 2: \operatorname{Hol}(g)=S U(n)$, vector, $N_{ \pm}=1$ for $n$ odd, $N_{+}=2$ for $n$ even, $\omega(1,1)$ Kähler form and $\Omega(n, 0)$ holomorphic form

- (iv) $m=4 n, n \geq 2: \operatorname{Hol}(g)=S p(n)$, vector, $N_{+}=m+1, H, I, J S U(2)$ triplet of $(1,1)$ forms.

- (v) $m=7: \operatorname{Hol}(g)=G_{2}, 7$ dim irred., $N=1, \Phi$ associative 3-form, $* \Phi$ coassociative 4-form.

- (vi) $m=8: \operatorname{Hol}(g)=S p(7)$, spin, $N_{-}=1, \Psi$ Cayley 4-form.

\subsection{Examples of Calabi-Yau spaces}

The tool that makes constructing of Calabi-Yau spaces easy is the perfect control over the first Chern class in algebraic geometry. As an application of some statements in Sec. 7.3 we want to calculate the first Chern class of $\mathbf{P}^{n}$, following [16]. As every projective space $\mathbf{P}^{n}$ has a tautological sequence

$$
0 \rightarrow H^{*} \rightarrow \mathbf{P}^{n} \times \mathbf{C}^{n+1} \rightarrow Q \rightarrow 0 .
$$

$H^{*}=\left\{(l, x) \in \mathbf{P}^{n} \times \mathbf{C}^{n+1} \mid x \in \hat{l}\right\}$, where $\hat{l}$ is the line in $\mathbf{C}^{n+1}$, which defines $l$ as point in $\mathbf{P}^{n}$, and the quotient space $Q$ is defined by (7.55). $H^{*}$ is parametrized by the homogeneous variables $\left[x_{1}: \ldots\right.$ : $x_{n+1}$ ], which, as maps to $\mathbf{C}$, are section of the dual space $H$, called the hyperplane bundle. We can write tangent vectors in $T \mathbf{P}^{n}$ as linear combinations of $\left(\sum_{k=1}^{n+1} a_{k}^{i} x_{k}\right) \frac{\partial}{\partial x_{i}}$, which is scaling invariant under the $\mathbf{C}^{*}$ action and maps $H^{\oplus(n+1)}$ to $T \mathbf{P}^{n}$. There is a kernel $\mathbf{C}$ of that map, namely we have $\sum x_{i} \frac{\partial}{\partial x_{i}}=0 \in T \mathbf{P}^{n}$ as it just generates the scaling action. These facts are expressed in the Euler sequence

$$
0 \rightarrow \mathbf{C} \rightarrow H^{\oplus(n+1)} \rightarrow T \mathbf{P}^{n} \rightarrow 0 .
$$

The Chern class of $\mathbf{C}$ is 1 and the Whitney formula and (trivial) splitting principle gives

$$
c\left(T \mathbf{P}^{n}\right)=(1+x)^{n+1},
$$

where we appreciated $x=c_{1}(H)$.

A weighted projective space $W C P^{n}$ is defined similarly as $\mathbf{P}^{n}$ cff. (7.2), only that $\mathbf{C}^{*}$ acts now by

$$
\left(x_{1}, \ldots, x_{n+1}\right) \sim\left(\lambda^{w_{1}} x_{1}, \ldots, \lambda^{w_{n+1}} x_{n+1}\right),
$$

where common factors in all weights $w_{i}$ can be scaled out. Common factors $k$ in subsets of the weights lead to $Z_{k}$ quotient singularities of $W C P^{n}$. A similar argument as before shows that [46.

$$
c\left(T W C P^{n}\right)=\prod_{i=1}^{n+1}\left(1+w_{i} x\right),
$$


All weights are in $\mathbf{Z}$ and order to be compact $w_{i}>0$. This prevents us to define compact $W C P$ with $c_{1}\left(T W C P^{n}\right)=0$, but $W C P(-3,1,1,1)$ is a well known example of a non-compact Calabi-Yau manifold, better know as $\mathscr{O}(-3)$ line bundle over $\mathbf{P}^{2}$.

Compact examples are easily obtained, e.g. as hypersurfaces in the projective spaces above. Let us consider a smooth degree $d$ hypersurface $M$ in $\mathbf{P}^{n}$. M is defined as zero locus of a degree $d$ polynomial $P$, which is sufficiently general so that $P=0$ and $\mathrm{d} P=0$ has no common solution. It is a section of $H^{d}=\mathscr{O}_{\mathbf{P}^{n}}(d)$. Since $P$ is smooth we have a splitting of the tangent bundle $T \mathbf{P}^{n}$ as follows

$$
\left.0 \rightarrow T M \rightarrow T \mathbf{P}^{n}\right|_{M} \rightarrow N_{M} \rightarrow 0,
$$

where $N_{M}$ is the normal bundle to $M$, which is identified with $\left.\mathscr{O}(d)\right|_{M}$ because $P$ is a coordinate of $N$ near $M \cdot \operatorname{Ch}\left(H^{d}\right)=e^{d x}=1+c_{1}\left(H^{d}\right)=1+d x$, i.e. $c_{1}\left(H^{d}\right)=d x$ and the adjunction formula gives

$$
c(M)=\frac{(1+x)^{n+1}}{(1+d x)}=1+(n+1-d) x+\ldots,
$$

i.e. a Calabi-Yau hypersurface in $\mathbf{P}^{n}$ has to have degree $d=n+1$. In this case $P$ is a section $\mathscr{O}\left(K \mathbf{P}^{n}\right)$ of the canonical line bundle $K=-\left[c_{1}\left(\mathbf{P}^{n}\right)\right]$. This gives for dimension three case, the quintic in $\mathbf{P}^{4}$. For weighted projective spaces one has

$$
c(M)=\frac{\prod_{i=1}^{n+1}\left(1+w_{i} x\right)}{(1+d x)}=1-\left(d-\sum_{i} w_{i}\right) x+\ldots
$$

Together with the transversality condition $\mathrm{d} P=0$ at $P=0$ it leads 7555 examples of Calabi-Yau threefolds [96]. This sample contains many mirror pairs.

Batyrev provided a systematic construction of mirror pairs, as sections $M=\mathscr{O}\left(K_{\left(\mathbf{P}_{\Delta}\right)}\right)$ and $W=$ $\mathscr{O}\left(K_{\mathbf{P}(\Delta *)}\right)$ respectively[8]. Here $\mathbf{P}_{\Delta}$ is the projective space associated to the integral polyhedron $\Delta$ [59]. Batyrev showed that if the $\Delta$ polyhedron is reflexive then a smooth sections of $\mathscr{O}\left(K_{\mathbf{P}(\Delta)}\right)$ exists, the dual reflexive polyhedron $\Delta^{*}$ exists and the generically smooth section of $\mathscr{O}\left(K_{\mathbf{P}(\Delta)}\right)$ has mirror Hodge numbers $h^{p, q}(M)=h^{3-p, q}(W)$. Reflexive polyhedra in four dimensions relevant for the CY threefold case have been classified [102]. These and generalized constructions like complete intersections and orbifolds of tori and the afore mentioned manifolds are the bulk of the systematically explored examples of Calabi-Yau mirror pairs, see [103] for computer generated lists with about $10^{4}-10^{8}$ topological inequivalent examples ${ }^{38}$, though slightly more exotic cases, e.g. hypersurfaces and complete intersections in Grassmannians and flag manifolds do exist in unknown numbers. An encouraging observation in view of this enormous numbers is that at least in Type II string theory there is in some sense only one connected component of the Calabi-Yau moduli space. In fact a conjecture formulated by Miles Reid that all Calabi-Yau spaces are in the same moduli space connected by singular transitions [129] finds a physical application in that [134] shows that the singularity in physical quantities as calculated in conformal field theory at the conifold transition between topological different Calabi-Yau spaces is merely a breakdown of the perturbative low energy description due to a non-perturbative black hole becoming massless at the transition point. The full non-perturbative theory at low energy exhibits spontaneous breaking by

\footnotetext{
${ }^{38}$ The lower number is the number of inequivalent Hodge numbers the higher is an estimate of all topological different phases in the Kählercone, which have not been systematically constructed.
} 
acquiring an Higgs vacuum expectation value. Also it has been shown that all hypersurfaces in toric Calabi-Yau can be connected by such physically innocuous transitions.

Acknowledgement: It is a pleasure to thank Mina Aganagic, Robbert Dijkgraaf, Marcos Mariño, Sheldon Katz, Stefan Theisen and Cumrun Vafa for collaboration on the topics presented in this lecture. I want to thank Ian Ellwood, Aki Hashimoto, Minxin Huang, Melissa Liu, Marcos Mariño, Andrew Neitzke, Vasily Pestun, Ingo Runkel and Stefan Theisen and the students of 831 for literature hints and discussions, which helped in preparing this notes.

\section{References}

[1] M. Aganagic, A. Klemm, M. Marino and C. Vafa, The topological vertex, arXiv:hep-th/0305132.

[2] M. Aganagic, R. Dijkgraaf, A. Klemm, M. Marino and C. Vafa, Topological strings and integrable hierarchies, arXiv:hep-th/0312085.

[3] L. Alvarez-Gaume, C. Gomez, G. W. Moore and C. Vafa, Strings In The Operator Formalism, Nucl. Phys. B 303, 455 (1988).

[4] I. Antoniadis, E. Gava, K. S. Narain and T. R. Taylor, N=2 type II heterotic duality and higher derivative F terms, Nucl. Phys. B 455 (1995) 109 [arXiv:hep-th/9507115].

[5] P. S. Aspinwall, B. R. Greene and D. R. Morrison, J. Math. Phys. 35 (1994) 5321.

[6] M. F. Atiyah and R. Bott, The moment map and equivariant cohomology, Topology 23 (1984) 1 .

[7] N. Barth, C. Peters, W. van de Ven "Compact Complex Surfaces," Springer Berlin (1984).

[8] V. Batyrev,Dual Polyhedra and Mirror Symmetry for Calabi-Yau Hypersurfaces in Toric Varieties, J. Algebraic Geom. 3 (1994), no. 3, 493-535

[9] S. Barannikov and M. Kontsevich, Frobenius Manifolds and Formality of Lie Algebras of Polyvector Fields, Internat. Math. Res. Notices 4 (1998) 201 - 215 [alg-geom/9710032].

[10] M. Bershadsky, S. Cecotti, H. Ooguri and C. Vafa, Holomorphic anomalies in topological field theories, Nucl. Phys. B 405 (1993) 279 [arXiv:hep-th/9302103].

[11] M. Bershadsky, S. Cecotti, H. Ooguri and C. Vafa, Kodaira-Spencer theory of gravity and exact results for quantum string amplitudes, Commun. Math. Phys. 165 (1994) 311 [arXiv:hep-th/9309140].

[12] A. L. Besse, Einstin Manifolds, Springer, Berlin (1987).

[13] D. Bessis, C. Itzykson and J. B. Zuber, Quantum Field Theory Techniques In Graphical Enumeration, Adv. Appl. Math. 1, 109 (1980).

[14] J.-M. Bismut, H. Gillet and C. Soulé, Analystic Torsion and Holomorphic Determinant Line Bundles III, Quillen Metrics on Holomorphic Determinants, Comm. Math. Phys. 115 (1998) 49.

[15] R. Borcherds, Automorphic forms with singularities on Grassmannians, alg-geom/9609022.

[16] R. Bott and L.W. Tu Differential forms in algebraic Topology, Springer Berlin (1982).

[17] G.E. Bredon, Introduction to compact transformation Groups, Academic Press (1972) New York .

[18] I. Brunner, M. R. Douglas, A. E. Lawrence and C. Romelsberger, JHEP 0008, 015 (2000) [arXiv:hep-th/9906200]. 
[19] R. Bryant and P. Griffith, Some observations on the Infinitessimal Period Relations for Regular Threefolds with Trivial Canonical Bundle, in Prgress in Mathematics 36, M. Artin and J. Tate, Eds.Birkhäuser, (1983).

[20] A. Capelli, C. Itzykson, and J.B. Zuber, Modular Invariant Partition functions in Two Dimensions, Nucl. Phys. B280 [FS 18] (1987) 445, A. Capelli, Modular Invariant Partition Functions of Superconformal Theories, Phys. Lett. B215 (1987) 82.

[21] P. Candelas and X. del la Ossa, Lectures on Complex Manifolds, in Superstrings '87, "Proceedings of the Trieste Spring School,” Ed. L. Alvarez-Gaume et. al., World Scientific, Singapore (1987).

[22] P. Candelas and X. de la Ossa, Moduli Space Of Calabi-Yau Manifolds, Nucl. Phys. B 355, 455 (1991).

[23] P. Candelas, P. S. Green and T. Hubsch, Rolling Among Calabi-Yau Vacua,” Nucl. Phys. B 330, 49 (1990).

[24] P. Candelas, G. Horowitz, A. Strominger and E. Witten, Vaccum Configurations for Superstrings, Nucl. Phys. B256 (1985) 46.

[25] P. Candelas, X. C. De La Ossa, P. S. Green and L. Parkes, A Pair Of Calabi-Yau Manifolds As An Exactly Soluble Superconformal Theory, Nucl. Phys. B 359, (1991) 21.

[26] P. Candelas, X. De La Ossa, A. Font, S. Katz and D. R. Morrison, Mirror symmetry for two parameter models. I, Nucl. Phys. B 416 (1994) 481 [arXiv:hep-th/9308083].

[27] P. Candelas and A. Font, Duality between the webs of heterotic and type II vacua, Nucl. Phys. B 511 (1998) 295 [arXiv:hep-th/9603170].

[28] S. Cecotti, S. Ferrara and L. Giradello, Goemetry of Type II superstrings and the moduli of superconformal field theories, Int. J. Mod. Phys. A4 (1989) 2475.

[29] S. Cecotti Nucl. Phys. B 355 (1991) 755.

[30] S. Cecotti and C. Vafa, Ising model and N=2 supersymmetric theories, Commun. Math. Phys. 157, 139 (1993) [arXiv:hep-th/9209085].

[31] S. Cecotti and C. Vafa, Topological Anti-topological Fusion, Nucl. Phys. B 367 (1991) 369.

[32] D. A. Cox and S. Katz, Mirror Symmetry And Algebraic Geometry, Mathematical Surveys and Monographs, 68 (1999), American Mathematical Society, Providence, RI

[33] D. Cox, The Homogeneous coordinate ring of a toric variety, J. Alg. Geom. 4 (1995) 17 [alg-geom/9210008].

[34] B. Craps, F. Roose, W. Troost and A. Van Proyen, Nucl. Phys. B503, (1997) 565, hep-th/9703082.

[35] E. Cremmer et. al, Vector Multiplets Coupled to N=2 Supergravity: Superhiggs Effect, Flat Potentials and Goemtric Structures, Nucl. Phys. 250 (1985) 385.

[36] V. I .Danilov, The geometry of toric varieties, Russian Math. Survey 33, n.2 (1978) 97

[37] B. de Wit and A. van Proyen, Potentials and symmetries of General Gauged N=2 Supergravity-Yang-Mills Models, Nucl. Phys. 245 (1984) 89

[38] B. de Wit, P.G. Lauwers and A. Van Proyen, Lagrangians of N=2 Supergravity Matter Systems, Nucl. Phys. B255 (1980) 569, 
[39] P. Di Francesco, P. H. Ginsparg and J. Zinn-Justin, 2-D Gravity and random matrices, Phys. Rept. 254, 1 (1995)

[arXiv:hep-th/9306153].

[40] R. Dijkgraaf, Intersection theory, integrable hierarchies and topological field theory, arXiv:hep-th/9201003.

[41] R. Dijkgraaf, E. Verlinde and H. Verlinde Loop Equations and the Virasoro constraints in non-perturbative quantum gravity, Nucl. Phys. B 384 (1991) 435.

[42] R. Dijkgraaf, H. Verlinde and E. Verlinde, Topological strings in d<1, Nucl. Phys. B 352 (1991) 59.

[43] Tammo tom Dieck, Transformation Groups, Walter de Gruyter Berlin (1987).

[44] R. Dijkgraaf, Fields, strings, matrices and symmetric products, arXiv:hep-th/9912104.

[45] L. J. Dixon Some world-sheet properties of superstring compactifications, on orbifolds and otherwise, In "Superstrings, Unified Theories, and Cosmology," (1987) Ed. G. Furla et al., World Scientific, Singapore.

[46] V. Dolgachev, Weighted projective spaces, Lectures Notes in Mathematics, Springer .

[47] S. K. Donaldson, R. P. Thomas, Gauge theory in higher dimensions, in The geometric universe, 31-47, (1998) Oxford Univ. Press.

[48] T Eguchi, P.B. Gilkey and A. Hanson, Gravitation, Gauge Theory and Differential Geometry, Phys. Rep. 66 (1980) 213.

[49] T. Eguchi and S.-K. Yang, N=2 superconformal models as topological field theories, Mod. Phys. Lett. A5 1693 (1990).

[50] J. Erler and A. Klemm, Comment on the generation number in orbifold compactifications, Commun. Math. Phys. 153, 579 (1993) [arXiv:hep-th/9207111].

[51] C. Faber and R. Pandharipande, Hodge integrals and Gromow-Witten theory, Invent. Math 139173 , math.AG./9810173.

[52] C. Faber, Alogarithms for computing intersection numbers on the moduli space of curves, with a application to the locus of the Jacobian, math.AG/9711218.

[53] S. Ferrara, R. Kallosh and A. Strominger, Phys. Rev. D52 (1995) 5412, hep-th/9508072.

[54] A. Font and S. Theisen, Introduction to String Compactification, Lectures (2003) available with excercises at http://www. aei.mpg.de/ theisen.

[55] D. Friedan, “Introduction to Polykov's String Theory,” Les Houches (1882)

[56] J. Fuchs, A. Klemm, C. Scheich and M. G. Schmidt, Spectra And Symmetries Of Gepner Models Compared To Calabi-Yau Compactifications, Annals Phys. 204, 1 (1990).

[57] K. Fujikawa and H. Suzuki, Path integrals and quantum anomalies, Clarendon Press, Oxford (2004).

[58] W. Fulton, Intersection theory, Springer, Berlin (1998).

[59] W. Fulton, Introduction to toric varieties (Princeton Univ. Press, Princeton 1993)

[60] D. Gaiotto and L. Rastelli, A paradigm of open/closed duality: Liouville D-branes and the Kontsevich model, arXiv:hep-th/0312196.

[61] D. Gepner, Spacetime supersymmetries in compactified string theory and superconformal models, Nucl. Phys. B 296, 757. 
[62] D. Gepner, Lectures On N=2 String Theory, Lectures at Spring School on Superstrings, Trieste, Italy, Apr 3-14, 1989

[63] P. H. Ginsparg and G. W. Moore, "Lectures on 2-D gravity and 2-D string theory," arXiv:hep-th/9304011.

[64] D. Ghoshal and C. Vafa, $C=1$ string as the topological theory of the conifold," Nucl. Phys. B 453, 121 (1995) [arXiv:hep-th/9506122].

[65] R. Gopakumar and C. Vafa, M-theory and topological strings. I, [arXiv:hep-th/9809187].

[66] R. Gopakumar and C. Vafa, M-theory and topological strings. II, [arXiv:hep-th/9812127].

[67] M. B. Green, J.H. Schwarz and E. Witten, Superstring Theory, I \& II, Cambridge University Press (1987)

[68] M. Green and N. Seiberg, Nucl. Phys. B299 (1988) 559.

[69] B. R. Greene and M. R. Plesser, Duality In Calabi-Yau Moduli Space, Nucl. Phys. B 338, 15 (1990).

[70] P. Griffith, On the periods of certain rational integrals I and II, Ann. of Math. 90460 and 498 (1969).

[71] P. Griffith, Periods of integrals on algebraic manifolds I and II, Amer. J. Math 90568 and 805 (1970).

[72] P. Griffith and J. Harris, Principles of Algebraic Geometry, J. Wiley \& Sons New York (1978).

[73] P. Griffith (Ed.) Topopics in Transcendental Algebraic Geometry, "Annals of Mathematical Studies," Princeton University Press (1984).

[74] M. T. Grisaru, A. E. M. van de Ven and D. Zanon, Nucl. Phys. B 277, 409 (1986).

[75] D. J. Gross and I. R. Klebanov, One-Dimensional String Theory On A Circle, Nucl. Phys. B 344, 475 (1990).

[76] K. Hori, S. Katz, A. Klemm, R. Pandharipande, R. Thomas, C. Vafa, R. Vakil and E. Zaslow, Mirror Symmetry, American Mathemtical Society (2003).

[77] J. Harris and I. Morrison, Moduli of curves, Vol. 187 of Graduate Texts in Mathematics, Springer-Verlag, Berlin 1998.

[78] A. Hashimoto, M. x. Huang, A. Klemm and D. Shih, Open / closed string duality for topological gravity with matter, arXiv:hep-th/0501141.

[79] S. Hosono, A. Klemm, S. Theisen and S. T. Yau, Mirror symmetry, mirror map and applications to Calabi-Yau hypersurfaces, Commun. Math. Phys. 167 (1995) 301 [arXiv:hep-th/9308122].

[80] S. Hosono, A. Klemm, S. Theisen and S. T. Yau, Mirror symmetry, mirror map and applications to complete intersection Calabi-Yau spaces, Nucl. Phys. B 433 (1995) 501 [arXiv:hep-th/9406055].

[81] F. Hirzebruch, Topological Methods in Algebraic Topology, Springer-Verlg, Berlin (1995) 3rd ed.

[82] G. Horowitz, What is a Calabi-Yau space?, in Proceedings of the Workshop on Unified String theories, Santa Barbara 1985, Eds. M. Green and D. Gross.

[83] P. S. Howe, G. Papadopoulos and K. S. Stelle, Phys. Lett. B 174, 405 (1986).

[84] N. Hitchin, The Geometry of three-forms in six and seven dimensions, arXiv:math.DG/0010054 .

[85] N. Hitchin, Generalized Calabi-Yau manifolds, Q.J. Math. 54 (2003) 281.

[86] D. D. Joyce, Compact Manifolds with special Holonomy, Oxford University Press (2000). 
[87] D. D. Joyce, Compact Riemannian J-manofolds with holonomy $G_{2}$ I II , J. Diff Geometry, 43 (1996) 291,329 .

[88] S. Kachru, A. Klemm, W. Lerche, P. Mayr and C. Vafa, Nucl. Phys. B 459, 537 (1996) [arXiv:hep-th/9508155].

[89] V. Kaplunovsky and J. Louis, On Gauge couplings in string theory, Nucl. Phys. B 444, 191 (1995) [arXiv:hep-th/9502077].

[90] S. Katz, A. Klemm and C. Vafa, M-theory, topological strings and spinning black holes, Adv. Theor. Math. Phys. 3 (1999) 1445 [arXiv:hep-th/9910181].

[91] T. Kawai and K. Yoshioka, String Partition function and Infinite Products [arXiv:hep-th/0002169].

[92] Y. Kazama and H. Suzuki, Characterization Of N=2 Superconformal Models Generated By Coset Space Method, Phys. Lett. B 216, 112 (1989) and New N=2 Superconformal Field Theories And Superstring Compactification, Nucl. Phys. B 321, 232 (1989).

[93] Phys. Usp. 37, 1 (1994) [arXiv:hep-th/9303139].

[94] A. Klemm, arXiv:hep-th/9705131.

[95] A. Klemm and E. Zaslow, Local mirror symmetry at higher genus, in Winter School on Mirror Symmetry, Vector Bundles and Lagrangian Submanifolds (Cambridge, MA, 1999), AMS/IP Stud. Adv. Math. 23 (2001) 183 - 207 [arXiv:hep-th/9906046].

[96] A. Klemm and R. Schimmrigk, Landau-Ginzburg string vacua, Nucl. Phys. B 411, 559 (1994) [arXiv:hep-th/9204060], M. Kreuzer and H. Skarke, On the classification of quasihomogeneous functions, Commun. Math. Phys. 150, 137 (1992) [arXiv:hep-th/9202039].

[97] A. Klemm, M. Kreuzer, E. Riegler and E. Scheidegger, Topological String Amplitudes, Complete intersection Calabi-Yau Spaces and Threshold Corrections, hep-th/0410018.

[98] K. Kodaira, Complex manifolds and Deformations of Complex Structures, Springer-Verlag, Berlin (1985) .

[99] M. Kontsevich, Intersection theory on the moduli space of curves and the matrix Airy function, Commun. Math. Phys. 147, 1 (1992).

[100] M. Kontsevich, "Enumeration of rational curves via Torus actions," arXiv:hep-th/9405035.

[101] M. Kontsevich, Product formulas for modular forms on $O(2, n)$ (after R.Borcherds) [alg-geom/9709006].

[102] M. Kreuzer, H. Skarke, Complete classification of reflexive polyhedra in four dimensions, Adv. Theor. Math. Phys. 4 (2000) 6 [hep-th/0002240]

[103] http://hep.itp.tuwien.ac.at/ kreuzer/CY/hep-th/yymmnnn.html

[104] D. Kutasov, Phys. Lett. B 220 (1989) 153.

[105] J.M.F. Labastida, P.M. Llatas, Topological Matter in Two Dimensions, Nucl.Phys. B379 (1992) 220, arXiv/hep-th/9112051.

[106] J.M.F. Labastida, M. Marino, Type B topological matter, Kodaira Spencer Theory and mirror symmetry, Phys.Lett. B333 (1994) 386, arXiv/hep-th/9405151.

[107] A. Landmann, On the Picard-Lefshetz transformations, Trans. A.M.S. 181 (1973) 89. 
[108] W. Lerche, C. Vafa and N.P. Warner, Chiral Rings in N=2 superconformal Theories, Nucl. Phys. B324 (1989) 371.

[109] A. S. Losev, A. Marshakov and N. A. Nekrasov, "Small instantons, little strings and free fermions," arXiv:hep-th/0302191.

[110] J. Maldacena, G. W. Moore, N. Seiberg and D. Shih, JHEP 0410, 020 (2004) [arXiv:hep-th/0408039].

[111] Y. I. Manin, Frobenius Manifolds, Quantum Cohomology, and Moduli Spaces, Colloquium Publications AMS Vol 47, (1999).

[112] D. Maulik, N. Nekrasov, A. Okounkov and R. Pandharipande, Gromow-Witten theory and Donaldson-Thomas theory I, [arXiv:math.AG/0312059].

[113] M. Marino, Les Houches lectures on matrix models and topological strings, arXiv:hep-th/0410165.

[114] M. Marino, Chern-Simons theory and topological strings, arXiv:hep-th/0406005.

[115] , M. Mirzakhani, Weil-Peterson volumes and the Witten-Kontsevich formula, Preprint (2003), Homepage M. Mirzakhani.

[116] D. R. Morrison, Where is the large radius limit?, arXiv:hep-th/9311049. .

[117] A. Neitzke and C. Vafa, Topological strings and their physical applications, arXiv:hep-th/0410178.

[118] D. Nemeschansky and A. Sen, "Conformal Invariance Of Supersymmetric Sigma Models On Calabi-Yau Manifolds," Phys. Lett. B 178, 365 (1986).

[119] N. Nekrasov, H. Ooguri and C. Vafa, S-duality and topological strings, arXiv:hep-th/0403167.

[120] A. Newlander and L. Nirenberg, Complex analytic coordiantes in almost complex manifolds, Ann. Math. 65 (1957) 351-404

[121] T. Oda, Convex bodies and algebraic geometry (Springer, Berlin Heidelberg 1988).

[122] A. Okounkov and R. Pandharipande Gromow-Witten theory, Hurwitz numbers and Matrix models I, math/AG/0101147.

[123] H. Ooguri and C. Vafa, Knot invariants and topological strings, Nucl. Phys. B 577, 419 (2000) [arXiv:hep-th/9912123].

[124] M. Peskin and D. Schroeder, An Introduction to Quantum field theory, Westview Press (1995).

[125] V. Pestun and E. Witten, The Hitchin functionals and the topological B-model at one loop, arXiv:hep-th/0503083.

[126] J. Polchinski, String Theory, I \& II, Cambridge University Press (1998).

[127] J. Polchinski and A. Strominger, New Vacua for Type II String Theory, Phys. Lett. B 388, 736 (1996) [arXiv:hep-th/9510227].

[128] A. Recknagel and V. Schomerus, Nucl. Phys. B 531, 185 (1998) [arXiv:hep-th/9712186].

[129] M. Reid, The moduli space of 3-folds with $K=0$ may nevertheless be irreducible, Math. Ann. 278 (1987) 329.

[130] A. Schwimmer and N. Seiberg, Comments on the N=2,3,4 superconformal Algebras in two dimensions, Phys. Lett. 184B (1987) 191.

[131] W. Schmidt, Variation of Hodge structure: The singularities of the period map, Inventiones Math. 22 (1973) 211. 
[132] K. Strebel, Quadratic Differentials, Springer 1984.

[133] A. Strominger and E. Witten, Comm. Math. Phys. 101 (1985) 341.

[134] A. Strominger Massless black holes and conifolds in string theory, Nuc. Phys. B245 96.

[135] A. Strominger, Special Geometry, Comm. Math. Phys. 133 (1990) 163.

[136] A. Strominger, Macroscopic Entropy of N=2 Extremal Black Holes, Phys. Lett. B238 (1996) 39, hep-th/9602111.

[137] A. Strominger, Superstrings With Torsion, Nucl. Phys. B 274, 253 (1986).

[138] R. P. Thomas, A holomorphic Casson invariant for Calabi-Yau 3-folds, and bundles on K3 fibrations, J. Differ. Geom. 54 (2000) 367-438, [arXiv:math.ag/9806111].

[139] G. Tian, Smoothness of the Universal Deformation Space of Compact Calabi-Yau manifolds and its Weil-Peterson metric, in "Mathematical Aspects of String Theory" Ed. S.T. Yau, World Scientific Singapore (1987).

[140] G. Tian, Canonical Metrics in Kähler Geoemtry, Birkhäuser (2002).

[141] G. 't Hooft, Perturbative Quantum Gravity, in Proceedings of the International School of Subnuclear Physics, Erice 2002, From Quarks and Gluons to Quantum Gravity, Subnuclear Series Vol. 40, ed. A. Zichichi, World Scientific, 249, http://www.phys.uu.nl/ thooft/

[142] A. Todorov, The Weyl-Peterson Geometry of the Moduli-Space of $S U(n \geq 3)$ (Calabi-Yau) Manifolds I, Commun. Math. Phys. 126 (1989) 325.

[143] C. Vafa, Topological Landau-Ginzburg models, Mod. Phys. Lett. A 6 (1991) 337.

[144] C. Vafa, Topological Landau Ginzburg models, Mod. Phys. Lett. A6 (1991) 337.

[145] E. Verlinde and H. Verlinde, A solution of two-Dimensional Topological Quantum Gravity, Nucl. Phys. B348 (1991) 457.

[146] M. Vonk, A mini-course on topological strings, arXiv:hep-th/0504147.

[147] McKenzie Y. Wang, Parallel Spinors and Parallel Forms, Ann. Global Anal. Geom. 7 (1989) 59-68.

[148] E. Witten, Chern-Simons gauge theory as a string theory, Prog. Math. 133, 637 (1995) [arXiv:hep-th/9207094].

[149] E. Witten, Two-Dimensional Gravity and intersection theory on moduli spaces, Surveys in Diff. Geometry 1 (1991) 243.

[150] E. Witten, Quantum Field theory and the Jones Polynomial, Commun. Math. Phys. 121, 351 (1989) [arXiv:hep-th/9207094].

[151] E. Witten, On the Structure of the Topological Phase of Two-Dimensional Gravity, Nucl. Phys. B 340, 281 (1990).

[152] E. Witten, Two-Dimensional Gravity And Intersection Theory On Moduli Space, Surveys Diff. Geom. 1, 243 (1991).

[153] E. Witten, Algebraic geometry associated with matrix models of two dimensional gravity, Topological Methods in Modern Mathematics, (Stony Brook, NY, 1991), 235-269, Publish or Perish, Houston, TX, 1993.

[154] E. Witten, The N matrix model and gauged WZW models, Nucl. Phys. B 371, 191 (1992). 
[155] E. Witten, Topological Sigma Model, Commun. Math. Phys. 118 (1988) 411.

[156] E. Witten, Mirror Manifolds and Topological Field Theory, arXiv:hep-th/9112056 .

[157] E. Witten, Constraints on supersymmetry breaking, Nucl. Phys. B202 (1982) 253.

[158] E. Witten, Elliptic Genera and Quantum Field Theory, Commun. Math. Phys. 109 (1987) 525.

[159] E. Witten, The index of the Dirac operator in Loop-space, in "Elliptic Curves and Modular Forms in Algebraic Topology”, P.S. Landweber Ed., Lecture Notes in Mathematics 1326, Springer Berlin (1980).

[160] E. Witten, On the Landau description of $N=2$ minimal models, Int. Mod. Phys. A9 (1994) 4783, hep-th/9304026.

[161] E. Witten, Two-Dimensional Models With $(0,2)$ Supersymmetry: Perturbative Aspects, hep-th/0504078.

[162] E. Witten, Phases of N=2 theories in two dimensions, Nucl. Phys. B403 (1993) 159 [hep-th/9301042]

[163] S.-T. Yau, On the Ricci curvature of a compact Kähler manifold and the complex Monge-Ampére equations I, Comm. on pure and applied math.31 (1978) 339.

[164] B. Zumino, Supersymmetry And Kähler Manifolds, Phys. Lett. B 87, 203 (1979). 